\title{
A Theory of Ordinary $p$-Adic Curves
}

\author{
By
}

Shinichi MochIZUKI *

\section{Table of Contents}

\section{Table of Contents}

\section{Introduction}

\section{§0. Statement of Main Results}

\section{\$1. Review of the Complex Theory}

Beltrami Differentials

The Beltrami Equation

The Series Expansion of a Quasiconformal Function

Uniformization of Hyperbolic Riemann Surfaces

Uniformization of Moduli Stacks of Hyperbolic Riemann Surfaces

Quasidisks and the Bers Embedding

The Infinitesimal Form of the Modular Uniformizations

Coordinates of Degeneration

The Parabolic Case

Real Curves

\section{§2. Translation into the Adic Case}

Gunning's Theory of Indigenous Bundles

The Canonical Coordinates Associated to a Kähler Metric

The Weil-Petersson Metric from the Point of View of Indigenous Bundles The Philosophy of Kähler Metrics as Frobenius Liftings

The Dictionary

Received September 8. 1995. Revised October 2, 1996

1991 Mathematics Subject Classification 14H10, 14F30

* Research Instıtute for Mathematical Scıences, Kyoto Unıversity, Kyoto 606, Japan 
Loose Ends

Chapter I: Crystalline Projective Structures

\$0. Introduction

\$1. Schwarz Structures

Notation and Basic Definitions

First Properties of Schwarz Structures

Crystalline Schwarz Structures and Monodromy

Correspondence with $\mathbb{P}^{1}$-bundles

Schwarz Structures and Square Differentials

Normalized $\mathbb{P}^{1}$-bundles with Connection

The Schwarzian Derivative

§2. Inndigenous $\mathbb{B}$ umdlles

Basic Definitions and Examples

First Properties

Existence and de Rham Cohomology

Indigenous Bundles of Restrictable Type

§3. The Obstruction to Global Introinsicity

Introduction of Cohomology Classes

Computation of the Second Chern Class

The Case of Dimension One

Appendix: Relation to the Complex Anallytic Case

Chapter II: Indigenous Bundles in Characteristic $\mathbb{P}$

§o. Introduction

§1. $\quad F L$ - B Bundles

Deformations and FL-Bundles

The $p$-Curvature of an FL-Bundle

\$2. The Verschiebung on Indigenous Bundles

The Definition of the Verschiebung

The $p$-Curvature of an Admissible Indigenous Bundle

The Infinitesimal Verschiebung 
Differential Criterion for Admissibility

\section{§3. Hyperbolically Ordinary Curves}

Basic Definitions

The Totally Degenerate Case

The Case of Elliptic Curves: The Parabolic Picture

The Case of Elliptic Curves: The Hyperbolic Picture

The Generic Uniformization Number

\section{Chapter III: Canonical Modular Frobenius Liftings}

\section{\$0. Introduction}

\section{§1. Generalities on Ordinary Frobenius Liftings}

Basic Definitions

The Uniformizing Galois Representation

The Canonical $p$-Divisible Group

Logarithms of Periods

Compatibility of Differentials

Canonical Liftings of Points in Characteristic $p$

Canonical Multiplicative Parameters

Canonical Affine Coordinates

The Relationship between Affine and Multiplicative Parameters

\section{\$2. Construction of the Canonical Frobenius Lifting}

Modular Frobenius Liftings

Indigenous Sections of $\mathscr{D}$

Frobenius Invariant Indigenous Bundles

\section{§3. Applications of the Canonical Frobenius Liftings}

Canonical Liftings of Curves over Witt Vectors

Canonical Affine Coordinates on $\boldsymbol{M}_{g, r}$

Topological Markings and Uniformization by Quadratic Differentials

Canonical Multiplicative Parameters

The Case of Elliptic Curves

\section{Chapter IV: Canonical Curves}

\section{§.. Introduction}


\$1. The Camonical Galois $\mathbb{R}$ epresentation

Construction and Global Properties

The Horizontal Section over the Ordinary Locus

The Canonical Frobenius Lifting over the Ordinary Locus

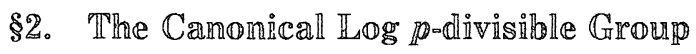

$\log p$-divisible Groups at Infinity

Construction of the Canonical $\log p$-Divisible Group

Review of the Theory of [Katz-Mazur]

§3. The Compactified Camonical Frobenius Lifting

The Canonical Frobenius Lifting and the Canonical Log $p$-Divisible Group

Local Analysis at Supersingular Points

Global Hecke Correspondences

\$4. pp-adic Green's Funnetions

Compactified Frobenius Liftings

The Height of a Frobenius Lifting

Admissible Frobenius Liftings

Geometric Criterion for Canonicality

Chapter V: Uniformizations of Oredinary Curves

\$0. Introduction

\$1. Crystalline Inducution

The Crystalline-Induced $M \mathscr{F}^{\nabla}$-object

The Ring of Additive Periods

The Crystalline-Induced Galois Representation

Relation to the Canonical Affine Coordinates

The Parabolic Case

§2. Camonical Objects over the Stack of Multiplicative $\mathbb{P e r i o d s}$

The Stack of Multiplicative Periods

The Canonical Log $p$-Divisible Group

The Canonical Frobenius Lifting

Bithliography

Indlex 


\section{Introduction}

\section{§o. Statement of Main Results}

The goal of this paper is to present a theory of $r$-pointed stable curves of genus $g$ over $p$-adic schemes (for $p$ odd), which, on the one hand, generalizes the Serre-Tate theory of ordinary elliptic curves to the hyperbolic case (i.e., $2 g$ $-2+r \geq 1$ ), and, on the other hand, generalizes the complex uniformization theory of hyperbolic Riemann surfaces (reviewed in $\S 1$ of this introductory Chapter) due to Ahlfors, Bers, et al. to the $p$-adic case. We begin by setting up the necessary algebraic machinery: that is, the language of indigenous bundles (due to Gunning, although we rephrase Gunning's results in a more algebraic form). An indigenous bundle is a $\mathbb{P}^{1}$-bundle over a curve, together with a connection, that satisfy certain properties. One may think of an indigenous bundle as an algebraic way of encoding uniformization data for a curve. We then study the $p$-curvature of indigenous bundles in characteristic $p$, and show that a generic $r$-pointed stable curve of genus $g$ has a finite, nonzero number of distinguished indigenous bundles $\left(P, \nabla_{P}\right)$, which are characterized by the following two properties:

(1) the $p^{-c u r v a t u r e ~ o f ~}\left(P, \nabla_{P}\right)$ is nilpotent;

(2) the space of indigenous bundles with nilpotent $p$-curvature is étale over the moduli stack of curves at $\left(P, \nabla_{P}\right)$.

We call such $\left(P, \nabla_{P}\right)$ nilpotent and ordinary, and we call curves ordinary if they admit at least one such nilpotent. ordinary indigenous bundle. If a curve is ordinary, then choosing any one of the finite number of nilpotent, ordinary in digenous bundles on the curve completely determines the "uniformization theory of the curve" - to be described in the following paragraphs. Because of this, we refer to this choice as the choice of a p-adic quasiconformal equivalence class to which the curve belongs.

After studying various basic properties of ordinary curves and ordinary indigenous bundles in characteristic $p$. we then consider the $p$-adic theory. Let $\bar{M}_{g, r}$ be the moduli stack of $r^{-}$pointed stable curves of genus $g$ over $\mathbb{Z}_{p}$. Then we show that there exists a canonical p-adic (nonempty) formal stack $\overline{\mathcal{N}}_{g, r}^{\text {ord }}$ together with an étale morphism 


$$
\overline{\mathcal{N}}_{g, r}^{\text {ord }} \rightarrow \bar{M}_{g, r}
$$

such that modulo $p, \overline{\mathcal{N}}_{g, r}^{\text {ord }}$ is the moduli stack of ordinary $r$-pointed curves of genus $g$, together with a choice of $p$-adic quasiconformal equivalence class. Moreover, the generic degree of $\overline{\mathcal{N}}_{g, r}^{\text {ord }}$ over $\bar{M}_{g, r}$ is $>1$ (as long as $2 g-2+r \geq 1$ and $p$ is sufficiently large). It is over $\overline{\mathcal{N}}_{g, r}^{\text {ord }}$ that most of our theory will take place. Our first main result is the following:

Theorem 0.1. Let $\mathscr{C}^{\log } \rightarrow\left(\overline{\mathcal{N}}_{g, r}^{\text {ord }}\right)^{\log }$ (where the "log" refers to canonical $\log$ stack structures) be the tautological ordinary $r$-pointed stable curve of genus $g$. Then there exists a canonical Frobenius lifting $\Phi_{\mathcal{N}}^{\log }$ on $\left(\overline{\mathcal{N}}_{g, r}^{\text {ord }}\right)^{\log }$, together with a canonical indigenous bundle $\left(P, \nabla_{P}\right)$ on $\mathscr{C}^{\log }$. Moreover, $\Phi_{N}^{\log }$ and $\left(P, \nabla_{P}\right)$ are uniquely characterized by the fact $\left(P, \nabla_{P}\right)$ is "Frobenius invariant" (in some suitable sense) with respect to $\Phi_{N}^{\log }$.

Moreover, there is an open p-adic formal substack $\mathscr{C}^{\text {ord }} \subseteq \mathscr{C}$ of "ordinary points" of the curve. The open formal substack $\mathscr{C}^{\text {ord }} \subseteq \mathscr{C}$ is dense in every fiber of $\mathscr{C}$ over $\overline{\mathcal{N}}_{g, r}^{\text {ord }}$. Also, there is a unique canonical Frobenius lifting

$$
\Phi_{\mathscr{C}}^{\log }:\left(\mathscr{C}^{\log }\right)^{\text {ord }} \rightarrow\left(\mathscr{C}^{\log }\right)^{\text {ord }}
$$

which is $\bar{\Phi}_{N}^{\text {log }}$-linear and compatible with the Hodge section of the canonical indigenous bundle $\left(P, \nabla_{P}\right)$. Finally, $\Phi_{\mathscr{C}}^{10 g}$ and $\Phi_{\mathcal{N}}^{\log }$ have various functoriality properties. such as functoriality with respect to "log admissible coverings of $\mathscr{C}^{\log }$ " and with respect to restriction to the boundary of $\bar{M}_{g, r}$.

This Theorem is an amalgamation of Theorem 2.8 of Chapter III and Theorem 2.6 of Chapter V. In some sense all other results in this paper are formal consequences of the above Theorem. For instance,

Corollary 0.2. The Frobenius lifting $\Phi_{N}^{10 g}$ allows one to define canonical affine local coordinates on $\mathcal{M}_{g, r}$ at an ordinary point $\alpha$ valued in $k$, a perfect field of characteristic $p$. These coordinates are well-defined as soon as one chooses a quasiconformal equivalence class to which $\alpha$ belongs. Also. at a point $\alpha \in \bar{M}_{g, r}(k)$ corres ponding to a totally degenerate curve, $\Phi_{N}^{\log }$ defines canonical multiplicative local coor. dinates.

This Corollary follows from Chapter III, Theorem 3.8 and Definition 3.13. 
Let $\alpha \in \overline{\mathcal{N}}_{g, r}^{\text {ord }}(A)$, where $A=W(k)$, the ring of Witt vectors with coefficients in a perfect field of characteristic $p$. If $\alpha$ corresponds to a morphism Spec $(A) \rightarrow \overline{\mathcal{N}}_{g, r}^{\text {ord }}$ which is Frobenius equivariant (with respect to the natural Frobenius on $A$ and the Frobenius lifting $\Phi_{\mathcal{N}}^{\log }$ on $\overline{\mathcal{N}}_{g, r}^{\text {ord }}$, , then we call the curve corresponding to $\alpha$ canonical. Let $K$ be the quotient field of $A$. Let $\mathrm{GL}_{2}^{ \pm}(-)$be the group scheme which is the quotient of $\mathrm{GL}_{2}(-)$ by $\{ \pm 1\}$.

Theorem 0.3. Once one fixes a $k$-valued $\alpha_{0}$ of $\overline{\mathcal{N}}_{g, r}^{\text {ord }}$, there is a unique canonical $\alpha \in \overline{\mathcal{N}}_{g, r}^{\text {ord }}(A)$ that lifts $\alpha_{0}$. Moreover, if a curve $X^{\mathrm{log}} \rightarrow \operatorname{Spec}(A)$ is canonical, it admits

(1) A canonical dual crystalline (in the sense of [Falt], §2) Galois representation $\rho: \pi_{1}\left(X_{K}\right) \rightarrow \mathrm{GL}_{2}^{ \pm}\left(\mathbb{Z}_{p}\right)$ (which satisfies certain properties);

(2) A canonical log p-divisible group $G^{\log }$ (up to $\{ \pm 1\}$ on $X^{\log }$ whose Tate module defines the representation $\rho$ :

(3) A canonical Frobenius lifting $\Phi_{X}^{\log }:\left(X^{\text {log }}\right)^{\text {ord }} \rightarrow\left(X^{\log }\right)^{\text {ord }}$ over the ordinary locus (which satisfies certain properties).

Moreover, if a lifting $X^{\mathrm{log}} \rightarrow \operatorname{Spec}(A)$ of $\alpha_{0}$ has any one of these objects (1) through (3) (satisfying various properties), then it is canonical.

This Theorem results from Chapter III, Theorem 3.2, Corollary 3.4; Chapter IV, Theorem 1.1, Theorem 1.6, Definition 2.2, Proposition 2.3, Theorem 4.17.

The case of curves with ordinary reduction modulo $p$ which are not canonical is more complicated. Let us consider the universal case. Thus, let $S^{\log }=$ $\left(\overline{\mathcal{N}}_{g, r}^{\text {ord }}\right)^{\log }$; let $f^{\log :} X^{\log } \rightarrow S^{\log }$ be the universal $r$-pointed stable curve of genus $g$. Let $T^{\log } \rightarrow S^{\log }$ be the finite covering (log étale in characteristic zero) which is the Frobenius lifting $\Phi_{N}^{\log }$ of Theorem 0.1. Let $P^{\log } \rightarrow S^{\log }$ be the inverse limit of the coverings of $S^{\log }$ which are iterates of the Frobenius lifting $\Phi_{\mathcal{N}}^{\log }$. Let $X_{T}^{\log }=X^{\log } \times{ }_{S^{\log }} T^{\log } ; X_{P}^{\log }=X^{\log } \times{ }_{S^{\log }} P^{\log }$. We would like to consider the arithmetic fundamental groups

$$
\prod_{1} \stackrel{\text { def }}{=} \pi_{1}\left(\left(X_{T}^{\mathrm{log}}\right) \mathbf{Q}_{p}\right) ; \quad \prod_{\infty}^{\stackrel{\text { def }}{=}} \pi_{1}\left(\left(X_{P}^{\mathrm{log}}\right) \mathbf{Q}_{p}\right)
$$

Unlike the case of canonical curves, we do not get a canonical Galois representation of $\Pi_{1}$ into $\mathrm{GL}_{2}^{ \pm}\left(\mathbb{Z}_{p}\right)$. Instead, we have the following

Theorem 0.4. There is a canonical Galois representation 


$$
\rho_{\infty}: \mathbb{\Pi}_{\infty} \rightarrow \mathrm{GL}_{2}^{ \pm}\left(\mathbb{Z}_{p}\right)
$$

Moreover, the obstruction to extending $\rho_{\infty}$ to $\mathbb{I}_{1}$ is nontrivial and is measured precisely by the extent to which the canonical affine coordinates (of Corollary 0.2) are nonzero. Also, there is a ring $\mathscr{D}_{S}^{\text {Gal }}$ with a continuous action of $\pi_{1}\left(T_{Q_{p}}^{\log }\right)$ such that we have a canonical dual crystalline representation

$$
\rho_{1}: \mathbb{I}_{1} \rightarrow \mathrm{GL}_{2}^{ \pm}\left(\mathscr{D}_{S}^{\text {Gal }}\right)
$$

(i.e., this is a twisted homomorphism, with respect to the action of $\Pi_{1}$ (acting through $\pi_{1}\left(T_{\mathbf{Q}_{p}}^{\mathrm{log}}\right)$ ) on $\left.\mathscr{D}_{S}^{\mathrm{Gal}}\right)$. Finally, the ring $\mathscr{D}_{S}^{\mathrm{Gal}}$ has an augmentation $\mathscr{D}_{S}^{\mathrm{Gal}} \rightarrow \mathbb{Z}_{p}$ which is $\mathbb{I}_{\infty}$-equivariant (for the trivial action on $\mathbb{Z}_{p}$ ) and which is such that after restricting to $\mathbb{I}_{\infty}$, and base changing by means of this augmentation, $\rho_{1}$ reduces to $\rho_{\infty}$.

This follows from Chapter V, Theorems 1.4 and 1.7 .

All along, we note that when one specializes the theory to the case of elliptic curves, one recovers the familiar classical theory of Serre-Tate. For instance, the definitions of "ordinary curves" and "canonical liftings" specialize to the objects with the same names in Serre-Tate theory. The $p$-adic canonical coordinates on the moduli stack $\mathcal{M}_{g, r}$ (Corollary 0.2 ) specialize to the Serre-Tate parameter. The Galois obstruction to extending $\rho_{\infty}$ to a representation of $\mathbb{M}_{1}$ specializes to the obstruction to splitting the well-known exact sequence of Galois modules that the $p$-adic Tate module of an ordinary elliptic curve fits into.

For more detailed accounts of the results in each Chapter, we refer to the introductory sections at the beginnings of each of the Chapters. In the rest of this introductory Chapter, we explain the relationship between the $p$-adic case and the classically known complex case.

Acknowledgements: I would like to thank Prof. Barry Mazur of Harvard University for providing the stimulating environment (during the Spring of 1994) in which this paper was written. Also, I would like to thank both Prof. Mazur and Prof. Yasutaka Ihara (of RIMS, Kyoto University) for their efforts in assisting me to publish this paper, and for permitting me to hold lecture series at Harvard (Spring of 1994) and RIMS (Fall of 1994), respectively, during which I discussed the contents of this paper. Finally, I would like to thank Prof. Ihara for informing me of the theory of [Ih], [Ih2], [Ih3], and [Ih4]. 
This theory anticipates many aspects of the theory of the present paper (especially, the discussion of Frobenius liftings and pseudo-correspondences in Chapters III and IV). On the other hand, the techniques and point of view of Prof. Ihara's theory differ substantially from those of the present paper. Moreover, from a rigorous, mathematical point of view, the main results of Prof. Ihara's theory neither imply nor are implied by the main results of the present paper. However, it is the author's subjective opinion that philosophically, the motivation behind Prof. Ihara's theory was much the same as that of the author's.

\section{$\S 1$. Review of the Complex Theory}

In order to explain the meaning of the main results of this paper, it is first necessary to review the complex theory of uniformization in a fashion that makes the generalization to finite primes more natural. This is the goal of the present Section. Since all of the material is "standard" and "well-known", we shall, of course, omit proofs, instead citing references for major results. We shall say that a Riemann surface $X$ is of finite type if it can be obtained by removing a finite number of points $p_{1}, \ldots, p_{r}$ from a compact Riemann surface $Y$ of genus $g$. Note that in this case, $Y$ and $\left\{p_{1}, \ldots, p_{r}\right\}$ are uniquely determined up to isomorphism. We shall say that the Riemann surface of finite type $X$ is hyperbolic (respectively, parabolic; elliptic) if $2 g-2+r \geq 1$ (respectively, $2 g-2+r$ $=0 ; 2 g-2+r<0)$. In this paper, we shall be concerned exclusively with Riemann surfaces of finite type (and their uniformizations). This is because it is precisely these Riemann surfaces which correspond to algebraic objects. Also, we shall mainly be concerned with the hyperbolic case, since this is the most difficult. Indeed, from the point of view of the theory of uniformization and moduli, the elliptic case is completely trivial, and the parabolic case (although nontrivial) is relatively easy and explicit.

In some sense, the theme of our review of the classical complex theory is that in most cases, there are two ways to approach results: the "classical" and the "quasiconformal". Typically, the classical approach was known earlier, and is more geometric and intuitive. On the other hand, the classical approach has the drawback of producing theories and results that are only real analytic, rather than holomorphic in nature. By contrast the quasiconformal approach, which was pioneered by Ahlfors and Bers, tends to give rise to holomorphic structures and results naturally. It is thus natural that the connection between the "quasiconformal approach" and the $p$-adic theory should be much more natural and transparent. 


\section{Beltrami Differemtials}

Let $X$ be a Riemann surface (not necessarily of finite type). Let us consider the complex line bundle $\tau_{X} \otimes \bar{\omega}_{X}$ on $X$, where $\bar{\omega}_{X}$ is the complex conjugate bundle to the canonical bundle $\omega_{X}$, and $\tau_{X}$ is the tangent bundle. Note that if $s$ is a section of $\tau_{X} \otimes \bar{\omega}_{X}$ over $X$, then we can consider its $L^{\infty}$-norm $\left\|_{s}\right\|_{\infty}$, since the transition functions of $\tau_{X} \otimes \bar{\omega}_{X}$ have complex absolute value 1. A Beltrami differential $\mu$ on $X$ is a measurable section of the line bundle

$$
\tau_{X} \otimes \bar{\omega}_{X}
$$

such that $\|\mu\|_{\infty}<1$.

Why the bundle $\tau_{X} \otimes \bar{\omega}_{X}$ ? The reason is that this bundle is closely connected with the moduli of the Riemann surface $X$. Indeed, let us consider an arbitrary $\mathscr{C}^{\infty}$ section $\mu$ of $\tau_{X} \otimes \bar{\omega}_{X}$. Now since $\tau_{X}$ has the structure of a holomorphic line bundle, we have a $\bar{\partial}$ operator on $\tau_{X}$. If we look at global $\mathscr{C}^{\infty} \mathrm{sec}$ tions, this gives us a complex

$$
\mathscr{C}^{\infty}\left(X, \tau_{X}\right) \stackrel{\bar{\partial}}{\rightarrow} \mathscr{C}^{\infty}\left(X, \tau_{X} \otimes \bar{\omega}_{X}\right)
$$

which computes the analytic cohomology of $\tau_{X}$. If $X$ is, for instance, compact, then this analytic cohomology coincides with the cohomology in the Zariski topology of the algebraic tangent bundle. Thus, for $X$ compact and hyperbolic, the above complex has cohomology groups $H^{0}=0$, and $H^{1}=H^{1}\left(X, \tau_{X}\right)$, which is well-known to be the space of infinitesimal deformations of $X$. Moreover, if $X$ is compact of genus $g \geq 2$, and $\mathcal{M}_{g}$ is the moduli stack of curves of genus $g$, then $H^{1}\left(X, \tau_{X}\right)$ is precisely the tangent space to $\mathcal{M}_{g}$ at the point defined by $X$.

At any rate, (for $X$ arbitrary) we have a natural surjection

$$
\mathscr{C}^{\infty}\left(X, \tau_{X} \otimes \bar{\omega}_{X}\right) \rightarrow H^{1}\left(X, \tau_{X}\right)
$$

Thus, the image of $\mu$ under this surjection defines an infinitesimal deformation of the complex structure of $X$. This establishes the relationship between sections of

$$
\tau_{X} \otimes \bar{\omega}_{X}
$$

and the moduli of $X$. The reason for considering measurable, rather than just 
$\mathscr{C}^{\infty}$, sections is that it is easier to obtain solutions to a certain differential equation, the Beltrami equation, when one works in this greater generality.

\section{The Beltrami Equation}

Having established the relationship between sections of $\tau_{X} \otimes \bar{\omega}_{X}$ and infinitesimal deformations, we now would like to integrate - i.e., to "give a reciprocity law" - that assigns to a section $\mu$ of $\tau_{X} \otimes \bar{\omega}_{X}$ not just an infinitesimal deformation of $X$, but an actual new Riemann surface, i.e., a new complex structure on the topological manifold underlying $X$. To do this, we consider the Beltrami equation

$$
\bar{\partial} f=\mu \cdot \partial f
$$

which we regard as a differential equation in the unknown function $f$. It is a nontrivial result (proven, for instance, in [Lehto2] ) that when $\mu$ is a Beltrami differential, there exist local $L^{2}$ solutions $f$ to the Beltrami equation that are homeomorphisms (where they are defined). Such functions $f$ are called quasiconformal (with dilatation $\mu$ ). If $f$ and $g$ (defined on some open set $U \subseteq X$ ) are both quasiconformal with the same dilatation $\mu$, then it is easy to see that $\bar{\partial}$ applied to $f: g^{-1}$ (in the distributional sense) is zero. That is, $f=h, g$ for some biholomorphic function $h$. Thus, up to composition with a biholomorphic function. quasiconformal solutions to the Beltrami equation are unique.

With these observations, we can define a new complex structure on $X$ associated to a Beltrami differential $\mu$ as follows. Let us call the resulting Riemann surface $X_{\mu}$. Thus, the underlying topological manifold of $X_{\mu}$ is the same as that of $X$. On an open set $U \subseteq X$, we take a local quasiconformal function $f$ of dilatation $\mu$, and define it to be a holomorphic function on $X_{\mu}$. By the essential uniqueness of solutions to the Beltrami equation, everything is well-defined, and so we obtain a new global Riemann surface $X_{\mu}$. Thus, the assignment

$$
\mu \mapsto X_{\mu}
$$

is the fundamental "reciprocity law" that we are looking for.

\section{The Series Expansion of a Quasiconformal Function}

In order to really understand the Beltrami equation, it is useful to look at the explicit representation of its solutions as series "in $\mu$ " (as in [Lehto], pp. 25-27). We begin by considering Cauchy's integral formula: 


$$
f(z)=\frac{1}{2 \pi i} \int_{\partial D} \frac{f(\zeta) d \zeta}{\zeta-z}-\frac{1}{\pi} \iint_{D} \frac{\bar{\partial} f(\zeta) d \xi d \eta}{\zeta-z}
$$

for a function $f$ with $L^{1}$ derivatives on an open disk $D$ in the complex plane. Thus, if $f$ (and its $L^{1}$ derivatives) are defined on all of $\mathbb{C}$, and $f(z) \rightarrow 0$ as $z \rightarrow$ $\infty$, then we obtain

$$
f(z)=T \bar{\partial} f
$$

where $T$ is the operator on $\mathscr{C}^{\infty}$ functions $\omega$ with compact support given by

$$
(T \omega)(z)=-\frac{1}{\pi} \iint_{C} \frac{\omega(\zeta) d \xi d \eta}{\zeta-z}
$$

Put another way, (from the point of view of the theory of pseudodifferential operators) $T$ is the parametrix for the elliptic differential operator $\bar{\partial}$. If we define the Hilbert transformation II by

$$
(H \omega)(z)=-\frac{1}{\pi} \iint_{\mathbb{C}} \frac{\omega(\zeta) d \xi d \eta}{(\zeta-z)^{2}}
$$

then we obtain that $\partial T=H$. Also, it can be shown that $\partial$ and $\bar{\partial}$ commute with both $T$ and $H$.

Now let us suppose that $\mu$ is a Beltrami differential on $\mathbb{C}$ (say, with compact support), and that $f$ is quasiconformal on $\mathbb{C}$ with dilatation $\mu$. Then $f$ is holomorphic at infinity, and so, after normalization, in a neighborhood of infinity, it looks like

$$
f(z)=z+\sum_{n \geq 1} b_{n} z^{-n}
$$

for some $b_{n} \in \mathbb{C}$. Thus, $f(z)-z$ goes to 0 as $z \rightarrow \infty$, so we obtain that

$$
\begin{aligned}
\partial f(z) & =1+\partial\{f(z)-z\} \\
& =1+\partial T \bar{\partial}\{f(z)-z\} \\
& =1+H \bar{\partial} f(z)
\end{aligned}
$$

Thus, since $\bar{\partial} f=\mu \cdot \partial f$, it follows that

$$
\bar{\partial} f=\mu+\mu \cdot H \bar{\partial} f
$$


This integral equation has the formal solution

$$
\bar{\partial} f=\sum_{i \geq 0}(\mu \cdot H)^{i} \mu
$$

which converges in $L^{2}$ because

(1) it can be shown that $H$ extends to an isometry $L^{2} \rightarrow L^{2}$;

(2) since $\mu$ is a Beltrami differential, $\|\mu\|_{\infty}<1$ (which thus explains this part of the definition of a Beltrami differential).

Thus, applying the operator $T$, we get the series solution

$$
f(z)=z+T\left\{\sum_{i \geq 0}(\mu \cdot H)^{\imath} \mu\right\}
$$

to the Beltrami equation.

From our point of view, this series solution has two important consequences. First of all, the set of all possible $\mu$ clearly forms an open subset of a (rather large) complex vector space (i.e., the space of measurable sections of $\tau_{X}$ $\left.\otimes \bar{\omega}_{X}\right)$. Thus, relative to the complex structure of this complex vector space, the series solution makes it clear that $f$ depends holomorphically on $\mu$. Second, it computes the infinitesimal change in $f$ as $\mu$ varies to first order. Namely, this term is given by $\phi \stackrel{\text { def }}{=} T(\mu)$. Note that

$$
\bar{\partial} \phi=\mu
$$

It turns out that this result - that $\bar{\partial}$ applied to the infinitesimal change $\phi$ in the solution to the Beltrami equation gives us back $\mu$-holds for arbitrary Beltrami differentials $\mu$. (See, e.g., [Gard], p. 72).

The reason why this observation is interesting is as follows. Suppose, for simplicity, that $\mu$ is $\mathscr{C}^{\infty}$. Let $\mathcal{U}$ be an open covering of $X$ such that the intersection of any finite collection of open sets in $\mathcal{U}$ is Stein. Then by considering the standard isomorphism between the $\breve{C}$ ech cohomology (with respect to $U$ ) and the $\bar{\partial}$-cohomology of $\tau_{X}$, it thus follows that the infinitesimal deformation $X_{\epsilon} \cdot \mu$ (where $\epsilon$ is "small") in the complex structure of $X$ given by solving the Beltrami equation is precisely the same as the infinitesimal deformation given by mapping $\mu$ to $H^{1}\left(X, \tau_{X}\right)$ via the surjection

$$
\mathscr{C}^{\infty}\left(X, \tau_{X} \otimes \bar{\omega}_{X}\right) \rightarrow H^{1}\left(X, \tau_{X}\right)
$$


considered previously. This completes the justification of the claim that the assignment $\mu \mapsto X_{\mu}$ is an "integrated version" of the "infinitesimal reciprocity law"

$$
\mathscr{C}^{\infty}\left(X, \tau_{X} \otimes \bar{\omega}_{X}\right) \rightarrow H^{1}\left(X, \tau_{X}\right)
$$

that follows just from the definition of the $\bar{\partial}$-cohomology of $\tau_{X}$.

\section{Uniformization of Hyperbohic Riemanm Surfices}

Let $X$ be a hyperbolic Riemann surface. Let $\widetilde{X}$ be its universal covering space. Thus, $\widetilde{X}$ inherits a natural complex structure from $X$. Then one of the most basic results in the field is that we have an isomorphism of Riemann surfaces

$$
\widetilde{X} \cong H
$$

where $H$ is the upper half plane. By considering the covering transformations of $H \rightarrow X$, we get a homomorphism (well-defined up to conjugation)

$$
\rho: \pi_{1}(X) \rightarrow \operatorname{Aut}(H) \subseteq \mathrm{PSL}_{2}(\mathbb{R})
$$

which we call the canonical representation of $X$.

There are (at least) two ways to prove this result. The first approach is the classical approach, and goes back to Koebe's work in the early twentieth century. It involves considering Green's functions $G(-,-)$ on $\widetilde{X}$. There is an intrinsic, a priori definition of Green's functions, which is not important for us here. A posteriori, that is, once one knows that $\widetilde{X} \cong H$, we can pull-back the hyperbolic metric

$$
\frac{d x^{2}+d y^{2}}{y^{2}}
$$

on $H$ to $\widetilde{X}$, so that we obtain a hyperbolic distance function of $\widetilde{X}$. Then $G(x, y)$ (for $x, y \in \widetilde{X}$ ) is given by the logarithm of the hyperbolic distance between $x$ and $y$. One can find a detailed exposition of this approach in [FK].

The second approach (which is more relevant to the $p$-adic case) is the 
approach of Bers ([Bers]). Suppose that $X$ is obtained by removing $r$ points from a compact Riemann surface $Y$ of genus $g$. Then one first observes that there exists a Riemann surface $X^{\prime}$ which is obtained by removing $r$ points from a compact Riemann surface of genus $g$ and whose universal covering space $\widetilde{X}^{\prime}$ is isomorphic to $H$. Then one constructs (from purely elementary considerations) a quasiconformal homeomorphism $X^{\prime} \cong X$. This quasiconformal homeomorphism defines a Beltrami differential $\mu$ on $X^{\prime}$, which we can pull back to $\widetilde{X}^{\prime} \cong H$ to obtain a Beltrami differential $\mu_{H}$ on $H$. By reflection, one extends $\mu_{H}$ to a Beltrami differential $\tilde{\mu}$ on $\mathbb{C}$. Then we solve the Beltrami equation for $\tilde{\mu}$ on $\mathbb{C}$ so that we obtain a quasiconformal homeomorphism

$$
f_{\tilde{\mu}}: \mathbf{C} \rightarrow \mathbf{C}
$$

which goes to infinity at infinity. Let $\Gamma^{\prime}$ be the group of Möbius transformation of $H$ defined by the covering transformations of $\widetilde{X}^{\prime}$ over $X^{\prime}$. Thus, $H / \Gamma^{\prime} \cong$ $X^{\prime}$. Then it follows from the uniqueness of solutions to the Beltrami equation that

$$
\Gamma \stackrel{\text { def }}{=} f_{\tilde{u}} r \Gamma^{\prime}=f_{\tilde{\mu}}^{-1}
$$

forms a group of Möbius transformation of $\mathbb{C}$. Moreover, from the reflection symmetry of $\tilde{\mu}$, it follows that $f_{\tilde{\mu}}$ preserves the real axis, and hence so does $\Gamma$. It thus follows that $H / \Gamma$ is a Riemann surface of finite type, and, by the definition of $\mu$, that $H / \Gamma \cong X$. This completes the proof.

It turns out that it is this approach of uniformizing a single Riemann surface (for each $g, r$ ) and then "parallel transporting" the result over the rest of the moduli space that will carry over to the $p$-adic case.

\section{Uniformization of Moduli Stacks of Hyperbolic Riemann Surfaces}

Let $\mathcal{M}_{g, r}$ be the moduli stack of $r$-pointed smooth algebraic curves of genus $g$ over $\mathbb{C}$. Let $\widetilde{M}_{g, r}$ be its universal covering space. Then the problem of uniformization of moduli is to give an explicit representation of $\widetilde{M}_{g, r}$. From the point of view of the Beltrami equation, this amounts to finding a small, finite-dimensional subspace $T$ of the space of Beltrami differentials $\mu$ such that the assignment $\mu \mapsto X_{\mu}$ defines a covering space map $T \rightarrow \mathcal{M}_{g, r}$.

We begin by fixing a "base point" of $\mathcal{M}_{g, r}$, which corresponds to a hyperbolic Riemann surface $X$. Let $\mathcal{M}(X)$ be the space of Beltrami differentials on $X$. 
Let $Q$ be the space of holomorphic quadratic differentials on $X$ with at most simple poles at the punctured points. Then there are two approaches to defining morphisms from open subsets of $Q$ into spaces of Beltrami differentials. The first approach is that of Teichmüller. In this approach, if $\phi \in Q$, we define a norm

$$
\|\phi\| \stackrel{\text { def }}{=} \int_{X}|\phi|
$$

Let $V \subseteq Q$ be the set of $\phi$ with $\|\phi\| \leq 1$. Then Teichmüller's uniformization map, for (nonzero) $\phi \in V$, is given by

$$
\phi \mapsto \mu_{\phi} \stackrel{\text { def }}{=}(\|\phi\|) \frac{\bar{\phi}}{|\phi|}
$$

where $\bar{\phi} \in \Gamma\left(X, \bar{\omega}_{X}^{\otimes 2}\right)$ is the complex conjugate of $\phi$. It is easy to see that $\mu_{\phi}$ defines a Beltrami differential on $X$. Thus, we get a morphism $V \rightarrow \mathcal{M}(X)$. If we compose $\phi \mapsto \mu_{\phi}$ with $\mu \mapsto X_{\mu}$, we get a morphism

$$
V \rightarrow M_{g, r}
$$

The main result of Teichmüller theory (see, e.g., [Gard], Chapter 6) is that this morphism induces an isomorphism of $V$ onto $\widetilde{M}_{g, r}$. One advantage of this approach is that it admits a very satisfying geometric interpretation in terms of a foliation on $X$ induced by $\phi$ and deforming $X$ into $X_{\mu_{\phi}}$ by deforming a canonical coordinate arising from the foliation. The main disadvantage of this approach from our point of view, however, is that the morphism $\phi \mapsto \mu_{\phi}$ is neither holomorphic nor anti-holomorphic. Thus, it seems hopeless to try to find an algebraic version of Teichmüller's map.

On the other hand, Bers' approach is as follows. Since we now know that $X$ can be uniformized by the upper half plane, let $v_{X}$ be the hyperbolic volume element on $X$ induced by the hyperbolic volume element

$$
v_{H}=\frac{d x \wedge d y}{y^{2}}
$$

on the upper half plane. Let $X^{\mathrm{c}}$ be the conjugate Riemann surface to $X$. That is, the underlying topological manifold of $X^{\mathrm{c}}$ is the same as that of $X$, but the holomorphic functions on $X^{c}$ are exactly the anti-holomorphic functions on $X$. Suppose that $\phi \in Q$. Then by conjugating the "input variable," we obtain that $\phi$ defines a section $\phi^{\mathrm{c}}$ of $\bar{\omega}_{\lambda^{c}}^{\otimes 2}$. Now define 


$$
\mu_{\phi} \stackrel{\text { def }}{=} \frac{-2 \phi^{\mathrm{c}}}{v_{X^{\mathrm{c}}}}
$$

Then for some appropriate (see [Gard], pp. 100-104) open set $V \subseteq Q$, this $\mu_{\phi}$ defines a Beltrami differential on $X^{\mathrm{c}}$. Integrating, we get a Riemann surface $X_{\mu}^{\mathrm{c}}$. Then the assignment $\phi \mapsto X_{\mu}^{\mathrm{c}}$ defines a morphism

$$
V \rightarrow M_{g, r}^{c}
$$

where the superscript "c" denotes the conjugate complex manifold. This morphism induces an isomorphism of $V$ onto $\widetilde{M}_{g, r}^{c}$ ([Gard], p. 101). The important thing here is that the correspondence $\phi \mapsto \mu_{\phi}$ is holomorphic. Since $\mu \mapsto X_{\mu}^{\mathrm{c}}$ is always holomorphic, it thus follows that the isomorphism $V \cong \widetilde{M}_{g, r}^{c}$ is biholomorphic. Put another way, we have a holomorphic embedding

$$
\mathscr{B}: \widetilde{M}_{g, r} \hookrightarrow Q^{\mathrm{c}}
$$

which is called the Bers embedding. This embedding will be central to our en tire treatment of the complex theory, and its $p$-adic analogue will be central to our treatment of the $p$-adic theory.

\section{Quasidisks and the Bers Embedding}

One can also define the Bers embedding in terms of Bers' simultaneous uniformization and Schwarzian derivatives. For details, see [Gard], pp. 100-101. To do this, we fix an isomorphism of $\widetilde{X}$ with $H$. Let $H^{c}$ be the lower half plane. Thus, if $H$ uniformizes $X$, then $H^{\mathrm{c}}$ naturally uniformizes $X^{\mathrm{c}}$. Let $\Gamma$ be the group of Möbius transformiations of $\mathbb{C}$ which are the covering transformations for $H=$ $\widetilde{X} \rightarrow X$. Then we may think of the space $M\left(X^{c}\right)$ of Beltrami differentials on $X^{\mathrm{c}}$ as the space of Beltrami differentials on $H^{\mathrm{c}}$ which are invariant under $\Gamma$. Let $\mu \in M\left(X^{\mathrm{c}}\right)$. Let $f^{\mu}: \mathbb{C} \rightarrow \mathbb{C}$ be the unique quasiconformal homeomorphism which fixes 0 and 1 , goes to infinity at infinity, has Beltrami coefficient $\mu$ on $H^{\mathrm{c}}$ and is conformal on $H$. Let $\Gamma^{\mu}=f^{\mu}=\Gamma=\left(f^{\mu}\right)^{-1}$. Then it follows from the uniqueness of solutions to the Beltrami equation that $\Gamma^{\mu}$ forms a group of Möbius transformations of $\mathbf{C}$. Moreover, we have conformal isomorphisms

$$
f^{\mu}\left(H^{\mathrm{c}}\right) / \Gamma^{\mu} \cong X_{\mu}^{\mathrm{c}} ; \quad f^{\mu}(H) / \Gamma^{\mu} \cong X
$$

It follows that if we take the Schwarzian derivative of the conformal "quasidisk" 
embedding

$$
\left.f^{\mu}\right|_{H}: H \hookrightarrow \mathbb{C}
$$

we get a $\Gamma$-invariant quadratic differential on $H$, hence a quadratic differential $\phi$ (with at most simple poles at the punctures) on $X$. The content of the Lemma of Ahlfors-Weill ([Gard], p. 100) is that the assignment:

$$
X_{\mu}^{\mathrm{c}} \mapsto \phi
$$

is equal to $\mathscr{B}^{\mathrm{c}}$ : $\widetilde{M}_{g, r}^{\mathrm{c}} \hookrightarrow Q$. On the one hand, this description of the Bers embedding is geometrically more satisfying than the definition given in the previous subsection, but it has the disadvantage that it obscures the relationship between the hyperbolic and parabolic cases. So far we have been mainly discussing the hyperbolic case, but we shall discuss the parabolic case later.

\section{The Infinitesimall Form of the Madunlr Uniformizations}

Often it is useful to express modular uniformizations in their infinitesimal form, as metrics. On the one hand, the global uniformizations can always be essentially recovered by integrating the metrics, and on the other hand, metrics, being local in nature, can often be studied more easily.

In the Teichmüller case, if $K$ is defined by

$$
\|\phi\|=\frac{K-1}{K+1}
$$

then one obtains a distance function on $\widetilde{M}_{g, r}$, given by

$$
d\left(X, X_{\mu_{\phi}}\right)=\frac{1}{2} \log (K)
$$

which turns out to be equal to the general hyperbolic distance introduced by Kobayashi for an arbitrary hyperbolic complex manifold (see [Gard], Chapter 7 , for an exposition). The infinitesimal form of this distance is given by the norm $\|\phi\|=\int_{X}|\phi|$ on quadratic differentials (see [Royd]).

We shall be more interested in the case of the Bers embedding

$$
\mathscr{B}: \widetilde{M}_{g . r} \hookrightarrow Q^{\mathrm{c}}
$$


By using the hyperbolic volume form $v_{X}$ on $X$, we obtain the Weil-Petersson inner product:

$$
\langle\phi, \phi\rangle \stackrel{\text { def }}{=} \int_{X} \frac{\phi \cdot \bar{\phi}}{v_{X}}
$$

for $\phi, \phi \in Q$. It is a result of Weil and Ahlfors that the resulting metric, called the Weil-Petersson metric on $\mathcal{M}_{g, r}$, is Kähler. Moreover, if we differentiate $\mathscr{B}$, we get at $X$, a map on tangent spaces

$$
d \mathscr{B}: Q^{\vee} \rightarrow Q^{\mathrm{c}}
$$

whose inverse is exactly the morphism $Q^{\mathbf{c}} \rightarrow Q^{\vee}$ defined by the Weil-Petersson inner product. Finally, the coordinates obtained from the Bers embedding are canonical coordinates for the Weil-Petersson metric ([Royd]). (We shall review the general theory of canonical coordinates associated to a real analytic Kähler metric in $\S 2$.)

It turns out that it is precisely the p-adic analogue of the Weil-Petersson metric that will play a central role in this paper.

\section{Coordinates of Degeneration}

While the Bers coordinates are useful for understanding what happens in the interior of $\mathcal{M}_{g, r}$, they are not so useful for understanding what happens as one goes out to the boundary, that is, as the Riemann surface degenerates to a Riemann surface with nodes. To study this sort of degeneration, one fixes a decomposition of the Riemann surface into "pants", which are topologically equivalent to an open disk with two smaller disks in the interior removed. For a detailed description of the theory of pants and the coordinates they define, we refer to [Abikoff], Chapter 2. In summary, what happens is the following. Let $X$ be a hyperbolic Riemann surface (of genus $g$ with $r$ punctures), with a decomposition into pants. We shall call the curves on $X$ which occur in the boundary of the pants partition curves. There are exactly $3 g-3+r$ partition curves, $\alpha_{1}, \ldots, \alpha_{3 g-3+r}$. We assume that this decomposition is "maximal" in the sense that each partition curve is a simple closed geodesic (in the hyperbolic metric on $X$ ). Then it turns out that the isomorphism class of $X$ as a Riemann surface is completely determined by $3 g-3+r$ complex numbers $\zeta_{l}=l_{\imath} e^{i \theta} \quad(i=1$. $\ldots, 3 g-3+r)$, one for each partition curve. Basically $l_{t}$ describes the circumference of the partition curve $\alpha_{\imath}$, while $\theta_{\imath}$ describes the angle of twisting involved in gluing together the boundary curves of two neighboring pants to form $\alpha_{\imath}$. These coordinates $\zeta_{\imath}$ are called the Fenchel-Nielsen coordinates of $X$. The 
degeneration corresponding to pinching $\alpha_{i}$ to a node is given by $l_{i} \rightarrow 0$. This degeneration respects the hyperbolic metrics involved: that is, if a family of smooth $X_{t}$ degenerates to a nodal Riemann surface $Z$, then the hyperbolic metrics on the $X_{t}$ degenerate to the hyperbolic metric on $Z$ (given by taking the hyperbolic metric on the smooth subsurface of $Z$ which is the complement of the nodes). Thus, the Fenchel-Nielsen coordinates have the virtue of admitting a very satisfying differential-geometric description (as just summarized), but the disadvantage of not being holomorphic.

On the other hand, one can define holomorphic coordinates (as in [Wolp]), as follows. Recall the quasidisk description of the Bers embedding. Thus, we had a $\mu \in M\left(X^{c}\right)$, and a quasiconformal homeomorphism $f^{\mu}: \mathbb{C} \rightarrow \mathbb{C}$, together with a new group of Möbius transformations $\Gamma^{\mu}$. Then each $\alpha_{\imath}$ defines (by integration) an element $A_{i} \in \Gamma^{\mu}$. Up to conjugation, $A_{\imath}$ is of the form $z \mapsto m_{i}{ }^{\circ} z$ for some $m_{\imath} \in \mathbb{C}$ with $\left|m_{\imath}\right|>1$. This complex number $m_{\imath}$ is uniquely defined. Then the coordinates

$$
X_{\mu}^{\mathrm{c}} \mapsto\left(m_{1}, \ldots, m_{3 g-3+r}\right)
$$

are holomorphic in $\mu$. In [Wolp], the relationship between these coordinates and the Bers coordinates is studied. In these coordinates, the degeneration of $X_{\mu}^{c}$ corresponding to the case where the partition curve $\alpha_{\imath}$ is pinched to a node is given by $m_{l} \rightarrow 1$. It turns out that these coordinates are probably the best complex analogue to the "multiplicative parameters at infinity" that we construct in the $p$-adic case.

\section{The Parabolic Case}

So far we have mainly been discussing the case of hyperbolic Riemann surfaces, since this case is by far the most interesting. However, often it is very difficult to make explicit computations for hyperbolic Riemann surfaces. Thus, in order to get one's bearings, it is sometimes useful to consider the analogous constructions in the parabolic case, where explicit computations are much easier to carry out. Let $X$ be a parabolic Riemann surface. Then $X$ is either compact of genus 1, or it is isomorphic to the projective line minus two points. We shall mainly be interested in the compact case, where there are nontrivial moduli.

Thus, let $X$ be compact of genus 1 . Then one can carry out Teichmüller theory in this case (as in [Lehto], Chapter V, §6). One can also define a parabolic analogue of the Bers embedding, as follows. Namely, we simply copy the formula 


$$
\mu_{\phi} \stackrel{\text { def }}{=} \frac{-2 \phi^{\mathrm{c}}}{v_{\lambda^{r}}}
$$

of the hyperbolic case, except that we take $v_{X^{c}}$ to be the parabolic volume element (as opposed to the hyperbolic volume element) on $X^{\mathrm{c}}$, with $\int_{X^{\mathrm{c}}} v_{X^{\mathrm{c}}}=1$. Then one sees (as in [Lehto], p. 220) that one obtains a holomorphic embedding

$$
B_{1,0}: \widetilde{M}_{1,0} \hookrightarrow Q^{\mathrm{c}}
$$

whose image is an open disk $D \subseteq Q^{\mathrm{c}}$ of some radius. One can also define a Weil-Petersson metric on $\mathcal{M}_{1,0}$ by simply replacing the hyperbolic volume element used before by the parabolic volume element. A simple calculation then reveals that one obtains the standard hyperbolic metric on the open disk $D$. In particular, (just as in the hyperbolic case), the standard coordinate on $D$ is normal at 0 for the Weil-Petersson metric.

One thing that is interesting about this parabolic case is that even though the complex analytic stacks $\mathcal{M}_{1,0}$ and $\mathcal{M}_{1,1}$ are isomorphic, the "Bers theory" differs substantially in the two cases. For instance, the Bers embedding of $\widetilde{M}_{1,1}$ is far from being an open disk. In fact, (as the author was told by $\mathrm{C}$. McMullen) the boundary of this hyperbolic Bers embedding has lots of cusps. A computer-generated illustration of this boundary appears in [McM]. Also, it is not difficult to show that the Weil-Petersson metrics are quite different. This contrasts considerably with the "Teichmüller theory" of $\mathcal{M}_{1,0}$ and $\mathcal{M}_{1,1}$ : Indeed, since Teichmüller's metric always coincides with Kobayashi's intrinsic hyperbolic metric, it follows that the Teichmüller metrics of $\mathcal{M}_{1,0}$ and $\mathcal{M}_{1,1}$ coin cide.

\section{Real Curves}

A Riemann surface $X$ of finite type is called real if $X \cong X^{\mathrm{c}}$. In other words, this means that the $\dot{\mathbf{C}}$-valued point defined by $X$ in the algebraic stack $\left(\mathcal{M}_{g, r}\right)_{\mathbf{R}}$ (over $\operatorname{Spec}(\mathbf{R})$ ) is, in fact, defined over $\mathbf{R}$ (up to perhaps reordering the marked points). Various interesting properties of real Riemann surfaces (related to uniformization theory) are studied in [Falt2]. Many of these properties are obtained by looking at various one-dimensional real analytic submanifolds of a real $X$.

From our point of view, however, the notable fact about real hyperbolic Riemann surfaces $X$ is the following. Let $\phi: X \cong X^{c}$ be a holomorphic isomor- 
phism. For simplicity, suppose that there exists a point $x \in X$ such that $\phi(x)$ $=x^{\mathrm{c}}$, and that $\phi^{\mathrm{c}} \circ \phi=\mathrm{id}_{X}$. Fix an isomorphism $\widetilde{X} \cong H$. This induces an isomorphism $\widetilde{X}^{\mathrm{c}} \cong H^{\mathrm{c}}$. On the other hand, $\phi$ induces a holomorphic isomorphism $\tilde{\phi}$ : $H \rightarrow H^{\mathrm{c}}$. Let $C: H^{\mathrm{c}} \rightarrow H$ be the conjugation map. Let $\phi=C \backsim \tilde{\phi}$. Thus, $\phi$ is an anti-holomorphic automorphism of $H$. Now let $\mathbb{I}_{\mathbb{C}}=\pi_{1}(X, x)$. Since $X^{\mathrm{c}}$ has the same underlying topological space as $X$, we have $\mathbb{I}_{\mathbb{C}}=\pi_{1}\left(X^{\mathrm{c}}, x^{\mathrm{c}}\right)$. Thus, $\phi$ in duces an automorphism $\phi_{\Pi}$ of $\mathbb{\Pi}_{\mathbb{C}}$ of degree 2 . Let $\mathbb{\Pi}_{\mathbb{R}}$ be the extension

$$
1 \rightarrow \mathbb{\Pi}_{\mathbf{C}} \rightarrow \mathbb{M}_{\mathbb{R}} \rightarrow \mathrm{Gal}(\mathbb{C} / \mathbb{R}) \rightarrow 1
$$

which is the crossed product of $\mathbb{I}_{\mathrm{C}}$ with $\mathrm{Gal}(\mathbb{C} / \mathbb{R})$ given by letting the nontriv-

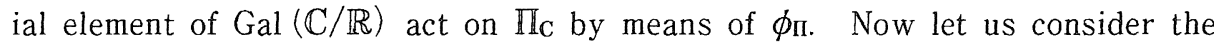
Lie group

$$
G(\mathbb{R}) \stackrel{\text { def }}{=}\left\{M \in \mathrm{GL}_{2}(\mathbb{R}) \mid \operatorname{det}(M)= \pm 1\right\} /\{ \pm 1\}
$$

Thus, $\mathrm{PSL}_{2}(\mathbb{R}) \subseteq G(\mathbb{R}) \subseteq \mathrm{GL}_{2}^{ \pm}(\mathbb{R})$, so we can write

$$
\rho_{\mathrm{C}}: \Pi_{\mathbb{C}} \rightarrow \mathrm{GL}_{2}^{ \pm}(\mathbb{R})
$$

for the canonical representation of $X$ (uniformized by the upper half plane $H$ ). Note that the full group $\mathrm{GL}_{2}^{ \pm}(\mathbb{R})$ acts on the upper half plane as follows: if $A=$ $\left(\begin{array}{ll}a & b \\ c & d\end{array}\right) \in \mathrm{GL}_{2}^{ \pm}(\mathbb{R})$, we let

$$
A(z)=\frac{a w+b}{c w+d}
$$

where $u^{\prime}=z$ (respectively, $w=\bar{z}$ ) if $\operatorname{det}(A)$ is positive (respectively, negative). Thus, the map defined by $A$ is a holomorphic (respectively, anti-holomorphic) automorphism of $H$ if $\operatorname{det}(A)$ is positive (respectively, negative). In particular, the anti-holomorphic automorphism $\phi: H \rightarrow H$ defines an element (which by abuse of notation we call) $\phi \in G(\mathbb{R})$. Now note that if $\gamma \in \mathbb{I}_{\mathbf{C}}$, then $\phi \cdot \rho(\gamma)$ 。 $\psi^{-1}=\rho\left(\phi_{\Pi}(\gamma)\right)$. Thus, by mapping the nontrivial element of $\mathrm{Gal}(\mathbb{C} / \mathbb{R})$ in the crossed product definition of $\mathbb{\Pi}_{\mathbb{R}}$ to $\phi$, we see that we obtain a natural homomorphism

$$
\rho_{\mathbf{R}}: \mathbb{I}_{\mathbb{R}} \rightarrow \mathrm{GL}_{2}^{ \pm}(\mathbb{R})
$$

which extends $\rho_{\mathrm{C}}$ and is such that the composite with the determinant det: 
$\mathrm{GL}_{2}^{ \pm}(\mathbf{R}) \rightarrow \mathbf{R}^{\times}$is trivial on $\mathbb{\Pi}_{\mathbf{C}}$ and equal to the sign representation on $\mathrm{Gal}(\mathbf{C} / \mathbf{R})$. It is this representation $\rho_{\mathbf{R}}$ that will be relevant to our discussion of the $p$-adic case.

\section{$\S 2$. Translation into the $p$-Adic Case}

In this Section, we discuss the dictionary for translating the complex analytic theory of $\S 1$ into the $p$-adic results discussed in $\S 0$. Undoubtedly, the most fundamental tool, which is, in fact, of an algebraic, not an arithmetic nature, is the systematic use of the indigenous bundles of [Gunning]. This enables one to get rid of the upper half plane, and thus to bring uniformization theory into a somewhat more algebraic setting. In any sort of nontrivial arithmetic theory of this nature, however, algebraic manipulations alone can never be enough. Thus, the fundamental arithmetic observation is the following:

Kähler metrics in the complex case correspond to Frobenius actions in the $p^{-a d i c}$ case.

Since one typically gets a natural Frobenius action for free modulo $p$, a Frobenius action typically means a canonical lifting of the natural Frobenius action modulo $p$. In fact, in some sense, if one sorts through the complex analytic theory reviewed $\S 1$, one can essentially distill everything down to two objects. both of which happen to be Kähler metrics:

(1) the hyperbolic metric on a hyperbolic Riemann surface (which encodes the upper half plane uniformization); and

(2) the Weil-Petersson metric on the moduli space (which encodes the Bers uniformization).

Moreover, these two metrics are related to each other in the sense that the latter is essentially the push-forward of the former. In a similar way, the $p$-adic theory revolves around two fundamental Frobenius liftings:

(1) the canonical Frobenius lifting on a canonical hyperbolic curve; and

(2) the canonical Frobenius lifting on a certain stack which is étale over the moduli stack.

The goal of this Section is to explain this analogy in greater detail. 


\section{Gunning's Theory of Indigenous Bundles}

Let $X$ be a compact hyperbolic Riemann surface. Let $H \rightarrow X$ be its uniformization by the upper half plane. Then by considering the covering transformations of $H \rightarrow X$, we get a homomorphism (unique up to conjugation)

$$
\rho: \pi_{1}(X) \rightarrow \operatorname{Aut}(H) \subseteq \mathrm{PSL}_{2}(\mathbb{R})
$$

which we call the canonical representation of $X$. If we regard $\rho$ as defining a morphism into $\mathrm{PSL}_{2}(\mathbb{C})$, then we obtain (in the usual fashion), a local system of $\mathbb{P}^{1}$-bundles on $X$, which thus gives us a holomorphic $\mathbb{P}^{1}$-bundle with connection $\left(P, \nabla_{P}\right)$ on $X$. By Serre's GAGA, $\left(P, \nabla_{P}\right)$ is necessarily algebraic. It turns out that $P$ is always isomorphic to a certain $\mathbb{P}^{1}$-bundle of jets (which is also entirely algebraic). Thus, the upper half plane uniformization may be thought of as just being a special choice of connection $\nabla_{P}$. A pair "like" $\left(P, \nabla_{P}\right)$ (satisfying certain technical properties dicsussed in Chapter I, §2) is called an indigenous bundle. By working with log structures, one can also define indigenous bundles in a natural way for smooth $X$ with punctures, as well as for nodal $X$.

As emphasized earlier, the point of dealing with indigenous bundles is that they allow one to translate the upper half plane uniformization into the purely algebraic information of a connection on $P$. Of course, how one chooses this particular special connection on $P$ is a very nontrivial arithmetic issue. We shall call the pair $\left(P, \nabla_{P}\right)$ consisting of $P$ equipped with this particular connection the canonical indigenous bundle on $X$. Universally, over the moduli stack $\bar{M}_{g, r}$ (of stable $r$-pointed curves of genus $g$ over $\mathbb{C}$ ), the space of all indigenous bundles forms a holomorphic torsor

$$
\bar{\varnothing}_{g, r} \rightarrow \bar{M}_{g, r}
$$

over the logarithmic cotangent bundle $\Omega_{\overline{\mathcal{M}}_{g}, / \mathrm{C}}^{\log }$ of $\bar{M}_{g, r}$. In the holomorphic category, we shall see (in Chapter I, §3) that this torsor is highly nontrivial. In the real analytic category, however, the canonical indigenous bundle determines a trivializing section

$$
s_{H}: \bar{\varnothing}_{g, r} \rightarrow \bar{M}_{g, r}
$$

of this torsor.

In fact, indigenous bundles also allow us to translate such differentialgeometric information as the hyperbolic geometry of $X$ into algebraic terms. For instance, consider the degeneration of Riemann surfaces from the point of 
view of hyperbolic geometry. As reviewed in $\S 1$, this may be thought of in terms of certain geodesic partition curves whose lengths go to zero as a family of smooth $X_{t}$ degenerates to a nodal Riemann surface $Z$. From the complex theory, we know that the hyperbolic metric on $X_{t}$ degenerates to the hyperbolic metric on $Z$. Using indigenous bundles, we can translate this into a more algebraic statement as follows: We define the canonical indigenous bundle on $Z$ to be the indigenous bundle obtained by gluing together the canonical indigenous bundles of the pointed Riemann surfaces occurring in the normalization of $Z$. Then the statement is that as $X_{t}$ degenerates to $Z$, the canonical indigenous bundle on $X_{t}$ degenerates to the canonical indigenous bundle on $Z$. The statement that the lengths of the partition geodesics go to zero then takes the form that the monodromy of the limit indigenous bundle of the canonical indigenous bundles of the $X_{t}$ 's is nilpotent at the nodes.

\section{The Canonical Coordinates Associated to a Kähler Metric}

In this subsection we discuss how a Kähler metric on a complex manifold can be used to define canonical affine, holomorphic coordinates on the manifold locally in a neighborhood of a given point. We believe that what is discussed here is well-known, but our point of view is somewhat different from that usually taken in the literature.

Let $M$ be a smooth complex manifold of complex dimension $m$. The com plex analytic structure on $M$ defines, in particular, a real analytic structure on $M$. Let $\mu$ be a real analytic $(1,1)$-form on $M$ that defines a Kähler metric in $M$. In particular, $\mu$ is a closed differential form. Let $M^{\mathrm{c}}$ be the conjugate complex manifold to $M$ : that is to say, we take $M^{c}$ to be that complex manifold which has the same underlying real analytic manifold structure as $M$, but whose holomor phic functions are the anti-holomorphic functions of $M$. Let us fix a point $e \in$ $M$. Let $N$ be the germ of a complex manifold obtained by localizing the complex manifold $M^{\mathrm{c}} \times M$ at $(e, e) \in M^{\mathrm{c}} \times M$ (where this last expression makes sense since $M^{\mathrm{c}}$ has the same underlying set as $M$ ). Let $\Omega^{\text {hol }}$ (respectively, $\Omega^{\text {ant }}$ ) be the holomorphic vector bundle on $N$ obtained by pulling back the bundle $\Omega_{M}$ (respectively, $\Omega_{M^{c}}$ ) of holomorphic differentials on $M$ (respectively, $M^{\mathrm{c}}$ ) to $M^{\mathrm{c}} \times$ $M$ via the projection $M^{\mathrm{c}} \times M \rightarrow M$ (respectively, $M^{\mathrm{c}} \times M \rightarrow M^{\mathrm{c}}$ ), and then restricting to $N$. Thus, in summary, we have a $2 m$-dimensional germ of a complex manifold $N$, together with two $m$-dimensional holomorphic vector bundles (locally free sheaves) $\Omega^{\text {hol }}$ and $\Omega^{\text {ant }}$ on $N$.

Note that locally at $e \in M$, the fact that $\mu$ is real analytic means that we can write $\mu$ as a convergent power series in holomorphic and anti-holomorphic local coordinates at $e$. In other words, if we restrict $\mu$ to $N$, we may regard $\left.\mu\right|_{N}$ 
as defining a holomorphic section of $\Omega^{\text {hol }} \otimes_{\mathscr{O}_{N}} \Omega^{\text {ant }}$ (where $\mathscr{O}_{N}$ is the sheaf of holomorphic functions on $N$ ). Let $d^{\text {hol }}$ (respectively, $d^{\text {ant }}$ ) be the exterior derivative on $N$ with respect to the variables coming from $M$ (respectively, $M^{\mathrm{c}}$ ). Note that since $\Omega^{\text {hol }}$ is constructed via pull-back from $M$, we can apply $d^{\text {ant }}$ to sections of $\Omega^{\text {hol }}$. We thus obtain a sort of de Rham complex with respect to $d^{\text {ant. }}$

$$
0 \longrightarrow \Omega^{\text {hol }} \stackrel{d^{\text {ant }}}{\longrightarrow} \Omega^{\text {hol }} \otimes_{\mathscr{O}_{N}} \Omega^{\text {ant }} \stackrel{d^{\text {ant }}}{\longrightarrow} \Omega^{\text {hol }} \otimes \mathscr{O}_{N}\left(\wedge^{2} \Omega^{\text {ant }}\right) \stackrel{d^{\text {ant }}}{\longrightarrow} \cdots
$$

Relative to this complex, the section $\left.\mu\right|_{N}$ of $\Omega^{\text {hol }} \otimes \Omega^{\text {ant }}$ satisfies $\left.d^{\text {ant }} \mu\right|_{N}=0$ (since $\mu$ is a closed form). It thus follows from the Poincare Lemma that there exists a (holomorphic) section $\alpha$ of $\Omega^{\text {hol }}$ that vanishes at $(e, e) \in N$ and satisfies $d^{\text {ant }} \alpha=$ $\left.\mu\right|_{N}$. Let $M_{e}$ be the germ of a complex manifold obtained by localizing $M$ at $e \in$ $M$. Let

$$
\iota: M_{e}^{c} \hookrightarrow N
$$

be the inclusion induced by the map $M^{\mathrm{c}} \rightarrow M^{\mathrm{c}} \times M$ that takes $f \in M^{\mathrm{c}}$ to $(f, e) \in M^{\mathrm{c}}$ $\times M$. Then $\iota^{*}(\alpha)$ defines a holomorphic morphism $\beta: M_{e}^{c} \rightarrow \Omega_{M, e}$, where $\Omega_{M, e}$ is the affine complex analytic space defined by the cotangent space of $M$ at $e$. Note, moreover, that although $\alpha$ (as chosen above) is not unique, $\beta$ is nonetheless independent of the choice of $\alpha$. Moreover, $\beta$ is an immersion: Indeed, to see this. it suffices to check that the map induced by $\beta$ on tangent spaces is an isomorphism, but this follows from the fact that $d^{\text {ant }} \alpha=\left.\mu\right|_{N}$, and the fact that the Hermitian form defined by $\mu$ is nondegenerate.

In summary, we see that from the Kähler metric $\mu$, we obtain a canonical holomorphic local affine uniformization

$$
\beta^{\mathrm{c}}: M_{e} \hookrightarrow \Omega_{M, e}^{\mathrm{c}}
$$

Pulling back the standard affine coordinates on $\Omega_{M, e}^{c}$ gives us a canonical collection of holomorphic coordinates on $M_{e}$.

Definition 2.1. We shall refer to these coordinates as the canonical holomorphic local coordinates of the Kähler manifold $(M, \mu)$ at $e$. We shall refer to $\beta^{c}$ as the canonical local affine uniformization of the Kähler manifold $(M, \mu)$ at $e$.

Now let us consider some basic well-known examples: 
Example 1. Let $M=\{z \in \mathbb{C}|| z \mid<1\}$, with the standard hyperbolic metric $\frac{2 d z \wedge d \bar{z}}{\left(1-|z|^{2}\right)^{2}}$. Then $z$ is a canonical coordinate at 0 . Indeed, to see this it suffices to note that $d^{\text {hol }}(z \cdot d \bar{z})=d z \wedge d \bar{z}$, which is equal to the metric modulo the ideal generated by $\bar{z}$ in $\mathscr{O}_{N}$. Note that by the Köbe uniformization theorem, this example essentially covers all hyperbolic Riemann surfaces.

Example 2. Let $M$ be the Teichmüller space of Riemann surfaces of genus $g$ with $r$ punctures, where $2 g-2+r \geq 1$. Then as stated earlier, it is known ([Royd]) that the coordinates arising from the Bers embedding are canonical coordinates with respect to the Weil-Petersson metric on $M$. In fact, in this case, by Theorem 2.3 (proven below) the real analytic section $s_{H}$ defined by the canonical indigenous bundle essentially already serves as an " $\alpha$ " in the above discussion. Thus, in a very real sense, the section $s_{H}$ already is the Bers embed. ding.

\section{The Weil-Petersson Metric from the Point of View of Indigenous Bundles}

Let $X$ be a compact hyperbolic Riemann surface. Let $\left(\pi: P \rightarrow X, \nabla_{P}\right)$ be the canonical indigenous bundle on $X$. Let $\operatorname{Ad}(P)=\pi_{*} \tau_{P / X}$ be the push-forward of the relative tangent bundle of $\pi$. Thus, $\operatorname{Ad}(P)$ is a rank 3 vector bundle on $X$, equipped with a simple Lie algebra structure, hence with a nondegenerate Killing form $\left\langle-,->\right.$ : Ad $(P) \bigotimes_{\mathcal{B}_{r}} \operatorname{Ad}(P) \rightarrow \mathscr{O}_{X}$. Moreover. $\nabla_{P}$ induces a connection $\nabla_{\mathrm{Ad}}$ on $\operatorname{Ad}(P)$. Moreover, as an indigenous bundle, $\operatorname{Ad}(P)$ comes equipped with a section $\sigma: X \rightarrow P$ (the "Hodge section") which defines a Hodge filtration $F^{\bullet}(\operatorname{Ad}(P))$ on $\operatorname{Ad}(P)$. (See Chapter I for more details.) At any rate, we can take the first de Rham cohomology $H_{\mathrm{DR}}^{1}\left(\operatorname{Ad}(P), \nabla_{\mathrm{Ad}}\right)$ module of $\left(\operatorname{Ad}(P), \nabla_{\mathrm{Ad}}\right)$. The Hodge filtration on $\operatorname{Ad}(P)$ then defines a Hodge filtration on the de Rham cohomology, hence an exact sequence:

$$
0 \rightarrow H^{0}\left(X, \omega_{X}^{\otimes 2}\right) \rightarrow H_{\mathrm{DR}}^{1}\left(\operatorname{Ad}(P), \nabla_{\mathrm{Ad}}\right) \rightarrow H^{1}\left(X, \tau_{X}\right) \rightarrow 0
$$

On the other hand, recall the representation that we used to define $\left(P, \nabla_{p}\right)$ :

$$
\rho: \pi_{1}(X) \rightarrow \operatorname{Aut}(H) \subseteq \mathrm{PSL}_{2}(\mathbb{R})
$$

Let $\operatorname{Ad}\left(V_{\mathbf{R}}\right)$ denote the $\pi_{1}(X)$-module obtained by letting $\pi_{1}(X)$ act on the Lie algebra $s l_{2}(\mathbb{R})$ by applying $\rho$ and then conjugating matrices. Let $\operatorname{Ad}\left(V_{\mathbf{C}}\right) \stackrel{\text { def }}{=}$ $\operatorname{Ad}\left(V_{\mathbf{R}}\right) \otimes_{\mathbf{R}} \mathbf{C}$. Then (it is elementary that) we have a "comparison theorem" that gives a natural isomorphism between the de Rham cohomology module just considered and the group cohomology of $\operatorname{Ad}\left(V_{\mathbf{C}}\right)$ : 


$$
H_{\mathrm{DR}}^{1}\left(\operatorname{Ad}(P), \nabla_{\mathrm{Ad}}\right) \cong H^{1}\left(\pi_{1}(X), \operatorname{Ad}\left(V_{\mathrm{C}}\right)\right)
$$

On the other hand, we also have:

$$
H^{1}\left(\pi_{1}(X), \operatorname{Ad}\left(V_{\mathbf{C}}\right)\right) \cong H^{1}\left(\pi_{1}(X), \operatorname{Ad}\left(V_{\mathbf{R}}\right)\right) \bigotimes_{\mathbb{R}} \mathbb{C}
$$

which, combined with the above comparison theorem, thus gives a real structure on $H_{\mathrm{DR}}^{1}\left(\operatorname{Ad}(P), \nabla_{\mathrm{Ad}}\right)$. One way to express this real structure is as an $\mathbb{R}$-linear conjugation morphism (read: "Frobenius action") $c_{\mathrm{DR}}: H_{\mathrm{DR}}^{1}\left(\operatorname{Ad}(P), \nabla_{\mathrm{Ad}}\right) \rightarrow$ $H_{\mathrm{DR}}^{1}\left(\operatorname{Ad}(P), \nabla_{\mathrm{Ad}}\right)$.

Now let us consider the relationship between $c_{\mathrm{DR}}$ and the Hodge filtration. If we compose the natural inclusion $H^{0}\left(X, \omega_{X}^{\otimes 2}\right) \hookrightarrow H_{\mathrm{DR}}^{1}\left(\mathrm{Ad}(P), \nabla_{\mathrm{Ad}}\right)$ with $c_{\mathrm{DR}}$ followed by the natural projection $H_{\mathrm{DR}}^{1}\left(\operatorname{Ad}(P), \nabla_{\mathrm{Ad}}\right) \rightarrow H^{1}\left(X, \tau_{X}\right)$, we obtain a $\mathbb{C}$-bilinear form

$$
\beta: H^{0}\left(X, \omega^{\otimes 2}\right) \otimes_{\mathbb{C}} H^{0}\left(X, \omega^{\otimes 2}\right)^{\mathrm{c}} \rightarrow \mathbb{C}
$$

(where the superscript " $c$ " stands for the complex conjugate $\mathbb{C}$-vector space).

Proposition 2.2. The form $\beta$ is precisely the Weil-Petersson metric on quadratic differentials defined in $\S 1$ by means of integration. In particular. $\beta$ is nondegenerate.

Proof. In order to obtain $\beta$, we implicitly used the special case of Serre duality given by $H^{1}\left(X, \tau_{X}\right) \cong H^{0}\left(X, \omega_{X}^{\otimes 2}\right) \vee$. But in the complex analytic context, the pairing that defines this sort of duality is given by integrating the product of $((0,1)-$ and $(1,0)-)$ forms. The volume form $v_{X}$ appears for the sake of defining the duality between $\omega_{X}$ and $\bar{\omega}_{X}$. With these remarks, the claim of the Lemma becomes a tautology.

Now let us recall the real analytic section $s_{H}: \bar{M}_{g, r} \rightarrow \overline{\mathscr{\phi}}_{g, r}$. Since $\overline{\mathscr{\otimes}}_{g, r} \rightarrow$ $\overline{\mathcal{M}}_{g . r}$ is a holomorphic torsor, we may form $\bar{\partial} s_{H}$, which gives a section of $\Omega_{\bar{M}_{r} / \mathrm{C}}^{\log } \otimes$ $\bar{\Omega}_{\overline{.1}_{q}, / \mathrm{C} \text {. }}^{\operatorname{lng}}$ On the other hand, the Weil-Petersson metric also defines a section $\mu_{\mathrm{WP}}$ of $\Omega_{\bar{M}_{g} / \mathrm{C}}^{\log } \otimes \bar{\Omega}_{\bar{M}_{g} / \mathrm{C} \text {. Now we have the following result (stated in }}^{\log }$ [ZT], but from a somewhat different point of view):

Theorem 2.3. The form $\bar{\partial} s_{H}$ is equal to $\mu_{\mathrm{WP}}$.

Proof. By introducing log structures. one can handle the general case; here, 
for simplicity, we restrict our attention to the case of smooth compact Riemann surfaces. Let us consider the composite of the natural inclusion $H^{0}\left(X, \omega_{X}^{\otimes 2}\right) \hookrightarrow$ $H_{\mathrm{DR}}^{1}\left(\operatorname{Ad}(P), \nabla_{\mathrm{Ad}}\right)$ with $c_{\mathrm{DR}}$ followed by the natural projection $H_{\mathrm{DR}}^{1}\left(\mathrm{Ad}(P), \nabla_{\mathrm{Ad}}\right)$ $\rightarrow H^{1}\left(X, \tau_{X}\right)$; this composite gives a $\mathbb{C}$-linear morphism:

$$
H^{0}\left(X, \omega_{X}^{\otimes 2}\right) \rightarrow H^{1}\left(X, \tau_{X}\right)^{\mathrm{c}}
$$

which is invertible by Lemma 2.2. Taking its inverse, and dualizing, we obtain an element

$$
\delta \in H^{0}\left(X, \omega_{X}^{\otimes 2}\right) \otimes_{\mathrm{C}} H^{0}\left(X, \omega_{X}^{\otimes 2}\right)^{\mathrm{c}}
$$

On the other hand, sorting through the definitions, it is a tautology in linear algebra that the value of $\bar{\partial} s_{H}$ at the point $[X] \in M_{g}$ is given by $\delta$. But, combining this with Lemma 2.2 , we see that we have proven the Theorem.

The important point here is that this Theorem shows that:

The Weil-Petersson metric, and hence the Bers embedding, is obtained precisely by considering the extent to which "Frobenius" - i.e., complex conjugation - is compatible with the canonical indigenons bundle section $s_{H}$.

Stated in this way, the classical complex theory becomes all the more formally analogous to the $p$-adic theory to be discussed in this paper.

\section{The $\mathbb{P h i l o s o p h y ~ o f ~} \mathbb{K a ̈ h l e r} \mathbb{M}$ etrics as Frobenius Liftings}

Before going into a detailed account of the correspondence between complex and $p$-adic results, we pause to explain some of the motivation for considering Kähler metrics as Frobenius liftings. Let $S$ be a smooth $p$-adic formal scheme over $\mathbb{Z}_{p}$. A Frobenius lifting on $S$ is a morphism $\Phi: S \rightarrow S$ whose reduction modulo $p$ is equal to the Frobenius morphism in characteristic $p$. Then the main point of the analogy is that just as (real analytic) Kähler metrics define canonical coordinates (as discussed above). Frobenius liftings $\Phi: S \rightarrow S$ (that satisfy a certain technical condition called ordinariness - see Chapter III, $\$ 1$ for details) also define canonical coordinates, as follows:

The most basic example of an ordinary Frobenius lifting is the case when $S$

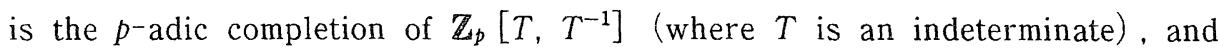
$\Phi^{-1}(T)=T^{p}$. Then the theory of ordinary Frobenius liftings (Chapter III, $\S 1)$ states that by means of a certain "integration" procedure, every ordinary 
Frobenius lifting on an arbitrary $S$ becomes (after completing at a point of $S$ ) isomorphic to a product of copies of this basic example. This "integration procedure" is thus analogous to the integration procedure just reviewed which allowed us to construct canonical coordinates associated to real analytic Kähler metrics.

\section{The Dictionary}

The fundamental "nuts and bolts" of the complex theory lies in the Beltrami equation. Suppose that we think of the Beltrami equation not as a differential equation whose unknown is the quasiconformal function $f_{\mu}$. but instead as an equation whose unknown is the conformal quasidisk embedding function $\left.f^{\mu}\right|_{H}$ (in the discussion of quasidisks). A quasidisk embedding of the universal covering space of a hyperbolic Riemann surface $X$ defines an indigenous bundle $(P$, $\left.\nabla_{P}\right)_{\mu}$ on $X$ in a natural way. Thus, from this point of view, we can think of the Beltrami equation as an equation whose unknown is $\left(P, \nabla_{P}\right)_{\mu}$. Moreover, the Beltrami coefficient $\mu$ defines the "shearing" or distortion factor between $\bar{z}$ and $z$. Thus, in summary, we may regard the Beltrami equation as an equation in the unknown $\left(P, \nabla_{P}\right)_{\mu}$ in terms of the distortion factor (effected by the quasidisk embedding $\left.f^{\mu}\right|_{H}$ ) between $z$ and its "Frobenius conjugate" $\bar{z}$.

On the other hand, the "nuts and bolts" of the p-adic theory lies in the study of the Verschiebung on indigenous bundles, which occupies most of Chapter II. As a function on the indigenous bundles of a hyperbolic curve in characteristic $p$, the Verschiebung - which is essentially the determinant of the $p$-curvature - measures the distortion factor between applying Frobenius to an infinitesimal on the curve and applying Frobenius to an infinitesimal motion in the ("quasidisk") uniformization defined by the indigenous bundle. Thus, for instance, when the $p$-curvature is nilpotent, there is no distortion factor, and so the indigenous bundle provides the "right" uniformization for the curve. In this sense, we feel that there is an analogy between the Beltrami equation in the complex theory and the Verschiebung on indigenous bundles in the p-adic theory.

Relative to this analogy, the fundamental existence and uniqueness theorem for solutions to the Beltrami equation becomes the result (in Chapter II) that the Verschiebung on indigenous bundles is finite and flat. Since in the p-adic case, its degree is not one, we only have uniqueness up to a finite number of possibilities. This is why we get several distinct "quasiconformal equivalence classes" in the $p$-adic case. Moreover, the important integral operator " $T$ "-i.e., the parametrix to $\bar{\partial}$ - which gives the first term in the series expansion for $f_{\mu}$ may be regarded as having its analogue in the $p$-adic theory in the infinitesimal 
Verschiebung, which plays an important role throughout the paper.

More obvious is the analogy between the canonical representation $\rho_{\mathbf{c}}$ : $\pi_{1}(X) \rightarrow \mathrm{PSL}_{2}(\mathbf{R})$ of a hyperbolic Riemann surface (arising from the upper half plane uniformization), and the canonical representation $\rho_{\infty}: \Pi_{\infty} \rightarrow \mathrm{GL}_{2}^{ \pm}\left(\mathbf{Z}_{p}\right)$ of an ordinary $p$-adic curve (in Theorem 0.4 ). Of course in the $p$-adic case, $\Pi_{\infty}$ has a substantial arithmetic part in addition to its geometric part. Although generally in the complex case, there is not much of a Galois group to work with, at least for real curves, we saw at the end of $\S 1$, that one does get a natural representation $\rho_{\mathbf{R}}$ of the full "arithmetic fundamental group" $\Pi_{\mathbf{R}}$ into $\mathrm{GL}_{2}^{ \pm}(\mathbf{R})$. Moreover, our approach to constructing $\rho_{\infty}$ in the $p$-adic case is very much akin to Bers' approach to constructing $\rho_{\mathrm{C}}$ in the complex case: Namely, if one traces through the proof (which lies in Chapters II through V), one sees that effectively what we are doing is noting that the result is true for totally degenerate curves, and then transporting this result over the rest of the moduli stack of ordinary curves.

Next let us consider metrics and geometry. As we stated earlier, in some sense, one can summarize the entire complex theory by saying: We start with the hyperbolic (Kähler) metric on a hyperbolic curve, define the WeilPetersson (Kähler) metric on the moduli stack precisely so as to be compatible with the hyperbolic metric on the curves being parametrized; then our holomorphic uniformizations - i.e., both the upper half plane uniformization of the hyperbolic curve and the Bers uniformization of the moduli stack - are obtained by "integrating" the respective metrics. Similarly, the fundamental result in the $p$-adic theory - namely, Theorem 0.1 - is a result about the existence of certain Frobenius liftings on the universal hyperbolic curve and its moduli stack which are uniquely characterized by the fact that they are compatible with each other. Here the compatibility is expressed through the tool of the canonical indigenous bundle. Then, by "integrating" these Frobenius actions, we obtain canonical ( $p$-adically holomorphic) coordinates (as in Corollary 0.2 ) on $\mathscr{C}^{\text {ord }}$ and $\overline{\mathcal{N}}_{g . r}^{\text {ord }}$. This particular analogy lies at the heart of this work.

The Bers coordinates and the coordinates of Chapter III, Theorem 2.4, are appropriate in the locus $\mathcal{M}_{g, r}$ of smooth curves. For totally degenerate curves, one has multiplicative parameters (Chapter III, Definition 2.7) which we believe are analogous to the holomorphic coordinates of degeneration of [Wolp], reviewed in $\S 1$. For instance, both sets of parameters are holomorphic and naturally indexed by the nodes of the totally degenerate curve.

For elliptic curves - regarded parabolically - one has, on the one hand, the 
well-known theory of the hyperbolic upper half plane, or unit disk, in the complex case, and Serre Tate theory in the $p$-adic case. It is interesting to note that at both types of primes (complex and $p$-adic), the parabolic theory may be obtained in a very precise sense as the parabolic specializations, respectively, of Bers' theory and of the hyperbolic $p$-adic theory developed in this paper. In fact, this is one of our reasons for feeling that the canonical $p$-adic coordinates of Corollary 0.2 are the p-adic analogue, not of Teichmiiller's coordinates, but of Bers': Namely, in addition to the fact that Teichmüller's coordinates are not holomorphic, whereas Bers' are, Teichmüller obtains the same coordinates for 1 -pointed curves of genus 1 and parabolic elliptic curves. On the other hand, it is well-known that Bers' coordinates are very different for 1-pointed curves of genus 1 and parabolic elliptic curves, which is consistent with the fact that the canonical coordinates of Corollary 0.2 are also very different for 1-pointed curves of genus 1 and parabolic elliptic curves.

\section{Loose Ends}

We close by saving that although, as described above, there are (what the author believes to be) very strong analogies between Bers' complex theory and the $p$-adic theory presented here, the picture is by no means complete. For instance, one fundamental fact in the complex case is that all $r$-pointed smooth curves of genus $g$ are quasiconformally equivalent, whereas in the $p$-adic case, the theory behaves as though there are several different quasiconformal equivalence classes that are permuted around to each other by a certain monodromy action in such a way that there seems to be no one quasiconformal equivalence class "which is better than the others." Ideally, one would like to have a much more complete understanding of this phenomenon. In particular, one would like to know precisely how many quasiconformal equivalence classes there are (at least generically), as well as a more explicit description of the set of such classes.

Also, I still do not understand what the complex, or global, analogue of a "canonical $p$-adic curve" is. For ordinary elliptic curves, since Serre-Tate canonical liftings have complex multiplication, one can ask what the hyperbolic analogue of having complex multiplication is. Since having complex multiplication for an elliptic curve means having lots of isogenies. it is natural to ask if the proper hyperbolic analogue is having lots of correspondences, which are a sort of higher genus version of isogenies. If a hyperbolic curve does have a lot of correspondences, then one knows ([Marg]) that the image of its canonical representation is arithmetic. In Chapter IV, we prove that a canonical curve has lots of "pseudo-correspondences." but unfortunately, at the time of writing, I do not see how to make these pseudo-correspondences into genuine correspondences, so that one could apply Margulis' result. Another issue that arises in 
this connection is the question of whether one can characterize "hyperbolic curves with complex multiplication" - whatever the correct definition should be for this term - in terms of the Bers coordinates.

Finally, as the title implies, the present work deals exclusively with the case of ordinary curves. In a complete theory, one would like to know what happens when one has a nilpotent indigenous bundle which is not ordinary.

At any rate, in summary, with respect to these three issues of quasiconformal equivalence classes, canonical curves, and non-ordinary curves, much work remains to be done. We hope to be able to address these issues in future papers.

\section{Chapter I: Crystalline Projective Structures}

\section{\$0. Introduction}

The purpose of this Chapter is to study the algebraic analogue of projective structures on a Riemann surface. In particular, we prove many of the analogues of results of [Gunning] in a purely algebraic framework, often making use of the crystalline site where complex analytically one would restrict to a simply connected neighborhood on which one can integrate. Unlike Gunning, we make systematic use of the log structures of [Kato], which enable us to work with a very general sort of "log-curve", that is, we can handle the case of curves with marked points, as well as singular nodal curves on an equal footing to the smooth case.

In $\S 1$, we discuss the notion of a Schwarz structure, which is the algebraic analogue of [Gunning] 's projective structures. We relate Schwarz structures to projective bundles with connections as well as to square differentials, and we show that Schwarz structures naturally give rise to a Schwarzian derivative. (Moreover, in the Appendix to this Chapter, we show that for $\mathbb{P}^{1}$, this abstract notion of a Schwarzian derivative essentially coincides with the classical Schwarzian derivative.) The characterizing feature of $\S 1$ is that everything takes place locally on the curve in question. In $\S 2$, we discuss indigenous bundles (the direct algebraic analogue of [Gunning]'s indigenous bundles). What distinguishes $\S 2$ from $\S 1$ is that in $\S 2$, we work mainly over stable curves, and thus global issues on the curve come into play. In $\S 2$, we are still working locally, however, on the base. In $\S 3$, we perform various intersection theory 
calculations that allow us to prove that in most cases, there do exist any canonical indigenous bundles on the universal smooth curve over a moduli stack. Thus, in $\S 3$, we are concerned with issues that are global not only on the curve, but also on the base. It should be said that all the material in this Chapter is, in some sense, "well-known," but I do not know of any modern reference that does things from this point of view. In particular, all the references that I know of (with the exception of [Ih], which is algebraic, but somewhat different in point of view) discuss things only in the complex analytic case, and often work with " $h_{\alpha \beta}$ 's" (i.e., cocycle classes) rather than with objects that have an intrinsic meaning.

\section{§1. Schwarz Structures}

In this Section, we introduce the crystalline analogue of what Gunning calls "projective structures on a Riemann surface." (We shall call them Schwarz structures (after the Schwarzian derivative) to distinguish them from the analytic notion.) We begin by letting $S$ be a connected noetherian scheme. Often, we shall prove results about arbitrary stable curves by working on various compactified moduli stacks. Thus, even if one is ultimately interested only in smooth curves, for certain proofs, we shall see that it is useful to develop the machinery for arbitrary stable curves. To deal with singular curves, we shall use the theory of log schemes of [Kato]. Thus, we assume that $S$ has a given fine ([Kato], §2) log structure, and denote the resulting log scheme by $S^{\log }$.

\section{Notation and Basic Definitions}

Definition 1.1. Let $f^{\log }$ : $U^{\log } \rightarrow S^{\log }$ be a morphism of log schemes whose underlying morphism of schemes $f: U \rightarrow S$ is of finite type, flat and of relative dimension one. Then we shall say that $f^{\mathrm{log}}$ is locally stable of dimension onc if, for every point $u \in U$, there exist étale morphisms $T \rightarrow S$ and $V \rightarrow U \times s T$. together with $v \in V^{r}$ mapping to $u \in U$ such that when we pull-back the log structure on $S$ (respectively, $U$ ) to $T$ (respectively, $V$ ) to obtain $\log$ schemes $T^{\log }$ and $V^{\mathrm{log}}$, one of the following holds:

(1) $V \rightarrow T$ is smooth, and $V^{\log }=V \times{ }_{T} T^{\log }$ (where $V$ and $T$ denote the $\log$ schemes with trivial $\log$ structure); or

(2) $V \rightarrow T$ is smooth, and there exists a section $s: T \rightarrow V$ such that if we denote by $V^{s}$ the $\log$ scheme defined by the relative divisor $\operatorname{Im}(s)$ on $V$, then $V^{\log }=V^{s} \times{ }_{T} T^{\log }$; or 
(3) let $Y=\operatorname{Spec}(\mathbb{Z}[t]) ; X=Y[x, y] /(x y-t)$ (where $x, y$, and $t$ are indeterminates) and endow $Y$ (respectively, $X$ ) with the $\log$ structure arising from the divisor $t=0$ (respectively, $x y=0$ ), so we get a morphism $X^{\log } \rightarrow Y^{\log }$ of $\log$ schemes; then there exists a morphism of $\log$ schemes $T^{\log } \rightarrow Y^{\log }$, together with a morphism $\zeta^{\log }: V^{\log } \rightarrow T^{\log } \times{ }_{\gamma^{\log }} X^{\log }$ such that the underlying scheme morphism $\zeta$ of $\zeta^{\log }$ is étale, and the $\log$ structure of $V^{\log }$ on $V$ is the pull-back via $\zeta$ of the $\log$ structure on $T^{\log } \times_{Y^{108}} X^{\mathrm{log}}$.

In case (1) (respectively, (2); (3)), we shall say that $f$ is smooth and unmarked (respectively, marked; singular) at $u$.

Note that if $f^{\log }: U^{\log } \rightarrow S^{\log }$ is locally stable of dimension one, then it is always $\log$ smooth ([Kato], §3). Also, note that by étale descent, the images in $U$ of all the sections $s$ as in Case (2) above form a divisor in $U$ which is étale over $S$. We shall refer to this divisor as the divisor of marked points in $U$.

Now let us suppose that there exists an odd prime $p$ which is nilpotent on $S$. We also suppose that we are given a closed subscheme $S_{0}=V(\mathscr{L}) \subseteq S$, where the sheaf of ideals $\mathscr{J}$ has a divided power structure $\gamma$. We denote the $\log$ scheme $S_{0} \times{ }_{S} S^{\log }$ (where $S_{0}$ and $S$ denote the log schemes which are the respective schemes endowed with the trivial $\log$ structure) by $S_{0}^{\log }$. Let $f^{\text {log. }} U^{\text {log }}$ $\rightarrow S^{\log }$ be locally stable of dimension one. Then we shall call a section of $D_{\Delta}$ $\left(U^{\log } \times S^{\log } U^{\log }\right)$ (the PD-envelope of the diagonal, as in [Kato], §5) a bianalytic function over $U^{10 g}$. Note that the bianalytic functions form a sheaf, which we denote $\mathscr{O}_{u^{b}}$, on the étale site of $U$. Let $\mathscr{O}_{u}$ denote the sheaf on the étale site of $U$ given by considering ordinary functions. Then the two projections $U \times{ }_{S} U \rightarrow U$

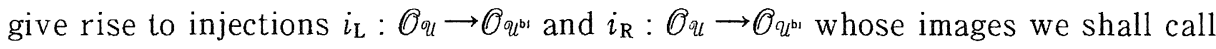
the left-sided (respectively, right-sided) bianalytic functions on $U^{10 g}$. We shall also refer to right-sided bianalytic functions as constant bianalytic functions, or bianalytic constants. We denote tensor products of an $\mathbb{O}_{\boldsymbol{u}}$-module $\mathscr{F}$ over $\mathcal{O}_{\boldsymbol{u}}$ with $\mathscr{O}_{q^{\prime \prime}}$ via $i_{\mathrm{L}}$ (respectively, $i_{\mathrm{R}}$ ) by writing $\mathscr{F}$ on the left (respectively, right). Finally, we have a multiplication morphism $\mu: \mathscr{O}_{q^{b}} \rightarrow \mathbb{O}_{\mathscr{q}}$. We denote the ideal subsheaf of $\mathscr{O}_{\mathscr{U}^{\circ}}$ which is the kernel of $\mu$ by $\mathscr{g}$. We shall say that a bianalytic functions $f$ over some étale $V \rightarrow U$ is a bianalytic uniformizer on $V$ if $f$ is, in fact. a section of $\mathscr{g}$ which generates the line bundle $\mathscr{g} / \mathscr{g}^{[2]} \cong \omega_{/^{108} / S^{108}}$ as an $\mathscr{O}_{\mathcal{U}}$-module. Let $\mathscr{O}_{\hat{q}}{ }^{b}$ be the complection of $\mathscr{O}_{\hat{q}}{ }^{b}$ with respect to the divided powers $\mathscr{g}^{[l]}$, and let $\hat{\mathscr{J}} \subseteq \mathscr{O}_{\hat{u^{b}}}$ the closure of $\mathscr{g}$ in $\mathscr{O}_{\hat{u}^{b .}}$. We shall call sections of $\mathscr{O}_{\hat{u}}$ biformal functions, and use similar terminology for biformal functions as we do for bianalytic functions. 
Occasionally, we shall also need to make use of trianalytic (respectively, triformal) functions, i.e., sections of $D_{\Delta}\left(U^{\log } \times S^{\log } U^{\log } \times{ }_{S^{\log }} U^{\log }\right)$ (respectively, its completion with respect to the divided powers of the diagonal ideal). We denote the sheaf of trianalytic functions (respectively, triformal) on the étale site of $U$ by $\mathscr{O}_{u^{\text {r }}}$ (respectively, $\mathscr{O}_{\widehat{Q}^{t r}}$ ), and we have left, right, and middle injections $j_{1}$,

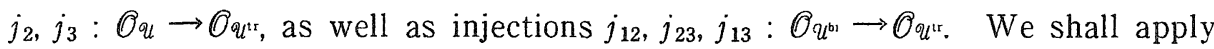
similar terminology and notation to trianalytic or triformal functions to that applied already to bianalytic functions. In particular, we shall call trianalytic functions that are in the image of $j_{23}$ trianalytic constants.

Definition $\mathbb{1}$.2. Let $\& \subseteq \mathscr{O}_{\hat{u}^{b}}$ be a subsheaf in the category of sets. We shall call \& a Schwarz (respectively, pre-Schwarz) structure on $U^{\log }$ if étale locally on $U$ (i.e., for some étale cover $V \rightarrow U$ ), \& has the following form: there exists some biformal uniformizer $z \in \Gamma(V, \mathscr{S})$ such that for every étale $W \rightarrow V$, and every section $f \in \Gamma\left(W, \mathscr{O}_{\widehat{u}}{ }^{b}\right)$, then $f \in \Gamma(W, \mathscr{S})$ if and only if (respectively, implies that) $f$ can be written étale locally (on $W$ ) in the form $(a z+b) /(c z+d)$, where $a, b, c, d$ are biformal constants and $d$ is invertible.

It is clear that if $\mathscr{S} \subseteq \mathcal{O}_{\widehat{u}}$ " is a pre-Schwarz structure on $U^{\log }$, then $\mathscr{\&}$ is contained in a unique Schwarz structure $\mathscr{S}^{a} \subseteq \mathcal{O} \hat{\mathrm{q}}^{\mathrm{b}}$ on $U^{\mathrm{log}}$, which we refer to as the Schwarz structure associated to $\&$. If $\&$ is a Schwarz structure, then we shall denote by $\mathscr{\&}^{\times} \subseteq \&\left(\right.$ respectively, $L_{\&}$ ) the subsheaf consisting locally of functions of the form $(a z+b) /(c z+d)$, where: (1) $z$ is a biformal uniformizer belonging to $\mathscr{S}$; (2) $d$ is invertible; and (3) $\left(\begin{array}{ll}a & b \\ c & d\end{array}\right)$ is an invertible matrix of biformal constants (respectively, $b=0$ ). We let $L_{\diamond}^{\times}=L_{\delta} \cap \mathscr{S}^{\times}$. Thus $\mathscr{S}^{\times}, L_{\lrcorner}$, and $L_{\delta}^{\times}$are all pre-Schwarz structures. We shall call $L_{\&}^{\times}\left(\right.$respectively, $\left.L_{\delta}\right)$ the sheaf of bifor. mal uniformizers (respectively, pseudo-uniformizers) of $\&$.

Let $G \rightarrow U$ be the group scheme $\mathrm{PGL}_{2}$, and let $B \subseteq G$ be the subgroup scheme which is the standard Borel subgroup of $\mathrm{PGL}_{2}$, i.e., the image of the lower triangular matrices.

\section{First Properties of Schwarz Structures}

Propostion $\mathbb{1} .3$. The subsheaf $L_{.3}^{x} \subseteq \&$ consisting of biformal uniformizers of $\&$ forms a B-torsor $B \& \rightarrow U$.

Proof. This follows immediately from the definition of a Schwarz structure. The action of $B$ is given by associating to a biformal uniformizer $z$ and a matrix $\left(\begin{array}{ll}a & 0 \\ c & d\end{array}\right)$ (where $a, c, d$ are biformal constants, and $a, d$ are invertible) the bifor- 
mal uniformizer $a z /(c z+d)$.

Note that every $B$-torsor $T \rightarrow U$ naturally defines a $\mathbf{P}^{1}$-bundle with a given section (by taking the quotient of $\mathbb{P}^{1} \times{ }_{U} T$ modulo the diagonal action of $B$, where $B$ acts on $\mathbf{P}^{1}$ by means of affine transformations that fix zero; the section is the image of the zero section of $\mathbf{P}^{1}$ ). We shall refer to the $\mathbf{P}^{1}$-bundle $P_{\&} \rightarrow U$ associated to $B_{\&} \rightarrow U$ as the $\mathbf{P}^{1}$-bundle associated to the Schwarz structure \&. We denote by $\sigma_{\&}: U \rightarrow P_{\&}$ the natural section (arising from the fact that the structure group is $B$ rather than $G$ ).

Proposition 1.4. Let \& be a Schuarz structure on $U^{1 \log }$. Then $P_{\&} \cong \mathbb{P}$

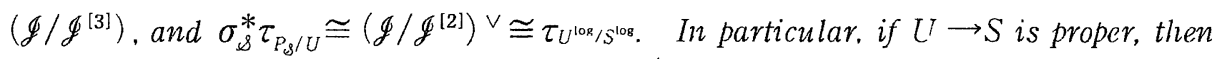
the height of $\sigma_{\&}$ with respect to $\tau_{P_{S} / U}$ is $-\operatorname{deg}\left(\omega_{U^{\text {Iog }} s^{\text {Iog }}}\right)$.

Proof. One sees by construction (e.g., by writing out transition functions) that the sheaf of nonzero relative rational functions of relative degree one (as in [EGA IV], §20) for $P_{s} \rightarrow U$ that vanish at $\sigma$ is naturally isomorphic to $L_{\S}^{\times}$. Thus, by considering Taylor expansions out to second order terms, we get an isomorphism $\mathscr{O}_{P_{S}}\left(-\sigma_{\mathscr{S}}\right) / \mathscr{O}_{P_{S}}\left(-3 \sigma_{\mathscr{S}}\right) \cong \mathscr{J} / \mathscr{g}^{[3]}$ (here we use that $p$ is odd). On the other hand, by multiplying and then taking the residue at $\sigma$, we obtain a

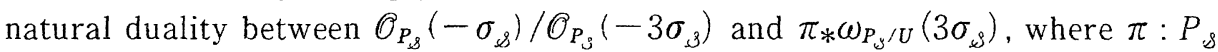
$\rightarrow U$ is the natural projection. Also, note that via this duality, the filtration in-

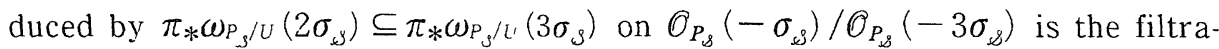
tion defined by the submodule $\mathscr{O}_{P_{s}}\left(-2 \sigma_{\mathscr{S}}\right) / \mathscr{O}_{P_{S}}\left(-3 \sigma_{\mathscr{S}}\right)$. Since $P_{\mathscr{S}}$ is clearly naturally isomorphic to the projectivization of $\pi_{*} \omega_{P_{S} / L^{\prime}}\left(3 \sigma_{\mathscr{S}}\right)$, we thus obtain the result.

\section{Crystalline Schwarz Structures and Monodromy}

Let $\&$ be a Schwarz structure on $U^{\mathrm{log}}$. We would like to associate to \& a subsheaf (in the category of sets) of $\mathscr{O}_{\hat{u}}$ tr. which we shall call $\mathscr{S}_{12}$ as follows. We work locally. Thus, we assume that there exists a biformal uniformizer $z \in$ $\Gamma(U, \&)$. We consider the triformal function $z_{12}$ defined by $j_{12}(z)$, where $j_{12}$ is the natural map $\mathscr{O}_{\hat{\mathcal{u}}} \rightarrow \mathscr{O}_{\hat{\mathcal{U}}^{t r}}$ given by inclusion on the first two factors. Then we let $\oiint_{12}$ be the sheaf of all functions which étale locally can be written in the form $\left(a z_{12}+b\right) /\left(c z_{12}+d\right)$, where $a, b, c, d$ are triformal constants and $d$ is invertible. Note that the definition of $\mathscr{S}_{12}$ does not depend on the choice of $z$, so everything glues together, and we obtain the subsheaf $\mathscr{S}_{12}$ of $\mathscr{O}_{\hat{\mathscr{u}}}{ }^{1 \text {, }}$ over our original $U$. On the other hand, we also have a subsheaf $\mathscr{S}_{13}$ of $\mathscr{O}_{\widehat{U}^{4}}$ defined in the same way as $\oiint_{12}$, except with the roles of 2 and 3 reversed. 
Defimition 1.5 . We shall say that the Schwarz structure $\&$ is a crystalline

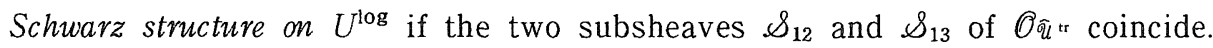
Let $\&$ be a crystalline Schwarz structure on $U^{\text {log }}$. Then we shall say that \& has nilpotent monodromy if for every marked point $s: T \rightarrow V$ (with $V \rightarrow U$ étale), there exists a biformal uniformizer $z \in \mathscr{\&}(V)$ and a section $a \in \omega_{U^{108} / S^{108}}(V)$ such that the image of $(d z)-i_{\mathrm{R}}(a)$ (where " $d$ " is the exterior derivative on the right) in $\mathfrak{O}_{\tilde{q}^{b}} \otimes \mathcal{O}_{U} s^{*} \omega_{U^{\log / S^{\log }}}$ is zero.

Remark. Of course. one may also phrase the definition of a crystalline Schwarz structure as follows. First, note that $\mathscr{O}_{\hat{q}^{\mathrm{u}}}$, together with its right-hand

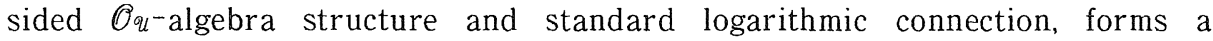
quasi-coherent crystal of algebras $\mathscr{A}$ on the crystalline site of $U^{\log } / S^{\log }$. Then a crystalline Schwarz structure is a subsheaf of the sheaf $\mathscr{A}$ on the crystalline site of $U^{\mathrm{log}} / S^{\log }$ satisfying certain properties. Since this point of view is only formally different from the point of view of Definition 1.5, we shall use these two points of view interchangeably in what follows.

Let us suppose that $\&$ is a Schwarz structure on $U^{\text {log }}$. Let $H \subseteq G$ be the open subscheme consisting of matrices of the form $\left(\begin{array}{ll}a & b \\ c & d\end{array}\right)$, where $d$ is invertible. Note that $H$ is stable under the action by $B$ from the right. Thus, we can take the quotient of $H \times_{U} B . s$ (by the diagonal action of $B$ ) to obtain a fiber bundle $H_{s} \rightarrow U$ with fibers locally isomorphic to $H \rightarrow U$. Similarly, we also obtain a $G$-torsor $G_{33} \rightarrow U$. It now follows immediately from the definitions that the sheaf defined on the étale site of $U$ by $H_{\&}$ is naturally isomorphic to $\mathscr{S}^{\times}$. Thus, if we assume that the Schwarz structure $\&$ is crystalline, we see that we get a natural isomorphism between the two pull-backs of $H_{\mathscr{S}} \rightarrow U$ via $i_{\mathrm{L}} i_{\mathrm{R}}: \mathscr{O}_{\mathscr{U}} \rightarrow \mathscr{O}_{\hat{q}}{ }^{b}$, i.e., we get a logarithmic connection $\nabla_{H_{3}}$ on $H_{3} \rightarrow U$. By basic facts about fiber bundles, this gives a logarithmic connection $\nabla_{\hat{r}_{3}}$ on $G_{\&} \rightarrow U$ and a logarithmic connection $\nabla_{P_{s}}$ on $P_{s} \rightarrow U$, as well. Thus, in summary, to every crystalline Schwarz structure $\mathscr{\&}$, we have associated a natural $\mathbb{P}^{1}$-bundle with section and logarithmic connection $\left(P_{\&} \rightarrow U ; \sigma_{\&}: U \rightarrow P_{\diamond} ; \nabla_{P_{\diamond}}\right)$. Moreover, it follows from the definition of the connection $\nabla_{P_{s}}$ that by differentiating $\sigma_{\&}$ by means of $\nabla_{P_{s}}$ we get an isomorphism $\tau_{U^{108} / S^{108}} \cong \sigma_{\&}^{*} \tau_{P_{s}{ }^{\prime} U^{T}}$, which is called the Kodaira-Spencer morphism. Indeed, to see that this morphism is, indeed, an isomorphism, it suffices to realize that if, locally on $U$, one takes a biformal uniformizer $z$, the difference $j_{12}(z)-j_{13}(z)$ generates $j_{23}(\widehat{\mathscr{g}}) \mathcal{O}_{\widehat{u} u^{t r}}$.

In addition to the fact that the Kodaira-Spencer morphism is an isomorphism, the logarithmic connection $\nabla_{P_{3}}$ has another special property: If $\&$ has nilpotent monodromy, then we can make more explicit the way in which this monod- 
romy acts. Indeed, let us recall from [Kato], $\S 6$, that if $s: S \rightarrow U$ is any marked point, then there exists a unique subsheaf $\mathcal{M}_{S}$ of $\left(\mathscr{O}_{\widehat{U}}{ }^{\circ} \widehat{\otimes}_{\mathscr{C}_{U . S}{ }^{-1}} \mathscr{O}_{S}\right) / \mathscr{O}_{S}$ which is isomorphic to $\mathscr{O}_{S}$ and annihilated by the monodromy operator of the standard logarithmic connection on $\mathscr{O}_{\hat{q}}{ }^{\circ}$. (Locally, this subsheaf is generated by $\log (1-\delta)$, where $\delta=1-\left(\frac{1 \otimes t}{t \otimes 1}\right)$, and $t$ is a local generator of the ideal defining s.) This subsheaf $\mathcal{M}_{s}$ thus defines a section $q_{s}: S \rightarrow \mathbb{P}\left(\mathscr{g} / \mathscr{g}^{! 3]}\right)$ that lies over $s$. Then it follows from these observations, plus Proposition 1.4, that

Proposition 1.6. If \& is a crystalline Schwarz structure on $U^{\log }$ with nilpotent monodromy, then under the isomorphism $P_{\&} \cong \mathbf{P}\left(\mathscr{g} / \mathscr{g}^{[3]}\right)$ of Proposition $1.4, q_{s}$ is fixed by the monodromy action on $P_{\&} \cong \mathbb{P}\left(\mathscr{g} / g^{[3]}\right)$ at $s$.

\section{Correspondence with $\mathbb{P}^{1}$-bundles}

So far, from a crystalline Schwarz structure, we have constructed a $\mathbb{P}^{1}$-bundle with section and logarithmic connection (satisfying certain properties). We can go the other way, as well. Suppose we are given a $\mathbb{P}^{1}$-bundle with section and logarithmic connection $\left(\pi: P \rightarrow U ; \sigma: U \rightarrow P ; \nabla_{P}\right)$ such that the Kodaira-Spencer morphism obtained by differentiating $\sigma$ via $\nabla_{P}$

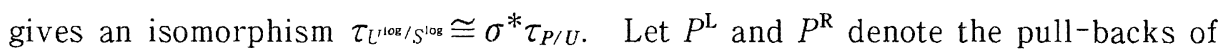
$\pi: P \rightarrow U$ via $i_{\mathrm{L}}, i_{\mathrm{R}}: \mathcal{O}_{\mathcal{U}} \rightarrow \mathscr{O}_{\hat{u}^{b}}$. respectively. Then the connection $\nabla_{P}$ defines an O $\widehat{\hat{U}}^{\mathrm{t}}$-linear isomorphism $\Xi: P^{\mathrm{L}} \cong P^{\mathrm{R}}$. Thus, we have a commutative diagram:

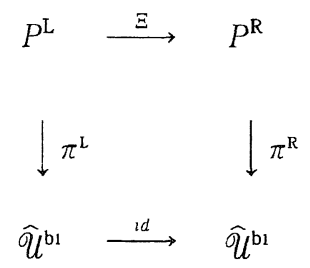

Let $\sigma^{\mathrm{L}}$ (respectively, $\sigma^{\mathrm{R}}$ ) denote the result of base-changing $\sigma$ via $i_{\mathrm{L}}$ (respectively, $i_{\mathrm{R}}$ ). Then by applying $\left(\sigma_{\mathrm{L}}\right)^{*}$, we can pull-back functions on $P^{\mathrm{L}}$ to biformal functions on $U^{\text {log }}$. Let $\mathscr{R}$ denote the étale sheaf of degree $\leq 1$ relative rational functions (as in [EGA IV], §20) on $P$ relative to $\pi: P \rightarrow U$ (i.e., the divisor of poles is flat of degree $\leq 1$ over $U$ ) that are regular in a neighborhood of the image of $\sigma$. Let $\alpha^{\mathrm{R}}: P^{\mathrm{R}} \rightarrow P$ denote the natural projection. Then it is easy to see that $\Xi^{-1}\left(\alpha^{R}\right)^{-1}(\mathscr{R})$ defines a sheaf of functions on $P^{\mathrm{L}}$ that are regular in a neighborhood of $\operatorname{Im}\left(\sigma^{\mathrm{L}}\right)$, so we can consider the subsheaf $\&$ of biformal functions on $L^{\text {rog }}$ which is the image of $\left(\sigma^{\mathrm{L}}\right)^{-1} \Xi^{-1}\left(\alpha^{\mathrm{R}}\right)^{-1}(\mathscr{R})$. One checks immediately that $\&$ defines a Schwarz structure, and, moreover, that since the 
connection $\nabla_{P}$ is necessarily integrable (since the dimension of $U$ over $S$ is one), $\mathscr{S}$ is automatically crystalline. Thus, in summary, we have the crystalline analogue of Theorem 2 of [Gunning]:

Theorem 1.7. If $f: U \rightarrow S$ is as above, then there is a natural one-to-one correspondence between crystalline Schwarz structures on $U^{\mathrm{log}}$ and isomorphism classes of $\mathbb{P}^{1}$-bundles with section and logarithmic connection $\left(\pi: P \rightarrow U ; \sigma: U \rightarrow P ; \nabla_{P}\right)$ on $U^{\mathrm{log}}$ whose associated Kodaira-Spencer morphism is an isomorphism. Moreover, under this correspondence, the crystalline Schwarz structures with nilpotent monodromy correspond precisely to the triples such that $\nabla_{P}$ has nilpotent monodromy' at the marked points.

Proof. We have already defined maps going in either direction. Thus, it suffices to see that these maps are inverse to each other. Now it is easy to see that if we start with a $\mathbb{P}^{1}$-bundle with section and logarithmic connection as above, construct the associated crystalline Schwarz structure $\&$. and then from that the associated $\mathbb{P}^{1}$-bundle $P_{\&}$ with section $\sigma_{\&}$ and logarithmic connection $\nabla_{P_{\&}}$, then we get back our original data. Thus. it suffices to show that the map that associates a $\mathbb{P}^{1}$-bundle with section and connection to a crystalline Schwarz structure is injective.

Let $\&$ and $\mathscr{S}^{\prime}$ be crystalline Schwarz structures on $U^{\text {log }}$. Suppose that we are given a horizontal isomorphism $\alpha$ between $P_{\&}$ and $P_{\&}$ that takes $\sigma_{\&}$ to $\sigma_{\&}$. Then $\alpha$ induces an isomorphism $\alpha_{B}$ of the $B$-torsors $B_{s}$ and $B \&$ : Let $\not A$ be the Ou-algebra $\mathcal{O}_{\hat{\tilde{u}}}$ "via the morphism $i_{\mathrm{R}}$. Since as sheaves with $B$-action, $B \& \cong L$

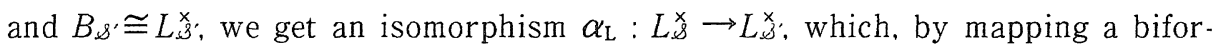
mal uniformizer $z \in \Gamma\left(U, L_{\xi}^{x}\right)$ (where $U \rightarrow X$ is étale) to the biformal uniformizer $\alpha_{\mathrm{L}}(z) \in \Gamma(U, L \Varangle) \subseteq \Gamma(U, \not{d})$, defines an automorphism $\alpha_{\mathscr{b}}$ of the

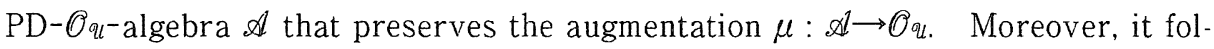
lows from the horizontality of $\alpha$ that $\alpha_{\&}$ is horizontal with respect to the standard logarithmic connection on $\mathscr{A}$. On the other hand, it is immediate that $\mathscr{A}$ does admit any nontrivial horizontal automorphisms (as a PD-O $u$-algebra) that preserve $\mu$. Thus, $\alpha_{\mathscr{A}}$ is the identity, and hence, $\mathscr{\&}$ and $\mathscr{S}^{\prime}$ must be the same subsheaf of $\mathscr{A}$. This completes the proof of the first statement. The last statement follows directly from the definitions.

\section{Schwarz Structures and Square Differentials}

We would like to use Theorem 1.7 to exhibit the space of Schwarz structures as a torsor over the square differentials. Let $\pi: P \rightarrow U$ be a $\mathbb{P}^{1}$-bundle. Then we shall denote by $\operatorname{Ad}(P)$ the vector bundle on $U$ (of rank three with trivial determinant) given by $\pi_{*} \tau_{P / U}$. When we consider marked points, it is not 
enough just to deal with $\mathbf{P}^{1}$-bundles; we must deal with $\mathbf{P}^{1}$-bundles equipped with parabolic structures, as in [Sesh]. Thus, if our divisor $D$ of marked points is given by sections $p_{1}, \ldots, p_{r}: S \rightarrow U$, we make the following

Definition 1.8. A $\mathbf{P}^{1}$-bundle with parabolic structure on $U^{\mathrm{log}}$ is defined to be a $\mathbb{P}^{1}$-bundle $\pi: P \rightarrow U$, together with sections $q_{\imath}: S \rightarrow P$ lying over $p_{i}$. A rank two vector bundle with parabolic structure on $U^{\text {log }}$ is a rank two vector bundle $\mathscr{E}$, together with a parabolic structure on $\mathbf{P}(\mathscr{E})$.

Let $\left(\pi: P \rightarrow U ; q_{1}, \ldots, q_{r}\right)$ be a $\mathbb{P}^{1}$-bundle with parabolic structure on $U^{\text {log }}$. Then we define the subsheaf $\operatorname{Ad}^{q}(P) \subseteq \operatorname{Ad}(P)$ to be the sheaf of sections that vanish at the $q_{i}$ 's. We define $\operatorname{Ad}^{\mathrm{c}}(P) \subseteq \operatorname{Ad}^{q}(P)$ to be the subsheaf of sections that vanish to second order (in the relative coordinate for $\pi$ ) at the $q_{i}$ 's. Suppose that we are given a section $\sigma: U \rightarrow P$ that avoids all the $q_{1}$. Let $\mathscr{L}=$ $\sigma^{*} \omega_{P / U}$. Then $\operatorname{Ad}(P)$ gets a filtration $0=F^{2}(\operatorname{Ad}(P)) \subseteq F^{1}(\operatorname{Ad}(P)) \subseteq F^{0}(\operatorname{Ad}(P))$ $\subseteq F^{-1}(\operatorname{Ad}(P))=\operatorname{Ad}(P)$ given by considering sections of $\tau_{P / U}$ that vanish to first or second order at $\sigma$. Thus, for $\operatorname{Ad}(P)$, we have:

$$
F^{1} \cong \mathscr{L} ; F^{0} / F^{1} \cong \mathscr{O}_{U} ; F^{-1} / F^{0} \cong \mathscr{L}^{-1}
$$

This filtration induces filtrations on $\operatorname{Ad}^{q}(P)$ and $\operatorname{Ad}^{c}(P)$. The subquotients are easily seen to be the following: For $\operatorname{Ad}^{q}(P)$, we have:

$$
F^{1} \cong \mathscr{L}(-D) ; F^{0} / F^{1} \cong \mathscr{O}_{U} ; F^{-1} / F^{0} \cong \mathscr{L}^{-1}
$$

For $\operatorname{Ad}^{c}(P)$, we have:

$$
F^{1} \cong \mathscr{L}(-D) ; F^{0} / F^{1} \cong \mathscr{O}_{U}(-D) ; F^{-1} / F^{0} \cong \mathscr{L}^{-1}
$$

Often, $\mathscr{L} \cong \omega_{U / s}^{\log }$. Thus, for computational purposes, it is convenient to note that $\omega_{U / S}^{\log }(-D)$ is none other than the relative dualizing sheaf of the morphism $f: U$ $\rightarrow S$.

Now let us assume that $\pi: P \rightarrow U$ is given by $\mathbb{P}\left(\mathscr{g} / \mathscr{g}^{[3]}\right)$, with the section $\sigma$ given by $\mathscr{g} / \mathscr{g}^{[3]} \rightarrow \mathscr{g} / \mathscr{g}^{[2]}$, and the $q_{\imath}$ given by the sections " $q_{s}$ " defined in the paragraph preceding Proposition 1.6. Let $\nabla_{P}$ be a logarithmic connection whose Kodaira-Spencer morphism at $\sigma$ is the identity and whose monodromy at the marked points is nilpotent and fixes the $q_{\imath}$. (It is not difficult to see that such $\nabla_{P}$ always exist étale locally on $U$.) Then any other such logarithmic connection $\nabla_{P}^{\prime}$ on $P \rightarrow U$ is given by adding to $\nabla_{P}$ a section of $F^{0}\left(\mathrm{Ad}^{\mathrm{c}}(P)\right) \otimes_{0_{v}} \omega_{U / \mathrm{S}}^{\log }$. On the other hand, the quadruples $\left(\pi ; \sigma ; q_{i} ; \nabla_{P}\right)$ and $\left(\pi ; \sigma ; q_{i} ; \nabla_{P}^{\prime}\right)$ are isomorphic if 
and only if $\nabla_{P}^{\prime}$ can be obtained from $\nabla_{P}$ by applying an automorphism $\alpha$ of $(\pi$; $\sigma ; q_{2}$ ) that preserves the conormal bundle to $\sigma$ (since both Kodaira-Spencer morphisms are the identity). Such an automorphism $\alpha$ is given by a section of $F^{\mathbf{1}}\left(\mathrm{Ad}^{q}\right.$ $(P))$. The effect of such an automorphism $\alpha$ on the connection $\nabla_{P}$ is given by adding to $\nabla_{P}$ the section of $\mathrm{Ad}^{c}(P) \otimes_{\mathscr{O}_{U}} \omega_{U / S}^{\log }$ obtained by applying the morphism $\operatorname{Ad}\left(\nabla_{P}\right): F^{1}\left(\operatorname{Ad}^{q}(P)\right) \rightarrow F^{0}\left(\operatorname{Ad}^{c}(P)\right) \otimes_{\mathscr{O}_{U}} \omega_{U / S}^{\mathrm{log}}$ (induced by the connection $\left.\nabla_{P}\right)$ to $\alpha$. Thus, we obtain that the set of isomorphism classes of quadruples $(\pi ; \sigma$; $\left.q_{i} ; \nabla_{P}\right)$ that, relative to the bijection of Theorem 1.7 , correspond to crystalline Schwarz structures with nilpotent monodromy are a torsor over the cokernel of Ad $\left(\nabla_{P}\right)$. On the other hand, by looking at the explicit representations of the subquotients of the filtrations on $\operatorname{Ad}^{q}(P)$ and $\operatorname{Ad}^{\mathrm{c}}(P)$ (given in the preceding paragraph), and using the fact that the Kodaira-Spencer morphism for $\nabla_{P}$ at $\sigma$ is an isomorphism. we obtain that

$$
\operatorname{Coker}\left(\operatorname{Ad}\left(\nabla_{P}\right)\right) \cong\left(\omega_{U / S}^{\log }\right) \otimes 2(-D)
$$

(We remark that here one uses the fact that $p$ is odd, for when one computes Ad $\left(\nabla_{P}\right)$ from $\nabla_{P}$, certain factors of 2 appear, and in order to get the above isomorphism, one needs for those factors of 2 to be invertible.)

In other words, we have proven the following result:

Therrem 1.9. The étale sheaf of crystalline Schwarz structures on $U^{\log }$ with nilpotent monodromy is naturally a torsor over the sheaf $\left(\omega_{U / S}^{\log }\right) \otimes 2(-D)$.

\section{Normalized $\mathbb{P}^{1}$-burndles with Commection}

Let us consider the $\mathbb{P}^{1}$-bundle $\pi: P=\mathbb{P}\left(\mathscr{g} / \mathscr{g}^{[3]}\right) \rightarrow U$, and section $\sigma: U \rightarrow P$ given by $\mathscr{J} / \mathscr{g}^{[3]} \rightarrow \mathscr{g} / \mathscr{g}^{[2]}$, without any connection. Now, just as in the proof of Proposition 1.4 , by taking residues, we obtain a natural duality between $\mathscr{O}_{P}(-\sigma)$ $/ \mathscr{O}_{P}(-3 \sigma)$ and $\pi_{*} \omega_{P / U}(3 \sigma)$ that respects the natural filtration on the two bundles. Let $Q=\mathbb{P}\left(\left(\mathscr{g} / \mathscr{g}^{[3]}\right) \vee\right)$. As $U$-schemes, we may identify $Q$ and $P$. Let $\mathscr{O}_{Q}(1)$ denote the line bundle obtained from the definition of the projectivization; thus $\pi_{*} \mathscr{O}_{Q}(1)=\left(\mathscr{J} / \mathscr{g}^{[3]}\right) \vee$. Let $\mathscr{L}=\pi_{*}\left\{\mathscr{O}_{Q}(1) \otimes_{\mathcal{O}_{Q}} \tau_{P / U}(-3 \sigma)\right\}$. Then $\mathscr{L}$ is a line bundle on $U$ and $\left(\mathscr{I} / \mathscr{g}^{[3]}\right) \vee=\mathscr{L} \otimes_{\hat{O}_{u}} \pi_{*} \omega_{P / U}(3 \sigma)$. Thus, we obtain a natural isomorphism $\mathscr{O}_{P}(-\sigma) / \mathscr{O}_{P}(-3 \sigma) \cong\left(\mathscr{g} / \mathscr{g}^{[3]}\right) \otimes_{\mathscr{O}_{U}} \mathscr{L}$ that respects filtrations. If we then look at the quotients of both sides by their respective rank one subbundles (that make up the filtrations), we obtain an isomorphism between $\omega_{U / S}^{\log }$ $\cong \mathscr{O}_{P}(-\sigma) / \mathscr{O}_{P}(-2 \sigma) \cong\left(\mathscr{g} / \mathscr{g}^{[2]}\right) \otimes \mathscr{L} \cong \omega_{U / S}^{\log } \otimes \mathscr{L}$. That is, we get a natural trivialization $\mathfrak{O}_{U} \cong \mathscr{L}$ of $\mathscr{L}$. In summary, we see that without any connection, we 
have constructed a natural filtration-preserving isomorphism:

$$
\gamma: \mathscr{O}_{P}(-\sigma) / \mathscr{O}_{P}(-3 \sigma) \cong \mathscr{g} / \mathscr{g}^{[3]}
$$

Now let us suppose that we have a logarithmic connection $\nabla_{P}$ on $\pi$ whose Kodaira-Spencer morphism at $\sigma$ is an isomorphism. Then we get a commutative diagram like the one preceding Theorem 1.7. Pulling back by $\alpha^{\mathrm{R}}$, then $\Xi$, and finally by $\sigma^{\mathrm{L}}$, we thus see that $\nabla_{P}$ induces an isomorphism:

$$
\zeta\left(\nabla_{P}\right): \mathscr{O}_{P}(-\sigma) / \mathscr{O}_{P}(-3 \sigma) \cong \mathscr{g} / \mathscr{g}^{[3]}
$$

Now we saw above (Theorem 1.7) that $\nabla_{P}$ defines a Schwarz structure. But one "loose end" relative to the statement of Theorem 1.7 is that although Schwarz structures have no automorphisms, triples consisting of projective bundles with a section and a connection can have automorphisms. These automorphisms were the cause of the phenomenon (observed just before the statement of Theorem 1.9) that many different $\nabla_{P}$ can give rise to the same Schwarz structure. Thus, it is convenient to have some sort of notion of a "normalized $\nabla_{P}$ " such that each Schwarz structure arises from a unique normalized $\nabla_{P}$. We choose the normalization as follows:

Definition 1.10. We say that $\nabla_{P}$ is normalized if $\gamma$ and $\zeta\left(\nabla_{P}\right)$ are inverse to each other.

Now as a formal consequence of this definition, we observe that we obtain the following normalized version of Theorem 1.7:

Theorem 1.11. If $f: U \rightarrow S$ is as above, then there is a natural one-to-one correspondence between crystalline Schwarz structures on $U^{\mathrm{log}}$ and normalized logar. ithmic connections on the $\mathbb{P}^{1}$-bundle $\pi: \mathbb{P}\left(\mathscr{g} / \mathscr{g}^{[3]}\right) \rightarrow U$ whose associated Kodaira-Spencer morphism at the section $\sigma: U \rightarrow P$ (defined by $\mathscr{g} / \mathscr{g}^{[3]} \rightarrow \mathscr{g} / \mathscr{g}^{[2]}$ ) is an isomorphism. Moreover. under this correspondence, the crystalline Schwarz struc. tures with nilpotent monodromy correspond precisely to the triples such that $\nabla_{P}$ has nilpotent monodromy at the marked points.

\section{The Schwarzian Derivative}

Before proceeding, it is interesting to note that, as the name suggests, a crystalline Schwarz structure $\&$ allows one to define a Schwarzian derivative $d_{s}$, as follows. Let $\omega_{U^{\mathrm{108}} / S^{108}}^{{ }_{10}} \subseteq \omega_{U^{108} / S^{108}}$ denote the subsheaf consisting of sections that locally generate $\omega_{U^{100} / S^{108}}$ as an $\mathscr{O}_{U}$-module. Let $\mathscr{O}_{U}^{\#} \subseteq \mathscr{O}_{U}$ be the subsheaf consist- 


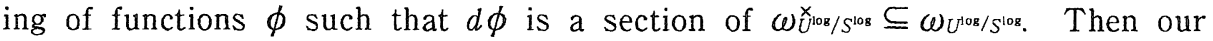
Schwarzian derivative will be a morphism of sheaves of sets:

$$
d \&: \mathscr{O}_{U}^{\#} \rightarrow \omega_{U}^{\otimes 2} \mathbb{Z}_{/} / S^{\log }
$$

Let $\theta$ be a section of $\mathscr{O}_{U}^{*}$ over some étale $V \rightarrow U$. Let us denote by $j_{\theta} \in \mathscr{g} / \mathscr{g}^{[3]}(V)$ the 2-jet of $\theta$ (i.e., the Taylor expansion out to second order, modulo the constant term). By the definition of $\mathscr{O}_{U}^{\#}$, the image of $j_{\theta}$ in $\mathscr{g} / \mathscr{g}^{[2]}(V)$ is a local generator of the sheaf $\mathscr{g} / \mathscr{g}^{[2]}$. By Proposition $1.6, j_{\theta}$ then defines a section $s_{\theta}: V \rightarrow P_{\&}$. Taking the Kodaira-Spencer map of this section then defines an $\mathscr{O}_{V}$-linear morphism from $\tau_{U^{\mathrm{jog}} / S^{\mathrm{log}}}$ to the conormal bundle to $s_{\theta}$, which is simply $\omega_{U^{108} / S^{\text {log }}}$. This $\mathscr{O}_{V}$-linear morphism is thus given by multiplication by a section of $\omega_{U^{\text {log }} / S^{\text {log }}}^{\otimes 2}$ which we take to be $d_{\&}(\theta)$. A simple calculation reveals that

Proposition 1.12. If (as in Theorem 1.9) one modifies the Schwarz structure $\&$ by adding the square differential $\delta \in\left[\left(\omega_{U / S}^{\log }\right)^{\otimes 2}(-D)\right](U)$ to obtain a Schwarz structure $\mathscr{\&}^{\prime}$, then $d_{\mathscr{\&}}(\theta)=d_{\mathscr{\vartheta}^{\prime}}(\theta)+\delta$.

We also have a biformal version of the Schwarzian derivative. Namely, we let $\mathscr{O}_{\tilde{W}^{\mathrm{b}}}^{\#}$ be the subsheaf of $\mathscr{O}_{\hat{q} b^{b}}$ consisting of biformal functions $\phi$ that are of the form $u+c$, where $u$ is a biformal uniformizer, and $c$ is a biformal constant. Then we get a morphism of sheaves of sets:

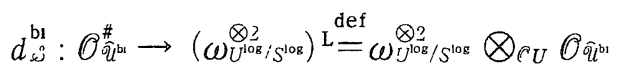

defined as follows: If $\theta$ is a section of $\mathscr{O}_{\mathfrak{U}^{\mathrm{m}}}^{\#}$ over some étale $V \rightarrow U$, we let $j_{\theta}$ be the section of $\mathscr{g} / \mathscr{g}^{[3]} \otimes_{\hat{\kappa}_{U}} \mathcal{O}_{\hat{\mathcal{U}}^{b}}$ which is the 2-jet of $\theta$. Thus, $j_{\theta}$ defines a section of $s_{\theta}$ of $P_{\&}^{L}$ whose Kodaira-Spencer map is given by multiplication by a section of $\left(\omega_{U^{108} / S^{108}}^{\otimes 2}\right)^{\mathrm{L}}$, which we take as $d_{\star s}^{\mathrm{bl}}(\theta)$. Note that $d_{\lrcorner}^{\mathrm{bi}}(\theta)=i_{\mathrm{L}}\left(d_{. s}(\theta)\right)$, and that if we modify the Schwarz structure by adding a square differential $\delta$, then

$$
d_{\& \delta}^{\mathrm{bl}}(\theta)=d_{\& 8^{\prime}}^{\mathrm{bl}}(\theta)+i_{\mathrm{L}}(\delta)
$$

Remark. In the Appendix to this Chapter, we show that the definition just given for the Schwarzian derivative coincides with one-half the classical Schwarzian derivative, when $U$ is the projective line.

For the biformal version of the Schwarzian, we have an analogue of the classical result that the Schwarzian vanishes exactly on the formal functions 
that make up the projective structure of a Riemann surface. Indeed, let $\Xi: P_{\&}^{\mathrm{L}}$ $\rightarrow P_{\&}^{\mathrm{R}}$ be the isomorphism defined by the connection $\nabla_{P_{\&}}$; let $\alpha^{\mathrm{R}}: P_{\&}^{\mathrm{R}} \rightarrow P_{\&}$ be the natural projection; and let $\zeta: \widehat{U}^{\mathrm{bl}} \stackrel{\text { def }}{=} \operatorname{Spec}\left(\widehat{O}_{\widehat{U}^{\mathrm{b}}}\right) \rightarrow P_{\&}$ be the morphism obtained by composing $\sigma_{\&}^{\mathrm{L}}: \hat{\mathcal{U}}^{\mathrm{bl}} \rightarrow P_{s}^{\mathrm{L}}$ with $\Xi$ and then $\alpha^{\mathrm{R}}$. Since the definition of $d_{\&}$ is functorial, $d_{\Varangle}$ applied to a function pulled back by $\zeta$ is $\zeta^{-1}$ of the " $d_{\S}$ " computed for projective bundles in the Appendix, i.e., one-half the classical Schwarzian. Thus, if $\theta$ is a section of $\mathscr{S}$, then $\theta$ is the pull-back by $\zeta$ of a (degree $\leq 1$ ) relative rational function for $P_{s} \rightarrow U$, so $d_{s}^{\text {b1 }}(\theta)=0$.

Conversely, suppose that $d_{\lrcorner}^{\mathrm{bl}}(\theta)=0$. Then the statement that $d_{\star s}^{\text {bl }}(\theta)=0$ means that $s_{\theta}$ is a horizontal section of $P_{s s}^{\mathrm{L}}$. Thus it follows from the definition of a connection, together with the Poincare Lemma in crystalline cohomology (see. e.g., [Kato], $\S 6$ for the $\log$ version) that $\Xi\left(s_{\theta}\right)$ is the pull-back via $\alpha^{\mathrm{R}}$ of a section $t_{\theta}$ of $P_{s}$. Now (after possible étale localization), we can find a (degree $\leq 1$ ) relative rational function $\phi$ for $P_{\&} \rightarrow U$ whose 2-jet at $\sigma_{\&}$ is given by the section $t_{\theta}$. Since $\zeta$ maps the diagonal in $\widehat{\mathscr{U}}^{\mathrm{b} 1}$ to $\sigma_{\&}^{\mathrm{R}}$, and the formation of 2 -jets is functorial, it thus follows that the 2-jet of $\phi \stackrel{\text { def }}{=} \zeta^{-1}(\phi)$ defines a section $s_{\psi}$ of $P_{s}^{L}$ which is equal to $s_{\theta}$ when restricted to the diagonal $\mathscr{U} \subseteq \widehat{\mathscr{U}}^{\text {bi }}$. But since both $s_{\phi}$ and $s_{\theta}$ are horizontal, they must be equal. The biformal functions $\phi$ and $\theta$ thus have 2 -jets that define the same "line" in $g / g^{[3]}$. Let $z$ be a local coordinate on $U$. Let us denote by successive primes the derivatives of biformal functions (i.e., taken on the left) with respect to $z$. Then we obtain that $\phi^{\prime}$ and $\theta^{\prime}$ are both invertible biformal functions such that $\phi^{\prime \prime} \cdot \theta^{\prime}=\phi^{\prime} \cdot \theta^{\prime}$. It thus follows that $\left(\phi^{\prime} / \theta^{\prime}\right)^{\prime}=0$, so $\theta^{\prime}=a \phi^{\prime}$, where $a$ is an invertible biformal constant. Thus, $\theta=a \phi+b$, where $b$ is a biformal constant. Since, by construction, $\phi \in \varnothing(V)$. it follows that $\theta \in \&(V)$. Thus, we obtain the following "crystalline Schwarzian Poincaré Lemma:"

Theorem 1.13. If $\theta$ is a biformal function, then $d_{\&}^{\mathrm{b} 1}(\theta)=0$ if and only if $\theta$ is a section of $\&$.

\section{§2. Indigenous Bundles}

In this Section, we globalize the local considerations of $\S 1$, and are thus led to introduce "indigenous bundles" (as in [Gunning]). Let $S^{\log }$ be a fine log scheme, whose underlying scheme is connected noetherian. Let $f^{\log }: X^{\log } \rightarrow S^{\log }$ be proper, geometrically connected, and locally stable of dimension one. (Note that the first two conditions are actually conditions on the underlying scheme morphism $f$.) We assume that the fibers of $f: X \rightarrow S$ have arithmetic genus $g \geq 0$, 
and exactly $r \geq 0$ marked points (as in Definition 1.1 - note that these may only be defined étale locally, however).

\section{Basic Definitions and Examples}

If $2 g-2+r \geq 1$, then let $\bar{M}_{g, r}$ be the moduli stack of stable curves of genus $g$. with $r$ marked points, over $\mathbb{Z}$, and let $\zeta: \mathscr{C} \rightarrow \bar{M}_{g, r}$ be the universal curve, with its $r$ marked points $s_{1}, \ldots, s_{r}: \bar{M}_{g, r} \rightarrow \mathscr{C}$. Note that $\bar{M}_{g, r}$ has a natural log structure given by the divisor at infinity. Denote the resulting log stack $\bar{M}_{g, r}^{\text {log }}$. Also, by taking the divisor which is union of the $s_{t}$ and the pull-back of the divisor at infinity of $\bar{M}_{g, r}$, we get a $\log$ structure on $\mathscr{C}$; we call the resulting log stack $\mathscr{C}^{\text {log }}$. Also, $\zeta: \mathscr{C} \rightarrow \bar{M}_{g, r}$, extends naturally to a morphism of log stacks $\zeta^{\log }: \mathscr{C}^{\log } \longrightarrow \bar{M}_{g, r}^{\mathrm{log}}$

Definition 2.1. We shall say that $f^{\text {log }}: X^{\text {log }} \rightarrow S^{\text {log }}$ is stable if there exists a classifying morphism $\phi^{\log }: S^{\log } \rightarrow \overline{\mathcal{M}}_{g, r}^{\log }$ such that $X^{\log } \cong S^{\log } \times{\overline{M_{M g}^{10 g}}}_{\mathscr{C}^{10 g}}$.

Ultimately, we shall be concerned mainly with the case where $f^{\text {log }}$ is stable, but it is useful to realize that the definition, as well as many of the first properties, of indigenous bundles can be made without these assumptions.

Let $\pi: P \rightarrow X$ be a $\mathbb{P}^{1}$-bundle. If $\sigma: X \rightarrow P$ is a section, then we call the canonical height of $\sigma$ the number $\frac{1}{2} \operatorname{deg}_{X / S}\left(\sigma^{*} \tau_{P / X}\right)$, where $\operatorname{deg}_{X / S}$ denotes the relative degree over $S$ of a line bundle on $X$, and $\tau_{P / X}$ is the relative tangent bundle of $\pi$. If $\nabla_{P}$ is a logarithmic connection on $P$, then we call the morphism $\tau_{\gamma^{100} / S^{\text {log }}}$ $\rightarrow \sigma^{*} \tau_{P / X}$ given by differentiating $\sigma$ by means of $\nabla_{P}$ the Kodaira-Spencer morphism at $\sigma$ relative to $\nabla_{P}$. Often, instead of dealing with $\mathbb{P}^{1}$-bundles with logarithmic connections, it will be more convenient to deal vector bundles: Thus, let $\mathscr{E}$ be a vector bundle equipped with a logarithmic connection $\nabla_{\mathscr{g}}$, whose rank is two and whose determinant is trivial. Then Theorem 1.7 motivates the following

Definimition 2.2. We shall say that $\left(\pi: P \rightarrow X, \nabla_{P}\right)$ is an indigenous bundle on $X^{\log }$ if the monodromy at the marked points (which exist étale locally) is nilpotent, and there exists a section $\sigma: X \rightarrow P$ of $\pi$ such that the KodairaSpencer morphism at $\sigma$ with respect to $\nabla_{P}$ is an isomorphism. We shall say that $\left(\mathscr{E}, \nabla_{\mathscr{E}}\right)$ is an indigenous vector bundle on $X^{\mathrm{log}}$ if the associated $\mathbb{P}^{1}$-bundle with logarithmic connection $(\mathbb{P}(\mathscr{E}) \rightarrow X, \nabla \mathbf{P}(\mathscr{E}))$ is an indigenous $\mathbb{P}^{1}$-bundle. We shall say that $P \rightarrow X$ (respectively, $\mathscr{E}$ ) is intrinsic if there exists a logarithmic connection $\nabla_{P}$ (respectively, $\left.\nabla_{\mathscr{g}}\right)$ on $P \rightarrow X$ (respectively, $\left.\mathscr{E}\right)$ that makes $(P \rightarrow X$. 
$\left.\nabla_{P}\right)$ (respectively, $\left(\mathscr{E}, \nabla_{\mathscr{E}}\right)$ ) indigenous. We shall say that $P \rightarrow X$ (respectively, $\mathscr{E})$ is locally intrinsic if it is intrinsic étale locally on $S$.

Thus, in the vector bundle case, $\left(\mathscr{E}, \nabla_{\mathscr{E}}\right)$ is indigenous if $\nabla_{\mathscr{E}}$ has nilpotent monodromy at the marked points, and there exists a rank one subbundle $F^{0}(\mathscr{E})$ $\subseteq \mathscr{E}$ such that the Kodaira-Spencer morphism $F^{0}(\mathscr{E}) \rightarrow \omega_{X^{108} / S^{108}} \bigotimes_{\mathscr{O} \times}\left(\mathscr{E} / F^{0}(\mathscr{E})\right)$ (induced by $\nabla_{\mathscr{E}}$ ) is an isomorphism.

So far we have been discussing the hyperbolic case $(2 g-2+r \geq 1)$; however, one can make the same definition for curves that are not hyperbolic.

Example 1. Suppose that $f: X \rightarrow S$ is smooth, with no marked points, and that $g=0$. Thus. $f$ is a $\mathbb{P}^{1}$-bundle. Then the $\mathbb{P}^{1}$-bundle given by $X \times{ }_{s} X \rightarrow X$ has a natural trivial connection, together with a natural section, the diagonal section. It is trivial to see that this triple satisfies the required properties for an indigenous bundle.

Example 2. Suppose that $f^{\text {log }}: X^{\log } \rightarrow S^{\text {log }}$ has no marked points, and that its fibers all have arithmetic genus one. Then consider the bundle $\mathscr{E}=\omega_{X / S} \oplus$ $\mathscr{O}_{X}$ (where $\omega_{X / S}$ is the relative dualizing sheaf). Let $\mathscr{L}=f_{*} \omega_{X / S}$. Thus, $\mathscr{L}$ is a line bundle on $S$, and $f^{*} \mathscr{L} \cong \omega_{X / S}$. In particular, there exists on $\omega_{X / S}$ a "trivial connection" $\nabla_{\omega}$ obtained from tensoring the trivial connection on $\mathscr{O}_{X}$ with $f^{*} \mathscr{L}$. Let $\nabla_{\mathscr{E}}^{\prime}$ be the connection on $\mathscr{E}$ which is the direct sum of $\nabla_{\omega}$ and the trivial connection on $\mathscr{O}_{X}$. Let $\nabla_{\mathscr{E}}$ be the connection on $\mathscr{E}$ given by adding to $\nabla_{\mathscr{g}}^{\prime}$ the section of $\operatorname{End}(\mathscr{E}) \otimes \omega_{X / S}$ given by projecting $\mathscr{E} \rightarrow \omega_{X / S} \cong\left(0, \mathscr{O}_{X}\right) \otimes \omega_{X / S} \subseteq \mathscr{E} \otimes \omega_{X / S}$. Then one checks easily that if we take $\left(P, \nabla_{P}\right)=\mathbb{P}\left(\mathscr{E}, \nabla_{\mathscr{E}}\right)$, and $\sigma: X \rightarrow P$ to be given bv $\left(\omega_{X / s}, 0\right) \subseteq \mathscr{E}$, then we obtain an indigenous bundle on $X^{\log }$.

Example 3. Let $S^{\log }=\operatorname{Spec}(\mathbb{Z})$ (with the trivial log structure); $X^{\log }=\bar{M}_{1,1}$ (the moduli stack of one-pointed curves of genus one over $\mathbb{Z}$ ) with its natural $\log$ structure. Let $\mathscr{E}$ be the vector bundle of rank two on $X$ which is the first de Rham cohomology module of the universal one-pointed curve of genus one. Then $\mathscr{E}$ has a natural logarithmic connection $\nabla \mathscr{E}$, the so-called "Gauss-Manin connection." There is also a natural Hodge filtration $F^{1}(\mathscr{E}) \subseteq \mathscr{E}$, which defines a section $\sigma: X \rightarrow P=\stackrel{\text { def }}{=} \mathbf{P}(\mathscr{E})$. The pair $\left(\mathscr{E}, \nabla_{\mathscr{E}}\right)$ forms the prototypical example of an indigenous bundle on $X^{\mathrm{log}}$.

\section{First Properties}

We now proceed to examine basic properties of such bundles. 
Proposition 2.3. If $f^{\mathrm{log}}: X^{\mathrm{log}} \rightarrow S^{\mathrm{log}}$ and $h^{\mathrm{log}}: Y^{\mathrm{log}} \rightarrow S^{\mathrm{log}}$ are as stipulated at the beginning of the Section, and $\zeta^{\mathrm{log}}: Y^{\mathrm{log}} \rightarrow X^{\mathrm{log}}$ is a log étale morphism of log schemes over $S^{\log }$ that sends marked points to marked points, then the pull-back via $\zeta^{\mathrm{log}}$ of any indigenous bundle is again indigenous.

Proof. This follows from the definitions.

Proposition 2.4. If $\pi: P \rightarrow X$ is intrinsic, then the section $\sigma: X \rightarrow P$ is of canonical height $1-g-\frac{1}{2} r$. If $X^{\log } \rightarrow S^{\log }$ is a stable curve (so, in particular, $2 g-$ $2+r \geq 1$ ), then $\sigma$ is the unique section of $\pi$ of canonical height $1-g-\frac{1}{2} r$. We shall refer to $\sigma$ as the Hodge section of $\pi: P \rightarrow X$.

Proof. The fact that the canonical height of $\sigma$ is $1-g-\frac{1}{2} r$ follows from the fact that the Kodaira-Spencer morphism is an isomorphism. Now suppose that $X^{\log } \rightarrow S^{\log }$ is stable. Let us first assume that $S$ is the spectrum of an algebraically closed field. Suppose that $\sigma^{\prime}: X \rightarrow P$ also has canonical height $1-g-\frac{1}{2} r$. Then it follows that its restriction to some irreducible component of $X$ has nega tive canonical height. Since the restriction of $\sigma^{*} \tau_{P / X}$ to any irreducible component has negative degree, it follows immediately from considering intersection numbers on $P$, together with the definition of "canonical height," that there can not exist two distinct sections of negative canonical height over that irreducible component. Thus, $\sigma$ and $\sigma^{\prime}$ must agree over that irreducible component. Now if there are any other irreducible components in $X$, then $\sigma^{\prime}$ must have negative canonical height over some other irreducible component of $X$, in order for its canonical height over all of $X$ to be $1-g-\frac{1}{2} r$. Thus, repeating this argument shows that $\sigma=\sigma^{\prime}$. Finally, let us observe that the space of deformations of $\sigma$ is given by $H^{0}\left(X, \sigma^{*} \tau_{P / X}\right)$, which is zero, since $\sigma^{*} \tau_{P / X}$ has negative degree on every irreducible component of $X$. The result for general $S$ then follows immediately from this by deformation theory.

Now let us assume for the rest of the Section (unless stated otherwise) that there exists an odd prime $p$ which is nilpotent on $S$, together with a PD-ideal $\mathscr{I}$ $\subseteq \mathfrak{O}_{s}$.

Proposition 2.5. If $\pi: P \rightarrow X$ is intrinsic, then $P \cong \mathbb{P}\left(\mathscr{E} / \mathscr{g}^{[3]}\right)$ (where $\mathscr{J}$ de fines the diagonal in $\left.\mathscr{K}^{\mathrm{bl}}\right)$. Moreover, for any connection on $\pi$ that makes it indige. nous, the monodromy al a marked point $s: S \rightarrow X$ fixes the section $q_{s}: S \rightarrow \mathbb{P}$ 
$\left(\mathscr{g} / \mathscr{g}^{[3]}\right)$ of Proposition 1.6.

Proof. This follows from Theorem 1.7 and Propositions 1.3 and 1.6. Note that the second statement uses the fact that $p$ is odd.

Proposition 2.6. Suppose that the number of marked points plus nodes on any geometric irreducible component of a fiber of $X \rightarrow S$ is even. Let $(\pi: P \rightarrow X$, $\left.\nabla_{P}\right)$ be indigenous on $X^{\mathrm{log}}$. Then étale locally on $S$, there exists an indigenous vector bundle whose projectivization is $\left(\pi: P \rightarrow X, \nabla_{P}\right)$. Moreover, such an $\left(\mathscr{E}, \nabla_{\mathscr{E}}\right)$ is unique up to tensor product with a line bundle with connection $\left(\mathscr{L}, \nabla_{\mathscr{L}}\right)$ on $X$ whose square is trivial.

Proof. Consider the relative anticanonical bundle $\tau_{P / X}$ on $P$. By Proposition 2.5, (after étale localization on $S$ ) there exists a line bundle $\mathscr{G}$ on $P$ whose square is $\tau_{P / X}$. Now let us note that since the construction of the anticanonical bundle is canonical, it follows that the connection $\nabla_{P}$ on the $\mathbb{P}^{1}$-bundle induces a connection on the polarized $\mathbb{P}^{1}$-bundle $\left(\pi: P \rightarrow X, \tau_{P / X}\right)$. Moreover, since the "moduli space" of line bundles $\mathscr{G}$ whose square is $\tau_{P / X}$ is étale over $X$, it follows that the connection $\nabla_{P}$ on the $\mathbb{P}^{1}$-bundle $\pi: P \rightarrow X$ in fact induces a connection on the polarized $\mathbb{P}^{1}$-bundle $(\pi: P \rightarrow X, \mathscr{G})$. Thus, we get a connection $\nabla_{\mathscr{E}}$ on $\mathscr{E}=\pi_{*}^{\text {def }} \mathscr{G}$. Moreover, on $P$, we have a natural exact sequence $0 \rightarrow \omega_{P, X} \rightarrow\left(\pi^{* \mathscr{E}}\right)$ $\otimes \mathscr{G}^{-1} \rightarrow \mathscr{O}_{P} \rightarrow 0$, which induces an isomorphism det $\left(\pi^{* \mathscr{E}}\right) \cong \mathscr{O}_{P}$, hence an isomorphism $\operatorname{det}(\mathscr{\mathscr { E }}) \cong \mathscr{O}_{X}$, which is easily seen to be horizontal. Finally, it is clear that the projectivization of $\left(\mathscr{E}, \nabla_{\mathscr{E}}\right)$ is isomorphic to $\left(\pi: P \rightarrow X, \nabla_{P}\right)$.

Now suppose that both $\left(\mathscr{E}, \nabla_{g}\right)$ and $\left(\mathscr{E}^{\prime}, \nabla g^{\prime}\right)$ have the same projectivization $\left(\pi: P \rightarrow X, \nabla_{P}\right)$. Then $\mathscr{E}$ defines a line bundle $\mathscr{G}$ on $P$ whose square is $\tau_{P / X}$ and such that $\nabla_{P}$ induces a connection on the polarized $\mathbb{P}^{1}$-bundle $(\pi: P \rightarrow X, \mathscr{G})$. Similarly, $\mathscr{E}^{\prime}$ defines a line bundle $\mathscr{G}^{\prime}$ on $P$. Since we have horizontal isomorphisms $\mathscr{G}^{\otimes 2} \cong \tau_{P / X}$ and $\left(\mathscr{G}^{\prime}\right)^{\otimes 2} \cong \tau_{P / X}$, it follows that if we let $\mathscr{L}=\pi_{*}\left(\left(\mathscr{G}^{\prime}\right)^{-1} \otimes \mathscr{G}\right)$. then $\mathscr{L}$ gets a natural connection $\nabla \mathscr{L}$ such that the square of $\left(\mathscr{L}, \nabla_{\mathscr{L}}\right)$ is trivial. Moreover, $\left(\mathscr{E}, \nabla_{\mathscr{E}}\right) \cong\left(\mathscr{E}^{\prime}, \nabla_{\mathscr{E}^{\prime}}\right) \otimes\left(\mathscr{L}, \nabla_{\mathscr{L}}\right)$. This completes the proof.

In summary, the above Proposition tells us that (under the evenness assumption) up to étale localization on the base, it is essentially the same thing to give an indigenous $\mathbb{P}^{1}$-bundle or an indigenous vector bundle. Thus, in the future, we shall frequently simply speak of "indigenous bundles." The same goes for intrinsic bundles.

\section{Existence and de Rham Cohomology}

The next step is to prove the existence of indigenous bundles, and to parametrize them. We begin with the proof of existence. For the rest of this Sec- 
tion, we shall assume that $f^{\text {log }}$ is stable. Thus, in particular. $2 g-2+r \geq 1$.

Theorem 2.7. For any r-pointed stable curve $X^{\log } \rightarrow S^{\log }$ of genus $g$, the $\mathbb{P}^{1}$-bundle $\mathbb{P}\left(\mathscr{g} / \mathscr{g}^{[3]}\right)$ is locally intrinsic.

Proof. From Theorem 1.9, we know that the obstruction to the existence of a crystalline Schwarz structure with nilpotent monodromy on $X^{\text {log }}$ (locally on $S)$ is given by a section of $\mathbb{R}^{1} f_{*}\left(\omega_{X / S}^{\log }\right) \otimes 2(-D)$ over $S$. On the other hand, by Serre duality, $\mathbb{R}^{1} f_{*}\left(\omega_{X / S}^{\log }\right) \otimes 2(-D)$ is isomorphic to the dual of $f_{*} \tau_{X^{\log } / S^{\log }}=0$, since the curve is stable. The Theorem now follows from Proposition 1.6 and Theorem 1.7.

Next we wish to compute the de Rham cohomology of the $\mathbb{P}^{1}$-bundle with parabolic structure $\left(\pi ; q_{\imath}\right)$. Note that the exterior differential operator maps $\operatorname{Ad}(P)$ (respectively, $\operatorname{Ad}^{q}(P)$ ) into $\operatorname{Ad}^{q}(P)$ (respectively, $\left.\operatorname{Ad}^{c}(P)\right)$. We define the parabolic de Rham cohomology (respectively, with compact supports) of $\operatorname{Ad}(P)$ to be the hypercohomology of the complex $\operatorname{Ad}(P) \rightarrow \mathrm{Ad}^{q}(P) \otimes \omega_{X / S}^{\log }$ (respectively, $\left.\operatorname{Ad}^{q}(P) \rightarrow \operatorname{Ad}^{c}(P) \otimes \omega_{X / S}^{\text {log }}\right)$.

Theorem 2.8. Let $\left(P, \nabla_{P}\right)$ be an indigenous bundle on an r-pointed stable curve $f^{\mathrm{log}}: X^{\mathrm{log}} \rightarrow S^{\mathrm{log}}$ of genus $g$. Then the de Rham cohomology of $\operatorname{Ad}(P)$ with its natural connection (induced by $\nabla_{P}$ ) is as follows:

(1) For cohomology without compact supports, we have $\left(f_{\mathrm{DR}}\right) *(\mathrm{Ad}(P))=$ $\mathbb{R}^{2}\left(f_{\mathrm{DR}}\right) *(\mathrm{Ad}(P))=0$; and we have a natural exact sequence

$$
0 \rightarrow f_{*}\left(\omega_{X / S}^{\log }\right)^{\otimes 2}(-D) \rightarrow \mathbb{R}^{1}\left(f_{\mathrm{DR}}\right) *(\operatorname{Ad}(P)) \rightarrow \mathbb{R}^{1} f_{*} \tau_{\lambda}{ }^{\log / S^{\log }} \rightarrow 0
$$

(2) For cohomology with compact supports, we have (for all $i \geq 0$ ) a natu ral isomorphism

$$
\mathbb{R}^{i}\left(f_{\mathrm{DR}}\right)_{\mathrm{c}, *}(\operatorname{Ad}(P)) \cong \mathbb{R}^{i}\left(f_{\mathrm{DR}}\right)_{*}(\operatorname{Ad}(P))
$$

In particular, $\left(P ; \nabla_{P}\right)$ has no nontrivial automorphisms.

Proof. To compute the de Rham cohomology, one uses the long exact cohomology sequences induced by the filtrations considered above, plus the fact that the Kodaira-Spencer morphism is an isomorphism. Now let $\alpha$ be an automorphism. Since $\left(f_{\mathrm{DR}}\right) *(\mathrm{Ad}(P))=0$, all infinitesimal automorphisms must vanish, so we may work over an algebraically closed field. By passing to a tamely ramified covering of $X$ ramified only at the marked points and nodes, we may 
assume that the hypotheses of Proposition 2.6 are satisfied. Then let $\mathscr{L}$ be a line bundle on $P$ whose square is $\tau_{P / X}$. Since $\mathscr{L} \cong \mathscr{O}_{P}(\sigma) \bigotimes_{\mathscr{O}_{S}} \mathcal{M}$ (for a line bundle $\mathcal{M}$ on $S$ ), and $\alpha$ always preserves $\sigma$, it follows that $\alpha$ preserves $\mathscr{L}$. Thus, $\alpha$ arises from a horizontal section of End $\left(\pi_{*} \mathscr{L}\right)=\mathscr{O}_{X} \oplus \operatorname{Ad}(P)$, hence is induced by multiplying $\pi_{*} \mathscr{L}$ by a section of $\mathscr{O}_{s}$. Thus, $\alpha$ is the identity, as desired.

Finally, combining what we have done in this Section with Theorem 1.7, we obtain:

Corollary 2.9. Let $f^{\log }: X^{\log } \rightarrow S^{\log }$ be an r-pointed stable curve of genus $g$. Then the set of crystalline Schwarz structures on $X$ with nilpotent monodromy is in one-to-one correspondence the set of isomorphism classes of indigenous $\mathbb{P}^{1}$-bundles on $X^{\mathrm{log}}$. Moreover, the functor that assigns to $T^{\mathrm{log}} \rightarrow S^{\mathrm{log}}$ the set of crystalline Schwarz structures with nilpotent monodromy on $X_{T}^{\mathrm{log}}=X^{\mathrm{log}} \times{ }_{S^{\mathrm{log}}} T^{\mathrm{log}}$ is a torsor over $f_{*}\left(\omega_{X / S}^{\log }\right) \otimes 2(-D)$.

\section{Indigenous Bundles of Restrictable Type}

Let $f_{l}^{\log }: X_{t}^{\log } \rightarrow S^{\log }$ (for $i=1, \ldots, n$ ) be an $r_{i}$-pointed smooth curve of genus $g_{\imath}$ (where $2 g_{\imath}-2+r_{t} \geq 1$ for all $i$ ). Suppose that we are given a graph $\Gamma$ consisting of $n$ vertices, numbered 1 through $n$. Let $E_{\imath}$ be the set of edges of the $i^{\text {th }}$ vertex. Suppose further that we are given an injection $\lambda_{\imath}: E_{\imath} \hookrightarrow\left\{1 \ldots, r_{\imath}\right\}$. Then we can glue together the curves $f_{l}^{\log }: X_{\imath}^{\log } \rightarrow S^{\log }$ to form an $r$-pointed stable curve $f^{\log }: X^{\log } \rightarrow \mathrm{S}^{\log }$ of genus $g$ in such a way that the dual graph of $f^{\text {log }}$ is given by $\Gamma$, that is:

(1) vertex $i$ corresponds to $f_{t}^{\log }: X_{t}^{\log } \rightarrow S^{\log }$, an irreducible component of $X^{\text {log. }}$

(2) if $\epsilon$ is an edge running from vertex $i$ to vertex $j$ such that $\lambda_{\imath}(\epsilon)$ $=a$ and $\lambda,(\epsilon)=b$, then $\epsilon$ corresponds to a node on $X^{\mathrm{log}}$ obtained by gluing together $X_{i}^{\log }$ at the $a^{t h}$ marked point to $X_{j}^{\text {log }}$ at the $b^{t h}$ marked point;

(3) $g$ and $r$ can be computed combinatorially from $\Gamma$, the $g_{i}$ 's, the $r_{\imath}$ 's and the $\lambda$, 's.

Let $\mu_{t}^{\log }: X_{t}^{\log } \hookrightarrow X^{\mathrm{log}}$ be the inclusion of $X_{t}^{\mathrm{log}}$ into $X^{\mathrm{log}}$ as one of the irreducible components.

Now let us suppose that we are given an indigenous bundle $\left(\pi: P \rightarrow X ; \nabla_{P}\right)$ 
on $X^{\text {log }}$. Then it is not necessarily the case that $\left(\mu_{i}^{\text {log }}\right) *\left(\pi: P \rightarrow X ; \nabla_{P}\right)$ will be in digenous on $X_{l}^{\mathrm{log}}$. The problem is that since in general, marked points of $X_{l}^{\text {log }}$ might be sent to nodes of $X^{\text {log }}$ (and not to marked points), there is no reason why the monodromy at such marked points of $X_{\imath}^{\text {log }}$ should be nilpotent. We therefore make the following

Definition 2.10. If the $\left(\mu_{t}^{\text {log }}\right) *\left(\pi: P \rightarrow X ; \nabla_{P}\right)$ are indigenous on $X_{t}^{\text {log }}$ for all $i$, then we say that $\left(\pi: P \rightarrow X ; \nabla_{P}\right)$ is of restrictable type.

Now let us suppose that we are given indigenous bundles $\Pi_{\imath}=\left(\pi_{\imath}: \mathrm{P}_{\imath} \rightarrow\right.$ $\left.X_{i} ; \nabla_{P_{t}}\right)$ on $X_{i}^{\text {log }}$. Note that for each marked point $s: S \rightarrow X_{\imath}$ of an $X_{\imath}^{\text {log }}, s^{*} P_{\imath}$ has a canonical trivialization as a $\mathbb{P}^{1}$-bundle given by considering:

(1) the Hodge section $\sigma_{\imath}: X_{\imath} \rightarrow P_{\imath}$ (pulled back by $s$ );

(2) the trivialization of $s^{*}\left(\sigma_{2}^{*} \omega_{P_{t} / Y_{1}}\right)$ given by the residue map; and

(3) the section $q_{s}: S \rightarrow s^{*} P_{\imath}$ of Proposition 1.6.

It thus follows that we can glue together the $\prod_{1}$ 's by means of this canonical trivialization at the marked points to obtain an indigenous bundle $\mathbb{\Pi}=(\pi: P \rightarrow X$; $\nabla_{P}$ ) on $X^{\mathrm{log}}$. Moreover, by construction, $\Pi$ is of restrictable type.

Also, we can clearly reverse the procedure: Namely, if we start with an indigenous bundle $\mathbb{I}$ on $X^{\mathrm{log}}$ of restrictable type, we can reconstruct $\mathbb{I}$ by restricting to the $X_{i}^{\mathrm{log}}$ s, and then regluing, in the fashion described in the preceding paragraph. Now let us define

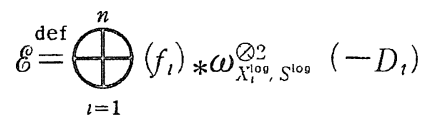

where $D_{\imath}$ is the divisor of marked points on $X_{\imath}$. Then we have the following result:

Proposition 2.11. The étale sheaf on $S$ of isomorphism classes of indigenons $\mathbb{P}^{1}$-bundles of restrictable type on $\mathrm{X}^{\mathrm{log}}$ is a torsor over the vector bundle $\mathscr{E}$.

Note that the rank of $\mathscr{E}$ is given by $\sum_{\imath=1}^{n}\left(3 g_{\imath}-3+r_{\imath}\right)$, which, in general, is strictly less than $3 g-3+r$. 


\section{§3. The Obstruction to Global Intrinsicity}

In $\S 2$, we saw that the $\mathbb{P}^{1}$-bundle $\mathbb{P}\left(\mathscr{g} / \mathscr{g}^{[3]}\right)$ is locally intrinsic on $\bar{M}_{g, r}$. In this Section, we study the obstruction (which, in general, is nonzero) to it being globally intrinsic over all of $\bar{M}_{g, r}$. The main point is a computation in Hodge cohomology which, in many respects, is similar to that of [Falt3], Lemma IV. 4. Since, however, it is not literally the same as [Falt3], and certain technical aspects of the computation are different, we provide a complete proof here.

\section{Introduction of Cohomology Classes}

We shall work over a field $K$ of characteristic zero, say $Q_{p}$, until we state otherwise. Since we are only interested in certain intersection numbers, the base field is essentially irrelevant. Let us consider the universal $r$-pointed stable curve of genus $g, \zeta: \mathscr{C} \rightarrow \bar{M}_{g, r}$ (over $K$ ). We would like to consider various cohomology classes on $\mathscr{C}$ and $\bar{M}_{g, r}$. The cohomology theory that we will use is Hodge theory, so all cohomology classes are to be understood as being Hodge-theoretic. Let $\pi: P=\mathbb{P}\left(\mathscr{F} / \mathscr{J}^{[3]}\right) \rightarrow \mathscr{C}$ be the $\mathbb{P}^{1}$-bundle which, as we saw in $\S 2$. is locally intrinsic. Let $\mathscr{F}=\operatorname{Ad}(P)$. Thus, $\mathscr{F}$ has a filtration whose subquotients are given by:

$$
F^{1} \cong \mathscr{L} ; F^{0} / F^{1} \cong \mathcal{O}_{\mathscr{C}} ; F^{-1} / F^{0} \cong \mathscr{L}^{-1}
$$

where $\mathscr{L}=\omega_{\mathscr{G}^{100 \%} / \bar{H}_{0}^{109} \text {. }}$ Let $\eta=c_{1}(\mathscr{L})$, the first Chern class of $\mathscr{L}$. Then we have: $c_{1}(\mathscr{F})=0$. On the other hand, the second Chern class of $\mathscr{F}$ is given by:

$$
c_{2}(\mathscr{F})=-\eta^{2}
$$

Let us compute $\zeta_{*} \eta^{2}$. Let $D_{i} \subseteq \mathscr{C}$ (where $i=1, \ldots, r$ ) be the marked points. Let $D=\sum_{t=1}^{r} D_{l}$. We shall write $\left[D_{\imath}\right] ;[D]$ for the respective cohomology classes on $\mathscr{C}$. Let $\xi=c_{1}\left(\omega \mathscr{C} / \bar{M}_{o r}\right)$. Thus, $\eta=\xi+[D]$. Since different $D_{i}$ 's do not intersect, we have $\left[D_{l}\right] \cdot\left[D_{j}\right]=0$ if $i \neq j$. Also, by "taking the residue", we see that $\zeta_{*}\left\{\left(\xi+\left[D_{l}\right]\right) \cdot\left[D_{l}\right]\right\}=0$, for all $i$. Thus, $\zeta_{*}\{(\xi+[D]) \cdot[D]\}=0$. Let $\psi_{l}$ $=\zeta_{*}\left(\xi \cdot\left[D_{i}\right]\right) ; \phi=\sum_{i=1}^{r} \phi_{i} ; \theta=\zeta_{*} \xi^{2}$. Then we obtain:

$$
\begin{aligned}
\zeta_{*} \eta^{2} & =\zeta_{*}\{(\xi+[D]) \cdot \xi\} \\
& =\theta+\phi
\end{aligned}
$$


Now one knows from $[\mathrm{AC}]$ that for $g \geq 3$, the restrictions of the classes $\theta$ and $\psi$ to $\mathcal{M}_{g, r}$ are linearly independent. We summarize this in a Lemma:

Lemma 3.1. We have, on $\bar{M}_{g, r}, \zeta * \eta^{2}=\theta+\phi$. In particular, if $g \geq 3$, then $\left.\left(\zeta_{*} \eta^{2}\right)\right|_{\mu_{a}}$ is nonzero.

We shall see below that $\zeta_{* C_{2}}(\mathscr{F})$ can be related to the obstruction to the existence of a global indigenous bundle on $\mathscr{C}$. Thus, once we have done this, we will have proven that this obstruction is given by the relatively computable cohomology class $-\zeta_{*} \eta^{2}$ on $\bar{M}_{g, r}$.

\section{Computation of the Second $\mathbb{C}$ herm $\mathbb{C l a s s}$}

Let us first observe that $\left.P\right|_{D}$ has a canonical trivialization as a $\mathbb{P}^{1}$-bundle given by using:

(1) the Hodge section $\sigma: \mathscr{C} \rightarrow P$ (restricted to $D$ );

(2) the trivialization of $\left.\left(\sigma^{*} \omega_{P / \mathscr{G}}\right)\right|_{D}$ given by the residue map; and

(3) the section $q_{s}: D \rightarrow P \times_{\mathscr{C}} \mathrm{D}$ of Proposition 1.6.

Let us denote by $\mathscr{C}_{\mathrm{c}}^{109}$ the $\log$ stack obtained by letting $\mathscr{C}$ be the underlying stack and taking for the log structure the pull-back of the log structure of $\bar{M}_{g, r}^{\text {log }}$ via $\zeta$. Thus, we have an exact sequence on $\mathscr{C}$ :

$$
0 \rightarrow \zeta^{*} \Omega_{\bar{M}_{q}^{10,},} \rightarrow \Omega_{\mathscr{G}_{c}^{100}} \rightarrow \omega \mathscr{M} / \bar{M}_{q}, \rightarrow 0
$$

where the first two sheaves of differentials are over $K$. In the future, we shall think of this exact sequence as defining a one-step filtration on $\Omega_{G_{c}^{\text {io }}}$. For $i, j \geq$ 0 , let us define for any $\mathscr{O}_{\mathscr{C}}$-module $\mathscr{G}$ :

$$
H_{\mathrm{c}}^{i, J}(\mathscr{C}, \mathscr{G}) \stackrel{\text { det }}{=} H^{2}\left(\mathscr{C}, \mathscr{G} \otimes_{\mathscr{O}_{\mathscr{B}}} \wedge^{\prime} \Omega_{\mathscr{G}_{\mathrm{c}}^{\text {Io }}}\right)
$$

This cohomology is a sort of cohomology with compact supports outside $D$. Thus, by using the canonical trivialization of $\left.P\right|_{D}$ referred to above, we see that the global obstruction to the existence of a logarithmic connection on $\pi: P \rightarrow \mathscr{C}$ (for the $\log$ structure of $\mathscr{C}^{\mathrm{log}}$ ) which has normalized nilpotent monodromy at $D$ defines a class $\kappa \in H_{\mathrm{c}}^{1,1}(\mathscr{C}, \operatorname{Ad}(P))$. Now, by taking the trace of the square of $\kappa$, we get a class $\operatorname{tr}\left(\kappa^{2}\right) \in H_{\mathrm{c}}^{2,2}\left(\mathscr{C}_{0} \mathscr{O}_{\mathscr{C}}\right)$. If we then apply $\zeta_{*}$, we get a class $\zeta_{*} \operatorname{tr}$ $\left(\kappa^{2}\right) \in H^{1,1}\left(\bar{M}_{g, r}^{\text {log }}\right) \stackrel{\text { def }}{=} H^{1}\left(\overline{\mathcal{M}}_{g, r}, \Omega_{\bar{M}_{g r}^{\text {loo }}}^{\text {}}\right)$. On the other hand, let us denote by: 


$$
\overline{\mathscr{S}}_{g, r} \rightarrow \bar{M}_{g, r}
$$

the $\Omega \bar{\mu}_{\theta r}^{\text {loo }}$-torsor defined by looking at the crystalline Schwarz structures with nilpotent monodromy on $\mathscr{C}$ (as in Corollary 2.9). This torsor thus defines a class $\sum \in H^{1,1}\left(\overline{\mathcal{M}}_{g, r}^{\mathrm{log}}\right)$. The goal of this subsection is to prove the following:

Lemma 3.2. We have the following equality of classes in $H^{1,1}\left(\bar{M}_{g, r}^{\mathrm{log}}\right): \Sigma=$ $\frac{1}{2} \zeta_{*} \operatorname{tr}\left(\kappa^{2}\right)$

Now let us note that $H_{\mathrm{c}}^{1,1}(\mathscr{C}, \mathrm{Ad}(P))$ has two one-step filtrations: one arising from the Leray-Serre spectral sequence applied to $\zeta$, and the other arising from the filtration defined above on $\Omega_{G_{c}^{i o g}}$. Thinking in these terms, we see that we get a morphism:

$$
\phi_{00}: H_{c}^{1,1}(\mathscr{C} . \operatorname{Ad}(P)) \rightarrow H^{0}\left(\overline{\boldsymbol{M}}_{g, r}, \mathbb{R}^{1} \zeta_{*}\left(\operatorname{Ad}(P) \otimes \omega_{\mathscr{C}_{1, u_{\theta}}}\right)\right)
$$

Now since we know that $P \rightarrow \mathscr{C}$ admits a connection of the desired type on the fibers of $\zeta$, it follows that $\phi_{00}(\kappa)=0$.

Next let us consider the natural morphism:

$$
\phi_{10}: H_{c}^{1,1}(\mathscr{C}, \operatorname{Ad}(P)) \rightarrow H^{1}\left(\mathscr{C}, \operatorname{Ad}(P) \otimes \omega_{\mathscr{G}, \bar{H}_{\ell},}\right)
$$

Since $\phi_{00}(\kappa)=0$, it follows from considering the Leray-Serre spectral sequence that $\phi_{10}(\kappa)$ lies in $H^{1}\left(\overline{\mathcal{M}}_{g, r}, \zeta_{*}\left(\operatorname{Ad}(P) \otimes \omega_{\mathscr{C}, \overline{\mathcal{M}}_{\theta},}\right)\right) \hookrightarrow H^{1}\left(\mathscr{C}, \operatorname{Ad}(P) \otimes \omega_{\mathscr{C}_{i} ; \overline{\mathcal{M}}_{q}}\right)$. In fact, by considering only normalized connections (as in Definition 1.10), we can say more. Namely, it follows that $\phi_{10}(\kappa)$ is actually the image under the

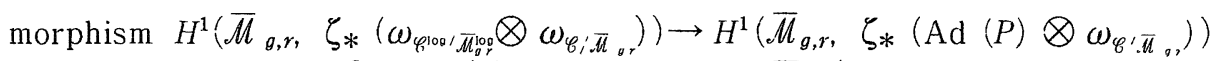

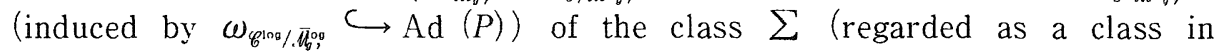

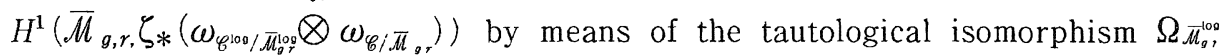
$\left.\cong \zeta_{*}\left(\omega_{\mathscr{G} / 08 / \bar{M}_{g r}^{10},} \otimes \omega_{\mathscr{C} / \bar{M}_{g}}\right)\right)$.

Now let us consider the natural morphism:

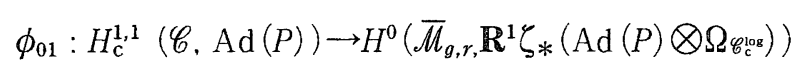

Since $\phi_{00}(\kappa)=0$, it follows that the section $\phi_{01}(\kappa)$ of $\mathbb{R}^{1} \zeta_{*}\left(\operatorname{Ad}(P) \otimes \Omega_{\mathscr{G}_{c}^{\text {Iog }}}\right)$ lies in the image of the natural map 


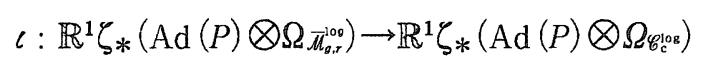

In fact, we can say more. Since we are dealing with sheaves on $\bar{M}_{g, r}$, we can compute locally on $\bar{M}_{g, r}$. Let $U \rightarrow \bar{M}_{g, r}$ be étale. Let $\nabla_{P}$ be a logarithmic connection with normalized nilpotent monodromy (relative to $\zeta_{U}: \mathscr{C}_{U} \rightarrow U$ ) on $P_{U}=$ $P \times \overline{\mathcal{M}}_{g}, U$. Then the obstruction to lifting $\nabla_{P}$ to a logarithmic connection relative to $\mathscr{C}_{U} \rightarrow \operatorname{Spec}(K)$ is giving by subtracting the two pull backs of $\left(P_{U} \rightarrow U\right.$; $\left.\nabla_{P}\right)$ to the first infinitesimal neighborhood $\Delta_{U}$ of the diagonal of $U^{\top} \times{ }_{K} U$. Note that it only makes sense to compare these two pull-backs because we have chosen a connection $\nabla_{P}$, so that we can deal with crystals on Crys $\left(\mathscr{C}_{U} / \Delta_{U}\right)$ (where the structure morphism $\mathscr{C}_{U} \rightarrow \Delta_{U}$ is given by composing $\zeta_{U}: \mathscr{C}_{U} \rightarrow U$ with the diagonal embedding $U \hookrightarrow \Delta_{U}$ ). Thus, the difference between the

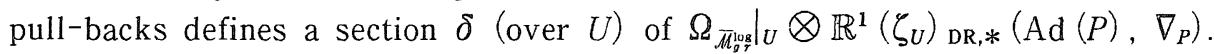
Now if we compose the projection $\mathbb{R}^{1}\left(\zeta_{U}\right)_{\mathrm{DR}, *}\left(\operatorname{Ad}(P), \nabla_{P}\right) \rightarrow \mathbb{R}^{1}\left(\zeta_{U}\right) * \tau_{\mathscr{C}_{U}^{\prime \prime g} / U^{108}}$ with $\delta$, we get a morphism

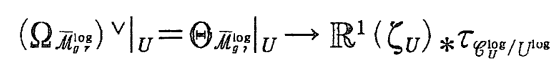

Now it is a lautology that this morphism is none other than the isomorphism $\beta$

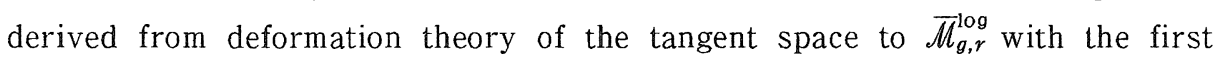
cohomology group of the relative tangent bundle of $\zeta^{\text {log }}$. Thus, in summary, we have proven the following statement:

(*) locally on $\bar{M}_{g, r}, \phi_{10}(\kappa)$ is the image under c of some local section $\nu$

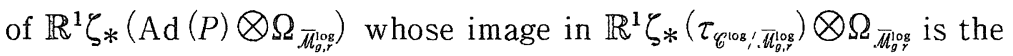
tautological isomorphism $\beta$.

We are now ready to consider $\zeta_{*} \operatorname{tr}\left(\kappa^{2}\right)$. We begin by using the observations of the preceding two paragraphs to compute what happens when we multiply various subquotients of the two filtrations on $H_{\mathrm{c}}^{1,1}(\mathscr{C}, \mathrm{Ad}(P))$ by each other:

(1) If we multiply two elements in the image of $H^{1}\left(\overline{\mathcal{M}}_{g, r}, \zeta_{*}(\operatorname{Ad}(P)) \otimes\right.$

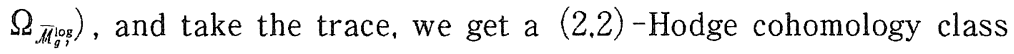
on $\mathscr{C}$ which is the pull-back of such a class on $\bar{M}_{g, r}$, thus, if we apply $\zeta_{*}$ to such a product, we get zero.

(2) If we multiply an element in the image of $H^{1}\left(\bar{M}_{g, r}, \zeta_{*}(\operatorname{Ad}(P)) \otimes\right.$ $\Omega_{\left.\overline{\mathbb{M}}_{g, r, g}\right)}$ by an element in the image of $H^{0}\left(\overline{\mathcal{M}}_{g, r}, \mathbb{R}^{1} \zeta_{*}(\operatorname{Ad}(P)) \otimes\right.$ 
$\Omega_{\left.\bar{M}_{0,0}^{10 g}\right)}$, and take the trace, we get a class in $H^{1}\left(\bar{M}_{g, r}, \mathbf{R}^{1} \zeta_{*} \mathscr{O}_{\mathscr{C}} \otimes\right.$

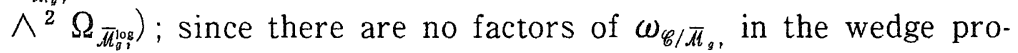
duct, applying $\zeta_{*}$ again gives zero.

(3) If we multiply two elements of $H^{0}\left(\bar{M}_{g, r}, \mathbb{R}^{1} \zeta_{*}(\operatorname{Ad}(P)) \otimes \Omega_{\cdot \bar{u}_{o g}}\right)$, we get zero since $\zeta$ has relative dimension one.

(4) If we multiply an element in the image of $H^{1}\left(\bar{M}_{g, r}, \zeta_{*}(\operatorname{Ad}(P)) \otimes\right.$

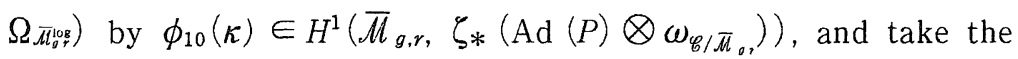
trace, we get zero, since we are taking the trace of the product of a nilpotent section of $\mathrm{Ad}(P)$ with a section of the same Borel subalgebra of $\operatorname{Ad}(P)$.

(5) If we square $\phi_{10}(\kappa) \in H^{1}\left(\bar{M}_{g, r}, \zeta_{*}\left(\operatorname{Ad}(P) \otimes \omega_{\mathscr{C} / \bar{M}_{g}}\right)\right)$, we get zero since we are, in effect, squaring nilpotent sections of $\operatorname{Ad}(P)$.

(6) If we multiply $\phi_{10}(\kappa) \in H^{1}\left(\bar{M}_{g, r}, \zeta_{*}\left(\operatorname{Ad}(P) \otimes \omega_{\mathscr{G} / / \bar{M}_{\bullet}}\right)\right)$ by $\phi_{01}(\kappa) \in$ $H^{0}\left(\bar{M}_{g, r}, \mathbb{R}^{1} \zeta_{*}\left(\operatorname{Ad}(P) \otimes \Omega_{\mathscr{q}_{c}^{108}}\right)\right)$, then we are, in effect, multiplying the class $\sum$ by the tautological isomorphism $\beta$. so that we obtain $\sum$, regarded as a class in $H^{1}\left(\bar{M}_{g, r}, \Omega{\overline{M_{g r}}}_{r}\right)$.

Thus, in summary, all of the possible contributions are zero. except for the last, which is $2 \sum$. This completes the proof of Lemma 3.2. On the other hand, by basic linear algebra, $c_{2}(\mathrm{Ad}(P))$ is $-2 \operatorname{tr}\left(\kappa^{2}\right)$, so we see that we have, in fact, proven the following;

Lemma 3.3. We have $\zeta_{* c_{2}}(\operatorname{Ad}(P))=-4 \sum$.

Finally, putting this together with Lemma 3.1. we see that we have explicitly computed the class $\sum$ in terms of well-known first Chern classes of line bundles:

Theorem 3.4. The torsor of Schwarz structures defines a class $\sum \in H^{1}\left(\bar{M}_{g, r}\right.$. $\left.\Omega_{\bar{M}_{\xi}^{\text {log }}}\right)$ which is equal to $\frac{1}{4}(\theta+\phi)$, where $\theta=\zeta_{*} \xi^{2} ; \phi=\zeta_{*}(\xi \cdot[D]) ; \xi=c_{1}\left(\omega_{\mathscr{C} / \overline{\mathcal{M}}_{\theta}}\right)$; and $[D]$ is the cohomology class of the divisor of marked points. In particular, if $g \geq 3$, then (in characteristic zero) $\mathscr{\&}_{g, r} \rightarrow M_{g, r}$ does not admit any sections, i.e., there are no canonical Schwarz structures on r-pointed smooth curves of genus $g \geq 3$.

Remark. Ideally, it would be nice to have an equality of classes not in 
$H^{1}\left(\bar{M}_{g, r}, \Omega_{\bar{M}_{g r}^{\circ g}}\right)$, but in some sort of cohomology with compact supports " $H_{\mathrm{c}}^{1}\left(\overline{\mathcal{M}}_{g, r}\right.$, $\left.\Omega_{\bar{M}_{g} \text { igg }}\right)$ " (which should be isomorphic to $H^{1}\left(\bar{M}_{g, r}, \Omega_{\bar{M}_{g, r}}\right)$ ). In order to do this, one would have to define some sort of appropriate sense in which $\Sigma$ is compactly supported, i.e., one would have to define some sort of trivializations of $\bar{\varnothing}_{g, r} \rightarrow$ $\bar{M}_{g, r}$ at infinity. In fact, $\overline{\mathscr{\Phi}}_{g, r} \rightarrow \bar{M}_{g, r}$ does not (in general) have a canonical section over the divisor at infinity. However, by considering indigenous bundles of restrictable type, one can show that, so to speak, "the more singular a curve gets, the more of a canonical trivialization one has for $\overline{\mathscr{S}}_{g, r} \rightarrow \bar{M}_{g, r}$." For instance, if the curve is totally degenerate, i.e., it can be constructed by gluing together (as at the end of §2) a number of copies of $\mathbb{P}^{1}$ with three marked points, then $\bar{\varnothing}_{g, r} \rightarrow$ $\bar{M}_{g, r}$ does have a canonical trivialization, as follows immediately from Proposition 2.11 (since then the indigenous bundles of restrictable type form a torsor over the zero sheaf). Thus, in some sort of combinatorially complicated sense, by considering indigenous bundles of restrictable type, one can exhibit $\sum$ as a cohomology class with compact supports. Unfortunately, however, the combinatorics involved get rather complicated in general, so we shall not carry this out explicitly, except in the case when the dimension of $\bar{M}_{g, r}$ is one, where things are not so difficult.

\section{The Case of $\mathbb{D}$ imension One}

In this case, either $g=r=1$ or $g=0, r=4$. Let $D_{\infty} \subseteq \bar{M}_{g, r}$ be the divisor at infinity. Since $D_{\infty}$ is zero-dimensional, in this case we do have a canonical trivialization $t_{\infty}$ of $\bar{\bigotimes}_{g, r} \rightarrow \bar{M}_{g, r}$ over $D_{\infty}$. Now we shall give a new definition of cohomology with compact supports that takes into account this trivialization $t_{\infty}$.

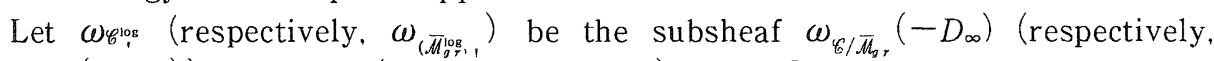

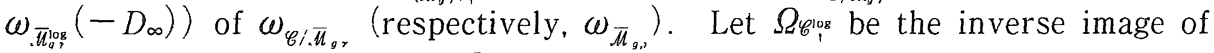

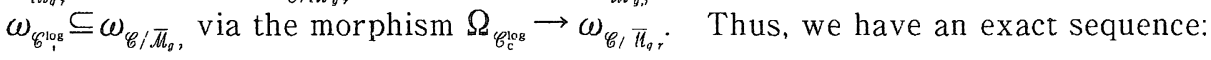

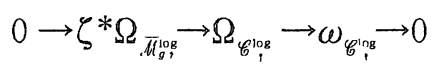

which defines a filtration on $\Omega_{\mathscr{G} \text { 'og. }}$. Then for $i, j \geq 0$, let us define for any $B_{\mathscr{C}}$-module $\mathscr{G}$ :

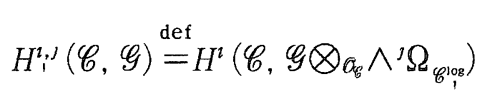

and for any $\mathscr{O}_{\bar{M}_{g}}$-module $\mathscr{H}$ :

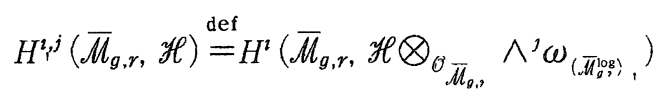


Note that the push forward map $\zeta_{*}$ naturally defines a morphism $\zeta_{*}: H^{2,2}(\mathscr{C}$, $\left.\mathscr{O}_{\mathscr{C}}\right) \rightarrow H^{1,1}\left(\overline{\mathcal{M}}_{g, r}, \mathscr{O}_{\bar{M}_{\theta}}\right)$. Now the obstruction to putting a logarithmic connection on $P \rightarrow \mathscr{C}$ with normalized nilpotent monodromy at the marked points and which is of restrictable type at infinity is given by a class $\kappa ! \in H^{1,1}(\mathscr{C}, \operatorname{Ad}(P))$. On the other hand, the torsor $\bar{\varnothing}_{g, r} \rightarrow \bar{M}_{g, r}$ together with the trivialization $t_{\infty}$ defines a class $\sum_{!} \in H^{1,1}\left(\bar{M}_{g, r}, \mathcal{O}_{\bar{M}_{0}}\right)$.

By the same proof as before, we have $\Sigma_{1}=\frac{1}{2} \zeta_{*} \operatorname{tr}\left(\kappa_{1}^{2}\right)$. (In the present case, however, one might remark that in the six types of product considered previously, the first two types (numbered (1) and (2)) of product vanish all the more trivially since they involve $\wedge^{2} \omega_{\overline{\bar{A}_{g}^{\text {og }}}}$, which is zero.) Also, just as before, we have $c_{2}(\operatorname{Ad}(P))=-2 \operatorname{tr}\left(\kappa_{1}^{2}\right)$. Thus, we obtain:

Theorem 3.5. In $H^{1,1}\left(\bar{M}_{g, r}, \mathscr{O}_{\bar{M}_{n r}}\right)=K$, we have

$$
\Sigma_{1}=\frac{1}{4} \zeta_{*} \eta^{2}
$$

where $\eta=c_{1}\left(\omega_{\mathscr{G}^{108} / \bar{M}_{9}^{108}}\right)$.

Let us compute $\zeta_{*} \eta^{2}$ in the case $g=1, r=1$. First we introduce the classes $\theta$ $=\zeta_{*} \xi^{2} ; \phi=\zeta_{*}(\xi \cdot[D])$; and $\lambda={ }_{c_{1}}\left(\zeta_{*} \omega_{\mathscr{C}_{i} / \bar{M}_{\theta_{1}}}\right)$. By Grothendieck-Riemann-Roch, $\theta$ $=0$. On the other hand, sorting through the definitions, one sees that $\phi=\lambda$. Thus, we obtain that for $g=1, r=1$ :

$$
\Sigma_{1}=\frac{1}{4} \lambda
$$

Next let $N^{\log :} \bar{M}_{1,1}^{\log }[2] \rightarrow \bar{M}_{1,1}^{\log }$ be the finite, log étale covering such that $\bar{M}_{1,1}^{\log }$ [2] is the moduli stack that parametrizes elliptic curves with level structure on the 2 -torsion points. Let

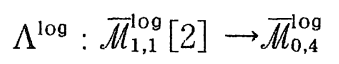

be the log étale morphism given by sending an elliptic curve with a trivialization of its two torsion to the four-pointed curve of genus zero of which the elliptic curve is a double covering (with ramification exactly at the four marked points). Moreover, $\Lambda^{\log }$ admits a section over any double covering of $\bar{M}_{0,4}^{\log }$ (since the obstruction to such a section lies in $H_{\text {et }}^{2}\left(\bar{M}_{0,4}, \mathbf{Z} / 2 \mathbf{Z}\right)$ ). Note 
that both $N^{\log }$ and $\Lambda^{\log }$ are, in fact, defined over $\mathbb{Z}\left[\frac{1}{2}\right]$. Also, note that (over $\mathbb{Z}\left[\frac{1}{2}\right]$ ), we have an isomorphism $\Lambda^{*} \bar{\varnothing}_{0,4} \cong N^{*} \bar{\varnothing}_{1,1}$ obtained by pulling-back and pushing forward indigenous bundles. We thus obtain the following result:

Theorem 3.6. When $\bar{M}_{g, r}$ is one-dimensional, the torsor $\overline{\mathscr{\bigotimes}}_{g, r} \rightarrow \bar{M}_{g, r}$ does not admit any section which is equal to $t_{\infty}$ over $D_{\infty}$ modulo any prime $\geq 5$.

Proof. We have $\left(\sum !\right)_{1,1}=\frac{1}{4} \lambda_{1,1}$, and $\lambda_{1,1}=\frac{1}{2} c_{1}\left(\omega_{\overline{\mathcal{M}_{1:}^{10 P}}}\right)$, so $\left(\sum !\right)_{1,1}=$ $\frac{1}{8} c_{1}\left(\omega_{\overline{\mathcal{M}_{1}^{\text {lop }}}}\right)$. Thus, since $N^{\mathrm{log}}$ is log étale, and $N^{*}\left(\Sigma_{!}\right)_{1,1}$ is an invertible multiple of $\Lambda^{*}\left(\Sigma_{!}\right)_{0,4}$, it suffices to show that:

(1) in Hodge cohomology modulo $p$ (for a prime $p \geq 5), \Lambda^{*} c_{1}\left(\omega_{\bar{M}_{08}^{\circ 0}}\right)=$ $N^{*} c_{1}\left(\omega_{\overline{M_{10}^{\circ P}}}\right)$ is nonzero; in fact, since $\Lambda^{\text {log }}$ admits a section over any double covering of $\overline{\mathcal{M}}_{0,4}^{\log }$, it suffices merely to show that $c_{1}\left(\omega_{\overline{\mathcal{M}}_{0.4}^{+o g}}\right)$ is nonzero modulo $p$;

(2) the formation of $H_{1}^{1,1}\left(\bar{M}_{0,4}, \mathscr{O}_{\bar{M}_{04}}\right)$ commutes with base change modulo $p$ (for $p$ an odd prime).

But both (1) and (2) follow immediately from the fact that $\bar{M}_{0,4}$ is just $\mathbb{P}^{1}$, with $D_{\infty}=\{0,1, \infty\}$. (Note that there is a slight subtlety here in that (2) is not immediately obvious for $\bar{M}_{1,1}$ since it is a stack; this is why we choose to verify the assertions of the Theorem by means of $\bar{M}_{0,4}$.)

\section{Appendix: Relation to the Complex Analytic Case}

In this Appendix, we make the connection between the theory of Schwarz structures discussed here and the classical notion of the Schwarzian derivative in complex analysis. Let $K$ be an algebraically closed field of characteristic zero (such as, for instance, the complex numbers $\mathbb{C}$ ). Let $X \rightarrow \operatorname{Spec}(K)$ be a smooth, proper, connected curve. Let $P=\mathbb{P}\left(\mathscr{g} / \mathscr{g}^{[3]}\right)$ be the usual $\mathbb{P}^{1}$-bundle on $X$, and let $\nabla_{P}$ be a connection on $P \rightarrow X$ that makes it indigenous. Then just as in $\S 1$, we can form the Schwarzian derivative:

$$
D: \mathscr{O}_{X}^{\#} \rightarrow \omega_{X / K}^{2}
$$


Then the purpose of this Appendix is to show that when $X=\mathbf{P}^{1} ; z$ is the standard rational function on $\mathbb{P}^{1}$; and $\omega_{X / K}^{2}$ is trivialized by $(d z)^{2}$, then $D(\phi)$ is given (up to a factor of two) by the classical formula for the Schwarzian derivative.

First, let us note that when $X=\mathbf{P}^{1}$, there exists only one connection $\nabla_{P}$ on $\pi: P \rightarrow X$ that makes it indigenous. Indeed, this follows from the fact that $\operatorname{Ad}(P) \otimes \sigma_{x} \omega_{X / K}$ has no sections for degree reasons, plus the fact that the extension

$$
0 \rightarrow \mathscr{g}^{[2]} / \mathscr{g}^{[3]} \rightarrow \mathscr{g} / \mathscr{g}^{[3]} \rightarrow \mathscr{g} / \mathscr{g}^{[2]} \rightarrow 0
$$

does not split (since the extension class is the Hodge-theoretic first Chern class $c_{1}\left(\omega_{X / K}\right)$, which is nonzero $)$. It thus follows that $\left(P: \nabla_{P}\right)$ is necessarily isomorphic to the indigenous bundle constructed in Example 1 of $\S 2$. Thus, $P \cong X \times_{K} X$ (where we regard the projection to the second factor as the structure morphism to $X$ ) : let us fix such an isomorphism for the rest of the discussion. Also, the Kodaira-Spencer morphism at the diagonal section $\sigma_{\Delta}: X \rightarrow X \times{ }_{K} \mathrm{X} \cong P$ is an isomorphism. Let $z$ be the standard rational function on $X$. Let $U \subseteq X$ be the complement of infinity. Thus, $z$ is regular on $U$. We shall work mainly on $U$. For $i=1,2$, let $p_{\imath}: P \cong X \times_{K} X \rightarrow X$ be the projections to the $i^{t h}$ factor. Let $\zeta$ be the relative rational function on $\left.P\right|_{U} \rightarrow U$ given by $p_{1}^{*}(z)-p_{2}^{*}(z)$. Let us simply denote by $\nabla$ the result of applying $\nabla_{P}$ in the tangent direction $\frac{d}{d z}$. Then clearly, $\nabla(\zeta)=-1$. Let $\eta=1 / \zeta$. Thus, $\nabla(\eta)=\eta^{2}$. Now if we regard $P$ as $\mathbb{P}$ $\left(\mathscr{g} / \mathscr{g}^{[3]}\right)$, and $s$ is a section given by $\left[a d z, b(d z)^{2}\right]$ (where $a, b \in \mathscr{O}_{X}(V)$, for some open $V \subseteq U)$, then we have $\zeta(s)=a / b$. Indeed, both sides of this equation define relative rational functions on $\left.P\right|_{U} \rightarrow U$. The right-hand side has a simple pole at the section $\left[d z, 0 \cdot(d z)^{2}\right]$, which, by computing residues as in the definition of the isomorphism of Proposition 1.4, corresponds to the section $\infty \times U$. Thus, both sides of the equation have a simple pole at $\infty \times U$, and the same 1 -jet at $\sigma_{\Delta}$, hence are equal. In particular, $\eta(s)=b / a$.

Now suppose we are given $\phi \in \mathscr{O}_{X}^{\#}(V)$. The 2-jet $j_{\phi}$ of $\phi$ is given by $\phi^{\prime} d z$ $+\frac{1}{2} \phi^{\prime \prime}(d z)^{2}$. Thus, if $s_{\phi}$ is the section of $P$ over $V$ that is defined by $j_{\phi}$, we have $\eta\left(s_{\phi}\right)=\frac{\phi^{\prime \prime}}{2 \phi^{\prime}}$. Thus, we compute: 


$$
\begin{aligned}
\left(\frac{\phi^{\prime \prime}}{2 \phi^{\prime}}\right)^{\prime} & =\nabla\left(\eta\left(s_{\phi}\right)\right) \\
& =\{\nabla(\eta)\}\left(s_{\phi}\right)+\eta\left(\nabla\left(s_{\phi}\right)\right) \\
& =\eta^{2}\left(s_{\phi}\right)+D(\phi)(d z)^{-2} \\
& =\frac{\left(\phi^{\prime \prime}\right)^{2}}{4\left(\phi^{\prime}\right)^{2}}+D(\phi)(d z)^{-2}
\end{aligned}
$$

Expanding the derivative on the left and rearranging terms, we get:

$$
\begin{aligned}
D(\phi) & =\left\{\frac{\phi^{\prime \prime \prime}}{2 \phi^{\prime}}-\frac{\left(\phi^{\prime \prime}\right)^{2}}{2\left(\phi^{\prime}\right)^{2}}-\frac{\left(\phi^{\prime \prime}\right)^{2}}{4\left(\phi^{\prime}\right)^{2}}\right\}(d z)^{2} \\
& =\frac{1}{2}\left\{\frac{\phi^{\prime \prime \prime}}{\phi^{\prime}}-\frac{3\left(\phi^{\prime \prime}\right)^{2}}{2\left(\phi^{\prime}\right)^{2}}\right\}(d z)^{2}
\end{aligned}
$$

We have thus shown the following result:

Theorem $\mathbb{A}$. On $\mathbb{P}^{1}$, the Schwarzian derivative defined at the end of $\S 1$ is equal to one-half the classical Schwarzian derivative.

For a treatment of the classical Schwarzian derivative, we refer to [Lehto].

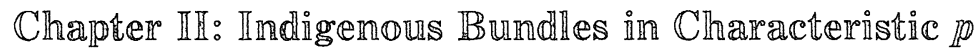

\section{\$0. Introduction}

In this Chapter, we study indigenous bundles in characteristic $p$. In particular, we will be concerned with how these bundles interact with Frobenius. Our main tool for studying this interaction will be the p-curvature. We begin in $\S 1$ by studying FL-bundles, which are a special kind of rank two vector bundle with connection on a curve that corresponds to a lifting of the curve modulo $p^{2}$. In $\S 2$, we define the Verschiebung map on indigenous bundles to be the determinant of the $p$-curvature of the indigenous bundle. It turns out that (essentially) indigenous bundles arise from FL-bundles precisely when their Verschiebung vanishes. Since it is precisely this sort of indigenous bundle which (following $[\mathrm{Katz}]$ ) we call nilpotent - that corresponds to an $\mathcal{M F}^{\nabla}$ -object in the sense of [Falt]. it is worthwhile defining and studying the moduli 
space $\overline{\mathcal{N}}_{g, r}$ of such bundles. In order to study $\overline{\mathcal{N}}_{g, r}$, we make two fundamental calculations (Theorems 2.3 and 2.13) concerning the Verschiebung. The first tells us that the Verschiebung is finite and flat, of degree $p^{3 g-3+r}$, and the second calculates the derivative the Verschiebung in terms of invariants of the indigenous bundle which are easier to compute. In $\S 3$, we define the hyperbolic (higher genus) analogue of an ordinary elliptic curve: namely, we say that a hyperbolic curve is hyperbolically ordinary if it admits a nilpotent indigenous bundle at which the derivative of the Verschiebung is an isomorphism. Using the general machinery developed in $\$ 2$, we then do a number of computations involving totally degenerate curves and elliptic curves which reveal that:

(1) the hyperbolically ordinary locus of $\bar{M}_{g, r}$ is open and dense (Corollary 3.8);

(2) if one applies the definition of ordinariness in terms of indigenous bundles to the case of elliptic curves, one recovers the classical definition of an ordinary elliptic curve (Theorem 3.11); and

(3) (at least if $g \geq 3$, and $p$ is sufficiently large then) each irreducible component of $\overline{\mathcal{N}}_{g, r}$ that passes through a certain canonical nilpotent indigenous bundle on a totally degenerate curve has degree $\geq 2$ over $\bar{M}_{g, r}$; thus, there is no canonical choice of a nilpotent indigenous bundle on a generic $r$-pointed stable curve of genus $g$ (Proposition 3.13).

We end the Chapter with the observation that (3) is interesting in the sense that it constitutes a deviation from the behavior that one might expect by analogy to the complex case.

\section{$\S 1 . \quad F L$ Bundles}

In this Section. we develop the theory of a certain kind of rank two bundle, which we call an FL-bundle, which arises from looking at the Cartier isomorphism of a curve. It turns out that space of such bundles can also be used to parametrize the infinitesimal deformations of a curve to $\mathbb{Z} / p^{2} \mathbf{Z}$. The material we present here is essentially "well-known" (see, e.g., [Kato], §4), although our point of view is a little different. Let $p$ be a prime. Let $S$ be a noetherian scheme over $\mathbf{F}_{p}$. Let us assume that we are given a fine $\log$ structure on $S$, and let us denote the resulting $\log$ scheme by $S^{\log }$. Let us denote the absolute Frobenius ([Kato], §4) of $S^{\log }$ by $\Phi_{S^{\log }}: S^{\log } \rightarrow S^{\log }$. Let $f^{\log }: X^{\log } \rightarrow S^{\log }$ be an $r$-pointed stable curve of genus $g$ (as in Chapter I, Definition 2.1, so $2 g-2+r \geq$ 1 ). In general, we shall denote by means of a superscript " $F$ " the result of 
base-changing by $\Phi_{S^{\log 8}}$ Let $\Phi_{X^{\log 8} / S^{\log }}: X^{\log } \rightarrow\left(X^{\log }\right) F \stackrel{\text { def }}{=} X^{\log } \times_{S^{\log , \Phi_{S^{108}}}} S^{\log }$ be the relative Frobenius.

\section{Deformations and $\mathbb{F L}$-Bundles}

We begin by reviewing the Cartier isomorphism (as in [Kato], Theorem 4.12). Since a curve is one-dimensional, this amounts to the existence of an exact sequence of sheaves on $X$ :

$$
0 \rightarrow\left(\Phi_{X^{108} / S^{108}}\right)^{-1} \mathscr{O}_{X^{F}} \rightarrow \mathscr{O}_{X} \rightarrow \omega_{X / S}^{\log } \rightarrow\left(\Phi_{X^{108} / S^{108}}\right)^{-1}\left(\omega_{X / S}^{\log }\right)^{F} \rightarrow 0
$$

where the morphism is the middle is the exterior differentiation operator $d$. Let $\mathscr{Q} \stackrel{\text { def }}{=} d\left(\mathscr{O}_{X}\right) \subseteq \omega_{X / S}^{\log }$. Then note that since the above exact sequence is functorial with to base-change $T^{\log } \rightarrow S^{\log }$, the formation of $\mathscr{Q}$ is likewise functorial with respect to base-change. We would like to consider what happens to this exact sequence when it is tensored over $\left(\Phi_{X^{108} / S^{108}}\right)^{-1} \mathscr{O}_{X^{F}}$ with $\left(\Phi_{X^{108} / S^{108}}\right)^{-1}$ $\left(\tau_{Y^{108} / S^{108}}\right)^{F}$. Let $\mathscr{T}=\left(\Phi_{X^{108} / S^{108}}\right)^{*}\left(\tau_{X^{118} / S^{108}}\right)^{F}$. We then obtain (by using the long exact cohomology sequence for higher direct images) the following two exact sequences of sheaves on $S$ :

$$
\begin{gathered}
0 \rightarrow \mathscr{O}_{S} \rightarrow \mathbb{R}^{1} f_{*} \mathscr{Q} \otimes_{\mathscr{O}_{x}} \mathscr{T} \rightarrow \mathbb{R}^{1} f_{*} \omega_{X / S}^{\log } \otimes_{\mathfrak{C}_{x}} \mathscr{T} \\
0 \rightarrow \mathbb{R}^{1} f_{*}\left(\tau_{\left.\lambda^{\log / S^{\log }}\right)^{F} \rightarrow \mathbb{R}^{1} f_{*} \mathscr{T}}^{\rightarrow} \mathbb{R}_{1} f_{*} \mathscr{Q} \otimes_{\mathcal{C}_{\mathrm{r}}} \mathscr{T} \rightarrow 0\right.
\end{gathered}
$$

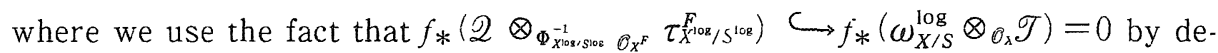
gree considerations. Now let us note that $\mathscr{T}$ has a natural logarithmic connec-

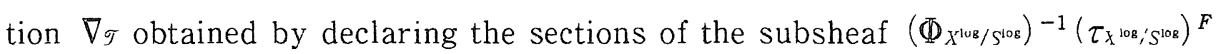
$\subseteq\left(\Phi_{\lambda^{108} / S^{108}}\right) *\left(\tau_{X^{108} / S^{108}}\right) F=\mathscr{T}$ to be horizontal. Thus, by using the above exact sequences, we can compute the first de Rham cohomology module of $\mathscr{T}$ (where we always understand $\mathscr{T}$ to be equipped with the connection $\nabla_{\mathscr{T}}$ ).

Proposition 1.1. We have an exact sequence:

$$
0 \rightarrow \mathbb{R}^{1} f_{*}\left(\tau_{X^{108} / S^{108}}\right)^{F} \rightarrow \mathbb{R}^{1} f_{D R, *}(\mathscr{T}) \rightarrow \mathscr{O}_{S} \rightarrow 0
$$

which is functorial with respect to base-change $T^{\log } \rightarrow S^{\log }$. In particular, $\mathbb{R}^{1} f_{D R, *}$ $(\mathscr{T})$ is a vector bundle of rank $3 g-2+r$ on $S$. Finally, $\mathbb{R}^{1} f_{\mathrm{DR}, *}(\mathscr{T}) \subseteq \mathbb{R}^{1} f_{*}(\mathscr{T})$.

Let us denote by $\mathscr{A}$ the $\mathbb{R}^{1} f_{*}\left(\tau_{X^{\mathrm{IOg} / S^{\mathrm{IO}}}}\right)^{F}$-torsor on $S$ defined by the above exact sequence. Let $\widetilde{S}^{\log }$ be a fine $\log$ scheme whose underlying scheme is flat

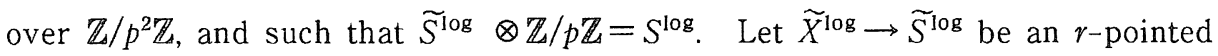


stable curve of genus $g$ lifting $f^{\text {log }}$. Then for any $r$-pointed stable curve $\widetilde{Y}^{\text {log }} \rightarrow$ $\widetilde{S}^{\log }$ of genus $g$ that lifts $\left(X^{\log }\right)^{F} \rightarrow S^{\log }$, we can associate a section $\theta_{Y}$ of $\mathscr{A}$ as fol-

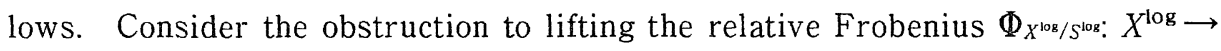
$\left(X^{\log )^{F}}\right.$ to a morphism $\widetilde{X}^{\log } \rightarrow \widetilde{Y}^{\log }$. This defines a section $\theta_{Y}^{\prime}$ of $\mathbf{R}^{1} f_{*}(\mathscr{T})$. Observe that $\theta_{Y}^{\prime}$ is independent of the choice of lifting $\widetilde{X}^{\mathrm{log}}$. Indeed, this follows from the fact that locally, if one changes the lifting $\widetilde{X}^{10 g}$, the obstruction cocycle will change by a derivation of $X^{\log }$ applied to a function pulled-back via $\Phi_{X^{108} / S^{108}}$ from $\left(X^{\mathrm{log}}\right)^{F}$. But this will always give zero. This proves that $\theta_{Y}^{\prime}$ depends only on $\widetilde{Y}^{\text {log }}$. Let us also observe that $\theta_{Y}^{\prime}$ actually defines a section of $\mathbf{R}^{1} f_{D R, *}(\mathscr{T})$ $\subseteq \mathbb{R}^{1} f_{*}(\mathscr{T})$. Indeed, to see this, we reason as follows. We work with bianalytic functions, as in Chapter I, $\S 1$. Then the inverse image via the relative Frobenius of $\mathscr{O}_{\left(x^{b_{1}}\right)^{F}}$ in $\mathscr{O}_{\mathscr{C}^{b_{2}}}$ coincides with both $i_{\mathrm{L}}\left\{\left(\Phi_{\left.\chi^{\mathrm{log}} / S^{\mathrm{jog}}\right)}{ }^{-1} \mathscr{O}_{\mathscr{Y}_{F} F}\right\}\right.$ and

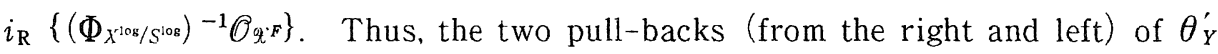
to $\mathscr{O}_{\tilde{X}^{b i}}$ both correspond to the obstruction to lifting $i_{\mathrm{L}}\left\{\left(\Phi_{X^{108} / S^{108}}\right)^{-1} \mathscr{O}_{\mathscr{X}_{F}}\right\}=$ $i_{R}\left\{\left(\Phi_{X^{10 z} / S^{108}}\right)^{-1} \mathscr{O}_{x^{F} F}\right\}$ to an $\mathscr{O}_{\tilde{S}}$-flat subalgebra (with log structure) of $\mathscr{O}_{\tilde{\mathscr{X}}}$. Th. This shows that $\theta_{Y}^{\prime}$ is horizontal.

Now suppose that we consider another lifting $\widetilde{Z}^{\text {log }} \rightarrow \widetilde{S}^{\log }$ of $\left(X^{\log }\right)^{F} \rightarrow S^{\text {log }}$. Then the difference between the liftings $\widetilde{Y}^{\text {Tog }}$ and $\widetilde{Z}^{\text {log }}$ naturally defines a section $\theta_{Y Z}$ of the vector bundle $\mathbb{R}^{1} f_{*}\left(\tau_{\left.X^{\log / S^{\ln }}\right)}\right)^{F}$. It follows immediately from the definition of an obstruction class that $\theta_{Y}^{\prime}=\theta_{Z}^{\prime}+\theta_{Y Z}$. Thus, if there existed a lifting $\widetilde{Y}^{\text {log }}$ such that $\theta_{Y}^{\prime}$ is a section of the subsheaf $\mathbb{R}^{1} f_{*}\left(\tau_{X^{108} / S^{108}}\right)^{F} \subseteq \mathbf{R}^{1} f_{\mathrm{DR}, *}(\mathscr{T})$, then it would follow that there exists another lifting $\widetilde{Z}^{\log }$ such that $\theta_{Z}^{\prime}=0$. Thus, the relative Frobenius would lift to a morphism $\Psi: \widetilde{X}^{\log } \rightarrow \widetilde{Z}^{\log }$. But then by pull-back, $\Psi$ would induce a nonzero morphism of $\left(\Phi_{\left.\mathrm{Y}^{\mathrm{jog} /} / \mathrm{S}^{\mathrm{log}}\right)} *\left(\omega_{X / S}^{\log }\right)^{F}\right.$ into $\omega_{X / S}^{\log }$, which, by degree considerations, is absurd. Thus, we conclude that no $\theta_{Y}^{\prime}$ lies in $\mathbf{R}^{1} f_{*}\left(\tau_{X^{108} / S^{108}}\right)^{F} \subseteq \mathbf{R}^{1} f_{\mathrm{DR}, *}(\mathscr{T})$. In other words, for every lifting $\widetilde{Y}^{\log }, \theta_{Y}$ defines a section of $\mathscr{A}$. Let $\mathscr{D}$ be the $\mathbb{R}^{1} f_{*}\left(\tau_{\left.\chi^{\log } / S^{\log }\right)}\right)^{F}$-torsor over $S$ of liftings of $\left(X^{\log }\right)^{F} \rightarrow S^{\log }$ to $\widetilde{S}^{\log }$. Then, we see that wa have defined a canonical morphism of $\mathbb{R}^{1} f_{*}\left(\tau_{\left.\lambda^{\log } / S^{\log }\right)}^{F}\right.$-torsors

$$
\mathscr{F}: \mathscr{D} \rightarrow \mathscr{A}
$$

Since any morphism of torsors is necessarily an isomorphism, we see that we have proven the following result: ([Kato], Theorem $4.12(2)$ )

Proposition 1.2. The canonical morphism $\mathscr{F}: \mathscr{D} \rightarrow \mathscr{A l}$ is an isomorphism.

Let $\left(\mathscr{E}, \nabla_{\mathscr{E}}\right)$ be a rank two vector bundle on $X$ with a connection $\nabla_{\mathscr{E}}$ 
(relative to $f^{\mathrm{log}}$ ). Suppose that there exists a horizontal exact sequence

$$
0 \rightarrow \mathscr{T} \rightarrow \mathscr{E} \rightarrow \mathscr{O}_{X} \rightarrow 0
$$

Then this exact sequence defines a section $\eta$ of $\mathbb{R}^{1} f_{\mathrm{DR}, *}(\mathscr{T})$ over $S$.

Defininition 1 .. We shall call $\left(\mathscr{E}, \nabla_{\mathscr{E}}\right)$ an $\mathrm{FL}$ - (vector) bundle if $\eta$ maps to $\mathscr{O}_{S}^{\times} \subseteq \mathscr{O}_{S}$ under the map $\mathbb{R}^{1} f_{\mathrm{DR}, *}(\mathscr{T}) \rightarrow \mathscr{O}_{S}$ of Proposition 1.1. We shall call a $\mathbb{P}^{1}$-bundle with connection $\left(P, \nabla_{P}\right)$ on $X^{\log }$ an FL- $\left(\mathbb{P}^{1}\right)$-bundle if it can be written étale locally on $S$ as the $\mathbb{P}^{1}$-bundle associated to an $\mathrm{FL}$-vector bundle.

Remark. The letters "FL" stand for Frobenius lifting. Since an FL-bundle defines a section of the torsor $\mathscr{A}$, it follows by Proposition 1.2 that it also denines a lifting $\widetilde{Y}^{\log } \rightarrow \widetilde{S}^{\text {log }}$ of $\left(X^{\log }\right)^{F} \rightarrow S^{\log }$. Also, we shall see below (Corollary 1.5) that, at least if $S$ is reduced, then if a horizontal exact sequence as above exists, it is necessarily unique.

\section{The $p$-Curvature of an $\mathbb{F}-\mathbb{B}$ undle}

Let us assume for the rest of the Section that $p$ is odd. Throughout this Chapter the notion of the $p$-curvature of a bundle with connection in characteristic $p$ will play an important role. We refer to [Katz]. §5, 6 ; [Ogus], §1.2, 1.3 for basic facts concerning the $p$-curvature. ([Katz]. of course, does not handle the arbitrary "log-smooth" case, but the definitions and proofs (of the properties that we will need) go through without change. At any rate, on the sorts of curves that we are working with, the theory of [Katz], §5.6, is literally valid on an open, schematically dense subset, and many assertions can be checked after restriction to such an open subset.) Let $\left(\mathscr{E}, \nabla_{\mathscr{E}}\right)$ be an FL-bundle. We would like to compute the $p$-curvature of $\left(\mathscr{E}, \nabla_{\mathscr{E}}\right)$. The $p$-curvature $\mathscr{P}$ will be a horizontal section of $\mathscr{T}^{\vee} \otimes_{\mathscr{O}_{\lambda}}$ Ad $(\mathscr{E})$. Occasionally, we shall think of $\mathscr{P}$ as a morphism $\operatorname{Ad}(\mathscr{E}) \rightarrow \mathscr{T}^{\vee}$ or a morphism $\mathscr{T} \rightarrow \operatorname{Ad}(\mathscr{E})$ (using the fact that $\operatorname{Ad}(\mathscr{E})$ is self-dual). By abuse of notation, we shall also refer to these morphisms by the notation $\mathscr{P}$. Now, first of all. since $\nabla_{\mathscr{E}}$ stabilizes the filtration $\mathscr{T} \subseteq \mathscr{E}$, by functoriality, $\mathscr{P}$ also respects this filtration. Secondly, since $\mathscr{T}$ and $\mathscr{O}_{X}$ clearly have $p$-curvature zero, $\mathscr{P}$ not only respects the filtration, but is nilpotent, i.e., $\mathscr{P}: \mathscr{T} \rightarrow$ Ad $(\mathscr{E})$ maps into the subbundle $\mathscr{T} \subseteq$ Ad $(\mathscr{E})$ (given by endomorphisms of $\mathscr{E}$ obtained by projecting $\mathscr{E} \rightarrow \mathscr{O}_{X}$. mapping $\mathscr{O}_{X}$ to $\mathscr{T}$, and then injecting $\left.\mathscr{T} \hookrightarrow \mathscr{E}\right)$. Thus, $\mathscr{P}$ basically amounts to a morphism from $\mathscr{T}$ to $\mathscr{T}$, i.e., a section of $f_{*} \mathscr{O}_{X}=$ $\mathfrak{O}_{S}$.

Proposition 1.4. Assume that $p$ is odd. If $\left(\mathscr{E}, \nabla_{\mathscr{E}}\right)$ is an FL-bundle on $X^{\mathrm{log}}$, then $\mathscr{P}: \mathscr{T} \rightarrow \operatorname{Ad}(\mathscr{E})$ is given by multiplication $\mathscr{T} \rightarrow \mathscr{T}$ by -1 , followed by the 
inclusion $\mathscr{T} \hookrightarrow \operatorname{Ad}(\mathscr{E})$.

Proof. Since the universal example of an FL-bundle on an $r$-pointed stable curve of genus $g$ is given by the torsor $\mathscr{A}$ over $\bar{M}_{g, r}$. which is smooth, it suffices to check the assertion after restriction to a closed point of this universal $\mathscr{A}$. Thus, we can assume that $S=\operatorname{Spec}(k)$, where $k$ is a finite field. Then $S$ has a canonical lifting to a flat scheme over $\mathbb{Z} / p^{2} \mathbf{Z}$. namely, $\widetilde{S}=\operatorname{Spec}\left(W(k) / p^{2} W(k)\right)$ (where $W(k)$ is the ring of Witt vectors with coefficients in $k$ ), and $\widetilde{S}$ has a natural Frobenius lifting $\Phi_{\tilde{S}}$. Thus, for any smooth scheme $\widetilde{U} \rightarrow \widetilde{S}$, we may speak of a Frobenius lifting $\left(\bmod p^{2}\right)$ on $\widetilde{U}$, that is, an $\widetilde{S}$-linear morphism $\Phi: \widetilde{U}$ $\rightarrow \widetilde{U}^{F}$ whose reduction modulo $p$ is the relative Frobenius $U \rightarrow U^{F}$ of $U^{\text {def }}=\widetilde{U} \otimes$ $\mathbb{Z} / p \mathbb{Z}$.

Now let us take $U$ to be an affine open subset $L \subseteq X$, at which $f$ is smooth, and which contains no marked points. Let $\widetilde{U} \rightarrow \widetilde{S}$ be a smooth lifting of $U$, and let $t$ be a local coordinate on $U$. By the interpretation of FL-bundles in terms of obstructions to Frobenius liftings, we may compute $\mathscr{E}$ by using as follows: Over $U,\left.\left.\mathscr{E}\right|_{U} \cong \mathscr{T}\right|_{U} \oplus \mathscr{O}_{U}$. Let us write sections of $\mathscr{E}$ relative to the decomposition $\left.\mathscr{T}\right|_{U} \oplus \mathscr{O}_{U}$ and the basis given by $\left(\Phi_{X^{\log } / S^{\log }}^{-1}\left(\frac{d}{d t}\right)^{F}, 0\right) ;(0,1)$; and let us denote by $\nabla$ the connection $\nabla_{\mathscr{s}}$ applied in the direction $\frac{d}{d t}$. Then $\nabla$ is given by adding to the direct sum connection the matrix

$$
\left(\begin{array}{cc}
0 & \frac{1}{p} \Phi^{\prime} \\
0 & 0
\end{array}\right)
$$

where the map $\Phi^{\prime}$ is the derivative (with respect to $t$ ) of some local Frobenius lifting $\Phi$ on $\widetilde{U}$. Since $t^{F} \mapsto(1+t)^{p}-1$ is a Frobenius lifting, $\Phi\left(t^{F}\right)$ must be of the form $(1+t)^{p}-1+p \cdot f(t)$. for some function $f(t)$ on $U$. Therefore, $\frac{1}{p} \Phi^{\prime}$ is of the form $(1+t)^{p-1}+f^{\prime}(t)$. This gives $\nabla(1,0)=0: \nabla(0,1)=\left((1+t)^{p-1}+f^{\prime}, 0\right)$. Therefore, $(\nabla)^{p}(1,0)=0$ and $\nabla^{p}(0,1)=(p-1) !(1,0)$ (since $\left(\frac{d}{d t}\right)^{p} f=0$ ).

Finally, it follows easily from using the fact that $\mathbf{F}_{p}^{\times}$is a cyclic group that $(p-$ $1) !=-1\left(\right.$ in $\mathbb{F}_{p}$ ). This completes the proof.

Corollary 1.5. Assume that $p$ is odd. Let $\left(\mathscr{E}, \nabla_{\mathscr{E}}\right)$ be a rank two vector bundle with logarithmic connection on $X^{\log }$ (over $S^{\mathrm{log}}$ ) defined by a section $\eta$ of $\mathbb{R}^{1} f_{\mathrm{DR}, *}(\mathscr{T})$. Then $\left(\mathscr{E}, \nabla_{\mathscr{E}}\right)$ is an FL-bundle if and only if its p-curvature is nonzero at some point of every fiber of $f: X \rightarrow S$. 
Proof. The "only if" part follows from Proposition 1.4. On the other hand, suppose that the image of $\eta$ under the map $\mathbb{R}^{1} f_{\mathrm{DR}, *}(\mathscr{T}) \rightarrow \mathscr{O}_{S}$ of Proposition 1.1 vanishes at a point. By restricting to that point, we may assume that $S$ is the spectrum of a field, and that the image of $\eta$ in $\mathscr{O}_{S}$ vanishes identically. But then, it follows from the exact sequence of Proposition 1.1 that $\left(\mathscr{E}, \nabla_{\mathscr{E}}\right)$ is the pull-back of a bundle under Frobenius. Then its $p$-curvature must vanish identically, which contradicts our assumption.

Corollary 1.6. Assume that $p$ is odd and that $S$ is reduced. Let $\left(\mathscr{E}, \nabla_{\mathscr{E}}\right)$ be an FL-bundle. Let $U \subseteq X$ be an open subset; $\left(\mathscr{L}, \nabla_{\mathscr{L}}\right)$ be a line bundle with logar. ithmic connection on $U^{\mathrm{rog}}$; and $\iota:\left.\mathscr{L} \hookrightarrow \mathscr{E}\right|_{U}$ be a horizontal morphism of $\mathscr{O}_{U}$-modules with logarithmic connections. Then c factors through the injection $\left.\left.\mathscr{T}\right|_{U} \hookrightarrow \mathscr{E}\right|_{U}$ in the definition of $\mathscr{E}$ as an FL-bundle.

Proof. By shrinking $U$, we may assume that $f: X \rightarrow S$ is smooth on $U$, and that $U$ stays away from the marked points. We may also assume that the composite of $c$ with the projection $\left.\mathscr{E}\right|_{U} \rightarrow \mathscr{O}_{U}$ is an isomorphism. Thus, we obtain a horizontal isomorphism of line bundles $\mathscr{L} \rightarrow \mathscr{O}_{U}$. But this implies that $\left(\mathscr{L}, \nabla_{\mathscr{L}}\right)$ has $p$-curvature zero. Thus, we get a horizontal isomorphism $\left.\left.\mathscr{L} \oplus \mathscr{T}\right|_{U} \rightarrow \mathscr{E}\right|_{U^{\prime}}$. Since the left-hand side has $p$-curvature zero, the same is true of the right-hand side. But this contradicts Proposition 1.4.

Remark. It is not difficult to construct counterexamples to Corollary 1.6 if one does not assume that $S$ is reduced.

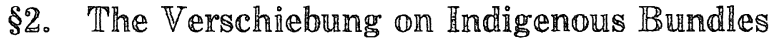

In this Section, we define a "Verschiebung" morphism on the space $\bar{\Phi}_{g . r}$ of Schwarz structures that takes values in the space of square differentials (twisted by Frobenius). We then prove various basic properties of this morphism, such as computing its derivative. This computation reveals that the derivative looks rather like the Verschiebung morphism for the Jacobian of the curve, thus justifying the terminology. On the other hand, as we shall see in $\S 3$, unlike the Verschiebung of the Jacobian which only pertains to $H^{1}$ of the curve, the Verschiebung on indigenous bundles pertains to a nonabelian invariant of the curve, namely, the nilpotent indigenous bundles on the curve. It turns out that the study of nilpotent indigenous bundles, and thus of the Verschiebung on indigenous bundles are central to understanding uniformization theory in the $p$-adic context. In this Section, $\bar{M}_{g, r}$ (respectively, $\overline{\mathscr{X}}_{g, r}$ ) will denote the moduli stack of $r$-pointed curves of genus $g$ (respectively, equipped 
with a Schwarz structure) over $\mathbf{F}_{p}$. We assume throughout this Section that $p$ is odd.

\section{The Definition of the Verschiebung}

Let $S^{\log }$ be a fine noetherian log scheme over $\mathbb{F}_{p}$. Let $f^{\text {log }}: X^{\log } \rightarrow S^{\log }$ be an $r$-pointed stable curve of genus $g$. Let $D \subseteq X$ be the divisor of marked points. Let $\left(\mathscr{E}, \nabla_{\mathscr{8}}\right)$ be an indigenous bundle on $X^{\mathrm{log}}$ (see $\S 2$ of Chapter I for more on such bundles). We remark here that throughout this paper, when we do varions manipulations with indigenous bundles, it will be simpler to work with vector bundles, rather than $\mathbb{P}^{1}$-bundles. Of course, indigenous veclor bundles only exist under certain conditions (cf. Proposition 2.6 of Chapter I). but this will not pose any problem, since we can always either Zariski localize on the curve, or pass to some sort of covering of the curve, and then descend for the final result. Thus, in the future, for the rest of the paper, we shall act as if this problem does not exist, and always work with indigenous vector bundles, when it is technically more comfortable to do so.

We maintain the notation of $\S 1$ for the various Frobenius morphisms and

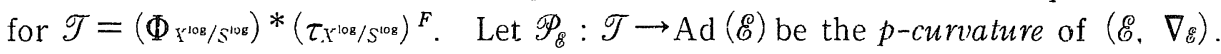
Consider the composite of $\mathscr{P}_{\mathscr{b}}$ with its dual $\mathscr{P}_{\mathscr{g}}^{\vee}$. This composite is a horizontal morphism $\mathscr{T} \rightarrow \mathscr{T}^{\vee}$, hence defines a section of $\left(f_{*}\left(\omega_{X / S}^{\log }\right)^{\otimes 2}\right)^{F}$. Let $\mathscr{V}_{\&}$ be $-\frac{1}{2}$ times this section. Another way to put the definition of $\mathscr{V}_{\mathscr{B}}$ is as follows: We consider the square $\left(\mathscr{P}_{\mathscr{E}}\right)^{2}:(\mathscr{T})^{\otimes 2} \rightarrow$ End $(\mathscr{E})$ of $\mathscr{P}_{\mathscr{g}}$, take the trace, and multiply by $-\frac{1}{2}$. Yet another way to put the definition of $\mathscr{V}_{\&}$ is that it is the determinant of $\mathscr{P}_{\mathscr{g}}\left(\right.$ regarded as a map $\left.(\mathscr{T})^{\otimes 2} \rightarrow \mathscr{O}_{X}\right)$.

Proposition 2.1. Assume that $S$ is reduced. Then $\mathscr{V}_{8}^{\prime}$ is zero if and only if the image of $\mathscr{P}_{\mathscr{E}}$ consists of nilpotent endonnorphisms of $\mathscr{E}$.

Proof. Immediate from the definitions.

Thus, we may think of $\mathscr{V}_{\mathscr{E}}$ as a measure of how nilpotent $\mathscr{P}_{\mathscr{B}}$ is. Note, in particu-

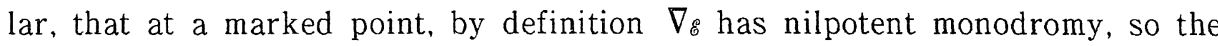
$p$-curvature is already nilpotent there. Thus, $\mathscr{V}_{\mathscr{E}}$ has zeroes at all the marked points. By abuse of notation, we shall denote by $\mathscr{V}_{\&}$ the resulting section of $\left(f_{*}\right.$ $\left.\left(\omega_{X / s}^{\log }\right)^{\otimes 2}(-D)\right)^{F}$.

Definition 2.2. We shall refer to the section $\mathscr{V}_{\mathscr{B}}$ of $\left(f_{*}\left(\omega_{X / S}^{\log }\right)^{\otimes 2}(-D)\right)^{F}$ as the Verschiebung of the indigenous bundle $\left(\mathscr{E}, \nabla_{\mathscr{E}}\right)$. 
Thus, in the universal case, when $f^{\log :} \mathscr{C}^{\log } \rightarrow \overline{\mathcal{M}}_{g, r}^{\text {og }}$ is the universal $r$-pointed stable curve of genus $g$. we obtain a morphism of $\bar{M}_{g, r}$-schemes:

$$
\bar{V}_{g, r}: \overline{\mathscr{Q}}_{g, r} \rightarrow \overline{\mathscr{Q}}_{g, r}
$$

where $\overline{\mathscr{Q}}_{g, r}$ is the geometric vector bundle corresponding to (i.e., Spec of the symmetric algebra of the dual of $)\left(f_{*}\left(\omega_{X / S}^{\log }\right)^{\otimes 2}(-D)\right)^{F}$. Note that both $\overline{\mathscr{\Phi}}_{g, r}$ and $\overline{\mathscr{Q}}_{g, r}$ are of dimension $3 g-3+r$ over $\bar{M}_{g, r}$. The rest of this Chapter will be devoted to studying this Verschiebung morphism $\bar{V}_{g, r}$.

Let us begin with some observations concerning the degree of $\bar{V}_{g, r}$ as a polynomial map. Let $\nabla_{\mathscr{E}}^{\prime}=\nabla_{\mathscr{f}}+\theta$ be a logarithmic connection on $\mathscr{E}$ that makes it an indigenous bundle; here we may assume that $\theta$ is an $\operatorname{Ad}(\mathscr{E})$-valued differential which defines a square-nilpotent endomorphism of $\mathscr{E}$ and which corresponds to a square differential $\theta^{\text {SD }}$. Thus, $\theta^{2}=0$. If we then compute the $p$-curvature of $\left(\mathscr{E}, \nabla_{\mathscr{g}}^{\prime}\right)$ by, say, working locally on $U \subseteq X$ where there is a local coordinate $x$, and letting $\nabla$ (respectively, $\nabla^{\prime} ; \theta_{x}$ ) denote $\nabla_{\mathscr{E}}$ (respectively, $\left.\nabla_{6}^{\prime} ; \theta\right)$ applied in the direction $\partial \stackrel{\text { def }}{=} \frac{d}{d x}$, we note that because $\theta_{x}^{2}=0$, all the terms that involve $\theta_{x}$ more than $\frac{1}{2}(p+1)$ times must vanish. Moreover, there is only one term that involves $\theta_{x}$ exactly $\frac{1}{2}(p+1)$ times, namely:

$$
\theta_{x} \nabla \theta_{x} \nabla \cdots \nabla \theta_{x}
$$

that is, alternating $\theta_{x}$ 's and $\nabla^{\prime}$ 's, with a total of $\frac{1}{2}(p+1)$ copies of $\theta_{x}$ and $\frac{1}{2}(p-$ 1 ) copies of $\nabla$. For future reference let us call this term $\xi$. Note that since $\theta_{x}^{2}=0$. any string $\theta_{x} \nabla \theta_{x}$ can be rewritten $\theta_{x}\left[\nabla, \theta_{x}\right]$ (where the brackets denote the commutator), and that this commutator $\left[\nabla, \theta_{x}\right]$ is a linear operator (that is, linear over $\mathscr{O}_{X}$ ). Moreover, this linear operator $\left[\nabla, \theta_{x}\right]$ preserves the Hodge filtration of $\mathscr{E}$. Thus, $\xi$ may be rewritten as $\theta_{x}$ times $\left[\nabla, \theta_{x}\right]$ to some power. Since $\left[\nabla, \theta_{x}\right]$ preserves the Hodge filtration, it thus follows that $\xi$ is a linear operator on $\mathscr{E}$ which is nilpotent with respect to the Hodge filtration. In particular, $\xi^{2}=0$. At any rate. we may at least conclude that in the expression for the trace of the square of the $p$-curvature of $\nabla_{g}^{\prime}, \theta$ occurs no more than $p$ times in each term. We thus obtain the following result:

(*) Relative to the affine structures of $\overline{\mathscr{Q}}_{g, r}$ and $\overline{\mathscr{Q}}_{g, r}$, the pull-backs of the affine variables on $\overline{\mathscr{Q}}_{g, r}$ via the morphism $\bar{V}_{g, r}$ are polynomials in the affine variables of $\overline{\mathscr{\Phi}}_{g, r}$ of degree $\leq p$. 
In fact, we would like to conclude a stronger result, namely that the degree is exactly $p$. In order to do this, we need to enlist the aid of Jacobson's formula (see, e.g.. [Jac].pp.186-187): This formula states that if $a$ and $b$ are elements of an associative ring $R$ of characteristic $p$, then

$$
(a+b)^{p}=a^{p}+b^{p}+\sum_{i=1}^{p-1} s_{l}(a, b)
$$

where the $s_{t}(a, b)$ are given by the formula:

$$
(\operatorname{ad}(t a+b))^{p-1}(a)=\sum_{i=1}^{p-1} i s_{l}(a, b) t^{i-1}
$$

computed in the ring $R[t]$, where $t$ is an indeterminate. In our case. we let $b=$ $\nabla$ and $a=\theta_{x}$, and we wish to compute the $s,(a, b)$, where $j=\frac{1}{2}(p-1)$. Let $\eta$ be the coefficient multiplying $t^{j-1}$ in the expression $(\operatorname{ad}(t a+b))^{p-1}(a)$. Let $\alpha=$ ad $(a) ; \beta=\operatorname{ad}(b)$. Then the terms in $\eta$ look like $\frac{1}{2}(p-3)$ copies of $\alpha$ and $\frac{1}{2}(p$ +1 ) copies of $\beta$ applied to $a$ in some order. Now ultimately, in order to compute the Verschiebung, we are interested in computing $\operatorname{tr}(\eta \cdot \xi)$. Let $\tau$ be one of the terms that make up $\eta$. Now we separate the analysis of $\tau$ into two cases: the case where $\tau$ begins with an $\alpha$, and the case where $\tau$ begins with a $\beta$.

Suppose that $\tau$ begins with an $\alpha$. Thus, $\tau$ looks like

$$
\alpha \gamma_{1} \cdots \gamma_{p-2} a
$$

where each $\gamma_{1}$ is either $\alpha$ or $\beta$. Now let us note that when one applies $\alpha=$ ad $\left(\theta_{x}\right)$ or $\beta=\operatorname{ad}(\nabla)$ to a linear operator on $\mathscr{E}$, one gets back a linear operator. Moreover, $\alpha$ applied to any linear operator on $\mathscr{E}$ yields a linear operator that preserves the Hodge filtration on $\mathscr{E}$. Thus, we conclude that $\tau$ is a linear operator on $\mathscr{E}$ that preserves the Hodge filtration. Since $\xi$ is nilpotent with respect to the Hodge filtration, it thus follows that $\operatorname{tr}(\tau \cdot \xi)=0$.

Now suppose that $\tau$ begins with a $\beta$ :

$$
\beta \gamma_{1} \cdots \gamma_{p-2} a
$$

Let us denote by $\sigma$ the linear operator on $\mathscr{E}$ given by $\gamma_{1} \cdots \gamma_{p-2} a$ (i.e., we leave off the initial $\beta$ ). We would like to show that $\sigma$ preserves the Hodge filtration on $\mathscr{E}$. To see this. first note that among the $\gamma_{i}$ 's. the number of $\beta$ 's is exactly one greater than the number of $\alpha$ 's. Also, note that (by Griffiths transversality) relative to the Hodge filtration on $\operatorname{Ad}(\mathscr{E})$, applying $\beta$ decreases the filtration index $l$ (in $F^{l}(\operatorname{Ad}(\mathscr{E}))$ ) by at most one, while applying $\alpha$ always 
increases the filtration index $l$ by one. Thus, since $a=\theta_{x} \in F^{1}(\operatorname{Ad}(\mathscr{E}))$, it follows that $\sigma \in F^{0}(\operatorname{Ad}(\mathscr{E}))$, i.e., it fixes the Hodge filtration on $\mathscr{E}$, and so $\sigma \cdot \xi$ is nilpotent with respect to the Hodge filtration. In particular, $\operatorname{tr}(\sigma \cdot \xi)=0$. Since the trace map is horizontal, we thus obtain that $\operatorname{tr}([\nabla, \sigma \circ \xi])=0$. Therefore, when we multiply $\tau=\beta(\sigma)$ by $\xi$ and take the trace, we get

$$
\begin{aligned}
\operatorname{tr}(\tau \cdot \xi) & =\operatorname{tr}([\nabla, \sigma \cdot \xi])-\operatorname{tr}(\sigma \cdot[\nabla, \xi]) \\
& =-\operatorname{tr}(\sigma \cdot[\nabla, \xi])
\end{aligned}
$$

In other words, $\operatorname{tr}(\tau \cdot \xi)$ depends only on the images of $\sigma$ and $[\nabla, \xi]$ in $F^{0} / F^{1}$ $(\operatorname{Ad}(\mathscr{E}))$.

To compute the image of $\sigma$ in $F^{0} / F^{1}(\operatorname{Ad}(\mathscr{E}))$, we must analyze $\sigma$ in greater detail. Now we saw that when we compute $\sigma$ by applying $\alpha$ 's and $\beta$ 's to $a, \beta$ decreases the filtration index by at most one. The only time it fails to decrease the filtration index by one is when it is applied to a linear operator which already has a nontrivial image in $F^{-1} / F^{0}(\operatorname{Ad}(\mathscr{E}))$. If this should occur even once, then the net change in the filtration index as a result of applying all the $\gamma_{i}$ 's (in the computation of $\sigma$ ) to $a$ is $\leq 0$. Thus, if this occurs even once, $\sigma \in F^{1}$ $(\operatorname{Ad}(\mathscr{E}))$, so $\operatorname{tr}(\sigma \cdot[\nabla, \xi])=0$. On the other hand, if, during the calculation of $\sigma$, we apply $\alpha$ to a linear operator in $F^{1}(\operatorname{Ad}(\mathscr{E}))$, we get zero. Let us call the case where neither of these two phenomena ever occurs the nondegenerate case. Thus, only the nondegenerate terms $\tau$ will make a nonzero contribution.

Let us suppose that $\tau$ is nondegenerate. Then in order to compute the image of $\sigma$ in $F^{0} / F^{1}(\operatorname{Ad}(\mathscr{E}))$. it suffices to merely keep track of the leading term (relative to the Hodge filtration on $\operatorname{Ad}(\mathscr{E})$ ) as we apply the various $\gamma_{i}$ 's. Now let us note that it follows from the fact that the Kodaira-Spencer morphism for the Hodge filtration on $\mathscr{E}$ is the identity that if $l \geq 1$, then applying $\beta$ to a linear operator $L$ in $F^{l}(\mathrm{Ad}(\mathscr{E}))$ yields a linear operator in $F^{l-1}(\mathrm{Ad}(\mathscr{E}))$ whose image in $F^{l-1} / F^{l}(\operatorname{Ad}(\mathscr{E}))$ is the image of $L$ in $F^{l} / F^{l+1}(\operatorname{Ad}(\mathscr{E}))$ times $\partial$. On the other hand, if $l \leq 1$, then applying $\alpha$ to a linear operator $L$ in $F^{l} \operatorname{Ad}(\mathscr{E})$ yields a linear operator in $F^{l+1}(\operatorname{Ad}(\mathscr{E}))$ whose image in $F^{l+1} / F^{l+2}(\operatorname{Ad}(\mathscr{E}))$ is the image of $L$ in $F^{l} / F^{l+1}(\operatorname{Ad}(\mathscr{E}))$ times $\theta^{\mathrm{SD}} \cdot \partial$. Thus, if $\tau$ is nondegenerate, then its contribution $-\operatorname{tr}(\sigma \cdot[\nabla, \xi])$ is given by $\left(\theta^{\mathrm{SD}} \cdot \partial\right)^{p} \cdot(\partial)^{p}$.

It remains to compute the number of nondegenerate terms $\tau$. Let us call this number $N_{p}$, and regard it as an element of $\mathbb{F}_{p}$. We are interested in whether or not $N_{p} \in \mathbb{F}_{p}$ is zero. Although one can presumably compute $N_{p}$ explicitly using some sort of combinatorial argument, we prefer to take the following approach. Note that $N_{p}$ does not depend on $g$ or $r$ or on the particular curve $f^{\log }: X^{\log } \rightarrow S^{\log }$, but only on $p$. Thus, it suffices to show that (for each 
odd prime $p$ ) $N_{p} \neq 0$ for one particular curve (with $g$ and $r$ arbitrary). We shall do this in $\S 3$ when we do various computations with elliptic curves. Thus, we obtain that $\mathscr{V}_{\mathscr{E}}$ (which is a section of $\left.\left(f_{*}\left(\omega_{X / S}^{\log }\right) \otimes 2(-D)\right){ }^{F}\right)$, when applied to $\left(\partial^{F}\right)^{2}$ yields the function $c \cdot\left(\theta^{\mathrm{SD}} \cdot \partial\right)^{p} \cdot(\partial)^{p}$, where $c \in \mathbf{F}_{p}^{\times}$, plus lower degree terms in $\theta^{\mathrm{SD}}$. Put another way, the degree $p$ component of $\overline{\mathscr{V}}_{g, r}$ is a morphism:

$$
\overline{\mathscr{V}}_{g, r}^{[p]}:\left\{\left(f_{*}\left(\omega_{X / S}^{\log }\right)^{\otimes 2}(-D)\right)^{\vee}\right\}^{F} \rightarrow \mathbf{S}^{p}\left(f_{*}\left(\omega_{X / S}^{\log }\right)^{\otimes 2}(-D)\right)^{\vee}
$$

which is equal to $c$ times the Frobenius morphism, i.e., the $p^{\text {th }}$-power map. We shall see later in our computations with elliptic curves that $c=-1$. Thus, we obtain the following important result:

Theorem 2.3. Relative to the affine structures of $\overline{\mathscr{ष}}_{g, r}$ and $\overline{\mathscr{Q}}_{g, r}$, the pull-backs of the affine variables on $\overline{\mathscr{Q}}_{g, r}$ via the morphism $\overline{\mathscr{V}}_{g, r}$ are polynomials in the affine variables of $\overline{\mathscr{X}}_{g, r}$ of degree exactly $p$, with the leading term $\overline{\mathcal{V}}_{g, r}^{[p]}(i . e .$, the degree $p$ component) given by -1 times the $p^{\text {th }}$-pouer map. In particular, $\bar{V}_{g, r}$ is a finite. flat morphism of degree $p^{3 g-3+r}$.

Proof. It remains to verify the last assertion (that $\bar{V}_{g, r}$ is finite and flat of the right degree). Let $U=\operatorname{Spec}(A) \rightarrow \bar{M}_{g, r}$ be étale. Then over $U$, we may choose affine coordinates $X_{l}$ and $Y$, of $\overline{\mathscr{Q}}_{g, r}$ and $\overline{\mathscr{Q}}_{g, r}$ so that $\overline{\mathcal{V}}_{g, r}$ looks like (the map induced on Spec's by):

$$
B \stackrel{\text { def }}{=} A\left[Y_{1} \cdots, Y_{3 g-3+r}\right] \rightarrow C \stackrel{\text { def }}{=} A\left[X_{1}, \cdots, X_{3 g-3+r}\right]
$$

where $Y_{\imath} \mapsto f_{\imath}\left(X_{1}, \cdots, X_{3 g-3+r}\right)$, and $f_{\imath}$ is of the form " $-X_{\imath}^{p}$ plus terms of lower degree." Then it is easy to see that, as a $B$-module. $C$ is generated by monomials of the form

$$
\prod_{i=1}^{3 g-3+r} X_{\imath}^{e_{l}}
$$

where $0 \leq e_{e_{1}} \leq p-1$. In particular, $C$ is a finite $B$-module, so $\bar{V}_{g, r}$ is finite. Since $\bar{V}_{g, r}$ is a finite morphism between regular algebraic stacks of the same dimension, it follows from commutative algebra that $\bar{V}_{g, r}$ is flat. To compute the degree of $\bar{V}_{g, r}$, let $P=\operatorname{Proj}_{A}\left(C[T]\right.$ ) (with the grading such that $T$ and the $X_{i}$ 's have degree one). Then $\operatorname{Spec}(C) \subseteq P$ is an affine open subset. Let $S_{\imath} \subseteq P$ be the hypersurface which is the closure of the zero locus of $f_{i}$. Then the scheme-theoretic intersection $V$ of all the $S_{\imath}$ 's has degree $p^{3 g-3+r}$ over $\operatorname{Spec}(A)$. Also, the intersection of $V$ with $P-\operatorname{Spec}(C)=V_{+}(T)=\operatorname{Proj}(C)$ is just $V_{+}$ 
$\left(X_{1}^{p}, \cdots, X_{3 g-3+r}^{p}\right) \subseteq \operatorname{Proj}(C)$, which is the empty set. Thus, $V \subseteq \operatorname{Spec}(C)$, and so $\operatorname{deg}\left(\overline{\mathscr{V}}_{g, r}\right)=\operatorname{deg}(V \rightarrow \operatorname{Spec}(A))=p^{3 g-3+r}$.

Before continuing, we introduce some more terminology that will be of use in the future:

Definition 2.4. Let $\left(\mathscr{E}, \nabla_{\mathscr{E}}\right)$ be an indigenous bundle on $X^{\mathrm{log}}$. Then, we will say that $\left(\mathscr{E}, \nabla_{\mathscr{E}}\right)$ is nilpotent if $\mathscr{V}_{\delta}$ is zero. We will say that $\left(\mathscr{E}, \nabla_{\mathscr{E}}\right)$ is admissible if $\mathscr{P}_{\mathscr{E}}^{\vee}: \operatorname{Ad}(\mathscr{E}) \rightarrow \mathscr{T}^{\vee}$ is surjective.

Note that the terminology of a "nilpotent indigenous bundle" that we have introduced here is (by Proposition 2.1) consistent with that of [Katz]. Also, let us observe that the nilpotent bundles form a closed subscheme

$$
\overline{\mathcal{N}}_{g, r} \subseteq \overline{\mathscr{\otimes}}_{g, r}
$$

while the admissible bundles form an open subscheme

$$
\overline{\mathscr{\varnothing}}_{g, r}^{\mathrm{adm}} \subseteq \overline{\mathscr{\vartheta}}_{g, r}
$$

We shall see later in this Chapter that $\overline{\mathscr{X}}_{g, r}^{\text {adm }} \cap \overline{\mathcal{N}}_{g, r}$ is nonempty and that neither $\overline{\mathscr{S}}_{g, r}^{\mathrm{adm}}$ nor $\overline{\mathcal{N}}_{g, r}$ is contained in the other. Note, further, that Theorem $2.3 \mathrm{im}$ plies that the natural morphism

$$
\overline{\mathcal{N}}_{g, r} \rightarrow \bar{M}_{g, r}
$$

is finite and flat of degree $p^{3 g-3+r}$.

Finally, we observe that one thing which is interesting about nilpotent indigenous bundles is that (if $S$ is the spectrum of a perfect field. then) by a result of [Falt] such indigenous bundles arise as the crystalline Dieudonne modules of certain finite. flat group schemes on $X^{\log }$. This point of view will be pursued further in later chapters. This observation is the main reason for studying $\overline{\mathcal{N}}_{g, r}$ and $\overline{\mathcal{V}}_{g, r}$, which is the goal of the present Chapter.

\section{The $\mathbb{P}^{-C}$-Curvature of an Admissible Indigenous Bundle}

As a prélude to finding out more about $\bar{V}_{g, r}$, it is worth looking at various basic properties of the $p$-curvature of an admissible indigenous bundle.

Proposition 2.5. There is a bijective correspondence between nilpotent, admissible indigenous bundles (up to tensor product with a line bundle of order two) and $\mathrm{FL}$-bundles whose projectivizations are indigenous given as follows: If $\left(\mathscr{E}, \nabla_{\mathscr{E}}\right)$ 
is a nilpotent, admissible indigenous bundle on $X^{\mathrm{log}}$, let $\mathscr{P}_{\mathscr{E}}: \mathscr{T} \rightarrow$ Ad $(\mathscr{E})$ be its p-curvature. Then the kernel of $\mathscr{P}_{\mathscr{g}}^{\vee}: \operatorname{Ad}(\mathscr{E}) \rightarrow \mathscr{T}^{\vee}$ is an FL-bundle.

Moreover, if $\left(\mathscr{E}, \nabla_{\mathscr{E}}\right)$ is a nilpotent, admissible indigenous bundle on $X^{\mathrm{log}}$, there exists a unique rank one subbundle $M \subseteq \mathscr{E}$ that is annihilated by the endomorphisms in the image of $\mathscr{P}_{\mathscr{E}}$. This subbundle $\mathcal{M}$ is stabilized by $\nabla_{\mathscr{B}}$. The induced connection $\nabla \mu$ has p-curvature zero, and we have a horizontal isomorphism $\mathcal{M}^{\otimes 2} \cong \mathscr{T}$.

Finally, suppose that $S$ is reduced, and $\left(\mathscr{E}, \nabla_{\mathscr{E}}\right)$ is nilpotent, admissible, and indigenous. Let $U \subseteq X$ be an open set, and $\left(\mathscr{L}, \nabla_{\mathscr{L}}\right)$ a line bundle with logarithmic connection (with respect to $f^{\mathrm{lug}}: X^{\mathrm{log}} \rightarrow S^{\mathrm{log}}$ ) on $U$. Let $\iota:\left.\mathscr{L} \rightarrow \mathscr{E}\right|_{U}$ be a horizontal

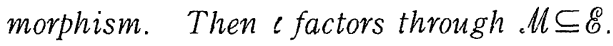

Proof. First of all, since $\left(\mathscr{E}, \nabla_{\mathscr{E}}\right)$ is admissible, $\mathscr{P}_{\mathscr{E}}^{\vee}$ is surjective, and thus its kernel is a rank two vector bundle $\mathscr{F}$ which is stabilized by $\nabla_{\mathscr{E}}$, hence gets a connection $\nabla_{\mathscr{F}}$. Since $\left(\mathscr{E}, \nabla_{\mathscr{E}}\right)$ is nilpotent, it follows that $\mathscr{T} \cong \operatorname{Im}\left(\mathscr{P}_{\mathscr{E}}\right) \subseteq \mathscr{F}$, and that this inclusion $\mathscr{T} \hookrightarrow \mathscr{F}$ is locally split. Also, this inclusion $\mathscr{T} \hookrightarrow \mathscr{F}$ is necessarily horizontal, and we also have a horizontal isomorphism $\mathscr{F} / \mathscr{T} \cong \mathscr{O}_{X}$. Thus, in order to show that $\left(\mathscr{F}, \nabla_{\mathscr{F}}\right)$ is an FL-bundle. it suffices to show that the $p$-curvature of $\left(\mathscr{F}, \nabla_{\mathscr{F}}\right)$ is nonzero generically on every fiber of $f: X \rightarrow S$ (by Corollary 1.5). Thus, we may assume that $S$ is the spectrum of a field. Now on some nonempty open set $L^{T} \subseteq X$, there is line bundle with connection $\left(\mathscr{L}, \nabla_{\mathscr{L}}\right)$ and a horizontal surjection $\mu: \mathscr{E} \rightarrow \mathscr{L}$ such that $\operatorname{Im}\left(\left.\mathscr{P}_{\mathscr{E}}\right|_{U}\right)$ is given by endomorphisms that vanish on $\operatorname{Ker}(\mu)$ and whose image is in $\operatorname{Ker}(\mu)$. Then sorting through what we have done, we see that we have a horizontal isomorphism $\left.\mathscr{F}\right|_{U}$ $\left.\cong \mathscr{E}\right|_{U} \otimes \mathscr{L}^{-1}$. Since $\mathscr{L}$ has $p$-curvature zero. the fact that $\left.\mathscr{E}\right|_{U}$ has nonzero $p$-curvature implies that the same is true of $\left.\mathscr{F}\right|_{U}$. This completes the proof that $\left(\mathscr{F}_{\mathscr{F}}, \nabla_{\mathscr{F}}\right)$ is an FL-bundle.

On the other hand, if we are given an FL-bundle $\left(\mathscr{F}, \nabla_{\mathscr{F}}\right)$ whose projectivization $\left(P, \nabla_{P}\right)$ is indigenous, let $\left(\mathscr{E}, \nabla_{\mathscr{E}}\right)$ be an indigenous vector bundle whose projectivization is $\left(P, \nabla_{P}\right)$. Then there exists a line bundle with connection $(\mathscr{L}, \nabla \mathscr{Q})$ (relative to $f^{\mathrm{log}}: X^{\log } \rightarrow S^{\log }$ ) such that we have a horizontal isomorphism $\mathscr{F} \otimes_{\mathbb{O} X} \mathscr{L} \cong \mathscr{E}$. Taking determinants, we thus get a horizontal isomorphism $\mathscr{L}^{\otimes 2} \cong \mathscr{T}^{\vee}$. so the $p$-curvature of $\mathscr{L}$ must be zero. Thus, under the natural identification of $\operatorname{Ad}(\mathscr{F})$ with $\operatorname{Ad}(\mathscr{E})$, we see that the $p$-curvatures of $(\mathscr{E}$, $\left.\nabla_{\mathscr{E}}\right)$ and $\left(\mathscr{F}, \nabla_{\mathscr{F}}\right)$ coincide. Thus, by our computation in Proposition $1.4,(\mathscr{E}$, $\left.\nabla_{\mathscr{E}}\right)$ is admissible and nilpotent. Also, it is easy to see that these two procedures are inverse to one another, thus proving the bijective correspondence. We take $M \subseteq \mathscr{E}$ to be $\mathscr{T} \otimes \mathscr{L} \subseteq \mathscr{F} \otimes \mathscr{L} \cong \mathscr{E}$. The remaining assertions follow immediately from what we have done so far, plus Proposition 1.6. 
Proposition 2.6. Let $\left(\mathscr{E}, \nabla_{\mathscr{E}}\right)$ be an indigenous bundle on $X^{\mathrm{log}}$. Let $\mathscr{P}_{\mathscr{E}}: \mathscr{T}$ $\rightarrow \operatorname{Ad}(\mathscr{E})$ be its p-curvature. Then :

(1) We shall call the composite $H_{\mathscr{E}}: \mathscr{T} \rightarrow \tau_{X^{108} / S^{108}}$ of $\mathscr{P}_{\mathscr{B}}$ with the projection $\mathscr{E} \rightarrow \tau_{X^{108} / S^{\text {log }}}$ arising from the Hodge filtration on $\mathscr{E}$ the square Hasse invariant of $\left(\mathscr{E}, \nabla_{\mathscr{E}}\right)$. If $\left(\mathscr{E}, \nabla_{\mathscr{E}}\right)$ is admissible, then $\mathrm{Hg}_{\mathrm{g}}$ is nonzero.

(2) Suppose that $\left(\mathscr{E}, \nabla_{\mathscr{E}}\right)$ is admissible. Then the zero locus $V\left(H_{\mathscr{g}}\right)$ $\subseteq X$ is a divisor $D_{g}$ which is finite, flat, and of degree $(p-1)(2 g$ $-2+r$ ) over $S$. We shall call it the double supersingular divisor of $\left(\mathscr{E}, \nabla_{\mathscr{E}}\right)$.

(3) Suppose that the indigenous bundle $\left(\mathscr{E}, \nabla_{\mathscr{E}}\right)$ is admissible and nilpotent. Then there exists a line bundle $\mathscr{H}$ on $X$ whose square $\mathscr{H}^{\otimes 2}$ is isomorphic to $\mathscr{T}^{\vee} \otimes \tau_{X^{108} / S^{108} \text {, together with a section } \chi \text { of }}$ H over $X$ whose square is $H_{g}$. We shall call $\chi$ the Hasse invariant of $\left(\mathscr{E}, \nabla_{\mathscr{G}}\right)$. In particular, there exists a divisor $E_{\mathscr{E}} \subseteq X$ such that $D_{\mathscr{E}}=2 E_{\mathscr{E}}$. We shall call E\& the supersingular divisor of $\left(\mathscr{E}, \nabla_{\mathscr{E}}\right)$.

(4) Suppose that $S$ is reduced. Then any two nilpotent, admissible indigenous bundles with the same supersingular locus are isomor. phic.

Proof. For (1), it suffices to prove the statement after we restrict to a fiber of $f: X \rightarrow S$; thus, we may assume that $S$ is the spectrum of a field. If $H_{\mathscr{E}}$ were zero, then that would mean that $\operatorname{Im}\left(\mathscr{P}_{\mathscr{E}}\right)$ lands in $F^{0}(\operatorname{Ad}(\mathscr{E}))$. Now $F^{0}(\operatorname{Ad}(\mathscr{E}))$ surjects onto $\mathscr{O}_{X}$. If the image of $\mathscr{P}_{\mathscr{g}}$ in $\mathscr{O}_{X}$ is nontrivial, we get a contradication as follows: On the one hand, $\operatorname{Im}\left(\mathscr{P}_{\mathscr{\xi}}\right)$ is stabilized by $\nabla_{\varepsilon}$. On the other hand, the fact that the Kodaira-Spencer morphism of the Hodge filtration is an isomorphism means that $\nabla_{\mathscr{E}}$ applied to $\operatorname{Im}\left(\mathscr{P}_{\mathscr{g}}\right)$ will not be in $F^{0}(\operatorname{Ad}(\mathscr{E}))$. If the image of $\mathscr{P}_{\mathscr{B}}$ in $\mathscr{O}_{X}$ is trivial, then it must lie in $F^{1}(\operatorname{Ad}(\mathscr{E})) \cong \omega_{X / S}^{\log }$. Then, by using the fact that the Kodaira-Spencer morphism is an isomorphism, we again get a contradiction. Assertion (2) follows immediately from (1).

Now suppose that $\left(\mathscr{E}, \nabla_{\mathscr{E}}\right)$ is nilpotent. admissible, and indigenous. Let $\mathcal{M}$ $\subseteq \mathscr{E}$ be the rank one subbundle of Proposition 2.5. Let $\mathscr{E} \rightarrow \mathcal{N}$ be the surjection arising from the Hodge filtration. Then composing the injection $\mathcal{M} \hookrightarrow \mathscr{E}$ with this surjection, we get a morphism $\chi: \mathcal{M} \hookrightarrow \mathcal{N}$, whose square (under the identifications $\mathcal{M}^{\otimes 2} \cong \mathscr{T} ; \mathcal{N}^{\otimes 2} \cong \tau_{\left.X^{108} / S^{108}\right)}$ is equal to the Hasse invariant. Thus, if we let $E_{\mathscr{E}}$ be the zero locus of $\chi$, we have $D_{\mathscr{E}}=2 E_{\mathbb{E}}$. This proves (3).

To prove (4), we assume that $S$ is the spectrum of a field, and that we 
have two connections $\nabla_{\mathscr{E}}$ and $\nabla_{\mathscr{E}}^{\prime}$ on $\mathscr{E}$, both of which make $\mathscr{E}$ a nilpotent, admissible indigenous bundle, and such that the respective supersingular divisors $E_{\mathscr{g}}$ and $E_{\mathscr{E}}^{\prime}$ coincide. Let us also assume that $\nabla_{\mathscr{g}}^{\prime}=\nabla_{\mathscr{E}}+\theta$, where $\theta$ is a square differential. Let $\iota: \mathcal{M} \rightarrow \mathscr{E}$ and $\iota^{\prime}: \mathcal{M}^{\prime} \rightarrow \mathscr{E}$ be the respective inclusions, and $\chi: M \rightarrow \mathcal{N}$ and $\chi^{\prime}: \mathscr{M}^{\prime} \rightarrow \mathcal{N}$ the respective composites discussed in the preceding paragraph. We claim first of all that $\mathcal{M}$ and $\mathcal{M}^{\prime}$ are isomorphic. Indeed, this follows from the fact that $\mathcal{N} \otimes \mathcal{M}^{-1} \cong \mathscr{O}_{X}\left(E_{\mathscr{g}}\right)=\mathscr{O}_{X}\left(E_{\mathscr{E}}^{\prime}\right) \cong \mathcal{N} \otimes\left(\mathcal{M}^{\prime}\right)^{-1}$. Thus, we shall henceforth identify $\mathcal{M}$ and $\mathcal{M}^{\prime}$. Now $\chi$ and $\chi^{\prime}$ differ by multiplication by a section $\lambda$ of $\mathscr{O}_{s}$, that is, $\chi=\lambda \cdot \chi^{\prime}$. Let $i_{1}: \mathcal{M} \rightarrow \mathscr{E}$ be $\iota$, and let $i_{2}$ : $M \rightarrow \mathscr{E}$ be $i^{\prime}$ multiplied by $\lambda$. Then it follows that there exists a morphism $\alpha: \mathcal{M}$ $\rightarrow F^{1}(\mathscr{E})$ such that $i_{1}=i_{2}+\alpha$. Now let $s$ be a horizontal section of $\mathcal{M}$ (over some open set $U \subseteq X)$. Since the $p$-curvature is a horizontal morphism, $\nabla_{g}\left(i_{1}(s)\right)=0$ and $\nabla_{\mathscr{E}}^{\prime}\left(i_{2}(s)\right)=0$. Thus, we compute :

$$
\begin{aligned}
\nabla_{\mathscr{E}}^{\prime}\left(i_{2}(s)\right) & =\left(\nabla_{\mathscr{E}}+\theta\right)\left(i_{1}(s)+\alpha(s)\right) \\
& =\nabla_{\mathscr{E}}(\alpha(s))+\theta\left(i_{1}(s)\right)
\end{aligned}
$$

Suppose that $\alpha$ is nonzero. Then in the last line, the first term has a nonzero image under the surjection $\mathscr{E} \rightarrow \mathcal{N}$ (since the Kodaira-Spencer morphism is an isomorphism), while the second term lies in $F^{1}(\mathscr{E})$. Since the sum of these terms is zero, we thus obtain a contradiction. Thus, $\alpha$ must be zero. Then we obtain that $\theta\left(i_{1}(s)\right)=0$, so (if $\theta \neq 0$, then) $i_{1}$ maps into $F^{1}(\mathscr{E}) \subseteq \mathscr{E}$. But then $\chi=0$, so by $(2)$, we again obtain a contradiction. This completes the proof of (4).

Proposition 2.7. Suppose that $S$ is reduced. Let $\left(\mathscr{E}, \nabla_{8}\right)$ be admissible indigenous on $X^{\mathrm{log}}$. Let $\mathscr{P}_{\mathscr{E}}: \mathscr{T} \rightarrow \operatorname{Ad}(\mathscr{E})$ be the p-curvature. Let $U \subseteq X$ be open, and let $\left(\mathscr{L}, \nabla_{\mathscr{L}}\right.$ ) be a line bundle with logarithmic connection (relative to $f^{\mathrm{log}}$ ) on $L^{T}$ whose p-curvature is zero. Let $\iota: \mathscr{L} \rightarrow \mathrm{Ad}(\mathscr{E})$ be a horizontal morphism. Then $\iota$ factors through $\mathscr{T} \cong \operatorname{Im}\left(\mathscr{P}_{g}\right)$.

Proof. By shrinking $L$, we may assume that $\mathscr{L}$ and $\left.\mathscr{T}\right|_{U}$ have horizontal generating sections $s$ and $t$, respectively. We may assume that $s$ and $t$ generate a subbundle $\left.\mathscr{G} \subseteq \operatorname{Ad}(\mathscr{E})\right|_{U}$ of rank two. If we take their commutator in $\operatorname{Ad}(\mathscr{E})$, we see that $[s, t]$ must be in $\mathscr{G}$. Indeed, if this were not the case, then the $p$-curvature of $\mathrm{Ad}(\mathscr{E})$ would be zero. But the $p$-curvature of $\mathrm{Ad}(\mathscr{E})$ is given by $\operatorname{Ad}\left(\mathscr{P}_{\mathscr{E}}\right)$ which is nonzero everywhere since $\left(\mathscr{E}, \nabla_{\mathscr{E}}\right)$ is admissible. This proves the claim. Thus, $\mathscr{G}$ is a Lie subalgebra of $\operatorname{Ad}(\mathscr{E})$, stabilized by the connection on $\mathrm{Ad}(\mathscr{E})$ and whose $p$-curvature is zero. Being of rank two, it is necessarily solvable, hence contains a nilpotent subalgebra $\mathscr{K} \subseteq \mathscr{G}$ which is stabilized by the connection on $\operatorname{Ad}(\mathscr{E})$ and has $p$-curvature zero. Now $\mathscr{K}$ defines a horizontal filtration $\left.\mathcal{N} \subseteq \mathscr{E}\right|_{U}$ with respect to which it is nilpotent. Since 
$(\mathcal{N})^{\otimes 2} \cong \mathscr{K}$, it follows that $\mathcal{N}$ has $p$-curvature zero. Let $\delta:$ End $\left(\left.\mathscr{E}\right|_{U}\right) \rightarrow \operatorname{Ad}(\mathscr{E})$ $\left.\right|_{U}$ be the canonical projection given by quotienting out by the scalar endomorphisms. Then clearly the image of $\left.\mathcal{N} \otimes \mathscr{E}\right|_{U} \subseteq$ End $\left(\left.\mathscr{E}\right|_{U}\right)$ (i.e., the endomorphisms that anihilate $\left.\mathcal{N} \subseteq \mathscr{E}\right|_{U}$ ) under $\delta$ is equal to $\mathscr{G}$. Since $\mathscr{G}$ and $\mathcal{N}$ have $p$-curvature zero, it thus follows that $\left.\mathscr{E}\right|_{U}$ has $p$-curvature zero, which contradicts the fact that $\left(\mathscr{E}, \nabla_{\mathscr{E}}\right)$ is admissible.

Proposition 2.8. Suppose that $f^{\mathrm{jog}}$ is obtained by gluing together various $f_{i}^{\mathrm{log}}$, as in the last subsection of Chapter $I, \S 2$. Suppose that $\left(\mathscr{E}, \nabla_{\mathscr{E}}\right)$ is nilpotent, admissible, and indigenous on $X^{\mathrm{log}}$. Then it is automatically of restrictable type.

Proof. The subbundle $\mathcal{M} \subseteq \mathscr{E}$ (of Proposition 2.5) is stabilized by $\mathscr{E}$ and has $p$-curvature zero. Thus, if we restrict to an irreducible component $X_{1}^{\log }$, the monodromy at any marked point of $X_{1}^{\log }$ must be nilpotent with respect to the filtration defined by $M \subseteq \mathscr{E}$. This completes the proof.

As mentioned earlier, the reason that we are interested in nilpotent, admissible indigenous bundles is that they define $M \mathscr{F}^{\nabla}$-objects in the sense of [Falt]. Let us suppose that $S=\operatorname{Spec}(k)$, where $k$ is a perfect field, and that $f$ : $X \rightarrow S$ is smooth. Let $\widetilde{S}=\operatorname{Spec}(A)$, where $A=W(k) / p^{2} W(k)$, and $W(k)$ is the ring of Witt vectors with coefficients in $k$. Let us denote by $\Phi_{A}$ the canonical Frobenius morphism on $\widetilde{S}$. We suppose that $S$ and $\widetilde{S}$ are endowed with the trivial $\log$ structures, and call the resulting $\log$ schemes $S^{\log }$ and $\widetilde{S}^{\mathrm{log}}$, respectively. Let $\widetilde{f}^{\log }: \widetilde{X}^{\log } \rightarrow \widetilde{S}^{\text {log }}$ be a smooth $r$-pointed curve of genus $g$ that lifts $f^{\text {log }}$. Let $(\mathscr{E}$. $\left.\nabla_{\mathscr{E}}\right)$ be an indigenous bundle on $X^{\text {log }}$. Let $\widetilde{\mathscr{E}}=F^{1}(\mathscr{E}) \oplus\left(\mathscr{E} / F^{1}(\mathscr{E})\right)$. Let $\widetilde{U} \subseteq \widetilde{X}$ be an open subset, and let $\Phi^{\log }: \widetilde{U}^{\log } \longrightarrow \widetilde{U}^{\text {log }}$ be a lifting of Frobenius. If $\varepsilon$ is a section of $\mathscr{E}$. let $\nabla_{\tilde{\delta}}^{\Phi}(e)$ be the section of $\Phi^{*}\left(\mathscr{E} / F^{1}(\mathscr{E})\right) \otimes \omega_{L}{ }^{108} / S^{108}$ obtained by applying $\nabla_{\mathscr{E}}$ to $e$ (and regarding the result modulo $F^{1}(\mathscr{E}) \otimes \omega^{\left({ }^{108} / S^{108}\right)}$ to get a section

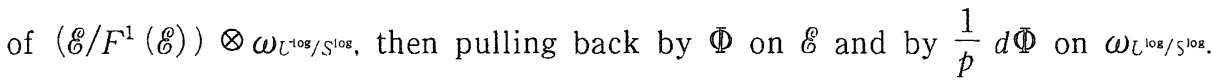
Then we can define a logarithmic connection $\nabla_{\Phi}$ on $\Phi^{*}\left(\widetilde{\mathscr{E}}^{F}\right)$ by letting $\nabla_{\Phi}\left(\Phi^{-1}\right.$ $\left.\left(0, e^{F}\right)\right)=0\left(\right.$ if $e$ is a section of $\left.\mathscr{E} / F^{1}(\mathscr{E})\right)$ and $\nabla_{\Phi}\left(\Phi^{-1}\left(e^{F}, 0\right)\right)=\left(0, \nabla_{\mathscr{E}}^{\Phi}(e)\right.$ ) (if $e$ is a section of $F^{1}(\mathscr{E})$ ). Then, it is easy to see (as in [Falt], §2) that for different $\Phi$, the pairs $\left(\left.\Phi^{*}\left(\widetilde{\mathscr{E}}^{F}\right)\right|_{\tilde{U}}, \nabla_{\Phi}\right)$ glue together to form a bundle with connection $\mathbb{F}^{*}\left(\mathscr{E}, \nabla_{\mathscr{E}}\right)$ on $X^{\mathrm{log}}$. Note that $\mathbb{F}^{*}\left(\mathscr{E}, \nabla_{\mathscr{E}}\right)$ depends on the choice of lifting $\widetilde{X}^{\log }$ $\rightarrow \widetilde{S}^{\text {log }}$.

Definition 2.9. We shall say that $\left(\mathscr{E}, \nabla_{\mathscr{E}}\right)$ forms an $\mathcal{M F}^{\nabla}$-object on $X^{\log }$ if 


$$
\left(\mathscr{E}, \nabla_{\mathscr{E}}\right) \otimes\left(\mathscr{L}, \nabla_{\mathscr{L}}\right) \cong \mathbb{F}^{*}\left(\mathscr{E}, \nabla_{\mathscr{E}}\right)
$$

for some choice of lifting $\widetilde{X}^{\text {log }} \rightarrow \widetilde{S}^{\text {log }}$ and some line bundle with connection $(\mathscr{L}$, $\nabla_{\mathscr{L}}$ ) whose square is trivial.

Note that this definition is consistent (though slightly weaker, since we allow the ambiguity of tensoring with $(\mathscr{L}, \nabla \mathscr{L})$ ) with the notion of being an object of the category $\mathcal{M} \mathscr{F}^{\nabla}$ of [Falt], §2. It is shown in [Falt] (Theorem 6.2) that the de Rham cohomology of a semistable family of varieties over $X^{\mathrm{log}}$ equipped with the Hodge filtration and the Gauss-Manin connection forms an object of $\mathcal{M} \mathscr{F}^{\nabla}$ (as long as $p-2$ is greater than or equal to the relative dimension of the family of varieties). The following result provides the link between what we are doing here and [Falt], $\$ 2$ :

Proposition 2.10. Let $\left(\mathscr{E}, \nabla_{\mathscr{E}}\right)$ be an indigenous bundle on $X^{\log }$. Then (E. $\nabla_{\mathscr{E}}$ ) forms an $\mathcal{M} \mathscr{F}^{\nabla}$-object on $X^{\text {-1og }}$ if and only if $\left(\mathscr{E}, \nabla_{\mathscr{E}}\right)$ is admissible and nilpotent.

Proof. First, let us assume that $\left(\mathscr{E}, \nabla_{\mathscr{E}}\right)$ forms an $\mathcal{M} \mathscr{F}^{\nabla}$-object on $X^{\text {log }}$ for the lifting $\widetilde{X}^{\mathrm{log}} \rightarrow \widetilde{S}^{\mathrm{log}}$. One computes easily from the definition of the connection $\nabla_{\Phi}$ that the $p$-curvature is nilpotent (with respect to the filtration $0 \oplus\left(\Phi * \mathscr{E} / F^{1}\right.$ $\left.(\mathscr{E})) \subseteq \Phi^{*} \widetilde{\mathscr{E}}\right)$. Also. (just as in the proof of Proposition 1.4), the derivative $\frac{1}{p} \Phi^{\prime}$ is of the form $(1+t)^{p-1}+f^{\prime}(t)$. Thus, since the Kodaira-Spencer morphism is the identity, it follows that the $p$-curvature (applied to $\partial \stackrel{\text { def }}{=} d / d t$ ) is obtained by multiplying a section of $\left(\Phi^{*} F^{1}(\mathscr{E})\right) \oplus 0$ by $\Phi^{-1}\left(\partial^{F}\right)$ times the $(p-1)$ th derivative of $(1+t)^{p-1}+f^{\prime}(t)$ (which is just -1 ) and regarding the result of this multiplication as a section of $0 \oplus\left(\Phi^{*} \mathscr{E} / F^{1}(\mathscr{E})\right)$. In particular. we see that the $p$-curvature is nonzero. Thus, $\left(\mathscr{E}, \nabla_{\mathscr{E}}\right)$ is admissible and nilpotent.

On the other hand, suppose that $\left(\mathscr{E}, \nabla_{\mathscr{E}}\right)$ is admissible and nilpotent. By the bijective correspondence of Proposition $2.5,\left(\mathscr{E}, \nabla_{\mathscr{E}}\right)$ corresponds to an FL-bundle $\left(\mathscr{G}, \nabla_{\mathscr{G}}\right)$. By Proposition 1.2, this FL-bundle corresponds to a lift-

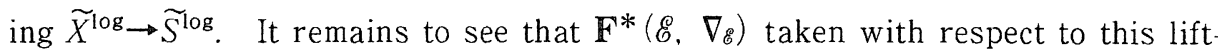
ing is isomorphic to $\left(\mathscr{E}, \nabla_{\mathscr{E}}\right)$ (up to tensoring with an $\left(\mathscr{L}, \nabla_{\mathscr{L}}\right)$ whose square is trivial). Let $\mathcal{N}=\mathscr{E} / F^{1}(\mathscr{E})$. Let $\mathcal{M}=\Phi_{X / S}^{*}\left(\mathcal{N}^{F}\right)$. Let $\nabla_{\mathcal{M}}$ be the connection on $\mathcal{M}$ for which the sections of $\mathcal{N}^{F}$ are horizontal. Thus, $\left(\mathcal{M}, \nabla_{\mathcal{M}}\right)^{\otimes 2} \cong\left(\mathscr{T}, \nabla_{\mathscr{T}}\right)$. Now, sorting through the definitions and using the fact that the Kodaira-Spencer morphism is the identity reveals that if $X=U \cup V$ (where $U$ and $V$ are affine opens $)$, then $\mathbf{F}^{*}\left(\mathscr{E}, \nabla_{\mathscr{E}}\right) \otimes\left(\mathcal{M}, \nabla_{\mathcal{M}}\right)$ is just the extension of $\mathscr{O}_{X}$ 
by $\mathscr{T}$ obtained from the 1-cocycle which is the difference between Frobenius liftings on $U$ and $V$. It thus follows from the definition of the canonical morphism $\mathscr{F}: \mathscr{D} \rightarrow \mathscr{A}$ of Proposition 1.2 that $\mathbb{F}^{*}\left(\mathscr{E}, \nabla_{\mathscr{E}}\right) \otimes\left(\mathcal{M}, \nabla_{\mathcal{M}}\right)$ is exactly the bundle $(\mathscr{G}, \nabla \mathscr{G})$. Thus, it follows from the definition of the bijective correspondence in Proposition 2.5 that $\mathbb{F}^{*}\left(\mathscr{E}, \nabla_{\mathscr{E}}\right)=\left(\mathscr{E}, \nabla_{\mathscr{E}}\right) \otimes\left(\mathscr{L}, \nabla_{\mathscr{L}}\right)$ for some $\left(\mathscr{L}, \nabla_{\mathscr{L}}\right)$ whose square is trivial.

\section{The Hnfinitesimal Verschiebung}

Let $\omega_{X / S}$ be the relative dualizing sheaf of $f: X \rightarrow S$. Thus, if $D \subseteq X$ is the divisor of marked points, we have $\omega_{X / S}(D) \cong \omega_{X / S}^{\log }$. Let $\Phi_{X / S}: X \rightarrow X^{F}$ be the relative Frobenius over $S$. Recall from duality theory (see, e.g., [Harts] for a treatment of duality theory) that since $f$ and $\Phi_{X / S}$ are local complete intersection morphisms, we have a trace morphism :

$$
\operatorname{tr}_{\Phi_{X, S}}:\left(\bar{\Phi}_{X / S}\right)_{*} \omega_{X / S} \rightarrow\left(\omega_{X / S}\right)^{F}
$$

where we regard $\omega_{X / S}$ as $\mathbb{R} \Phi_{X / S}^{\prime}\left(\omega_{X / S}\right)^{F}$. On the other hand, let $Y^{\text {tog }}$ be the log scheme whose underlying scheme is $X$ and whose log structure is the same as that of $X^{\mathrm{log}}$ away from the divisor $D$ of marked points, and equal to the pull-back of the log structure of $S^{\log }$ on the open subscheme where $f$ is smooth. Then we also have a morphism arising from the log version of the Cartier isomorphism (applied to $Y^{\log } \rightarrow S^{\log }$ ):

$$
C:\left(\Phi_{X / S}\right) * \omega_{X / S} \rightarrow\left(\omega_{X / S}\right)^{F}
$$

The following result is "well-known", but I do not know an adequate reference for it:

\section{Lemma 2.11. The morphisms $\operatorname{tr}_{\Phi_{x / s}}$ and $C$ are equal.}

Proof. By a density argument, we reduce to the case where $f$ is smooth. By naturality, we reduce to the assertion that these two morphisms are the same when $X=\operatorname{Spec}\left(\mathbb{F}_{p}[T]\right) ; S=\operatorname{Spec}\left(\mathbb{F}_{p}\right)$ (where $T$ is an indeterminate). Since $\operatorname{tr}_{\Phi_{x / s}}$ is the reduction modulo $p$ of a construction that holds in arbitrary or mixed characteristic, we can calculate $\operatorname{tr}_{\Phi_{x / s}}$ by considering the trace map obtained from duality for the finite morphism $\Phi:$ Spec $\left(\mathbb{Z}_{p}[T]\right) \rightarrow \operatorname{Spec}\left(\mathbb{Z}_{p}[T]\right)$ given by $T \rightarrow$ $T^{p}$. Since $\operatorname{tr}_{\Phi}\left(\Phi^{*}(d T)\right)=p d T$, and $\Phi^{*}(d T)=p T^{p-1} d T$, it follows that $\operatorname{tr}_{\Phi}\left(T^{p-1} d T\right)=d T$. By reducing this formula modulo $p$ and comparing with the construction in [Katz] of the Cartier isomorphism, we obtain the desired result.

Let $\left(\mathscr{E}, \nabla_{\mathscr{g}}\right)$ be an indigenous bundle on $X^{\text {log }}$. Let $H_{\mathscr{g}}: \mathscr{T} \rightarrow \tau_{X^{108} / S^{108}}$ be the 
square Hasse invariant of $\left(\mathscr{E}, \nabla_{\mathscr{E}}\right)$. Then by pulling back via $\Phi_{X^{108} / S^{108}}$ and taking $\mathbf{R}^{1} f_{*}$ of $H_{\&}$, we obtain a canonical morphism:

$$
\Phi_{g}^{\tau}:\left(\mathbf{R}^{1} f_{*} \tau_{X^{108} / S^{108}}\right) F \rightarrow \mathbf{R}^{1} f_{*} \tau_{X^{108} / S^{108}}
$$

which we shall call the Frobenius on $\mathbb{R}^{1} f_{*} \tau_{X^{108} / S^{108}}$ induced by $\left(\mathscr{E}, \nabla_{\mathscr{E}}\right)$. On the other hand. let us consider the dual morphism to $H_{\mathscr{E}}$, that is, $H_{\mathscr{E}}^{\vee}: \omega_{X / S}^{\log } \rightarrow \mathscr{T}^{\vee}$. Note that $\mathscr{T}^{\vee}=\Phi_{X / S}^{*}\left(\omega_{X / S}^{\log }\right)^{F}$. Thus, if we tensor $H_{\mathscr{g}}^{\vee}$ with $\omega_{X / S}$, we get a morphism

$$
\left(\omega_{X / S}^{\log }\right)^{\otimes 2}(-D) \rightarrow \omega_{X / S} \otimes_{\mathscr{O}_{X}} \Phi_{X / S}^{*}\left(\omega_{X / S}^{\log }\right)^{F}
$$

If we then compose this morphism with the trace morphism $\operatorname{tr}_{\Phi_{X / S}}$ tensored with $\left(\omega_{X / S}^{\log }\right)^{F}$, we obtain a morphism $\left(\Phi_{X / S}\right) *\left(\omega_{X / S}^{\log }\right)^{\otimes 2}(-D) \rightarrow\left(\left(\omega_{X / S}^{\log }\right)^{\otimes 2}(-D)\right)^{F}$. Then applying $f_{*}$ to this morphism. we obtain:

$$
\Phi_{\mathscr{E}}^{\omega}: f_{*}\left(\omega_{X / S}^{\log }\right) \otimes 2(-D) \rightarrow\left(f_{*}\left(\omega_{X / S}^{\log }\right) \otimes 2(-D)\right)^{F}
$$

which we shall call the Verschiebung on $f_{*}\left(\omega_{X / S}^{\log }\right) \otimes 2(-D)$ induced by $\left(\mathscr{E}, \nabla_{\mathscr{E}}\right)$. Observe that by Serre duality applied to the morphism $f$, we obtain that the vector bundles $f_{*}\left(\omega_{X / S}^{\log }\right) \otimes 2(-D)$ and $\mathbb{R}^{1} f_{*} \tau_{X^{108} / S^{108}}$ on $S$ are dual to one another. Then relative to this duality, we have

\section{Proposition 2.12. The morphisms $\Phi_{\&}^{\omega}$ and $\Phi_{g}^{\tau}$ are dual to one another.}

Proof. This follows immediately from duality theory. Namely, the duality between $f_{*} \omega_{X / S}^{\otimes 2}(-D)$ and $\mathbb{R}^{1} f_{*} \tau_{X^{108} / S^{108}}$ is obtained by cup product, followed by the trace morphism $\operatorname{tr}_{f}: \mathbb{R}^{1} f_{*} \omega_{X / S} \rightarrow \mathbb{O}_{S}$. On the other hand, since trace morph isms behave well under composition, we see that $\operatorname{tr}_{f}=\left(\operatorname{tr}_{f}\right)^{F}: \operatorname{tr}_{\Phi_{x / s}}$. This fact. combined with the fact that $\Phi_{g}^{\tau}$ (respectively. $\Phi_{g}^{\omega}$ ) is obtained from $H_{\mathscr{E}}$ (respectively, $H_{\mathscr{E}}^{\vee}$ ), implies the result.

We are now ready to state and prove the second main result of this Section. Consider the Verschiebung on indigenous bundles defined in the first subsection of this Section. In the universal case, it was a morphism

$$
\overline{\mathcal{V}}_{g, r}: \overline{\mathscr{Q}}_{g, r} \rightarrow \overline{\mathscr{Q}}_{g, r}
$$

over $\bar{M}_{g, r}$ Thus, it induces a map on tangent bundles over $\bar{M}_{g, r}$ :

$$
\Theta_{\bar{V}_{0}, / \bar{M}_{\theta},}: \Theta_{\bar{s}_{\theta}, / \bar{t}_{0},} \rightarrow \Theta_{\bar{Q}_{\theta}, / \bar{M}_{a}}
$$

If we pull-back to the point of $\overline{\mathscr{\varnothing}}_{g, r}$ defined by our particular $\left(\mathscr{E}, \nabla_{\mathscr{E}}\right)$, we get a morphism 


$$
\Theta_{g}^{V}: f_{*}\left(\omega_{X / S}^{\log }\right) \otimes 2(-D) \rightarrow\left(f_{*}\left(\omega_{X / S}^{\log }\right) \otimes 2(-D)\right)^{F}
$$

Then we have the following result:

Theorem 2.13. Let $\left(\mathscr{E}, \nabla_{\mathscr{B}}\right)$ be indigenous on $X^{\log }$. Then the infinitesimal Verschiebung morphism $\Theta_{\mathscr{E}}^{V}$ is equal to $\Phi_{\mathscr{E}}^{\omega}$.

Proof. First, let us recall Jacobson's formula (see, e.g., [Jac]. pp.186-187): This formula states that if $a$ and $b$ are elements of an associative ring $R$ of characteristic $p$, then

$$
(a+b)^{p}=a^{p}+b^{p}+\sum_{i=1}^{p-1} s_{i}(a, b)
$$

where the $s_{i}(a, b)$ are given by the formula:

$$
(\operatorname{ad}(t a+b))^{p-1}(a)=\sum_{i=1}^{p-1} i s_{l}(a, b) t^{\imath-1}
$$

computed in the ring $R[t]$, where $t$ is an indeterminate. In our case, we wish to apply this formula in the case where $a=\epsilon \alpha, \alpha \in R$, and $\epsilon$ is an element of the center of $R$ such that $\epsilon^{2}=0$. In this case, substitution yields:

$$
(a+b)^{p}=b^{p}+(\operatorname{ad}(b))^{p-1} a
$$

To prove that $\Theta_{\mathscr{g}}^{*}=\Phi_{\mathscr{g}}^{\omega}$, it suffices to do a local calculation on $X$ to show that the infinitesimal change in the trace of the square of the $p$-curvature is given by $-2 \Phi_{g}^{\omega}$. We work over the base $S[\epsilon] /\left(\epsilon^{2}\right)$. Let $\nabla_{\xi}^{\prime}=\nabla_{\varepsilon}+\epsilon \theta$, where $\theta$ is a section of $f_{*}\left(\omega_{X / S}^{\log }\right)^{\otimes 2}(-D)$, be a connection on $\mathscr{E}$ that makes it an indigenous bundle. Let $U \subseteq X$ be an open subset that avoids the marked points and at which $f$ is smooth. Let $x$ be a local coordinate on $U$. Write $\nabla$ (respectively, $\left.\nabla^{\prime} ; \theta_{x}\right)$ for $\nabla_{\mathscr{E}}$ (respectively, $\left.\nabla_{\mathscr{g}}^{\prime} ; \theta\right)$ applied in the direction $\partial \stackrel{\text { def }}{=} \frac{d}{d x}$. We wish to apply the above formula in the case where $b=\nabla$ and $a=\epsilon \theta_{x}$. We thus obtain that the infinitesimal change in the p-curvature is given by $(\operatorname{ad}(\nabla))^{p-1} \theta_{x}$. Now the infinitesimal Verschiebung is obtained by multiplying this term by the constant term and then taking the trace (and multiplying by -1). Put another way, minus the infinitesimal Verschiebung is obtained by applying $\mathscr{P}_{g}^{\vee}$ to $(\operatorname{ad}(\nabla))^{p-1} \theta_{x}$. Since $\mathscr{P}_{g}^{\vee}$ is horizontal, it commutes with ad $(\nabla)$, so we find that:

$$
(\partial)^{p-1}\left(\mathscr{P}_{\mathscr{E}}^{\vee} \theta_{x}\right)=(\partial)^{p-1}\left(\left(\mathscr{P}_{\mathscr{E}}^{\vee} \theta\right) \cdot \partial\right)
$$


is the section of $\Phi_{X / S}^{*}\left(\omega_{X / S}^{\log }\right)^{F}$ obtained by evaluating minus the infinitesimal Verschiebung (which is a section of $\left.\Phi_{X / S}^{*}\left\{\left(\left(\omega_{X / S}^{\log }\right){ }^{\otimes 2}\right)(-D)\right\}^{F}\right)$ at $\partial^{F}$. Thus, to complete the proof of the Theorem, we must show that

$$
(\partial)^{p-1}\left(\left(\mathscr{P}_{\mathscr{g}}^{\vee} \theta\right) \cdot \partial\right)=-\Phi_{X / S}^{-1}\left\{(C \otimes 1)\left(\mathscr{P}_{g}^{\vee} \theta\right) \cdot \partial^{F}\right\}
$$

Now let $\zeta$ be a horizontal, locally generating section of $\Phi_{X / S}^{*}\left(\omega_{X / S}^{\log }\right)^{F}$. Write $\mathscr{P}_{g}^{\vee} \theta=\nu \cdot \zeta$, where $\nu$ is a section of $\omega_{X / s}$. Substituting, we see that it suffices to show that:

$$
(\partial)^{p-1}(\nu \cdot \partial) \cdot \zeta=-\Phi_{X / S}^{-1}\left(C(\nu) \cdot \partial^{F}\right) \cdot \zeta
$$

If we then divide out the $\zeta$ 's, we see that really (just as in [Katz2], (7.1.2.6)), we are reduced to proving a simple identity concerning differentiation in characteristic $p$. Indeed, if we regard the equation as an identity in $\nu$, both sides are $\Phi_{X / S}^{-1} \mathscr{O}_{X^{F}}$-linear in $\nu$ and vanish when $\nu$ is exact; thus, we are reduced to proving the identity :

$$
(\partial)^{p-1}\left(x^{p-1}\right)=-C\left(x^{p-1} d x\right) \cdot \partial^{F}
$$

which follows from the definition of $C$ and the fact that $(p-1) !=-1$ in characteristic $p$.

\section{Differential Criterion for Admissibility}

We maintain the notation of the previous subsection. In the previous subsection, we computed the relative differential map of the Verschiebung morphism $\bar{V}_{g, r}$ over $\bar{M}_{g, r}$. In fact, however, with a little more preparation, the same calculation allows us to give an explicit representation of the differential map of the Verschiebung over $\mathbb{F}_{p}$. Moreover, this explicit representation allows us to give a differential criterion for an indigenous bundle to be admissible, which is also necessary if the bundle is nilpotent.

Consider the affine bundle $\overline{\mathscr{Q}}_{g, r} \rightarrow \bar{M}_{g, r}$. Since by definition, this bundle is the pull back by the Frobenius morphism on $\bar{M}_{g, r}$ of a bundle over $\bar{M}_{g, r}$, it follows that we get a natural connection $\nabla_{\mathscr{Q}}$ on this affine bundle $\overline{\mathscr{Q}}_{g, r} \rightarrow \bar{M}_{g, r}$. Let $\bar{Q}_{g, r}^{\log }$ be the log stack obtained by pulling back to $\overline{\mathscr{Q}}_{g, r}$ the log structure of $\overline{\mathbb{M}}_{g, r}^{\text {log }}$. Next. let us consider the canonical exact sequence of tangent bundles on $\overline{\mathscr{Q}}_{g, r}$ :

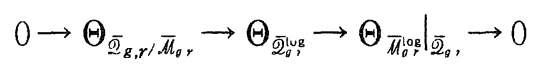

Thus, our connection $\nabla_{\mathscr{Q}}$ induces a splitting

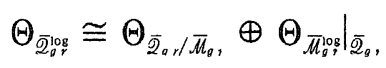


Now let us consider the "full" infinitesimal Verschiebung, i.e., the morphism induced on tangent bundles by $\overline{\mathscr{V}}_{g, r}$ :

$$
\Theta_{\bar{V}_{a r}}: \Theta_{\bar{\Sigma}_{g r}} \rightarrow \Theta_{\bar{Q}_{g r}}
$$

On the one hand, we know what the projection of $\Theta_{\bar{V}_{g, r}}$ to $\Theta \overline{\pi t i o g}_{i_{r}} \overline{\mathscr{Q}}_{g, r}$ is. Thus, we would like to compute the projection of $\Theta_{\bar{V}_{g, r}}$ to $\Theta_{\bar{Q}_{g, r} / \bar{H}_{g, r}}$. We shall soon see that, in fact, we have already computed this projection as well, in the course of proving Theorem 2.13.

Suppose (as in the previous subsection) we have a log scheme $S^{\log }$ with an $r$-pointed stable curve of genus $g, f^{\log }: X^{\log } \rightarrow S^{\log }$, and an indigenous bundle $(\mathscr{E}$, $\left.\nabla_{\&}\right)$ on $X^{\log }$. Then the first relative parabolic de Rham cohomology module $\mathbb{R}^{1} f_{\mathrm{DR}, *}(\mathrm{Ad}(\mathscr{E})$ ) (as in Chapter I, Theorem 2.8) is naturally isomorphic to the

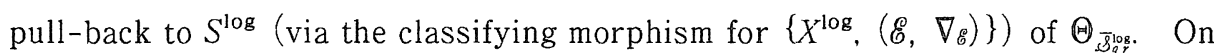
$\mathbb{R}^{1} f_{\mathrm{DR}, *}(\operatorname{Ad}(\mathscr{E}))$, we have a Hodge filtration

$$
0 \rightarrow f_{*}\left(\omega_{X / S}^{\log }\right){ }^{\otimes 2}(-D) \rightarrow \mathbb{R}^{1} f_{\mathrm{DR}, *}(\operatorname{Ad}(\mathscr{E})) \rightarrow \mathbb{R}^{1} f_{*} \tau_{X^{108} / S^{\log }} \rightarrow 0
$$

where the surjection in the above exact sequence is exactly the pull-back to $S^{\log }$

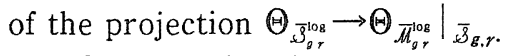

On the other hand, consider the $p$-curvature of $\operatorname{Ad}(\mathscr{E}): \mathscr{P}_{\mathscr{E}}: \mathscr{T} \rightarrow \operatorname{Ad}(\mathscr{E})$. Then by applying " $\mathbb{R}^{1} f_{\mathrm{DR}, *}$ " to the dual of $\mathscr{P}_{\mathscr{E}}$, we get a morphism

$$
\mathbb{R}^{1} f_{\mathrm{DR}, *}(\operatorname{Ad}(\mathscr{E})) \rightarrow \mathbb{R}^{1} f_{\mathrm{DR}, *}\left(\mathscr{T}^{\vee}\right)
$$

Now by Poincaré duality, we have

$$
\mathbb{R}^{1} f_{\mathrm{DR}, *}\left(\mathscr{T}^{\vee}\right) \cong\left\{\mathbb{R}^{1} f_{\mathrm{DR}, *}(\mathscr{T})\right\}^{\vee}
$$

Moreover, we computed $\mathbb{R}^{1} f_{\mathrm{DR}, *}(\mathscr{T})$ in Proposition 1.1. In particular, we have a natural inclusion $\mathbb{R}^{1} f_{*}\left(\tau_{X^{108} / S^{108}}\right) \subseteq C \mathbb{R}^{1} f_{\mathrm{DR}, *}(\mathscr{T})$. Thus, if we compose the above morphism induced by $\mathscr{P}_{\mathscr{E}}^{\vee}$ with the dual surjection to this natural inclusion, we obtain a natural morphism

$$
\Theta_{\mathscr{E}}: \mathbb{R}^{1} f_{\mathrm{DR}, *}(\operatorname{Ad}(\mathscr{E})) \rightarrow\left(f_{*}\left(\omega_{X / S}^{\log }\right)^{\otimes 2}(-D)\right)^{F}
$$

Then we have the following result :

Theorem 2.14. Let $\left(\mathscr{E}, \nabla_{\mathscr{E}}\right)$ be indigenous on $X^{\mathrm{log}}$. Then the pull-back to $S^{\log }$ of the projection of $\Theta \bar{v}_{g, r}$ to $\Theta \bar{M}_{g}^{108}$ is given by the surjection in the Hodge filtration; the pull-back to $S^{\log }$ of the projection of $\Theta_{\bar{r}_{\theta, r}}$ to $\Theta_{\bar{Q}_{g, r} / \bar{H}_{g, r}}$ is given by $\Theta_{\delta}$.

Proof. It remains to prove the statement about the projection to $\Theta \overline{\mathscr{Q}}_{g, r} / \bar{M}_{g, r}$. To do this we consider an infinitesimal deformation of $\left(\mathscr{E}, \nabla_{\mathscr{E}}\right)$ over $S^{\log }[\epsilon] /$ 
$\left(\epsilon^{2}\right)$. But the section of $\left(f_{*}\left(\omega_{X / S}^{\log }\right)^{\otimes 2}(-D)\right)^{F}$ that we obtain can be computed locally on $X$. Moreover, locally on $X$, this calculation is exactly the same as that of Theorem 2.13. This proves the result.

Corollary 2.15. Let $\left(\mathscr{E}, \nabla_{\mathscr{E}}\right)$ be indigenous on $X^{\mathrm{log}}$. If $\Theta_{\mathscr{E}}$ is surjective, then $\left(\mathscr{E}, \nabla_{\mathscr{E}}\right)$ is admissible. In particular, if the morphism $\overline{\mathscr{V}}_{g, r}^{\log }: \overline{\mathscr{X}}_{g, r}^{\log } \rightarrow \overline{\mathscr{Q}}_{g, r}^{\log }$ is log étale

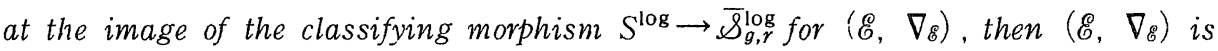
admissible.

Proof. Since being admissible is an open condition, it suffices to prove the result when $S$ is the spectrum of an algebraically closed field. If $\overline{\mathscr{V}}_{\boldsymbol{g}, \boldsymbol{r}}^{\mathrm{log}}$ is $\log$ étale, then by Theorem $2.14, \Theta_{g}$ must be surjective. Thus, it suffices to prove the first statement. Suppose that $\left(\mathscr{E}, \nabla_{\mathscr{E}}\right)$ is not admissible. Thus, the morphism $\mathscr{P}_{\mathscr{E}}^{\vee}: \operatorname{Ad}(\mathscr{E}) \rightarrow \mathscr{T}^{\vee}$ has a nonempty zero locus. One can compute the $p$-curvature explicitly at a marked point (where the monodromy is nonzero and nilpotent); it follows that the zero locus does not contain any marked points. Now there are two possibilities: the zero locus either avoids the nodes or it does not.

Let us first do the case where the zero locus of $\mathscr{P}_{\mathscr{E}}^{v}$ avoids the nodes. Since $\mathscr{P}_{\mathscr{B}}^{\vee}$ is horizontal, its zero locus must be the pull-back of some closed subscheme via $\Phi_{X / s}$. Thus, in particular, there exists some point $x \in X$ (which is neither a marked point nor a node) such that $\mathscr{P}_{\mathscr{G}}^{\vee}$ is zero at $\Phi_{X / S}^{-1}\left(x^{F}\right)$ (the scheme-theoretic fiber). By the definition of $\Theta_{\mathscr{g}}$, it follows that the image of $\Theta_{\mathscr{g}}$ lands in the subspace $V_{x}$ of $H^{0}\left(X,\left(\omega_{X / S}^{\log }\right)^{\otimes 2}(-D)\right)^{F}$ consisting of sections that vanish at $x^{F}$. Now by Riemann-Roch on curves, $V_{x}$ cannot be all of $H^{0}(X$, $\left.\left(\omega_{X / S}^{\log }\right)^{\otimes 2}(-D)\right)^{F}$, unless $g=0$ and $r=3$, or $g=1$ and $r=1$. This completes the proof (under the assumption that $\mathscr{P}_{\mathscr{g}}^{v}$ avoids the nodes), except for these two special cases. For $g=0, r=3$, we shall show in the subsection of $\S 3$ on totally degenerate curves that the unique indigenous bundle on such a curve is necessarily admissible. (One checks easily that there are no vicious circles in the reasoning.) For $g=1, r=1$, we note that $\mathbb{P}\left(\mathscr{E}, \nabla_{\mathscr{E}}\right)$ is necessarily invariant with respect to the automorphism $\alpha$ given by multiplying by -1 . Thus, if we pull-back by the morphism $X \rightarrow X$ given by multiplying by 2 , it is still invariant under $\alpha$. Hence it descends to the four-pointed curve of genus zero $Y^{\text {log }}$ of which $X^{\log }$ is a $\log$ étale double covering. Let us call this descended bundle $\mathbb{P}\left(\mathscr{F}, \nabla_{\mathscr{F}}\right)$. Thus, $\mathbf{P}\left(\mathscr{F}, \nabla_{\mathscr{F}}\right)$ is indigenous on $Y^{\mathrm{log}}$. It is easy to see that $\Theta_{\mathscr{E}}$ and $\Theta_{\mathscr{F}}$ are the same morphism; thus, the hypothesis holds for $\mathbf{P}\left(\mathscr{F}, \nabla_{\mathscr{F}}\right)$, as well. Thus, we reduce to the case $g=0, r=4$, which has already been checked.

Now let us consider the case where $\mathscr{P}_{\tilde{\delta}}^{v}$ vanishes at a node $\nu \in X$. Let $\pi^{\text {log. }}$ 
$Z^{\log } \rightarrow X^{\log }$ be the partial normalization of $X^{\log }$ at $\nu$ (where the log structure on $Z^{\log }$ is such that the points mapping to $\nu$ are marked points). Let (FF, $\nabla_{\mathscr{F}}$ ) $\stackrel{\text { def }}{=} \pi^{*}\left(\mathscr{E}, \nabla_{\mathscr{E}}\right)$. Now let us consider the commutative diagram :

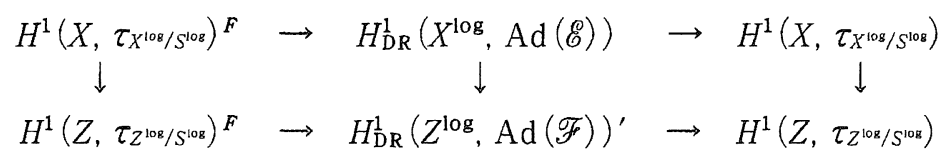

where the vertical arrows are pull-backs via $\pi$; the horizontal arrows on the left are induced by $\mathscr{P}_{g}$; and the horizontal arrows on the right are induced by the Hodge filtration. Finally, the "prime" on the de Rham cohomology on the bottom row indicates that the we are taking non-parabolic de Rham cohomology on $Z^{\text {log }}$ (since $\left(\mathscr{F}, \nabla_{\mathscr{F}}\right)$ may not even have a natural parabolic structure, if $(\mathscr{E}$, $\left.\nabla_{\mathscr{E}}\right)$ is not of restrictable type). Let $\eta$ be a generator of the kernel of $H^{1}(X$, $\tau_{\left.X^{108} / S^{108}\right)} \rightarrow H^{1}\left(Z, \tau_{Z^{108} / S^{108}}\right)$. Let us consider what happens to $\eta^{F}$ as we move it around the above commutative diagram. Since $\mathscr{P}_{\mathscr{g}}$ is zero at $\nu$, if we move it along the top to $H^{1}\left(X, \tau_{X^{108} / S^{108}}\right)$. we get zero. Thus, its image in $H_{\mathrm{DR}}^{1}\left(X^{\text {log }}\right.$, $\operatorname{Ad}(\mathscr{E}))$ lies in $H^{0}\left(X,\left(\omega_{X / S}^{\log }\right) \otimes 2(-D)\right)$. But the pull-back map $H_{\mathrm{DR}}^{1}\left(X^{\log }, \operatorname{Ad}(\mathscr{E})\right)$ $\rightarrow H_{\mathrm{DR}}^{1}\left(Z^{\log }, \operatorname{Ad}(\mathscr{F})\right)^{\prime}$ maps $H^{0}\left(X,\left(\omega_{X / S}^{\log }\right) \otimes 2(-D)\right)$ injectively into $H^{0}(Z$, $\left.\left(\omega_{X / S}^{\log }\right)^{\otimes 2}\right)$. Going around the other way. however, (i.e., going down and then to the right), we see that the image of $\eta^{F}$ in $H_{\mathrm{DR}}^{1}\left(Z^{\log }, \mathrm{Ad}(\mathscr{F})\right)^{\prime}$ is zero. We thus conclude that the image of $\eta^{F}$ in $H_{\mathrm{DR}}^{1}\left(X^{\log }, \mathrm{Ad}(\mathscr{E})\right)$ is zero. But this contradicts the surjectivity of $\Theta_{\delta}$. since the upper horizontal morphism on the left-hand side of the above diagram is dual to $\Theta_{\varepsilon}$. This completes the proof.

Conversely, let us suppose that $\left(\mathscr{E}, \nabla_{\mathscr{E}}\right)$ is nilpotent and admissible. Then we claim that $\Theta_{\mathscr{E}}$ is necessarily surjective. Indeed, for simplicity, it suffices to prove this when $S$ is the spectrum of a field. Let $\mathscr{K}$ be the kernel of $\mathscr{P}_{\mathscr{B}}^{\vee}$ : $\operatorname{Ad}(\mathscr{E}) \rightarrow \mathscr{T}^{\vee}$. Note that the connection on $\operatorname{Ad}(\mathscr{E})$ restricts to a connection $\nabla_{\mathscr{H}}$ on $\mathscr{K}$. Thus, $\left(\mathscr{K}, \nabla_{\mathscr{K}}\right)$ is an FL-bundle. Also, we have a horizontal exact sequence :

$$
0 \rightarrow \mathscr{T} \rightarrow \mathscr{K} \rightarrow \mathscr{O}_{X} \rightarrow 0
$$

in which the connecting morphism $\mathscr{O}_{s} \rightarrow \mathbb{R}^{1} f_{\mathrm{DR}, *}(\mathscr{T})$ must be injective (since $(\mathscr{K}$, $\left.\nabla_{\mathscr{H}}\right)$ is an FL-bundle). Moreover, it is a tautology that the composite of this morphism $\mathscr{O}_{S} \rightarrow \mathbb{R}^{1} f_{\mathrm{DR}, *}(\mathscr{T})$ with the projection $\mathbb{R}^{1} f_{\mathrm{DR}, *}(\mathscr{T}) \rightarrow \mathscr{O}_{S}$ of Proposition 1.1 is the identity. Thus, we see that the morphism $\mathbb{R}^{1} f_{*}\left(\tau_{X^{108} / S^{108}}\right)^{F} \longrightarrow \mathbb{R}^{1} f_{\mathrm{DR}, *}$ $(\mathscr{T}) \rightarrow \mathbb{R}^{1} f_{\mathrm{DR}, *}(\mathscr{K})$ is injective. Now let $\mathscr{C}=\operatorname{Ad}(\mathscr{E}) / \mathscr{T}$. We have a connection $\nabla_{\mathscr{C}}$ on $\mathscr{C}$, induced by the connection on $\operatorname{Ad}(\mathscr{E})$. Thus, we get a horizontal exact sequence : 


$$
0 \rightarrow \mathscr{O}_{X} \rightarrow \mathscr{C} \rightarrow \mathscr{T}^{\vee} \rightarrow 0
$$

in which the connecting morphism $\left(f_{*} \omega_{X / S}^{\log }\right)^{F} \rightarrow \mathbb{R}^{1} f_{\mathrm{DR}, *}\left(\mathscr{O}_{X}\right)$ must be injective (since otherwise, $\mathscr{C}$ would admit two horizontal, generically linearly indendent sections, which contradicts the fact that the $p$-curvature is nonzero). Lastly, we consider the horizontal exact sequence:

$$
0 \rightarrow \mathscr{K} \rightarrow \operatorname{Ad}(\mathscr{E}) \rightarrow \mathscr{T}^{\vee} \rightarrow 0
$$

in which the composite of the connecting morphism $\left(f_{*} \omega_{X / S}^{\log }\right)^{F} \rightarrow \mathbb{R}^{1} f_{\mathrm{DR}, *}(\mathscr{K})$ with the projection $\mathbb{R}^{1} f_{\mathrm{DR}, *}(\mathscr{K}) \rightarrow \mathbb{R}^{1} f_{\mathrm{DR}, *}\left(\mathscr{O}_{X}\right)$ is injective, as we observed above. Since the image of $\mathbb{R}^{1} f_{*}\left(\tau_{X^{108} / S^{108}}\right)^{F} \subseteq \mathbb{R}^{1} f_{\mathrm{DR}, *}(\mathscr{K})$ under the map $\mathbb{R}^{1} f_{\mathrm{DR}, *}(\mathscr{K}) \rightarrow$ $\mathbb{R}^{1} f_{\mathrm{DR}, *}\left(\mathscr{O}_{X}\right)$ is zero, it thus follows that if we compose the inclusion $\mathbb{R}^{1} f_{*}$ $\left(\tau_{Y^{108} / S^{108}}\right)^{F} \subseteq \mathbb{R}^{1} f_{\mathrm{DR}, *}(\mathscr{K})$ with the morphism $\mathbb{R}^{1} f_{\mathrm{DR}, *}(\mathscr{K}) \rightarrow \mathbb{R}^{1} f_{\mathrm{DR}, *}(\operatorname{Ad}(\mathscr{E}))$, the resulting morphism

$$
\mathbb{R}^{1} f_{*}\left(\tau_{\left.\lambda^{108} / S^{108}\right)}^{F} \rightarrow \mathbb{R}^{1} f_{\mathrm{DR}, *}(\operatorname{Ad}(\mathscr{E}))\right.
$$

is injective. But this morphism is dual to $\Theta_{8}$. Thus, $\Theta_{8}$ is surjective. This completes the proof of the claim.

Let $\overline{\mathcal{N}}_{g . r}^{\text {log }}$ be the log stack obtained by pulling back the log structure on $\bar{M}_{g, r}^{\log }$ to $\overline{\mathcal{N}}_{g, r}$. Since $\overline{\mathcal{N}}_{g, r}$ is the zero locus of the Verschiebung, it follows that $\overline{\mathcal{N}}_{g, r}^{\log }$ is $\log$ smooth over $\mathbb{F}_{p}$ at a point if and only if $\Theta_{\mathcal{S}}$ is surjective. In other words :

Corollary 2.16. Suppose that $\left(\mathscr{E}, \nabla_{\mathscr{E}}\right)$ is nilpotent indigenous. Then it is admissible if and only if $\overline{\mathcal{N}}_{g, r}$ is smooth over $\mathbf{F}_{p}$ at the image of classifying morphism $S^{\log } \rightarrow \overline{\mathcal{N}}_{g, r}^{\log }$ for $\left(\mathscr{E}, \nabla_{\mathscr{E}}\right)$.

\section{§3. Hyperbolically Ordinary Curves}

Often, in the literature, one speaks of a curve as being "ordinary" if its Jacobian is ordinary. In fact, however, since the Jacobian only represents the "abelian part" of the curve, it is in some sense more intrinsic to speak of a curve as ordinary if it is hyperbolically ordinary in the sense defined below. Philosophically, this means that the Verschiebung on indigenous bundles is a local isomorphism in a neighborhood of an indigenous bundle that provides a "nice" uniformization for the curve. Thus, relative to the analogy (explained in the Introduction) between the Verschiebung on indigenous bundles and the Beltrami equation, to be hyperbolically ordinary means that the Verschiebung acts (at least locally) as one might expect from this analogy, given the classical results 
on existence and uniqueness of solutions to the Beltrami equation.

\section{Basic Definitions}

Let $S^{\log }$ be a fine noetherian log scheme over $\mathbb{F}_{p}$. Let $f^{\log }: X^{\log } \rightarrow S^{\log }$ be an $r$-pointed stable curve of genus $g$ (so $2 g-2+r \geq 1$ ). Let $D \subseteq X$ be the divisor of marked points. Let $\left(\mathscr{E}, \nabla_{\mathscr{B}}\right)$ be an indigenous bundle on $X^{\log }$. Let $\mathscr{P}_{\mathscr{g}}: \mathscr{T} \rightarrow \operatorname{Ad}(\mathscr{E})$

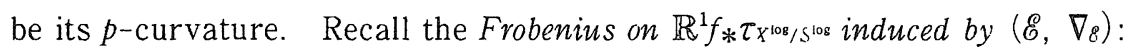

$$
\Phi_{\mathscr{E}}^{\tau}:\left(\mathbb{R}^{1} f_{*} \tau_{X^{108} / S^{108}}\right)^{F} \rightarrow \mathbb{R}^{1} f_{*} \tau_{X^{108} / S^{108}}
$$

that we defined in $\S 2$.

Definition $\mathfrak{B}_{0} \mathbb{1}$. We shall call $\left(\mathscr{E}, \nabla_{\mathscr{E}}\right)$ ordinary if $\Phi_{\mathscr{E}}^{\tau}$ is an isomorphism.

Note that the condition of being ordinary is an open condition on $\overline{\mathscr{\varnothing}}_{g, r}$. We shall denote this open set by $\overline{\mathscr{\varnothing}}_{g, r}^{\text {ord }}$.

Proposition 3.2. If $\left(\mathscr{E}, \nabla_{\mathscr{E}}\right)$ is ordinary, then it is admissible.

Proof. This follows a fortiori from Corollary 2.15. $\bigcirc$

The following definition is key to the entire paper :

Definition 3.3. We shall say that $f^{\log }: X^{\log } \rightarrow S^{\log }$ is a hyperbolically ordinary curve if there exists an étale surjection $T \rightarrow S$ and a nilpotent, ordinary indigenous bundle $\left(\mathscr{E}, \nabla_{\mathscr{E}}\right)$ on $X^{\mathrm{log}} \times{ }_{S} T$.

When the context is clear, we shall simply say that $f^{\text {log }}$ is an "ordinary curve." The reason for the descriptive "hyperbolically" is that in the literature, the term "ordinary curve" is frequently used to mean that its Jacobian is ordinary. In this paper, when $f^{\text {log }}$ has an ordinary Jacobian. we shall say that $f^{\text {log }}$ is parabolically ordinary.

Proposition 3.4. If the fiber of $f^{\log }: X^{\log } \rightarrow S^{\log }$ over $s \in S$ is hyperbolically ordinary, then there exists an open set $U \subseteq S$ such that $\left.f^{\mathrm{log}}\right|_{U}$ is hyperbolically ordinary. In particular, $f^{\mathrm{log}}: X^{\mathrm{log}} \rightarrow S^{\mathrm{log}}$ is hyperbolically ordinary if and only if all its $f i$. bers are hyperbolically ordinary.

Proof. Indeed, it suffices to consider the universal example. Recall that $\overline{\mathcal{N}}_{g, r} \subseteq \overline{8}_{g, r}$ is the locus of nilpotent indigenous bundles. Write $\pi: \overline{\mathcal{N}}_{g, r} \rightarrow \bar{M}_{g, r}$ 
for the natural projection. Let $n \in \overline{\mathcal{N}}_{g, r}$; let $m \in \bar{M}_{g, r}$ be the point $\pi(n)$. Then it follows from Theorem 2.13, plus the definition of $\overline{\mathcal{N}}_{g, r}$ as the zero locus of $\bar{V}_{g, r}$ that if $n$ is ordinary, then $\pi$ must be étale at $n$. Thus, $\pi$ is open at $n$. This completes the proof.

We shall denote the open subscheme of $\bar{M}_{g, r}$ (respectively, $\overline{\mathcal{N}}_{g, r}$ ) consisting of hyperbolically ordinary curves (respectively, nilpotent, ordinary indigenous bundles) by $\overline{\mathcal{M}}_{g, r}^{\text {ord }}$ (respectively, $\overline{\mathcal{N}}_{g, r}^{\text {ord }}$ ). Thus, we have a natural étale surjec. tion

$$
\pi: \overline{\mathcal{N}}_{g, r}^{\text {ord }} \rightarrow \overline{\mathcal{M}}_{g . r}^{\text {ord }}
$$

Finally, let us note that over $\overline{\mathscr{S}}_{g, r}^{\text {ord }}$, we have an étale local system in $\mathbb{F}_{p}$-vector spaces of dimension $3 g-3+r$ obtained by taking the sections of

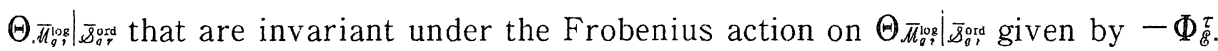
(Note the minus sign in front of $-\Phi_{\mathscr{E}}^{\tau}$ ! It will be important later in Chapter IJI.) Let us denote this local system by $\Theta_{g, r}^{\mathrm{et}}$, and call it the tangential local system on $\overline{\mathscr{S}}_{g, r}^{\text {ord }}$. Similarly, by taking its dual $\Omega_{g, r}^{\text {et }}$ we obtain a local system on $\overline{\mathscr{S}}_{g, r}^{\text {ord }}$ which we shall call the differential local system on $\overline{\mathscr{\bigotimes}}_{g, r}^{\text {ord }}$. Often, we shall be interested in the restrictions of these local systems to $\overline{\mathcal{N}}_{g, r}$.

\section{The Totally Degenerate Case}

In this subsection, we show that totally degenerate curves are hyperbolically ordinary. By Proposition 3.4, this will show that $\overline{\mathcal{M}}_{g, r}^{\text {ord }}$ is an open dense subscheme of $\bar{M}_{g, r}$. Since totally degenerate curves have no moduli, there is no loss of generality in assuming that $S=\operatorname{Spec}\left(\mathbf{F}_{p}\right)$. We begin by considering the case $g=0, r=3$. Recall the morphisms constructed at the end of Chapter I, $\S 3$ :

$$
N^{\log }: \bar{M}_{1,1}^{\log }[2] \rightarrow \bar{M}_{1,1}
$$

(parametrizing elliptic curves with level structures on the two-torsion points) and

$$
\Lambda^{\log }: \overline{\mathscr{M}}_{1,1}^{\log }[2] \rightarrow \bar{M}_{0,4}^{\log }
$$

which takes an elliptic curve to the four-pointed curve of genus zero of which it is a double covering. Then in this case, we have $\bar{M}_{0,4}^{\log }=X^{\log }$. Let us construct a nilpotent, admissible indigenous bundle on $\bar{M}_{0,4}^{\log }$. Since $X^{\log }$ has only one indigenous $\mathbf{P}^{1}$-bundle (up to isomorphism), this will complete the proof of Corollary 2.15. To do this, we note that (as we saw in Example 3 of Chapter I, §2), the first de Rham cohomology module of the universal elliptic curve over 
$\bar{M}_{1,1}^{\log }[2]$ defines an indigenous vector bundle $\left(\mathscr{E}, \nabla_{\mathscr{E}}\right)$. Let $\left(P \rightarrow X, \nabla_{P}\right)$ be the associated $\mathbb{P}^{1}$-bundle. Now since the map "multiplication by -1 " on an elliptic curve induces the map "multiplication by -1 " on $\mathscr{E}$, it follows that $\left(\mathscr{E}, \nabla_{\mathscr{E}}\right)$ will not descend via $\Lambda$. However, since "multiplication by -1 " induces the identity on $P \rightarrow X$, we see that $\left(P \rightarrow X, \nabla_{P}\right)$ does descend via $\Lambda$. This gives us an in digenous bundle on $X^{\text {log }}$. To see that it is nilpotent and admissible, it suffices to see that $\left(\mathscr{E}, \nabla_{\mathscr{E}}\right)$ is nilpotent and admissible. But by (a rather trivial special case of ) [Falt], Theorem 6.2, as a de Rham cohomology module, $\left(\mathscr{E}, \nabla_{\mathscr{E}}\right.$ ) necessarily forms an $\mathcal{M} \mathscr{F}^{\nabla}$-object (see Definition 2.9). Thus, Proposition 2.10 tells us that $\left(\mathscr{E}, \nabla_{\mathscr{E}}\right)$ is nilpotent and admissible. In particular. this completes the proof of Corollary 2.15 .

Now let us assume that $f^{\log }: X^{\log } \rightarrow S^{\text {log }}$ is formed by gluing together a number of copies of the 3-pointed stable curve of genus zero (as in the last subsection of Chapter I, §2). Then, as we saw in this final subsection of Chapter I, $\S 2$, we can glue together the nilpotent, admissible indigenous bundles that we constructed in the previous paragraph to obtain a nilpotent. admissible indigenous bundle $\left(P \rightarrow X, \nabla_{P}\right)$ on $X^{\mathrm{log}}$. On the other hand, by Proposition 2.8, every nilpotent, admissible indigenous bundle on $X^{\log }$ is of restrictable type. Since there is (up to isomorphism) only one indigenous $\mathbb{P}^{1}$-bundle of restrictable type on $X^{\log }$. it thus follows that:

Proposition 3.5. Up to isomorphism, a totally degenerate r-pointed stable curve of genus $g$ admits one and only one nilpotent. admissible indigenous $\mathbb{P}^{1}$-bundle.

Next, let us consider the cohomology group $H^{1}\left(X, \tau_{X^{108} / S^{108}}\right)$ of our totally degenerate curve. If $X^{\mathrm{log}}$ is obtained by gluing together various copies $X_{i}^{\log }$ of the 3 -pointed stable curve of genus zero, let $Y^{\text {log }}$ be the disjoint union of the $X_{1}^{\text {log }}$, and let $\nu^{\log :} Y^{\log } \rightarrow X^{\log }$ be the natural map. Let $0 \rightarrow \tau_{X^{108} / S^{108}} \rightarrow \nu^{*} \tau_{Y^{108} / s^{108}} \rightarrow \mathscr{C} \rightarrow 0$ be the natural exact sequence (where $\mathscr{C}$ is defined so as to make the sequence exact). By considering the long exact cohomology sequence associated to this exact sequence of sheaves, we see that we obtain a natural isomorphism $H^{1}(X$, $\tau_{\left.X^{108} / S^{108}\right) \cong H^{0}}(X, \mathscr{C})$. On the other hand, $\mathscr{C}$ is naturally isomorphic to the direct sum of the $\left(\left.\tau_{\left.\lambda^{108} / S^{108}\right)}\right|_{z}\right.$, where $z$ ranges over all the nodes in $X$. Moreover, the residue map gives a natural isomorphism $\left.\tau_{\lambda^{108} / S^{108}}\right|_{z} \cong \mathscr{O}_{S}$ (well-defined $u p$ to sign). Let $\sum$ be the set of nodes of $X$. Then

$$
\mathscr{C}=\bigoplus_{z \in \Sigma}\left(\mathfrak{O}_{S}\right)_{z}
$$

where the subscript " $z$ " is just used as a marker, to indicate which copy of $\mathscr{O}_{S}$ 
one is referring to. Thus, we have a natural isomorphism (well-defined up to sign on each factor):

$$
H^{1}\left(X, \tau_{\lambda^{108} / S^{108}}\right) \cong \bigoplus_{z \in \Sigma}\left(\mathbf{F}_{p}\right)_{z}
$$

In particular, since $\mathbf{F}_{p}$ has a natural bilinear form (given by ring multiplication $\mathbb{F}_{p} \times \mathbb{F}_{p} \rightarrow \mathbf{F}_{p}$ ), using this bilinear form on each factor $\left(\mathbf{F}_{p}\right)_{z}$ gives a natural bilinear form:

$$
\mathscr{B}: H^{1}\left(X, \tau_{Y^{108} / S^{108}}\right) \times H^{1}\left(X, \tau_{X^{108} / S^{108}}\right) \rightarrow \mathbb{F}_{p}
$$

which is now independent of all arbitrary choices of sign.

Proposition 3.6. For every totally degenerate r-pointed curve of genus $g$. there is a natural nondegenerate bilinear form $\mathscr{B}$ on the $\mathscr{O}_{S}$-module $\mathbb{R}^{1} f_{*} \tau_{\mathrm{I}^{108} / S^{\text {1.8 }}}$ which takes values in $\mathscr{O}_{S}$.

Next we would like to show that the unique nilpotent, admissible indigenous bundle $\left(P \rightarrow X, \nabla_{P}\right)$ on $X^{\log }$ is ordinary. To do this. we must compute the induced Frobenius action on $H^{1}\left(X, \tau_{Y^{\log _{8} / S^{108}}}\right)$. By using an isomorphism as above

$$
H^{1}\left(X, \tau_{\left.1^{100 / S^{102}}\right)} \cong \bigoplus_{z \in \Sigma}\left(\mathbb{F}_{p}\right)_{z}\right.
$$

we see that it suffices to compute the induced Frobenius action on the various $\left(\mathbb{F}_{p}\right)_{z}$ 's. Consider $\left.P\right|_{z}$. The Hodge section $\sigma: X \rightarrow P$ defines a point $\left.\sigma_{z} \in P\right|_{z}\left(\mathbf{F}_{p}\right)$. On the other hand, there is a unique point fixed by the monodromy action $q_{z} \in$ $\left.P\right|_{z}\left(\mathbb{F}_{p}\right)$. If we think of $P$ as $\mathbf{P}\left(\mathscr{g} / \mathscr{g}^{[3]}\right)$ (as in Chapter I, Proposition 2.5), then $\left(\mathscr{g} / \mathscr{g}^{[3]}\right)_{z}=V_{\sigma} \oplus V_{q}$, where $V_{\sigma}$ (respectively, $V_{q}$ ) is the subspace defined by $\sigma_{z}$ (respectively, $q_{z}$ ). Note that by the residue map. we have natural isomorphisms $V_{\sigma} \cong \mathbb{F}_{p}$ and $V_{q} \cong \mathbb{F}_{p}$. Thus, we obtain a basis $\{(1,0) ;(0,1)\}$ of $\left(\mathscr{g} / \mathscr{g}^{[3]}\right)_{z}$. Let $E_{\sigma}$ and $E_{q}$ be the nilpotent endomorphisms of $\left(\mathscr{g} / \mathscr{g}^{[3]}\right)_{z}$ given, respectively, by the matrices

$$
\left(\begin{array}{ll}
0 & 1 \\
0 & 0
\end{array}\right) \text { and }\left(\begin{array}{ll}
0 & 0 \\
1 & 0
\end{array}\right)
$$

Thus, $E_{q}$ is essentially the $p$-curvature of $\left(\pi: P \rightarrow X, \nabla_{P}\right)$ restricted to $z$. Sorting through all the definitions, it thus follows that the induced Frobenius action on $\left(\mathbb{F}_{p}\right)_{z}$ is given by multiplication by $\operatorname{tr}\left(E_{\sigma} \cdot E_{q}\right)=1$. We thus obtain the following result :

Proposition 3.7. On a totally degenerate r-pointed stable curve $X^{\log }$ of genus $g$ over $\mathbf{F}_{p}$, the Frobenius action $\Phi_{P}^{\tau}$ on $H^{1}\left(X, \tau_{X^{\mathrm{vog}} / S^{\mathrm{log}}}\right)$ induced by the unique nilpo- 
tent, admissible indigenous bundle on $X^{\log }$ is the identity. In particular, this unique nilpotent, admissible indigenous bundle is ordinary, and so is $X^{\mathrm{log}}$.

Corollary 3.8. The open subschemes $\bar{M}_{g, r}^{\text {ord }} \subseteq \bar{M}_{g, r}$ and $\overline{\mathcal{N}}_{g, r}^{\text {ord }} \subseteq \overline{\mathcal{N}}_{g, r}$ are nomempty.

\section{The Case of Elliptic Curves: The Parabolic Picture}

One can get a better feel for ordinariness for general $r$-pointed stable curves of genus $g$ by first studying ordinariness for elliptic curves. In the case of elliptic curves, there are, in fact. two possible theories of ordinary bundles and curves: the parabolic theory and the hyperbolic theory. Indeed, let $f^{\text {log }}$ : $X^{\log } \rightarrow S^{\log }$ be a 1 -pointed stable curve of genus 1 , with marked point $\epsilon: S \rightarrow X$. Let $Y^{\log }$ be the log scheme obtained from $X^{\log }$ (as in the subsection "The Infinitesimal Verschiebung" of $\$ 2$ ) by removing the marked point. Then the parabolic theory (respectively, hyperbolic theory) is obtained by considering the various properties of the $p$-curvature of indigenous bundles on $Y^{\log }$ (respectively, $\left.X^{\mathrm{log}}\right)$. So far in this Chapter, of course, we have only been considering the hyperbolic theory. However, since the notion of an indigenous bundle is defined for $Y^{\mathrm{log}}$, one can consider its $p$-curvature, and define the notions of a nilpotent indigenous bundle, or an admissible indigenous bundle, just as before. Also, many of the results (though not all) such as Theorem 2.3 (where we replace the " $3 g-3+r$ " by 1) continue to hold in the parabolic context. The purpose of this subsection is to summarize what happens when one studies elliptic curves from the parabolic point of view, and to show, in particular, that the notion of ordinariness that we have defined in this paper (in terms of the $p$-curvature of indigenous bundles) reduces to the classical notion of ordinariness of elliptic curves.

First, we introduce some notation. Let $\bar{M}_{1,0}^{\text {log }}=\bar{M}_{1,1}^{\text {log }}$. (The point here is that we shall use the notation $\bar{M}_{1,0}^{\text {log }}$ when we are thinking about elliptic curves from the parabolic point of view.) Let $f: \mathscr{G} \rightarrow \bar{M}_{1,0}$ be the universal elliptic curve, with identity section $\epsilon: \bar{M}_{1,0} \rightarrow \mathscr{G}$. Let $\mathscr{L}=f_{*} \omega \mathscr{G} / \bar{M}_{10}$ be the Hodge bundle. Let $\mathscr{G}^{\log }$ be the log stack whose underlying stack is $\mathscr{G}$ and whose log structure is defined by the pull-back of the divisor at infinity of $\bar{M}_{1,0}$. Let $\bar{\varnothing}_{1,0} \rightarrow \bar{M}_{1,0}$ be the torsor over $\mathscr{L}^{\otimes 2}$ of Schwarz structures on $\mathscr{G}^{\log }$. Then just as before, we can define a Verschiebung:

$$
\bar{V}_{1,0}: \bar{ষ}_{1,0} \rightarrow \overline{\mathscr{Q}}_{1,0}
$$

Just as before we have a closed subscheme $\overline{\mathcal{N}}_{1,0} \subseteq \bar{\varnothing}_{1,0}$ consisting of nilpotent in- 
digenous bundles, and an open subscheme $\overline{\mathscr{X}}_{1,0}^{\text {adm }} \subseteq \overline{\mathscr{X}}_{1,0}$ consisting of admissible indigenous bundles. Also, just as before, we define an indigenous bundle to be ordinary if its infinitesimal Verschiebung is an isomorphism, and we define an elliptic curve to be (parabolically) ordinary if it admits a nilpotent, ordinary indigenous bundle.

Now recall that in Example 2 of Chapter I, §2, we constructed a canonical indigenous bundle on $g^{10 g}$. This indigenous bundle thus defines a global section $\tau_{s}: \bar{M}_{1,0} \rightarrow \bar{\Phi}_{1,0}$ which trivializes the $\mathscr{L}^{\otimes 2}$-torsor $\bar{\Phi}_{1,0} \rightarrow \bar{M}_{1,0}$. Moreover, by $[\mathrm{KM}], \mathrm{p} .227$, one knows that if $p \geq 5$, then $H^{0}\left(\bar{M}_{1,0}, \mathscr{L}^{\otimes 2}\right)=0$, so this trivialization is unique. Let us also recall that, in the definition of the indigenous bundle $\left(\mathscr{E} . \nabla_{\mathscr{E}}\right)$ in Example 2 of Chapter I, $\S 2$, we had a subbundle $0 \oplus \mathscr{O}_{\xi} \subseteq \omega \oplus \mathscr{O}_{\mathscr{G}}=\mathscr{E}$ (where $\omega=\omega \mathscr{G} / \bar{M}_{10}$ ) which was stabilized by the connection $\nabla_{\mathscr{E}}$. Moreover, the induced connection on $\mathscr{O}_{\mathscr{G}}$ was the trivial connection. Put another way, $\mathscr{E}$ admits a nonzero horizontal section. It thus follows that the $p$-curvature of $\nabla_{\mathscr{E}}$ is nilpotent. Thus, $\tau_{\&}: \bar{M}_{1,0} \rightarrow \bar{\bigotimes}_{1,0}$ lands inside $\overline{\mathcal{N}}_{1,0}$.

Let $\theta$ be a section of $\mathscr{L}^{\otimes 2}$ over some étale $V \rightarrow \bar{M}_{1,0}$. Let $\nabla_{\mathscr{8}}^{\theta}$ be the connection formed by adding to $\nabla_{\Xi}$ the endomorphism given by

$$
\mathscr{E}=\omega \oplus \mathscr{O}_{\mathscr{G}} \rightarrow \mathscr{O}_{\varphi} \rightarrow \omega^{\otimes 2} \cong(\omega \oplus 0) \otimes \omega \subseteq \mathscr{E} \otimes \omega
$$

where the first morphism is the natural projection; the second morphism is multiplication by $\theta$; and the final inclusion is the natural one. Let $\delta$ be a section of $\mathscr{L}$ over $V$ which is everywhere nonzero. Thus, the sections $\delta$ and 1 define a global trivialization of $\mathscr{E}=\omega \oplus \mathscr{O}_{\mathscr{G}}$ over $\mathscr{G}_{V}$. We shall write sections of $\left.\mathscr{E}\right|_{V}$ in terms of this basis, given by $\delta$ and 1. Write $\theta=\phi \cdot \delta^{2}$, where $\phi$ is a function on $V$. Let $\nabla^{\theta}$ be the morphism $\left.\left.\mathscr{E}\right|_{V} \rightarrow \mathscr{E}\right|_{V}$ given by evaluating $\nabla_{\mathscr{8}}^{\theta}$ on $\delta^{-1}$. Then we see that $\nabla^{\theta}$ is given by the following matrix :

$$
\left(\begin{array}{ll}
0 & \phi \\
1 & 0
\end{array}\right)
$$

To obtain the $p$-curvature of $\nabla_{\tilde{b}}^{\theta}$, we must iterate this matrix $p$ times. This yields the matrix :

$$
\phi^{\frac{1}{2}(p-1)} \cdot\left(\begin{array}{ll}
0 & \phi \\
1 & 0
\end{array}\right)
$$

Now let us write $\delta^{-p}=h \cdot \delta^{-1}$ (so $h$ is the classical Hasse invariant). Thus, to compute the $p$-curvature of $\nabla_{\mathscr{\delta}}^{\theta}$, we must subtract from the matrix just given the following matrix: 


$$
h \cdot\left(\begin{array}{ll}
0 & \phi \\
1 & 0
\end{array}\right)
$$

Thus, we obtain that the $p$-curvature of $\nabla_{\mathscr{E}}^{\theta}$ is given by:

$$
\left(\phi^{\frac{1}{2}(p-1)}-h\right) \cdot\left(\begin{array}{ll}
0 & \phi \\
1 & 0
\end{array}\right)
$$

If we then take the determinant, we obtain our Verschiebung (applied to $\left(\delta^{F}\right)^{2}$ ):

$$
-\phi \cdot\left(\phi^{\frac{1}{2}(p-1)}-h\right)^{2}=-\phi^{p}+2 h \cdot \phi^{\frac{1}{2}(p+1)}-h^{2} \cdot \phi
$$

Let us rewrite this in invariant form. The trivialization $\tau_{\xi}$ of $\bar{\Phi}_{1,0} \rightarrow \bar{M}_{1,0}$ allows us to write $\overline{\mathscr{X}}_{1,0}$ as $\operatorname{Spec}\left(\oplus_{i \geq 0} \mathscr{L}^{\otimes-2 i}\right)$. On the other hand, $\overline{\mathscr{Q}}_{1,0}$ is given by $\operatorname{Spec}\left(\oplus_{1 \geq 0} \mathscr{L}^{\otimes-2 p \cdot \imath}\right)$. Thus, $\overline{\mathscr{V}}_{1,0}$ is determined by specifying the morphism of quasi-coherent sheaves :

$$
\Gamma_{\mathscr{V}}: \mathscr{L}^{\otimes-2 p} \rightarrow \underset{1 \geq 0}{\bigoplus} \mathscr{L}^{\otimes-2 \imath}
$$

Let us denote the component of $\Gamma_{v}$ that maps into $\mathscr{L}^{\otimes-2 \imath}$ by $\Gamma_{\psi}^{[\imath]}: \mathscr{L}^{\otimes-2 p} \rightarrow \mathscr{L}^{\otimes-2 \imath}$. Since $\mathscr{L}$ is ample, it follows that $\Gamma_{V}^{[2]}=0$ when $i>p$ (as we saw already in the proof of Theorem 2.3). Let $\chi \in \Gamma\left(\bar{M}_{1,0}, \mathscr{L}^{p-1}\right)$ be the Hasse invariant (as in $[\mathrm{KM}], \mathrm{p} .353)$. Then we see that we have proven the following result:

Theorem 3.9. If $i=p$, then $\Gamma_{V}^{[1]}$ is multiplication by -1 . If $i=\frac{1}{2}(p+1)$, then $\Gamma_{\psi^{[i]}}^{[s}$ is multiplication by $2 \chi$. If $i=1$, then $\Gamma_{v}^{[i]}$ is multiplication by $-\chi^{2}$. For all other $i, \Gamma_{ף}^{[2]}=0$.

In particular, this completes the proof of Theoren 2.3 .

Corollary 3.10. Geometrically, $\overline{\mathcal{N}}_{1,0}$ consists of two irreducible components $\mathscr{I}_{1}$ and $\mathscr{I}_{2}$ : One, $\mathscr{I}_{1}$, is the section $\tau_{\searrow}$. The other, $\mathscr{I}_{2}$, is nonreduced, and $\left(\mathscr{I}_{2}\right)_{\text {red }}$ may be described as follows: In $[K M], p .361$, one finds a description of the Igusa curve $\operatorname{Ig}(p)$. with its canonical $(\mathbb{Z} / p \mathbb{Z})^{\times}$-action. Then $\left(\mathscr{I}_{2}\right)_{\text {red }}$ is the quotient of $\operatorname{Ig}(p)$ by the subgroup $\{ \pm 1\} \subseteq(\mathbb{Z} / p \mathbb{Z})^{\times}$.

In order to see which nilpotent bundles are ordinary, we must compute the derivative of the Verschiebung map. In terms of the local objects we used in the computation above, we obtain that " $d \mathscr{V} / d \phi$ " is given by:

$$
h\left(\phi^{\frac{1}{2}(p-1)}-h\right)
$$


In particular, if $h=0$, then the infinitesimal Verschiebung is identically zero, while if $h \neq 0$, then the infinitesimal Verschiebung at $\tau_{\&}$ is nonzero. Moreover. because of the square factor in the expression for the Verschiebung, we see that if $h \neq 0$, then the only nilpotent indigenous bundle at which the infinitesimal Verschiebung will be nonzero is the indigenous bundle given by $\tau_{\&}$. We thus obtain the following:

Theorem 3.11. An elliptic curve is parabolically ordinary if and only if it is ordinary in the classical sense (i.e., its Hasse invariant is nonzero). If it is ordinary, then the indigenous bundle constructed in Example 2 of Chapter I, $\S 2$, is the unique nilpotent, ordinary indigenous bundle on the curve.

\section{The Case of Elliptic Curves: The Hyperbolic Picture}

In this subsection, we consider 1-pointed stable curves of genus 1 as hyperbolic objects. In particular, we shall highlight the numerous contrasts with the parabolic viewpoint presented above. We begin by considering the torsor $\bar{\bigotimes}_{1,1}$ $\rightarrow \bar{M}_{1,1}$. Recall that this torsor has a canonical trivialization at infinity, defined by the unique nilpotent, admissible indigenous bundle on the singular 1-pointed stable curve of genus 1 (Proposition 3.5). Let us suppose that our prime $p$ is $\geq 5$. Then it follows from Chapter I, Theorem 3.6, that $\overline{\mathscr{S}}_{1,1} \rightarrow \bar{M}_{1,1}$ does not admit a section which passes through the canonical trivialization at infinity. Now let us consider the closed subscheme $\overline{\mathcal{N}}_{1,1} \subseteq \overline{\mathscr{\Phi}}_{1,1}$. By Theorem 2.3, the natural morphism $\overline{\mathcal{N}}_{1,1} \rightarrow \bar{M}_{1,1}$ is finite and flat of degree $p$. Let us consider the irreducible component $\mathscr{I} \subseteq \overline{\mathcal{N}}_{1,1}$ which passes through the canonical trivialization at infinity. Then $\mathscr{I}$ is generically reduced. Moreover, the degree of $\mathscr{I} \rightarrow \bar{M}_{1,1}$ must be $\geq 2$. This behavior already is substantially different from the parabolic case, where the irreducible component passing through the unique nilpotent, ordinary indigenous bundle at infinity has degree one over $\bar{M}_{1,0}$. Thus, in particular, $\overline{\mathcal{N}}_{1,1}$ (respectively, $\overline{\mathscr{V}}_{1,1}$ ) is not isomorphic to $\overline{\mathcal{N}}_{1,0}$ (respectively, $\overline{\mathscr{V}}_{1,0}$ ), despite the fact that as stacks. $\bar{M}_{1,1} \cong \bar{M}_{1,0} ; \overline{\mathscr{ष}}_{1,1} \cong \overline{\mathscr{Q}}_{1,0} ; \overline{\mathscr{Q}}_{1,1} \cong \overline{\mathscr{Q}}_{1,0}$. Since $\overline{\mathcal{N}}_{1,1} \rightarrow$ $\bar{M}_{1,1}$ has degree $p$, it follows that there exist points of $\overline{\mathcal{N}}_{1,1}$ over the infinity point of $\bar{M}_{1,1}$ at which $\overline{\mathcal{N}}_{1,1} \rightarrow \bar{M}_{1,1}$ is not étale. Such points correspond to nilpotent indigenous bundles which are not admissible (by Proposition 3.5). This fulfills our earlier pledge to show the existence of such bundles. Of these various observations, we record the following for later reference:

Proposition 3.12. If $p \geq 5$, then the irreducible component of $\overline{\mathcal{N}}_{1,1}$ passing through the canonical trivialization at infinity is generically reduced and has 
degree $\geq 2$ over $\bar{M}_{1,1}$

\section{The Generic Uniformization Number}

We return to the case of an $r$-pointed stable curve of genus $g$, where $r$ and $g$ are arbitrary (but satisfy $2 g-2+r \geq 1$ ). Suppose we are given the combinatorial data $\Delta$ (as at the end of Chapter I. Section 2 : consisting of a graph $\Gamma$, plus $\lambda_{i}$ 's, etc.) for a totally degenerate curve. We shall call two collections of such data $\Delta$ and $\Delta^{\prime}$ equivalent if they define isomorphic totally degenerate curves. Let us denote by $\mathscr{D}_{g . r}$ the equivalence classes of such data $\Delta$. Alternatively, one may think of $\mathscr{D}_{g, r}$ as the set of isomorphism classes of totally degenerate $r$-pointed stable curves of genus $g$. Now let us consider the morphism:

$$
\overline{\mathcal{N}}_{g, r} \rightarrow \bar{M}_{g, r}
$$

We know that it is finite, flat, and of degree $p^{3 g-3+r}$. If $\Delta \in \mathscr{D}_{g, r}$, consider the irreducible component $\mathscr{I}_{\Delta}$ of $\overline{\mathcal{N}}_{g, r}$ that passes through the unique nilpotent. admissible indigenous bundle (as in Proposition 3.5) on the curve corresponding to $\Delta$. Then $\mathscr{I}_{\Delta}$ is generically reduced. Let $G_{\Delta}$ be the degree of $\mathscr{I}_{\Delta}$ over $\bar{M}_{g, r}$. We shall refer to $G_{\Delta}$ as the generic unifornization number for the data $\Delta$. (The reason for attaching the term "uniformization" to this number will become apparent in later Chapters.) Let

$$
G_{g, r}=\sum_{\Delta \in \mathscr{D}_{0},} G_{\Delta}
$$

Now let us suppose that $p$ is sufficiently large so that the class $\sum$ (Chapter I, Theorem 3.4) in $H^{1}\left(\bar{M}_{q, r}, \Omega_{\bar{M}_{g r}^{\text {log }}}\right)$ is nonzero. Then we have the following rough result :

Proposition 3.13. For $g \geq 3$ and $p$ sufficiently large, the number $G_{\Delta}$ is between 2 and $p^{3 g-3+r}$.

Proof. The upper bound follows from the fact that $\overline{\mathcal{N}}_{g, r} \rightarrow \bar{M}_{g, r}$ has degree $p^{3 g-3+r}$. On the other hand, since $\overline{\mathcal{N}}_{g, r} \rightarrow \bar{M}_{g, r}$ is finite, and $\bar{M}_{g, r}$ is normal. it follows that if $\mathscr{I}_{\Delta}$ had degree 1 over $\bar{M}_{g, r}$, it would, in fact, be isomorphic to $\bar{M}_{g, r}$, hence define a section of $\overline{\mathscr{\phi}}_{g, r}$ over $\bar{M}_{g, r}$. By Chapter I. Theorem 3.4, we know that this is impossible, for $p$ sufficiently large.

It is not clear to the author how far these bounds are from being sharp. For instance, it could be the case that $\overline{\mathcal{N}}_{g, r}$ is, in fact, irreducible. To compute the number $G_{\Delta}$ exactly would involve understanding the monodromy around 
curves that are not hyperbolically ordinary. That is to say, it would involve proving a sort of hyperbolic analogue of Igusa's theorem on the monodromy around supersingular elliptic curves in the parabolic case.

It is interesting to know, however, that $G_{\Delta} \neq 1$ because this constitutes a departure from the behavior of complex indigenous bundles. To see this, we must first explain certain aspects of the analogy between the complex case and the characteristic $p$ case treated here. First of all, the condition of being nilpotent (and ordinary) is analogous, in the complex case, to having real monodromy. Indeed, to be nilpotent (and admissible) is (by Proposition 2.10) the same as coming from an $\mathcal{M} \mathscr{F}^{\nabla}$-object in the sense of [Falt]. But to be an $\mathcal{M F}^{\nabla}$-object means, essentially, that the bundle with connection admits a Frobenius action, i. e., that the monodromy is Frobenius-invariant. Since the Frobenius at the in finite prime is complex conjugation, it is thus natural to regard nilpotent (and admissible) bundles as the characteristic $p$ analogue of complex indigenous bundles with real monodromy. On the other hand, in the complex case, within the real-analytic space of complex indigenous bundles with real monodromy, there is a canonical, topologically isolated component, corresponding to the indigenous bundle arising from the uniformization by the upper half-plane. On the other hand, in the characteristic $p$ case treated here, the fact that $\mathscr{I}_{\Delta}$ has degree $\geq 2$ over $\bar{M}_{g, r}$ means that there is no canonical choice of a nilpotent, ordinary indigenous bundle, even on a generic curve: since the monodromy at the curves which are not hyperbolically ordinary is nontrivial, one such indigenous bundle is always carried around to another.

\section{Chapter III: Canonical Modular Frobenius Liftings}

\section{§. Introduction}

The present Chapter is central to the entire paper. In it, we construct a canonical Frobenius lifting on $\left(\overline{\mathcal{N}}_{g, r}^{\text {ord }}\right)^{\text {log }}$, and a canonical indigenous bundle on the the universal curve over $\left(\overline{\mathcal{N}}_{g, r}^{\text {ord }}\right)^{\text {log }}$. This pair of a canonical Frobenius lifting and a canonical indigenous bundle are uniquely characterized by the fact that, relative to this Frobenius lifting, the renormalized Frobenius pull-back (Definition 1.4) of the canonical indigenous bundle is equal to itself. In some sense, an ordinary (in the sense of Definition 1.1) Frobenius lifting is like a $p$-adic ana- 
logue of a Kähler metric on a complex manifold in that it gives rise to local canonical coordinates. Since there are a number of general properties of ordinary Frobenius liftings that we will need throughout the rest of the paper, we give a basic exposition of the properties of such Frobenius liftings in the first Section of this Chapter. The main result is that such a Frobenius lifting defines canonical affine and multiplicative coordinates. Thus, in particular, our canonical Frobenius lifting on $\left(\overline{\mathcal{N}}_{g, r}^{\text {ord }}\right)^{109}$ defines such canonical coordinates on $\overline{\mathcal{N}}_{g, r}^{\text {ord }}$. Since $\overline{\mathcal{N}}_{g, r}^{\text {ord }}$ is étale over $\bar{M}_{g, r}$, if one thinks of a point of $\overline{\mathcal{N}}_{g, r}^{\text {ord }}$ as a point of $\bar{M}_{g . r}$, together with a choice (from a finite number of possibilities) of some added structure - which we call a p-adic quasiconformal equivalence class - then we obtain the result that for every choice of a $p$-adic quasiconformal equivalence class, we obtain a canonical local uniformization of $\mathcal{M}_{g, r}$. The reason for the name "quasiconformal equivalence class," is that once one chooses this piece of data for a curve, we shall see in this Chapter and in following Chapters that the uniformization theory of the curve is entirely determined. This is reminiscent of Bers' approach (as in [Bers]) to proving that hyperbolic curves can be uniformized by the upper half plane: Namely, he proves that (in the complex case) all hyperbolic curves with the same genus and number of marked points belong to the same quasiconformal equivalence class. Thus, once we choose this class in the $p$-adic case, we obtain a "covariant" uniformization by the affine space modeled on the tangent space to $\mathcal{M}_{g, r}$ at the curve in question. To obtain uniformizations by the quadratic differentials (as in the complex case), we need more information than just the quasiconformal equivalence class. Namely, we need a topological marking of the curve. Once we define this, we obtain uniformizations by the quadratic differentials.

The canonical Frobenius gives rise to another natural notion, for which I know no parallel in the complex case: a canonical lifting of a curve over a perfect field to the ring of Witt vectors with coefficients in that field. This is reminiscent of the canonical lifting of an elliptic curve in Serre-Tate theory. In fact, this analogy is more than philosophical: Just as in Chapter II, by using in digenous bundles on elliptic curves - regarded parabolically - one can obtain a similar uniformization theory, involving a canonical Frobenius lifting (on the moduli stack of ordinary elliptic curves) and a canonical indigenous bundle. We then compute that these canonical objects for elliptic curves are precisely the canonical objects that one obtains from classical Serre-Tate theory. Thus, one may regard the theory of uniformizations and canonical liftings discussed in this Chapter as the natural hyperbolic analogue of Serre-Tate theory. 


\section{§1. Generalities on Ordinary Frobenius Liftings}

Let $k$ be a perfect field of odd characteristic $p$. Let $A=W(k)$, the ring of Witt vectors with coefficients in $k$. Let $S$ be a formally smooth, geometrically connected $p$-adic formal scheme over $A$ of constant relative dimension $d$. Let $S^{\log }$ be a log formal scheme whose underlying formal scheme is $S$ and whose log structure is given by a relative divisor with normal crossings $D \subseteq S$ over $A$. Let $\Phi_{A}: A \rightarrow A$ be the Frobenius morphism on $A$. Let us denote the result of base changing by $\Phi_{A}$ by means of a superscripted " $F$." Let $\Phi^{\log }: S^{\log } \rightarrow S^{\text {log }}$ be a Frobenius lifting, i.e., a morphism whose reduction modulo $p$ is the usual Frobenius morphism in characteristic $p$. In this Section, we shall study the case of a certain kind of Frobenius lifting, called an ordinary Frobenius lifting. It turns out that such Frobenius liftings define various types of canonical parameters. It is these canonical parameters that will constitute the various "uniformizations" that we obtain in this paper.

\section{Basic Definitions}

Let us consider the morphism

$$
d \Phi^{\log }: \Phi^{*} \Omega_{S / A}^{\log _{A}} \rightarrow \Omega_{S / A}^{\log }
$$

induced by $\Phi^{\text {log }}$ on logarithmic differentials. It is always divisible by $p$.

Definition 1.1. We shall call $\Phi^{\log }: S^{\log } \rightarrow S^{\log }$ an ordinary Frobenius lifting if $\frac{1}{p} \cdot d \Phi^{\log }: \Phi^{*} \Omega_{S / A}^{\log _{A}} \rightarrow \Omega_{S / A}^{\log }$ is an isomorphism.

We shall use the notation

$$
\Omega_{\Phi}: \Phi^{*} \Omega_{S / A}^{\log _{A}} \rightarrow \Omega_{S / A}^{\log _{A}}
$$

to denote the isomorphism $\frac{1}{p} \cdot d \Phi^{\log }$. Note that by considering the sections of $\Omega_{S / A}^{\log }$ which are invariant under $\Omega_{\Phi}$, we obtain an étale sheaf $\Omega_{\Phi}^{\text {et }}$ in free $\mathbb{Z}_{p}$-modules of rank $d$ on $S$.

Definition 1.2. We shall call $\Omega_{\Phi}^{\mathrm{et}}$ the canonical differential local system on $S$ associated to $\Phi^{109}$. We shall call its dual $\Theta_{\Phi}^{\mathrm{et}}$ the canonical tangential local sys- 
tem on $S$ associated to $\Phi^{\text {log }}$.

Moreover, by taking the sections of $\Omega_{\Phi}^{\text {et }}$ to be horizontal, we obtain a natural connection $\nabla_{\Omega}$ on $\Omega_{S / A}^{\log }$ which is associated to $\Phi^{100}$. Note that since $\Omega_{\Phi}^{\text {et }}$ is an étale (not just log étale) local system on $S$, the connection $\nabla_{\Omega}$ is a connection on $S$ with respect to the trivial log structure, i.e., it has no logarithmic poles at $D$.

\section{The Uniformizing Galois Representation}

Now we would like to associate to $\Phi^{\text {log }}$ a canonical "uniformizing $\mathcal{M} \mathscr{F}^{\nabla}$-object" (where we use the category $\mathcal{M} \mathscr{F}^{\nabla}$ in the sense of [Falt], §2) as follows. Let

$$
\mathscr{P}=\Omega_{S / A}^{\log } \oplus \mathcal{O}_{S}
$$

We regard $\mathscr{P}$ as being filtered by taking the filtration

$$
F^{2}(\mathscr{P}) \stackrel{\text { def }}{=} 0 ; F^{1}(\mathscr{P}) \stackrel{\text { def }}{=} \Omega_{S / A}^{\log _{A}} \oplus 0 \subseteq \mathscr{P} ; F^{0}(\mathscr{P}) \stackrel{\text { def }}{=} \mathscr{P}
$$

Let $\nabla_{\mathscr{p}}$ be the logarithmic connection on $\mathscr{P}$ obtained as follows: We start with the connection $\nabla_{\mathscr{P}}$ on $\mathscr{P}$ which is the direct sum of the trivial connection on $\mathscr{O}_{S}$ and the connection $\nabla_{\Omega}$ on $\Omega_{S / A}^{\log }$. Then we add to $\nabla_{\mathscr{P}}^{\prime}$ the End $(\mathscr{P})$-valued logarithmic differential given by

$$
\mathscr{P} \rightarrow \Omega_{S / A}^{\log } \cong\left(0 \oplus \mathscr{O}_{S}\right) \otimes_{\mathcal{U}_{S}} \Omega_{S / A}^{\log } \subseteq \mathscr{P} \otimes_{\mathcal{C}_{S}} \Omega_{S / A}^{\log _{A}}
$$

where the first morphism is the projection on the first direct summand. The resulting logarithmic connection on $\mathscr{P}$ will be called $\nabla_{\mathscr{P}}$. Note that the Kodaira-Spencer morphism for $F^{1}(\mathscr{P}) \subseteq \mathscr{P}$ with respect to $\nabla_{\mathscr{P}}$ is the identity map. Next, we define the Frobenius action on $\mathscr{P}$ as follows: We take the Frobenius action to be the morphism $\mathscr{P}_{\Phi}: \Phi * \mathscr{P} \rightarrow \mathscr{P}$ which is diagonal with respect to the direct sum decomposition $\mathscr{P}=\Omega_{S / A}^{\log } \oplus \mathscr{O}_{S}$ and is equal to $\Phi^{-1}$ on $\mathscr{O}_{S}$ and to $d \bar{\Phi}^{\log }$ on $\Omega_{S / A}^{\log }$. One sees easily that this Frobenius action $\mathscr{P}_{\Phi}$ is horizontal with respect to $\nabla_{\varphi p}$. Note that this implies that $\nabla_{\varphi p}$ is integrable, since its curvature would define a Frobenius-invariant section of $\left(\Omega_{S / A}^{\log _{A}}\right)^{\vee} \otimes_{\mathscr{O}_{S}} \wedge{ }^{2} \Omega_{S / A}^{\log _{A}}$, but $\Phi^{109}$ acts on this bundle with slope 1 , so any Frobenius-invariant section must necessarily vanish. Thus,

$$
\left(\mathscr{P}, F^{\cdot}(\mathscr{P}), \nabla_{\mathscr{P}}, \mathscr{P}_{\Phi}\right)
$$


defines an $\mathcal{M} \mathscr{F}^{\nabla}$-object in the sense of [Falt], §2.

Definition 1.3. We shall call this $\mathcal{M} \mathscr{F}^{\nabla}$-object the uniformizing $\mathcal{M F}^{\nabla}$-object on $S^{\log }$ associated to $\Phi^{\log }$.

Now choose a base point $s: \operatorname{Spf}(A) \rightarrow S$ that avoids $D$. Let $\Pi_{S^{100}}=\pi_{1}\left(S_{K}^{\text {log }}\right.$,

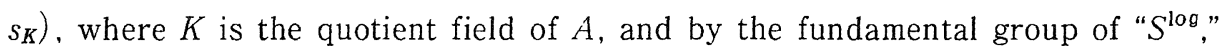
we mean the fundamental group of the open formal subscheme which is the complement of the divisors that define the log structure. Then by the theory of [Falt], §2, the uniformizing $M \mathscr{F}^{\nabla}$-object on $S^{\text {log }}$ defines a dual crystalline Galois representation

$$
\mathscr{P}_{\text {et }}
$$

of $\Pi_{\lrcorner 100}$.

Definition 1.4. We shall call this Galois representation the uniformizing Galois representation on $S^{109}$ associated to $\Phi^{109}$.

Note that $\mathscr{O}_{S} \cong\left(0 \oplus \mathscr{O}_{S}\right) \subseteq \mathscr{P}$ is stabilized by $\nabla_{\mathscr{P}}$ and $\mathscr{P}_{\Phi}$, and thus defines an $M \mathscr{F}^{\nabla}$-subobject of the uniformizing object which is equal to the trivial $M \mathscr{F}^{\nabla}$-object. If we take the quotient of the uniformizing $M \mathscr{F}^{\nabla}$-object by this subobject, we obtain the $\mathcal{M F}^{\nabla}$-object corresponding to the etale Galois representation $\Theta_{\Phi}^{\text {et }}$, Tate twisted once. Thus, we have an exact sequence of $\Pi_{S^{100}}$-modules

$$
0 \rightarrow \Theta_{\Phi}^{\mathrm{et}}(1) \rightarrow \mathscr{P}_{\mathrm{et}} \rightarrow \mathbb{Z}_{p} \rightarrow 0
$$

which thus defines an extension class:

$$
\eta_{\Phi} \in H^{1}\left(\Pi_{S^{109}}, \Theta_{\Phi}^{\mathrm{et}}(1)\right)
$$

We remark relative to the analogy between Frobenius liftings and Kähler metrics, that the class $\eta_{\Phi}$ formally "looks" somewhat like the differential form that defines a Kähler metric.

Also, we can define a ring with Galois action which will be useful later. First consider the symmetric algebra on $\Omega_{\Phi}^{\text {et }}(-1)$ over $\mathbb{Z}_{p}$ :

$$
\mathbf{S}_{\mathbf{Z}_{p}} \Omega_{\Phi}^{\mathrm{e}}(-1)
$$


Let us consider the Galois action of $\mathbb{I}_{S^{\text {ioe }}}$ on this symmetric algebra which differs from the direct sum of the actions on the $\mathbb{S}^{\imath}\left(\Omega_{\Phi}^{\text {et }}(-1)\right)$ by the class $\eta_{\Phi}$. Thus, in other words, Spec of the symmetric algebra with this Galois action parametrizes sections of the exact sequence

$$
0 \rightarrow \Theta_{\Phi}^{\mathrm{et}}(1) \rightarrow \mathscr{P}_{\mathrm{et}} \rightarrow \mathbb{Z}_{p} \rightarrow 0
$$

If we then adjoin the divided powers of the augmentation ideal to this $\mathbb{Z}_{p}\left[\Pi_{S^{100}}\right]$-algebra, we obtain a $\mathbb{Z}_{p}$-algebra $\mathscr{A l}_{\Phi}$. Let $T^{\log } \rightarrow S^{\log }$ be the finite

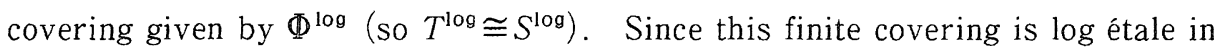
characteristic zero, we may form the subgroup $\Pi_{T^{100}} \subseteq \Pi_{S^{100}}$ corresponding to this

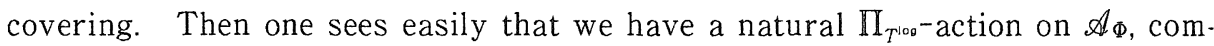
patible with the $\Pi_{S^{100}}$-action on the symmetric algebra. (We need to restrict to $\Pi_{T^{\text {IoO }}}$ so that the Galois action respects the divided powers.)

Definition $\mathbb{1}_{\text {.5. }}$. We shall call $\eta_{\Phi}$ the canonical Galois extension class associated to $\Phi^{\text {log. }}$. We shall call $\mathscr{A}_{\Phi}$ the ring of additive periods of $\Phi^{109}$.

\section{The Camonical p-divisible Group}

Let us look at the uniformizing $\mathcal{M} \mathscr{F}^{\nabla}$-object

$$
\left(\mathscr{P}, F(\mathscr{P}), \nabla_{\mathscr{P}}, \mathscr{P}_{\Phi}\right)
$$

again. Let $U \subseteq S$ be the open formal subscheme which is the complement of the divisor $U$. Then by $[\mathrm{Falt}]$, Theorem 7.1 , this $\mathcal{M} \mathscr{F}^{\nabla}$-object defines a $p$-divisible group $G_{\Phi}$ over $U$. Moreover, just as with the corresponding Galois representation discussed in the previous subsection. we have an exact sequence of $p$-divisible groups over $U$ :

$$
0 \rightarrow \Theta_{\Phi}^{\mathrm{et}}(1) \bigotimes_{\mathbf{Z}_{p}} \mathbb{Q}_{p} / \mathbb{Z}_{p} \rightarrow G_{\Phi} \rightarrow \mathbb{Q}_{p} / \mathbb{Z}_{p} \rightarrow 0
$$

Defirition 1.6. We shall call $G_{\Phi}$ the canonical $p$-divisible group associated to $\Phi^{\log }$.

Now let $\omega \in \Omega_{\Phi}^{\text {et }}$ be an element whose reduction modulo $p$ is nonzero. Thus, $\omega$ defines a $\mathbb{Z}_{p}$-linear morphism

$$
\omega(-): \Theta_{\Phi}^{\text {et }} \rightarrow \mathbb{Z}_{p}
$$


Let $S_{n} \rightarrow S$ be the étale covering defined by taking the kernel of the morphism $\Pi_{S^{100}} \rightarrow \mathrm{GL}\left(\Theta_{\Phi}^{\text {et }} \otimes \mathbf{Z} / p^{n} \mathbf{Z}\right)$. Let $U_{n} \rightarrow U$ be its restriction to $U$. Then over $U_{n}$, $\omega(-) \otimes \mathbf{Z} / p^{n} \mathbf{Z}$ will be Galois equivariant, so that, by pushing forward the above exact sequence by means of $\omega(-)$, we obtain an exact sequence of finite flat group schemes:

$$
0 \rightarrow \mathbb{Z} / p^{n} \mathbb{Z}(1) \rightarrow G_{\omega, n} \rightarrow \mathbb{Z} / p^{n} \mathbb{Z} \rightarrow 0
$$

which, by Kummer theory, defines an element

$$
u_{\omega, n} \in \Gamma\left(U_{n}, \mathscr{O}_{L_{n}}^{\times}\right) / \Gamma\left(U_{n}, \mathscr{O}_{L_{n}}^{\times}\right)^{p^{n}}
$$

and thus a differential

$$
\omega_{n}^{\prime} \stackrel{\text { def }}{=}\left(d \imath_{\omega, n}\right) / \iota_{\omega, n} \in \Gamma\left(U_{n}, \Omega_{l_{n} / d} \otimes \mathbb{Z} / p^{n} \mathbf{Z}\right)
$$

Now let $n \rightarrow \infty$. Let $\widetilde{S}$ be the $p$-adic completion of the inverse limit of the $S_{n}$. Since the various $\omega_{n}^{\prime}$ are compatible, we thus obtain a differential

$$
\omega^{\prime} \in \Gamma\left(\widetilde{U}, \Omega_{\widetilde{L} / A}\right)
$$

Now we would like to claim that $\omega^{\prime}$ is none other than the original differential $\omega$ that we started out with. In some sense, I believe that this fact is well-known, but I do not know of a clear reference for this fact, so I will prove it explicitly here. First, however, we need to make a few more general observations concerning $G_{\Phi}$. The proof will be given in the subsection after the next.

\section{Logarithms of Periods}

Suppose that $k$ is algebraically closed, and let $z: \operatorname{Spf}(A) \rightarrow S$ be a rational point whose reduction modulo $p$ is equal to the base point $s$. In particular, it follows that $z$ maps into $U$, and factors canonically through $\widetilde{U}$ (since it coincides with the base point $s$ modulo $p$ ). Thus, we can restrict the $G_{\omega, n}$ to $\operatorname{Spec}(A)$ via $z$ so as to obtain an extension

$$
0 \rightarrow \mathbb{Q}_{p} / \mathbb{Z}_{p}(1) \rightarrow G_{\omega, z} \rightarrow \mathbb{Q}_{p} / \mathbb{Z}_{p} \rightarrow 0
$$

of $p$-divisible groups over $\operatorname{Spec}(A)$. By Kummer theory, this extension defines a unit 


$$
u_{\omega, z} \in A^{\times}
$$

whose image in the residue field $k$ is 1 .

On the other hand, we can consider the Dieudonne crystal $E_{\omega}$ of $G_{\omega, z}$. Thus, $E_{\omega}$ is a free $A$-module of rank two with a filtration $F^{1}\left(E_{\omega}\right) \subseteq E_{\omega}$, and a Frobenius action $\Phi_{E}: E_{\omega}^{F} \rightarrow E_{\omega}$. This Frobenius action has a unique subspace $E_{F} \subseteq E_{\omega}$ (respectively, $E_{V} \subseteq E_{\omega}$ ) on which Frobenius acts with slope zero (respectively, one). Also, $E_{V}$ and $F^{1}\left(E_{\omega}\right)$ define the same subspace modulo $p$. Since $F^{1}\left(E_{\omega}\right)$ and $E / F^{1}\left(E_{\omega}\right)$ are naturally isomorphic to $A$, in the future, we shall identify them with $A$. Thus, by projection $E_{F} \hookrightarrow E_{\omega} \rightarrow E_{\omega} / F^{1}\left(E_{\omega}\right)=A$, we obtain a natural isomorphism of $E_{F}$ with $A$, and, dually, a natural isomorphism of $E_{V}$ with $A$. Finally, since $E_{\omega}=E_{F} \oplus E_{V}$, we may regard $F^{1}\left(E_{\omega}\right) \subseteq E_{\omega}$ as the graph of an $A$-linear morphism $A=E_{V} \rightarrow A=E_{F}$, which, by means of the various canonical trivializations, gives us an element $L_{\omega, z} \in p \cdot A$.

Theorem 1.7. We have $L_{\omega, z}=\log \left(u_{\omega, z}\right)$.

Proof. Let us denote the sequence of Galois modules which are the $p$-adic Tate modules of the above exact sequence of $p$-divisible groups by $0 \rightarrow W^{0} \rightarrow$ $W \rightarrow W^{-1} \rightarrow 0$. Recall the exponential map of [BK], p. 359, Definition 3.10.

$$
\exp : F^{1}\left(E_{\omega}\right)^{\otimes-2} \cong A \rightarrow A^{\times}=H_{\text {Gal }}^{1}\left(\mathbb{Z}_{p}(1)\right)
$$

where the first isomorphism is the trivialization referred to above, and $H_{\text {Gal }}^{1}$ denotes Galois cohomology with respect to $\mathrm{Gal}(\bar{K} / K)$, where $K$ is the quotient field of A. By [BK]. p. 359, Example 3.10.1, one knows that this exponential map is equal to the ordinary exponential map defined by the exponential series. Let $\eta_{2}=\log \left(u_{\omega, z}\right) ; \eta_{1}=\exp \left(\eta_{2}\right)$.

Now we diagram-chase. Let us denote by $\mathbb{P}$ the (infinite dimensional) Galois module $\mathbb{B}_{\text {crys }}^{\mathrm{f}=1} \oplus \mathbb{B}_{\mathrm{DR}}{ }^{+}$(notation of $[\mathrm{BK}]$ ). Applying the exact sequence $(1.17 .1)$ of $[\mathrm{BK}]$ to the exact sequence of Galois modules $0 \rightarrow W^{0} \rightarrow W$ $\rightarrow W^{1} \rightarrow 0$, we obtain the following commutative diagram: 


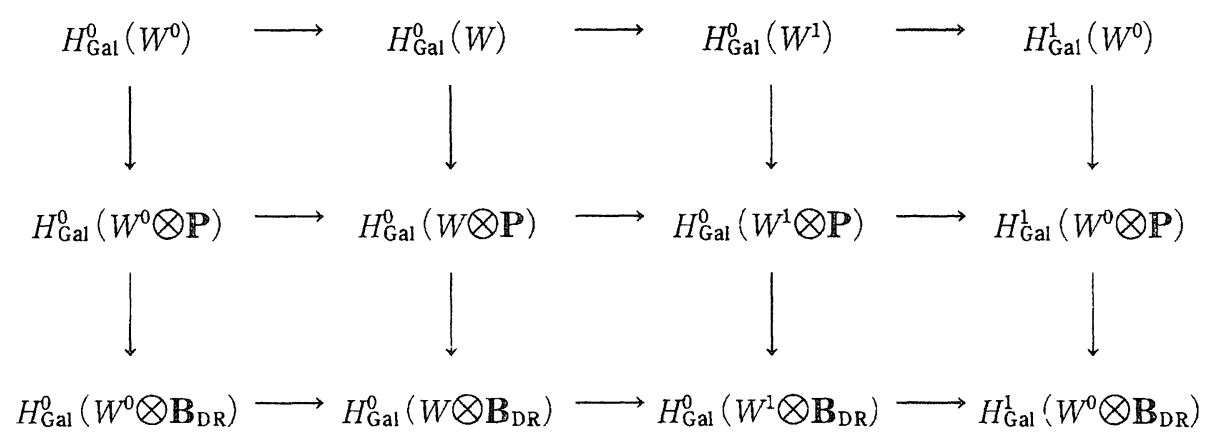

Now we have an element $1 \in H_{\text {Gal }}^{0}\left(W^{1}\right)$ which maps via the connecting homomorphism to $\eta_{1} \in H_{\mathrm{Gal}}^{1}\left(W^{0}\right)$; since the image of $\eta_{1}$ in $H_{\mathrm{Gal}}^{1}\left(W^{0} \otimes \mathbf{P}\right)$ is zero, we can consider $\log \left(\eta_{1}\right)=\eta_{2}$. On the other hand. $1 \in H_{\mathrm{Gal}}^{0}\left(W^{1}\right)$ maps to an element $\eta_{3} \in$ $H_{\mathrm{Gal}}^{0}\left(W^{1} \otimes \mathbb{P}\right)$ that dies when hit with the connecting homomorphism to $H_{\text {Gal }}^{1}\left(W^{0} \otimes \mathbb{P}\right)$. Thus we see that $\eta_{3}$ comes from an element $\eta_{4} \in H_{\text {Gal }}^{0}(W \otimes \mathbb{P})$ which is unique modulo $H_{\mathrm{Gal}}^{0}\left(W^{0} \otimes \mathbb{P}\right)$. Mapping $\eta_{4}$ down one step to $H_{\mathrm{Gal}}^{0}(W \otimes$ $\left.\mathbb{B}_{\mathrm{DR}}\right)$, we get $\eta_{5} \in H_{\mathrm{Gal}}^{0}\left(W \otimes \mathbb{B}_{\mathrm{DR}}\right)$ that dies in $H_{\mathrm{Gal}}^{0}\left(W^{1} \otimes \mathbf{B}_{\mathrm{DR}}\right)$, hence comes from a unique $\eta_{6} \in H_{\mathrm{Gal}}^{0}\left(W^{0} \otimes \mathbf{B}_{\mathrm{DR}}\right) / H_{\mathrm{Gal}}^{0}\left(W^{0} \otimes \mathbf{P}\right)=T V$. Now it follows from the explicit definitions of the maps in the sequence $(1.17 .1)$ of $[\mathrm{BK}]$ that $\eta_{6}$ is precisely $L_{\omega, z}$. On the other hand, it follows from general principles of homological algebra that $\eta_{6}=\eta_{2}$. This completes the proof.

\section{Compatibility of Differentials}

Now we return to the issue of showing that $\omega^{\prime}=\omega$. Let us begin by observing that $\omega$ can also be defined as follows. By taking the direct limit of the $G_{\omega, n}$ 's restricted to $\widetilde{U}$, we obtain an extension of $p$-divisible groups

$$
0 \rightarrow \mathbb{Q}_{p} / \mathbb{Z}_{p}(1) \rightarrow G_{\omega} \rightarrow \mathbb{Q}_{p} / \mathbb{Z}_{p} \rightarrow 0
$$

over $\widetilde{U}$. This, moreover, defines a Dieudonné crystal $\left(\mathscr{E}, \nabla_{\mathscr{E}}\right)$ with a filtration $F^{1}(\mathscr{E}) \subseteq \mathscr{E}$, and Frobenius action. In fact. $\left(\mathscr{E}, \nabla_{\mathscr{E}}\right)$ is obtained from $\left(\mathscr{P}, \nabla_{\mathscr{P}}\right)$ simply by pulling back $0 \rightarrow \mathscr{O}_{S} \rightarrow \mathscr{P} \rightarrow \Omega_{S / A} \rightarrow 0$ via $\omega^{\cdot}:\left.\mathscr{O}_{\tilde{L}^{i}} \rightarrow \Omega_{S / A}\right|_{\tilde{L}^{\top}}$. Now $F^{1}(\mathscr{E})$ and $\mathscr{E} / F^{1}(\mathscr{E})$ may be identified with $\mathscr{O}_{\tilde{L}}$. Thus, $\omega$ is precisely the differential obtained by considering the Kodaira-Spencer morphism

$$
F^{1}(\mathscr{E})=\mathscr{O}_{\tilde{L}} \rightarrow \Omega_{\tilde{L} / A} \otimes\left(\mathscr{E} / F^{1}(\mathscr{E})\right)=\Omega_{\tilde{L} / A}
$$

Now let $R_{z}$ be the complete local ring which is the completion at $z$ of $U$. 
Let $R_{z}^{\mathrm{PD}}$ be the $p$-adic completion of the PD-envelope of $R_{z}$ at the augmentation ideal $R_{z} \rightarrow A$ defined by $z$. Now taking the inverse limit of the $u_{\omega, n}$ 's defines a unit

$$
u_{\omega} \in\left(R_{z}^{\mathrm{PD}}\right)^{\times}
$$

whose image in the residue field $k$ is 1 . Thus, we can consider $\log \left(u_{\omega}\right) \in R_{z}^{\mathrm{PD}}$.

On the other hand, let $P_{z}=\mathbb{P}\left(z^{*} \mathscr{E}\right)=\mathbb{P}\left(E_{\omega}\right)$. Let $\sigma_{F}: S \rightarrow P_{z}$ (respectively, $\sigma_{V}: S \rightarrow P_{z}$ ) denote the section determined by the subspace $E_{F} \subseteq E_{\omega}$ (respectively, $E_{V} \subseteq E_{\omega}$ ). The trivializations discussed previously define an isomorphism of the tangent space to $P_{z}$ at $\sigma_{F}$ with $A$. Thus, in summary, we get an isomorphism $\phi: P_{z} \cong \mathbb{P}^{1}$ by sending $\sigma_{F}$ (respectively, $\sigma_{V}$ ) to infinity (respectively, zero) and using the trivialization of the tangent space to $\sigma_{F}$ to remove the remaining multiplicative ambiguity. Let $P_{R_{z}^{\mathrm{pD}}}=\left.\mathbb{P}(\mathscr{E})\right|_{\mathrm{Spf}\left(R_{2}^{\mathrm{pD}}\right)}$. Then $\nabla_{\mathscr{E}}$ gives an isomorphism

$$
\Xi_{P}: P_{R_{z}^{\mathrm{pD}}} \cong R_{z}^{\mathrm{PD}} \otimes{ }_{A} P_{z}
$$

which, when composed with $\phi$, gives an isomorphism $\mu: P_{R_{2}^{\text {pD }}} \cong \mathbb{P}_{R_{2}^{\text {pp }}}^{1}$. Now by Theorem 1.7. it follows that the Hodge section (defined by $F^{1}(\mathscr{E}) \rightarrow \mathscr{E}$ )

$$
\sigma: \operatorname{Spf}\left(R_{z}^{\mathrm{PD}}\right) \rightarrow P_{R_{z}^{\mathrm{PD}}} \cong \mathbb{P}_{R_{-}^{\mathrm{PD}}}^{1}
$$

is (in terms of the standard coordinate $t$ on $\mathbb{P}^{1}$, which vanishes at zero and has a pole at infinity) simply $\log \left(u_{\omega}\right)$. (Indeed, Theorem 1.7 tells us that this is true after restriction to any $A$-valued point of $R_{z}^{\mathrm{PD}}$; hence it must be true over $R_{z}^{\mathrm{PD}}$.) It thus follows that the pull-back of the differential $d t$ on $\mathbb{P}^{1}$ via $\sigma$ is simply $\omega^{\prime} \stackrel{\text { def }}{=} d u_{\omega} / u_{\omega}$. But, tracing through all the definitions, the pull-back of $d t$ via the Hodge section is exactly the Kodaira-Spencer morphism of the Hodge filtration. Thus, we conclude that $\omega=\omega^{\prime}$ over $R_{z}^{\mathrm{PD}}$. On the other hand, it is clear that this implies that $\omega=\omega^{\prime}$ over all of $\widetilde{U}$ (since $\mathscr{O}_{\tilde{L}} \rightarrow R_{z}^{\mathrm{PD}}$ is injective). Thus, we have proven the following

Theorem 1.8. We have $\omega=d u_{\omega} / u_{\omega}=\omega^{\prime}$ over $\widetilde{U}$.

Note that this holds (by descent) without the assumption that $k$ is algebraically closed. 


\section{Canonical Liftings of Points in Characteristic $p$}

Let $\alpha_{1} \in S(A)$ be an $A$-valued point of $S$. Suppose we apply $\Phi$ to $\alpha_{1}$ to obtain an $A$-valued point $\beta_{1} \in S(A)$. Then since $\Phi$ is a Frobenius lifting, it induces zero on the morphism on cotangent spaces modulo $p$. Thus, $\beta_{1}\left(\bmod p^{2}\right)$ depends only on $\alpha_{1}(\bmod p)$. Let $\alpha_{2}=\Phi_{A}^{-1}\left(\beta_{1}\right)$. Thus, $\alpha_{2} \equiv \alpha_{1}(\bmod p)$, and $\alpha_{2}$ depends only on $\alpha_{1}(\bmod p)$. If we then continue in this fashion, defining

$$
\alpha_{\imath+1} \stackrel{\text { def }}{=} \Phi_{A}^{-1} \Phi\left(\alpha_{\imath}\right)
$$

it is clear that $\alpha_{\imath} \equiv \alpha_{1}(\bmod p)$ for all $i \geq 1$, and that the sequence $\left\{\alpha_{\imath}\right\}$ of points in $S(A)$ converges $p$-adically. Let $\alpha_{\infty} \in S(A)$ be the limit of this sequence. Let $\alpha_{0} \in S(k)$ be the reduction of $\alpha_{1}$ modulo $p$. Note that we have

$$
\Phi\left(\alpha_{\infty}\right)=\alpha_{\infty}^{F}
$$

and that, moreover, $\alpha_{\infty}$ is the unique $A$-valued point of $S$ which has this property and is equal to $\alpha_{0}$ modulo $p$.

Definition 1.9. We shall call $\alpha_{\infty}$ the canonical lifting of $\alpha_{0}$. We shall call an $A$-valued point of $S$ which is a canonical lifting of some $k$-valued point a canonical A-valued point of $S$.

All the canonical extensions that we have defined become trivial when restricted to $\alpha_{\infty}$. More precisely,

Proposition 1.10. If $\alpha_{0} \in U(k)$, then the restriction of $u_{\omega}$ to $\alpha_{\infty}$ is 1 .

Proof. Indeed, the Hodge filtration of $\mathscr{P}$ is invariant under $\Phi$, so its restriction to $\alpha_{\infty}$ is still Frobenius invariant. By the theory of filtered Dieudonné modules with Frobenius action over $A=W(k)$, it thus follows that the extension of $p$-divisible groups that one obtains is trivial. Thus, by Kummer theory, $\left.u_{\omega}\right|_{\alpha_{\infty}}=1$.

\section{Canonical Multiplicative Parameters}

Let us assume just in this subsection that $k$ is algebraically closed. Let $z \in$ $S(k)$ be a $k$-valued point of $S$. Let $S_{z}^{\log }$ be the completion of $S^{\log }$ at $z$. Thus, $S_{z}^{\text {log }}$ is Spf of a complete local ring $R_{z}$ which is noncanonically isomorphic to 


$$
A\left[\left[t_{1}, \ldots, t_{d}\right]\right]
$$

with the restriction of the divisor $D$ defined by $t_{1}{ }^{\circ} t_{2}{ }^{\circ} \ldots{ }^{\circ} t_{2}$ (where $i$ may be zero). Then if we restrict $\Omega_{\Phi}^{\text {et }}$ to $S_{z}^{\text {log }}$, we obtain the trivial local system.

Let $\omega \in \Omega_{\Omega \Phi}^{\text {et }}$ have integral residues at all the irreducible components of $D$, and nonzero reduction modulo $p$. Thus, $\omega$ defines a surjection

$$
\omega(-): \Theta_{\Phi}^{\mathrm{et}} \rightarrow \mathbb{Z}_{p}
$$

If we apply $\omega(-)$ to our canonical extension of $p$-divisible groups

$$
0 \rightarrow \Theta_{\Phi}^{\mathrm{et}}(1) \otimes_{\mathrm{z}_{p}} \mathbb{Q}_{p} / \mathbb{Z}_{p} \rightarrow G_{\Phi} \rightarrow \mathbb{Q}_{p} / \mathbb{Z}_{p} \rightarrow 0
$$

we obtain an extension of $\mathbb{Q}_{p} / \mathbb{Z}_{p}$ by $\mathbb{Q}_{p} / \mathbb{Z}_{p}(1)$ over $\left.U_{z} \stackrel{\text { def }}{=} S_{z}\right|_{U}$. By Kummer theory, we thus obtain a "logarithmic unit"

$$
q_{\omega, z} \in R_{z}\left[\frac{1}{t_{1}}, \ldots, \frac{1}{t_{1}}\right]^{\times}
$$

which is well-defined up to multiplication by a Teichmüller representative of an element of $k$. If $\omega$ has residue $e_{j}$ at the component of $D$ defined by $t_{j}$, then the valuation of $q_{\omega, z}$ at $\left(t_{j}\right)$ is equal to $e_{j}$. Indeed, this follows from the formula $d q_{\omega, z} / q_{\omega, z}=\omega$ (of Theorem 1.8).

Next, let us consider $\Phi^{-1}\left(q_{\omega, z}\right)$. Since $\Phi^{-1}$ multiplies $d q_{\omega, z} / q_{\omega, z}=\omega$, as well as the canonical extension of $p$-divisible groups by $p$, it follows that $\mathbb{\Phi}^{-1}\left(q_{\omega, z}\right)=$ $\lambda \cdot q_{\omega, z}^{b}$, for some $\lambda \in\left[k^{\times}\right]$(where the brackets mean "the Teichmüller representative of"). On the other hand, because $\Phi$ is a Frobenius lifting, reducing modulo $p$ shows that $\lambda=1$. Thus, we have that

$$
\Phi^{-1}\left(q_{\omega, z}\right)=q_{\omega, z}^{p}
$$

Definition 1.11. We shall call $q_{\omega, z}$ for such an $\omega$ a canonical multiplicative parameter associated to $\Phi^{\mathrm{log}}$.

\section{Canonical Affine Coordinates}

In this subsection, $k$ need not be algebraically closed. Let $\alpha \in U(A)$ be canonical. Let $\mathscr{A l}^{\alpha}$ be the $p$-adic completion of the PD-envelope of $S$ at the subscheme $\operatorname{Im}(\alpha)$. Let $\epsilon_{\alpha}: \mathscr{A}^{\alpha} \rightarrow A$ be the augmentation that defines the point $\alpha$. 
Let $\mathscr{I}=\operatorname{Ker}\left(\epsilon_{\alpha}\right)$. The $A$-algebra structure, together with the augmentation $\epsilon_{\alpha}$ define a splitting

$$
\mathscr{A}^{\alpha}=A \oplus \mathscr{I}
$$

which we shall call the augmentation splitting of $\mathscr{A}^{\alpha}$. Note that we have an $A$-linear Frobenius action

$$
\Phi_{\mathscr{A}}:\left(\mathscr{A}^{\alpha}\right)^{F} \rightarrow \mathscr{A}^{\alpha}
$$

induced by the Frobenius lifting $\Phi$. Moreover, $\Phi_{\mathscr{A}}$ preserves the augmentation splitting, as well as the ring structure of $\mathscr{A}^{\alpha}$.

Let us consider the slopes of this Frobenius action $\Phi_{\mathscr{A}}$. Clearly, $\Phi_{\mathscr{A}}$ acts on $A \oplus 0 \subseteq \mathscr{A}^{\alpha}$ as $\Phi_{A}$. Next, we note that since $\Phi_{\mathscr{A}}$ is a Frobenius lifting, it maps $\mathscr{I}$ into $p \cdot \mathscr{I}$. Thus, we have

$$
\Phi_{\mathscr{A}}\left(\mathscr{\oiiint}^{[j]}\right) \subseteq p^{j} \cdot \mathscr{I}^{[j]}
$$

where the superscript in brackets denotes the divided power. By the definition of an ordinary Frobenius lifting, $\Omega_{\alpha} \stackrel{\text { def }}{=} \mathscr{I} / \mathscr{I}^{[2]}$ has constant slope one. Thus, if we divide $\Phi_{\mathscr{A}}$ restricted to $\Omega_{\alpha}$ by $p$, we obtain an isomorphism

$$
\left(\Omega_{\alpha}\right)^{F} \rightarrow \Omega_{\alpha}
$$

Next, let us consider the $A$-submodule $\Omega^{\text {can }} \subseteq \mathscr{I}$ which is the closure (in the $p$-adic topology) of the intersection of the images of $\left(\left.\frac{1}{p} \cdot \Phi_{\mathscr{A}}\right|_{\mathscr{f}}\right)^{N}$ (for all $N \geq 1$ ). Since $\mathscr{I} / \mathscr{I}^{[2]}$ has slope 1 , it is clear that the projection $\Omega^{\text {can }} \rightarrow \Omega_{\alpha}$ is surjective. Now let us consider the intersection of $\Omega^{\text {can }}$ with $\mathscr{I}^{[2]}$. Let $\phi=$ $\left(\left.\frac{1}{p} \cdot \Phi_{\mathscr{A}}\right|_{\mathscr{\mathscr { S }}}\right)^{N}(\phi)$, where $\phi \in \mathscr{I}$. If $\phi$ is contained in $\mathscr{g}^{[2]}$ modulo $p^{N}$, then since $\mathscr{I} / \mathscr{I}^{[2]}$ has slope 1 , it follows that $\phi$ must also be contained in $\mathscr{I}^{[2]}$ modulo $p^{N}$. But then $\phi=\left(\left.\frac{1}{p} \cdot \Phi_{\mathscr{A}}\right|_{\mathscr{G}}\right)^{N}(\phi)$ must be zero modulo $p^{N}$. Thus, we conclude that the projection $\Omega^{\text {can }} \rightarrow \Omega_{\alpha}$ must be an isomorphism. Inverting this isomorphism, we thus get a canonical morphism

$$
\kappa_{A}: \Omega_{\alpha} \hookrightarrow \mathscr{A}^{\alpha}
$$

Let $S^{\alpha}$ be the formal scheme which is the $p$-adic completion of the PD-envelope in $S$ of the image of $\alpha \in U(A)$. Let $\Theta_{\alpha}$ be the dual $A$-module to $\Omega_{\alpha}$. Let $\Theta_{\alpha}^{\text {aff }}$ 
be the $p$-adic completion of the $\mathrm{PD}$-envelope at the origin of the affine space modeled on $\Theta_{\alpha}$. Thus, $\Theta_{\alpha}^{\text {aff }}$ is Spf of the $p$-adic completion of the PD-envelope of the symmetric algebra (over $A$ ) of $\Omega_{\alpha}$ at the augmentation ideal. We may then reinterpret the canonical morphism $\kappa_{A}$ as an isomorphism

$$
\mathcal{U}^{\text {can }}: \Theta_{\alpha}^{\text {aff }} \rightarrow S^{\alpha}
$$

We thus see that we have proven the following result :

Theorem 1.12. For every choice of a canonical $\alpha \in U(A)$, we obtain a local uniformization (canonically associated to $\Phi$ )

$$
\mathcal{U}^{\text {can }}: \Theta_{\alpha}^{\text {aff }} \cong S^{\alpha}
$$

of $S$ by the affine space modeled on $\Theta_{\alpha}$.

Definition $\mathbb{1} .13$. We shall call the elements of the image of $\kappa_{A}$ canonical affine parameters associated to $\Phi$ at $\alpha$.

Now let $\left(B, \mathfrak{m}_{B}\right)$ be a local ring with residue field $k$ which is $p^{\text {-adically }}$ complete and has a topologically nilpotent PD-structure on $\mathrm{m}_{B}$. Let $\beta \in S(B)$ be such that $\beta\left(\bmod m_{B}\right) \in S(k)$ is equal to $\alpha(\bmod p) \in S(k)$. Let $S^{\beta}$ be the $p$-adic completion of the PD-envelope of $S \otimes_{A} B$ at the image of $\beta$ in $S(B)$. Thus, $S^{\beta} \cong$ $S^{\alpha} \widehat{\otimes}_{A} B$. Let $\mathscr{B}^{\beta}=\mathscr{A}^{\alpha} \widehat{\otimes}_{A} B$. By tensoring the canonical morphism $\kappa_{A}$ constructed above with $B$, we thus obtain a morphism

$$
\kappa_{B}:\left(\Omega_{\alpha}\right)_{B} \stackrel{\text { def }}{=}\left(\Omega_{\alpha}\right) \otimes_{A} B \rightarrow \mathscr{B}^{B}
$$

Let $\epsilon_{\beta}: \mathscr{B}^{\beta} \rightarrow B$ be the augmentation corresponding to the point $\beta \in S(B)$. Let $\Phi_{\beta}=\operatorname{Ker}(\beta)$. Let

$$
\mathscr{B}^{\beta}=B \oplus \mathscr{I}_{\beta}
$$

be the splitting defined by $\epsilon_{\beta}$ and the $B$-algebra structure on $\mathscr{B}^{\beta}$. Let us consider the projections of $\kappa_{B}$ on these two factors:

$$
\kappa_{B}^{0}:\left(\Omega_{\alpha}\right)_{B} \rightarrow B ; \quad \kappa_{B}^{1}:\left(\Omega_{\alpha}\right)_{B} \rightarrow \Phi_{\beta}
$$

Let $\Omega_{\beta}=I_{\beta} / \mathscr{I}_{\beta}^{[2]}$. If we compose $\kappa_{B}^{1}$ with the projection to $\Omega_{\beta}$, we thus obtain a morphism 


$$
\Psi_{\alpha \beta}:\left(\Omega_{\alpha}\right)_{B} \rightarrow \Omega_{\beta}
$$

which is an isomorphism, since it is an isomorphism modulo $\mathfrak{m}_{B}$, where $\beta$ coincides with $\alpha(\bmod p)$. Let $\Theta_{\beta}$ the dual $B$-module to $\Omega_{\beta}$. Now we may regard $\kappa_{B}^{0}$ as an element of $\left(\Theta_{\alpha}\right)_{B}$; if we apply $\left(\Psi_{\alpha \beta}^{\vee}\right)^{-1}$. then we get an element of $\kappa_{\beta} \in \Theta_{\beta}$, hence $\in \mathfrak{m}_{B} \cdot \Theta_{\beta}$ (since $\epsilon_{\beta} \equiv\left(\epsilon_{\alpha}\right)_{B}$ modulo $\left.\mathfrak{m}_{B}\right)$. On the other hand, if we compose $\kappa_{B}^{1}$ with $\Psi_{\alpha \beta}^{-1}$, we get a morphism $\Omega_{\beta} \rightarrow \mathscr{B}^{\beta}$ which gives us an isomorphism

$$
\mathcal{U}^{\beta}: \Theta_{\beta}^{\mathrm{aff}} \rightarrow S^{\beta}
$$

In summary, we have proven the following result:

Theorem 1.11. For every $\beta \in S(B)$ as above, we obtain a canonical class $\kappa_{\beta} \in$ $\mathrm{m}_{B} \cdot \Theta_{\beta}$, as well as a local uniformization

$$
\mathcal{U}^{\beta}: \Theta_{\beta}^{\mathrm{aff}} \cong S^{\beta}
$$

of $S$ by the affine space modeled on $\Theta_{\beta}$. Moreover, this uniformization is related to the canonical uniformization by tensoring over $A$ with $B$, applying the isomorphism

$$
\left(\Psi_{\alpha \beta}^{\vee}\right)^{-1}:\left(\Theta_{\alpha}\right)_{B} \rightarrow \Theta_{\beta}
$$

and then translating by $\kappa_{\beta}$. Finally, for all $\beta \in S(B)$ whose reductions modulo $\mathrm{m}_{B}$ are equal to $\alpha(\bmod p)$, the correspondence $\beta \mapsto \kappa_{\beta}$ is a bijection of such $\beta$ onto $m_{B} \cdot$ $\Theta_{\beta}$.

Proof. All the statements except the last follow from the way we constructed the objects involved. The last statement about the bijection follows from simply evaluating the canonical uniformization of Theorem 1.12 on $B$-valued points.

Finally, we remark (relative to the analogy between Frobenius liftings and Kähler metrics) that these canonical affine parameters are like canonical coordinates for a real analytic Kähler metric.

\section{The Relationship Between Affine and Multiplicative Parameters}

Let us continue with the notation of the preceding subsection, but let us assume in addition that $k$ is algebraically closed, so that the canonical multiplicative parameters are defined. Let $\omega \in \Omega_{\Phi}^{\text {et }}$ have integral residues at all the irre- 
ducible components of $D$, and nonzero reduction modulo $p$. Since $\Omega_{\Phi}^{\text {et }} \subseteq \Omega_{\alpha}$, we may also regard $\omega$ as an element of $\Omega_{\alpha}$. Then we would like to establish the relationship between the canonical multiplicative parameter $q_{\omega, \alpha}$ and the canonical affine parameter $\kappa_{A}(\omega)$.

First note that by Proposition 1.10, $q_{\omega, \alpha}$ evaluated at $\alpha$ is a Teichmüller representative. Thus, $\log \left(q_{\omega, \alpha}\right) \in \mathscr{A}^{\alpha}$ is zero at $\alpha$, as is $\kappa_{A}(\omega) \in \mathscr{A}^{\alpha}$. Moreover, $d \log \left(q_{\omega, \alpha}\right)=\omega$, by Theorem 1.8. On the other hand, the fact that $d \kappa_{A}(\omega)=\omega$ is a tautology. Thus, $\log \left(q_{\omega, \alpha}\right)$ and $\kappa_{A}(\omega)$ have the same derivative and both vanish at $\alpha$. We thus obtain the following result :

Theorem 1.15. We have $\kappa_{A}(\omega)=\log \left(q_{\omega, \alpha}\right)$ in $\mathscr{A}^{\alpha}$.

\section{§2. Construction of the Canonical Frobenius Lifting}

In this Chapter, we shall denote by $\bar{M}_{g, r}^{\text {log }}$ the $p$-adic formal stack of $r$-pointed stable curves of genus $g$ over $\mathbb{Z}_{\phi p}$. We shall denote the reductions of objects over $\mathbb{Z}_{p}$ to $\mathbb{F}_{p}$ or $\mathbb{Z} / p^{2} \mathbb{Z}$ by means of a subscripted $\mathbb{F}_{p}$ or $\mathbb{Z} / p^{2} \mathbb{Z}$. Let $\left(\overline{\mathcal{N}}_{g, r}^{\text {ord }}\right)_{\mathbf{F}_{p}} \rightarrow\left(\overline{\mathcal{M}}_{g, r}\right)_{\mathbf{F}_{p}}$ be the étale morphism in characteristic $p$ of Chapter II. $\S 3$. Thus, $\left(\overline{\mathcal{N}}_{g, r}^{\text {ord }}\right)_{\mathbf{F}_{b}}$ parametrizes pairs consisting of an $r$-pointed stable curve of genus $g$ in characteristic $p$, together with an ordinary, nilpotent indigenous bundle. Let $\overline{\mathcal{N}}_{g, r}^{\text {ord }} \rightarrow \bar{M}_{g, r}$ be the unique étale morphism of $p$-adic formal schemes that lifts $\left(\overline{\mathcal{N}}_{g, r}^{\text {ord }}\right)_{\mathbf{F}_{p}} \rightarrow\left(\overline{\mathcal{M}}_{g, r}\right)_{\mathbf{F}_{p}}$. Thus, $\overline{\mathcal{N}}_{g, r}^{\text {ord }}$ is a smooth $p$-adic formal scheme over $\mathbb{Z}_{p}$ whose reduction modulo $p$ is $\left(\overline{\mathcal{N}}_{g, r}^{\text {ord }}\right)_{F_{p}}$. Our goal in this Section is to construct a canonical ordinary lifting of Frobenius on $\overline{\mathcal{N}}_{g, r}^{\text {ord }}$.

\section{Modular Frobenius Liftings}

In this subsection, we reinterpret certain constructions from Chapter II, §1, in terms of liftings of the Frobenius morphism on $\left(\overline{\mathbb{M}}_{g, r}\right)_{\mathbf{F}_{p}}$. Let $S \rightarrow\left(\overline{\boldsymbol{M}}_{g, r}\right)_{\mathbf{F}_{p}}$ be étale, and let $S^{\log }$ be the log scheme obtained by pulling back the log structure of $\bar{M}_{g, r}^{\text {log }}$. Thus, in particular, $S$ is smooth over $\mathbb{F}_{p}$. Let $\Phi_{S^{100}}: S^{\text {log }} \rightarrow S^{\text {log }}$ be the absolute Frobenius morphism. Since $\left(\bar{M}_{g, r}\right)_{\mathbf{F}_{p}} \subseteq\left(\bar{M}_{g, r}\right)_{\mathbf{Z} / p^{2} \mathbf{Z}}$ is defined by a nilpotent ideal, the étale morphism $S \rightarrow\left(\overline{\mathcal{M}}_{g, r}\right)_{\mathrm{F}_{b}}$ lifts naturally to an étale morphism $\widetilde{S}$ $\rightarrow\left(\bar{M}_{g, r}\right)_{\mathbf{z} / p^{2} \mathbf{Z}}$. We let $\widetilde{S}^{\log }$ be the log scheme obtained by pulling back the log structure of $\left(\bar{M}_{g, r}\right)_{\mathbf{z} / p^{2} \mathbf{Z}}$. We shall call a Frobenius lifting on $\widetilde{S}^{\text {log }}$ a log morphism $\widetilde{S}^{\text {log }} \rightarrow \widetilde{S}^{\text {log }}$ whose reduction modulo $p$ is equal to $\Phi_{S^{\text {loo }}}$. Note that by assigning to étale morphisms $U \rightarrow S$ the set of Frobenius liftings on $\widetilde{U}^{\text {log }}$, we obtain a sheaf $\mathscr{L}$ on the étale site of $S$, with the natural structure of a torsor over $\Theta_{S^{100}}^{\Phi} \stackrel{\text { def }}{=} \Phi_{S}^{*} \Theta_{S^{\text {loo }}}$, 
where $\Theta_{S^{100}}$ is the dual vector bundle to the sheaf of logarithmic differentials on $S^{\log }$. Moreover, this torsor naturally admits a connection $\nabla \mathscr{L}$ as follows: Consider the sheaf of bianalytic functions $\mathscr{O}_{\&^{\text {bi }}}$ on $S^{\text {log }}$. The image $\mathscr{I}_{\Phi}^{S}$ of the Frobenius morphism $\Phi_{S^{b i}}: \mathscr{O}_{x^{b x}} \rightarrow \mathscr{O}_{s^{b}}$ is equal to $i_{\mathrm{L}}\left(\Phi_{S}^{-1} \mathscr{O}_{S}\right)$, as well as to $i_{\mathrm{R}}\left(\Phi_{S}^{-1} \mathscr{O}_{S}\right)$ (where $i_{\mathrm{L}} i_{\mathrm{R}}: \mathscr{O}_{S} \rightarrow \mathscr{O}_{3^{31}}$ are the left and right injections). Thus, the pull-back of the sheaf $\mathscr{L}$ by either $i_{\mathrm{L}}$ or $i_{\mathrm{R}}$ is equal to the sheaf of liftings of $\mathscr{I}_{\Phi}^{S}$ to a $\mathbf{Z} / p^{2} \mathbf{Z}$-flat subalgebra of $O_{\mathscr{S}^{\text {m. }}}$. This gives a connection $\nabla \mathscr{L}$ on the $\Theta_{S^{100}}^{\Phi}$-torsor $\mathscr{L} \rightarrow S$ which is compatible with the natural connection on $\Theta_{S^{100}}^{\Phi}$ (for which sections of $\Phi_{S}^{-1} \Theta_{S^{\text {1og }}}$ are horizontal); also, one checks easily that $\nabla_{\mathscr{L}}$ is integrable.

Now let us recall the $\Theta_{S^{100}}^{\Phi}$-torsor $\mathscr{D} \rightarrow S$ that we defined in Chapter II, $\S 1$. Let $f^{\text {log }}: X^{\text {log }} \rightarrow S^{\text {log }}$ be the pull-back to $S^{\text {log }}$ of the universal curve over $\left(\overline{\mathcal{M}}_{g, r}^{\text {log }}\right)_{\mathbf{F}_{p}}$. Recall then that $\mathscr{D}$ is the $\Theta_{S^{100}}^{\Phi}$-torsor consisting of liftings of the curve $\left(X^{\mathrm{log}}\right)^{F}=$ $X^{109} X_{S^{100}, \Phi_{\text {Sloo }}} S^{\text {log }} \rightarrow S^{\text {log }}$ to an $\widetilde{S}$-flat curve $\widetilde{Y}^{\text {log }} \rightarrow \widetilde{S}^{\text {log }}$. Note that it follows immediately from the definition of the classifying log stack $\bar{M}_{g, r}^{\text {log }}$ (plus the fact that $S \rightarrow \bar{M}_{g, r}$ is étale) that we have an isomorphism

$$
\alpha: \mathscr{D} \rightarrow \mathscr{L}
$$

of $\Theta_{S^{100}}^{\Phi}$-torsors given by considering the classifying map of the lifting $\widetilde{Y}^{\text {log }} \rightarrow \widetilde{S}^{\text {log }}$ (which is, by definition, a Frobenius lifting on $\widetilde{S}^{\text {log }}$. On the other hand, the theory of Chapter II , $\S 1$, gives a natural connection $\nabla_{\mathscr{D}}$ on $\mathscr{D} \rightarrow S$ as follows. Recall the line bundle $\mathscr{T} \stackrel{\text { def }}{=}\left(\Phi_{X^{100} / S^{100}}\right)^{*}\left(\tau_{X^{100} / S^{100}}\right)^{F}$ on $X^{\text {log }}$. By declaring the sections of the $\tau_{X^{100 / S^{100}}}$ inside the definition of $\mathscr{T}$ to be horizontal, we see that $\mathscr{T}$ gets a natural connection $\nabla_{\mathcal{T}}$ over $X^{\log }$ (i.e., not just in the relative sense for $f^{\text {log }}$ $\left.: X^{\log } \rightarrow S^{\text {log }}\right)$. Thus, the de Rham cohomology module $\mathbb{R}^{1} f_{\mathrm{DR}, *}(\mathscr{T})$ has a Gauss-Manin connection $\nabla_{\mathrm{GM}}$ on $S^{\mathrm{log}}$. By Chapter II. Proposition 1.1, we have a natural surjection $\mathbb{R}^{1} f_{\mathrm{DR}, *}(\mathscr{T}) \rightarrow \mathscr{O}_{S}$. which one verifies easily to be horizontal. Since, by Chapter II , Proposition 1.2, D is just the sheaf of sections of this surjection $\mathbb{R}^{1} f_{\mathrm{DR}, *}(\mathscr{T}) \rightarrow \mathscr{O}_{S}$, it thus follows that, as such, $\mathscr{D}$ gets a natural connection (induced by $\nabla_{\mathrm{GM}}$ ), which we shall call $\nabla_{\mathscr{D}}$.

Proposition 2.1. The isomorphism $\alpha$ is horizontal with respect to $\nabla_{\mathscr{D}}$ and $\nabla_{\mathscr{L}}$.

Proof. Let us denote by $X^{\mathrm{L}}$ (respectively, $X^{\mathrm{R}}$ ) the pull-back of $f^{\text {log }}: X^{\mathrm{log}} \rightarrow$ $S^{\text {log }}$ via $i_{\mathrm{L}}: \mathscr{O}_{S} \rightarrow \mathscr{O}_{s^{\text {bu }}}$ (respectively, $i_{\mathrm{R}}: \mathscr{O}_{S} \rightarrow \mathscr{O}_{S^{\text {su }}}$ ) to $\mathscr{S}^{\mathrm{b} 1}$. Thus, we obtain a diagram over $\&^{\text {bi }}$ : 


$$
X^{\mathrm{L}} \leftarrow \mathscr{K}^{\mathrm{b}_{1}} \rightarrow X^{\mathrm{R}}
$$

Let us denote the left-pointing (respectively, right-pointing) arrow by $\pi_{\mathrm{L}}$ (respectively, $\pi_{R}$ ). Similarly, we have an analogous diagram with tildes, for the various objects over $\mathbb{Z} / p^{2} \mathbb{Z}$. Now consider the image of Frobenius $\mathscr{I}_{\Phi}^{S} \subseteq \mathscr{O}_{.8}$. We also have the image of the absolute Frobenius on $X$, which we denote by $\mathscr{I}_{\Phi}^{X}$ $\subseteq \mathscr{O}_{X^{\circ} \text {. }}$. Note that $\mathscr{I}_{\Phi}^{X}$ actually sits inside both $\mathscr{O}_{\Lambda^{\mathrm{L}}}$ and $\mathscr{O}_{Y^{\mathrm{R}}}$.

Suppose next that we are given a $\mathbb{Z} / p^{2} \mathbb{Z}$-flat subalgebra $\widetilde{\mathscr{I}}_{\Phi}^{S} \subseteq \mathbb{O}_{\mathbb{S}^{\circ}}$ that lifts $\mathscr{I}_{\Phi}^{S}$. This corresponds to a section $\eta$ of $\mathscr{L}^{\mathrm{L}}=\mathscr{L}^{\mathrm{R}}$. (Here the superscript " $L$ "'s and " $R$ "'s denote left and right pull-backs to $\mathscr{S}^{\text {bl }}$, respectively.) The obstruction to lifting $\mathscr{I}_{\Phi}^{X}$ to a $\mathbb{Z} / p^{2} \mathbb{Z}$-flat subalgebra of $\mathscr{O}_{\widetilde{X}^{1}}$ compatible with $\widetilde{I}_{\Phi}^{S}$ defines a class in $\mathbb{R}^{1}\left(f^{\mathrm{L}}\right) *\left(\mathscr{T}^{\mathrm{L}}\right)$, which is, by definition, equal to $\xi[\mathrm{L}] \stackrel{\text { def }}{=}\left(\alpha^{\mathrm{L}}\right)^{-1}(\eta)$. Similarly, we obtain a class $\xi[\mathrm{R}] \stackrel{\text { def }}{=}\left(\alpha^{\mathrm{R}}\right)^{-1}(\eta)$. Note that $\left(\pi_{\mathrm{L}}\right)^{-1}(\xi[\mathrm{L}])=\left(\pi_{R}\right)^{-1}$ $(\xi[R])$. since both classes are the obstruction to lifting $\mathscr{I}_{\Phi}^{X}$ to a $\mathbb{Z} / p^{2} \mathbb{Z}$-flat subalgebra of $\mathscr{O}_{\mathscr{X}}$ compatible with $\widetilde{\mathscr{I}}_{\Phi}^{s}$. Let us call this common class $\xi$ [bi].

Now suppose that $\left(\widetilde{\mathscr{I}}^{\prime}\right)_{\Phi}^{S} \subseteq \mathcal{O}_{\tilde{s}}$ is a $\mathbb{Z} / p^{2} \mathbb{Z}$-flat lifting of $\mathscr{I}_{\Phi}^{S}$. Suppose that this lifting correspondings to a section $\zeta$ of $\mathscr{L}$. If we then take $\eta=\zeta^{\mathrm{L}}$ (in the previous paragraph), we get $\xi[\mathrm{bi}]=\left(\pi_{\mathrm{L}}\right)^{-1}\left\{\left(\alpha^{-1}(\zeta)\right)^{L}\right\}$, and, similarly, if we take $\eta=\zeta^{\mathrm{R}}$, we get $\xi[\mathrm{bi}]=\left(\pi_{\mathrm{R}}\right)^{-1}\left\{\left(\alpha^{-1}(\zeta)\right)^{\mathrm{R}}\right\}$. On the one hand, $\nabla_{\mathscr{D}}\left(\alpha^{-1}(\zeta)\right)$ is computed by subtracting these two $\xi[\mathrm{bi}]$ 's. On the other hand, (by the definition of the $\Theta_{S^{108}}^{\Phi}$ torsor structure on $\mathscr{D}$ ) the difference between these two $\xi$ [bi] 's is the difference between the two classfying morphisms given by the subalgebras $\left\{\left(\widetilde{\mathscr{I}}^{\prime}\right)_{\Phi}^{S}\right\}^{\mathrm{L}}$ and $\left\{\left(\widetilde{\mathscr{I}}^{\prime}\right)_{\Phi}^{S}\right\}^{\mathrm{R}}$ of $\mathscr{O}_{\delta^{\mathrm{b}}}$. But this difference is, by definition, $\nabla_{\mathscr{L}}(\zeta)$. This completes the proof.

Henceforth, we shall identify $(\mathscr{D}, \nabla \mathscr{L})$ with $(\mathscr{L}, \nabla \mathscr{L})$, and call the resulting torsor with connection $\left(\mathscr{D}, \nabla_{\mathscr{D}}\right.$ ) (since the notation $\mathscr{L}$ is more natural for line bundles).

\section{Indigenous Sections of $\mathscr{D}$}

We continue with the notation of the previous subsection. Thus, $S \rightarrow \bar{M}_{g, r}$

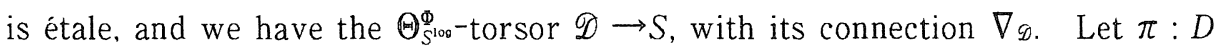
$\rightarrow S$ be the scheme corresponding to this torsor. Thus, $D$ is a twisted version of Spec of the symmetric algebra of the dual of $\Theta_{S^{100}}^{\Phi}$. We endow $D$ with the log structure pulled back from $S$; this gives us a $\log$ stack $D^{\log }$. On $D$, taking the 
dual to the second fundamental exact sequence for differentials gives a sequence of tangent bundles:

$$
0 \rightarrow \Theta_{\left.S^{\log }\right|_{D}} \rightarrow \Theta_{D^{\log }} \rightarrow \Theta_{\left.S^{\log }\right|_{D}} \rightarrow 0
$$

where $\Theta_{D^{100}}$ is the logarithmic tangent bundle on $D^{\text {log }}$. We shall denote the surjection $\left.\Theta_{D^{100}} \rightarrow \Theta_{S^{100}}\right|_{D}$ by $\Theta_{\pi}$. The connection $\nabla_{D}$ then defines a connection $\nabla_{D}$ on the fiber bundle $\pi: D \rightarrow S$, hence a section $\nabla_{\Theta}:\left.\Theta_{S^{\text {100 }}}\right|_{D} \rightarrow \Theta_{D^{100}}$ of $\Theta_{\pi}$.

Now let us suppose that we are given a section $\sigma: S \rightarrow D$ of $\pi$. Then $\sigma$ induces a section of $\sigma^{*} \Theta_{\pi}$, which we denote by $\Theta_{\sigma}: \Theta_{S^{100}} \rightarrow \sigma^{*} \Theta_{D^{100}}$. Also, $\sigma$ defines an FL-bundle $\left(\mathscr{E}, \nabla_{\mathscr{E}}\right)$ (see Chapter II, $\S 1$ ) on the curve $X^{\text {log }}$.

Definition 2.2. We shall call the section $\sigma$ indigenous if the projectivization of the FL-bundle on $X^{\text {log }}$ defined by $\sigma$ is an indigenous bundle on $X^{\text {log }}$.

Let us assume that $\sigma$ is indigenous. Then we obtain, for $i=1,2$, canonical morphisms of vector bundles

$$
\phi_{\imath}: \sigma^{*} \Theta_{D^{\text {log }}} \rightarrow \Theta_{S^{\text {109 }}}
$$

defined functorially as follows. By means of the étale morphism $S \rightarrow \bar{M}_{g, r}$, we can think of the geometric vector bundle $\sigma^{*} \Theta_{D^{\text {wo }}}$ on $S$ as parametrizing infinitesimal deformations $\eta^{\prime}=\left\{\left(X^{\mathrm{log}}\right)^{\prime},\left(\mathscr{E}^{\prime}, \nabla_{\mathscr{g}^{\prime}}\right)\right\}$ of the curve plus FL-bundle pair given by $\eta=\left\{X^{\mathrm{log}},\left(\mathscr{E}, \nabla_{\mathscr{E}}\right)\right\}$. Then the obstruction to lifting the Hodge filtration of $\left.\left(\mathscr{E}^{\prime}, \nabla_{\mathscr{g}^{\prime}}\right)\right|_{X}=\left(\mathscr{E}, \nabla_{\mathscr{E}}\right)$ (which exists since $\sigma$ is indigenous) to a filtration of $\mathscr{E}^{\prime}$

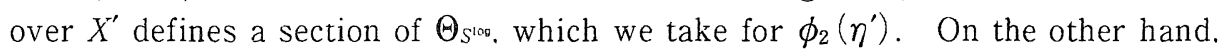
if we think in terms of crystals, then $\left(\mathscr{E}^{\prime}, \nabla_{\mathscr{g}^{\prime}}\right)$ also defines a deformation $\left(\mathscr{E}^{\prime \prime}\right.$, $\left.\nabla_{\mathscr{E}^{\prime}}\right)$ of $\left(\mathscr{E}, \nabla_{\mathscr{G}}\right)$ on $X[\epsilon] /\left(\epsilon^{2}\right)$. The obstruction to lifting the Hodge filtration of $\left(\mathscr{E}, \nabla_{\mathscr{E}}\right)$ to a filtration of $\mathscr{E}^{\prime \prime \prime}$ on $X[\epsilon] /\left(\epsilon^{2}\right)$ defines a section of $\Theta_{S^{100}}$. which we take for $\phi_{1}\left(\eta^{\prime}\right)$. Since $\sigma^{*} \Theta_{\pi}\left(\eta^{\prime}\right)$ is simply the difference between $\left(X^{\mathrm{log}}\right)^{\prime}$ and the trivial deformation of $X^{\text {log }}$, we thus see that

$$
\sigma^{*} \Theta_{\pi}=\phi_{2}-\phi_{1}
$$

We also have that

$$
\phi_{1} \bumpeq \sigma^{*} \nabla_{\Theta}=0
$$

Indeed, sorting through the definitions, one sees that the image of $\nabla_{\theta}$ consists of the $\eta^{\prime}=\left\{\left(X^{\mathrm{log}}\right)^{\prime},\left(\mathscr{E}^{\prime}, \nabla_{\mathscr{E}^{\prime}}\right)\right\}$ obtained by letting $\left(\mathscr{E}^{\prime}, \nabla_{\mathscr{E}^{\prime}}\right)$ be the FL-bundle given 
by regarding $\left(\mathscr{E}, \nabla_{\mathscr{E}}\right)$ as a crystal and taking the bundle with connection that this crystal induces on the deformation $\left(X^{\log }\right)^{\prime}$. Thus, $\left(\mathscr{E}^{\prime \prime}, \nabla_{\mathscr{E}^{\prime}}\right)$ is simply the trivial deformation of $\left(\mathscr{E}, \nabla_{\mathscr{E}}\right)$, hence is indigenous on $X^{\log }$ by assumption. Putting the above two formulas together, we thus obtain that

$$
\phi_{2} \circ \sigma^{*} \nabla_{\Theta}=\mathrm{id}_{\Theta_{S^{\text {10 }}}}
$$

Also, let us note that

$$
\phi_{2} \circlearrowright \Theta_{\sigma}=0
$$

since if it were nonzero, it would measure exactly the extent to which $\sigma$ fails to stay within the indigenous locus of $D$, but, by assumption, $\sigma$ does stay within the indigenous locus.

Next, let us recall the morphism $\Phi_{\mathscr{E}}^{\tau}: \Theta_{S^{100}}^{\Phi} \rightarrow \Theta_{S^{100}}$, i.e., the dual to the "infinitesimal Verschiebung" of Chapter II, $\S 2$. Recall that this morphism was constructed by applying $\mathbb{R}^{1} f_{*}$ to the morphism $\mathscr{T} \rightarrow \tau_{X^{100} / S^{100}}$ given by composing the $p$-curvature $\mathscr{P}: \mathscr{T} \rightarrow \operatorname{Ad}(\mathscr{E})$ of $\mathscr{E}$ with the projection $\operatorname{Ad}(\mathscr{E}) \rightarrow \tau_{x^{100} / S^{100}}$ arising from the Hodge filtration. It thus follows immediately from the definitions (by thinking about how one defines the obstruction that $\phi_{1}$ measures) that if we restrict $\phi_{1}: \sigma^{*} \Theta_{D^{100}} \rightarrow \Theta_{S^{100}}$ to $\Theta_{S^{100}}^{\Phi} \subseteq \sigma^{*} \Theta_{D^{100}}$, we get

$$
\left.\phi_{1}\right|_{\Theta_{S^{100}}^{\phi}}=\Phi_{\mathscr{E}}^{\tau}
$$

So far we have been thinking about morphisms that one can obtain from $\sigma$ by thinking about the indigenous FL-bundle $\left(\mathscr{E}, \nabla_{\mathscr{E}}\right)$ that it defines. But by what we did in the previous subsection, $\sigma$ also defines a Frobenius lifting $\Phi_{\sigma}$ : $\widetilde{S}^{\text {log }} \rightarrow \widetilde{S}^{\text {log }}$. Let us consider the morphism $\Theta_{\Phi_{\sigma}}: \Theta_{S^{109}} \rightarrow \Theta_{S^{100}}^{\Phi}$ obtained by looking at the morphism induced by $\Phi_{\sigma}$ on the tangent bundles, and then dividing by $p$. On the other hand, the morphism $\Theta_{\sigma}-\sigma^{*} \nabla_{\Theta}: \Theta_{S^{\text {lua }}} \rightarrow \sigma^{*} \Theta_{D^{\text {100 }}}$ maps into $\Theta_{S^{100}}^{\Phi} \subseteq$ $\sigma^{*} \Theta_{D^{100}}$. Thus, by abuse of notation, we shall regard $\Theta_{\sigma}-\sigma^{*} \nabla_{\Theta}$ as a morphism $\Theta_{S^{100}} \rightarrow \Theta_{S^{100}}^{\Phi}$. Then we claim that

$$
\Theta_{\Phi_{\sigma}}=\Theta_{\sigma}-\sigma^{*} \nabla_{\Theta}
$$

Indeed, if we think of $\sigma^{*} \nabla_{\Theta}$ as defining a direct sum splitting of $\sigma^{*} \Theta_{D^{100}}$. then $\Theta_{\sigma}$ $-\sigma^{*} \nabla_{\Theta}$ is just the component of $\Theta_{\sigma}$ that sits in the vertical subspace $\Theta_{S^{\circ 0}}^{\Phi_{10}} \subseteq$ $\sigma^{*} \Theta_{D^{100}}$. Put another way, $\Theta_{\sigma}-\sigma^{*} \nabla_{\Theta}: \Theta_{S^{100}} \rightarrow \Theta_{S^{100}}^{\Phi}$ is the Kodaira-Spencer morphism for the section $\sigma$ relative to the connection $\nabla_{D}$. Thus, it follows from the definition of the connection called $\nabla \varphi$ in the previous subsection in terms of 
subalgebras of $\mathscr{O}_{\&^{81}}$ that $\Theta_{\Phi_{\sigma}}=\Theta_{\sigma}-\sigma^{*} \nabla_{\Theta}$.

We are now ready to prove the main technical result of this subsection:

Proposition 2.3. If $\sigma: S \rightarrow D$ is an indigenous secion, then $-\Phi_{\tilde{G}}^{\tau}$ is inverse to $\Theta_{\Phi_{\sigma}}$. In particular, the indigenous bundle associated to $\left(\mathscr{E}, \nabla_{\mathscr{E}}\right)$ is ordinary.

Proof. Indeed, using the various observations made above, we simply compute:

$$
\begin{aligned}
\Phi_{\mathscr{G}}^{\tau}-\Theta_{\Phi_{\sigma}} & =\phi_{1}-\left(\Theta_{\sigma}-\sigma^{*} \nabla_{\Theta}\right) \\
& =\phi_{1} \quad \Theta_{\sigma} \\
& =\left(\phi_{1}-\phi_{2}\right): \Theta_{\sigma} \\
& =-\left(\sigma^{*} \Theta_{\pi}\right): \Theta_{\sigma} \\
& =-\mathrm{id} \Theta_{\Theta^{\operatorname{sos}}}
\end{aligned}
$$

Thus, in particular $\Phi_{\mathscr{E}}^{\tau}$ is an isomorphism, and so $\left(\mathscr{E}, \nabla_{\mathscr{E}}\right)$ defines an ordinary nilpotent indigenous bundle.

\section{Frobenius Invariant Indigenous Bundles}

In this subsection, we change notation slightly. Let $S \rightarrow \overline{\mathcal{N}}_{g, r}^{\text {ord }}$ be an étale morphism of a $p$-adic formal scheme $S$ into $\overline{\mathcal{N}}_{g, r}^{\text {ord }}$. Thus, $S$ is formally smooth over $\mathbb{Z}_{p}$. Also, one may think of $S \rightarrow \overline{\mathcal{N}}_{g, r}^{\text {ord }}$ as the unique étale lifting of its reduction $S_{\mathbf{F}_{b}} \rightarrow\left(\overline{\mathcal{N}}_{g, r}^{\text {ord }}\right)_{\mathbf{F}_{b}}$ modulo $p$. For convenience, we assume that $S_{\mathbf{F}_{p}}$ is affine. Endow $S$ with the log structure pulled back from $\bar{M}_{g, r}^{\text {log }}$. Thus, we get a $p$-adic formal log scheme $S^{\text {log }}$. Pulling back the universal curve over $\bar{M}_{g, r}$, we get a morphism $f^{\log }: X^{\log } \rightarrow S^{\log }$. Let $h^{\log }: Y^{\text {rog }} \rightarrow S^{\log }$ be an $r$-pointed curve of genus $g$ whose reduction modulo $p$ is equal to $\left(X^{\log }\right)_{\mathbf{F}_{p}}^{F} \rightarrow S_{\mathbf{F}_{p}}^{\text {log }}$, i.e., the Frobenius transform of $f_{\mathbf{F}_{p}}^{\text {log }}$. We shall denote the divisor of marked points on $Y$ by $E \subseteq Y$.

Let $n \geq 2$ be a natural number. Suppose that we have a coherent sheaf with connection $\left(\mathscr{F}, \nabla_{\mathscr{F}}\right)$ on $Y^{\mathrm{log}}$, where $\mathscr{F}$ is killed by $p^{n}$ and flat over $\mathbf{Z} / p^{n} \mathbb{Z}$, and the connection $\nabla_{\mathscr{F}}$ is relative to the morphism $h^{\text {log }}: Y^{\text {log }} \rightarrow S^{\text {log }}$. Suppose, moreover, that we are given a filtration $F^{1}(\mathscr{F})_{\mathbf{F}_{p}} \subseteq \mathscr{F}_{\mathrm{F}_{b}}$ of the reduction of $\mathscr{F}$ modulo $p$. We shall call this filtration the Hodge filtration. Then, relative to this data, we define the coherent sheaf with connection $\mathbf{F}^{*}\left(\mathscr{F}, \nabla_{\mathscr{F}}\right)$ as follows. First, we regard $(\mathscr{F}, \nabla \mathscr{F})$ as a crystal on $\operatorname{Crys}\left(Y_{\mathbf{F}_{p}}^{\text {log }} / S^{\text {log }}\right)$. Thus, if we apply the relative Frobenius morphism $\Phi_{X^{100} / S^{100}}$ to this crystal $(\mathscr{F}, \nabla \mathscr{F})$, we obtain a crys$\operatorname{tal}(\mathscr{F}, \nabla \mathscr{F})^{\prime} \stackrel{\text { def }}{=} \Phi_{X^{100} / S^{100}}^{*}(\mathscr{F}, \nabla \mathscr{F})$ on $\operatorname{Crys}\left(X_{\mathrm{F}_{p}}^{\mathrm{log}} / S^{\mathrm{log}}\right)$. Next, we consider the subsheaf $\Phi_{X^{10} / S^{100}}^{*} F^{1}(\mathscr{F})_{\mathbf{F}_{p}} \subseteq \mathscr{F}_{\mathrm{F}_{p}}^{\prime}$. If we then consider the subsheaf of $\left(\mathscr{F}, \nabla_{\mathscr{F}}\right)^{\prime}$ on 
Crys $\left(X_{\mathrm{F}_{p}}^{\log } / S^{\log }\right)$ consisting of sections whose reduction modulo $p$ is contained in

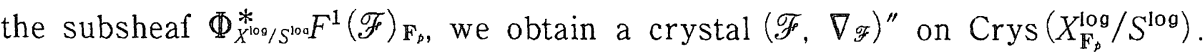
We then let

$$
\mathbb{F}^{*}\left(\mathscr{E}, \nabla_{\mathscr{E}}\right) \stackrel{\text { def }}{=}\left(\mathscr{F}, \nabla_{\mathscr{F}}\right)^{\prime \prime} \bigotimes_{\mathbf{Z}_{\ell}} \mathbb{Z} / p^{n-1} \mathbb{Z}
$$

Definition 2.4. We shall call $\mathbb{F}^{*}\left(\mathscr{F}, \nabla_{F^{*}}\right)$ the renormalized Frobenius pull-back of $(\mathscr{F}, \nabla \mathscr{F})$.

Note that if $\mathscr{F}$ is a vector bundle on $Y \mathrm{Z} / p^{\mathrm{n}} \mathbf{Z}$, and $F^{1}(\mathscr{F})_{\mathrm{F}_{p}}$ is a vector bundle on $Y_{\mathrm{F}_{p}}$, with the injection $F^{1}(\mathscr{F})_{\mathrm{F}_{p}} C \mathscr{F}_{\mathrm{F}_{p}}$ locally split, it follows immediately from the definitions that the coherent sheaf $\mathbb{F}^{*}(\mathscr{F})$ appearing in $\mathbb{F}^{*}(\mathscr{F}, \nabla \mathscr{F})$ is a vector bundle on $X \mathbf{z} / p^{n-1} \mathbf{Z}$. Note also that, if we think of the "input variable" $(\mathscr{F}$, $\left.\nabla_{\mathscr{F}}\right)$ as a crystal on Crys $\left(Y_{\mathrm{F}_{\mathrm{p}}}^{\mathrm{log}} / S^{\mathrm{log}}\right)$, then $\mathbb{F}^{*}\left(\mathscr{F}_{F}, \nabla_{\mathscr{F}}\right)$ does not depend on the

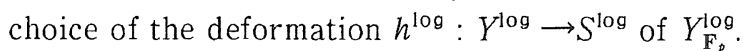

Often, we will be given a Frobenius lifting $\Phi^{\log }: S_{\mathbf{Z} / \rho^{n} \mathbf{Z}}^{\log } \rightarrow S_{\mathbf{Z} / p^{n \mathbf{Z}}}^{\log } \operatorname{modulo} p^{n}$. and we will take $Y_{\mathbf{Z} / p^{n} \mathbf{Z}}^{\log } \rightarrow S_{\mathbf{Z} / p^{n} \mathbf{Z}}^{\log }\left(\right.$ respectively, $\left.\left(\mathscr{F}, \nabla_{\mathscr{F}}\right)\right)$ to be the pull-back of $X_{\mathbf{Z} / p^{n} \mathbf{Z}}^{\log } \rightarrow S_{\mathbf{Z} / p^{n} \mathbf{Z}}^{\log }$ (respectively, some $\left(\mathscr{E}, \nabla_{\mathscr{E}}\right)$ on $X^{\mathrm{log}}$ ) by $\Phi^{\text {log }}$. If $S$ were the spectrum of the ring of Witt vectors of a perfect field $k$, and $n=2$, then the $\mathbb{F}^{*}(\mathscr{E}$. $\left.\nabla_{\mathscr{E}}\right)^{F}$ that we have defined here would coincide with the $\mathbb{F}^{*}\left(\mathscr{E}, \nabla_{\mathscr{E}}\right)$ of Chapter II, Definition 2.9.

Now let $(\mathscr{F}, \nabla \mathscr{F})$ be a vector bundle with connection on $Y_{\mathbf{Z} / r^{n} \mathbb{Z}}^{\log }$ whose determinant is trivial and which is indigenous modulo $p^{n-1}$. We will denote its

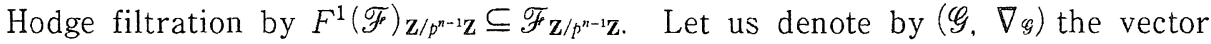
bundle with connection on $X \mathbf{z} / p^{n-1} \mathbf{Z}$ which is the renormalized Frobenius pull-back of $(\mathscr{F}, \nabla \mathscr{F})$. Suppose, moreover. that $(\mathscr{G}, \nabla \mathscr{G}) \underset{\mathrm{F}_{p}}{F} \cong(\mathscr{F}, \nabla \mathscr{F}){ }_{\mathbf{F}_{p}}$. Thus, one sees (as in the proof of Chapter II. Proposition 2.10) that $(\mathscr{F}, \nabla \mathscr{F})_{F_{p}}$ is nilpotent and admissible (hence corresponds, by Chapter II. Proposition 2.5, to some FL-bundle).

Lemma 2.5. Let $n \geq 3$. If we modify the connection $\nabla_{\mathscr{F}}$ by some $p^{n-2} \theta$. whcre $\theta$ is a section of $h_{*}\left(\omega_{Y / S}^{\log }\right) \otimes 2(-E)$, then the vector bundle $\mathbb{F}^{*}(\mathscr{F})$ (on

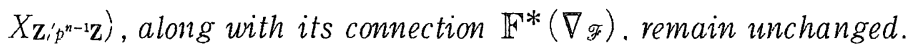

Proof. Looking at the definition of the renormalized Frobenius pull-back, one sees that the pair $\left(\mathbb{F}^{*}(\mathscr{F}), \mathbb{F}^{*}(\nabla \mathscr{F})\right)$ is constructed by pulling back $\mathscr{F}$ (and $\left.\nabla_{\exists}\right)$ via various local liftings of $\Phi_{x}^{100 / S^{100}}$, and then gluing together by means of gluing morphisms defined by the connection $\nabla$ F. Moreover, these gluing morphisms are obtained from the Taylor expansion (cf. [Falt], §2, Theorem $2.3)$, which involves applying the connection $\nabla_{\mathscr{F}}$ to tangent vectors pushed for- 
ward from the Frobenius lifting. Since such tangent vectors are necessarily divisible by $p$ (as well as being annihilated, of course, by $p^{n}$ ), it follows that a knowledge of $(\nabla \mathscr{F})_{\mathbf{Z} / p^{n-1} \mathbf{Z}}$ suffices to compute these Taylor expansions. Thus, certainly $\mathbb{F}^{*}(\mathscr{F})$ depends at most on $\left(\nabla_{\mathscr{F}}\right) \mathbf{z} / p^{n-1} \mathbf{Z}$. On the other hand, since at the end of the construction of $\mathbb{F}^{*}(\mathscr{F})$, we mod out by $p^{n-1} \cdot \Phi_{X^{\text {lus }} / S^{\text {tou }}}^{*} F^{1}(\mathscr{F})_{\mathbf{F}_{p}} \subseteq p^{n-1}$. $\Phi_{\chi^{100} / S^{100}}^{*} \mathscr{F}_{F_{p}}$, we see that modifying $\nabla_{\mathscr{F}}$ by an endomorphism-valued differential whose image lies inside $p^{n-2} \cdot F^{1}(\mathscr{F})$ (where we have $p^{n-2}$ rather than $p^{n-1}$ since we always get an extra factor of $p$ from the fact that we are applying the connection to tangent vectors divisible by $p$ ) does not affect the result. This completes the proof.

Now let us assume that $(\mathscr{F}, \nabla \mathscr{F})$ is a rank two vector bundle on $Y_{Z} \mathrm{Z} / p^{n} \mathbf{Z}$ with a connection (relative to $h^{\text {log }}: Y^{\text {log }} \rightarrow S^{\text {log }}$ ), whose determinant is trivial. Let us suppose, moreover, that $(\mathscr{F}, \nabla \mathscr{F})_{\mathbf{z} / h^{n-1} \mathbf{Z}}$ is indigenous. Let $(\mathscr{G}, \nabla \mathscr{G})=\mathbb{F}^{*}(\mathscr{F}, \nabla \mathscr{F})$. As before, wc assume that $(\mathscr{G}, \nabla \mathscr{G})_{\mathrm{F}_{p}}^{F} \cong\left(\mathscr{F}, \nabla_{\mathscr{F}}\right)_{\mathrm{F}_{p}}$. Then by considering the result of applying $\mathbb{F}^{*}$ to various deformations $\left(\mathscr{F}, \nabla \nabla_{\mathscr{F}}\right)^{\prime}$ of $(\mathscr{F}, \nabla \mathscr{F})$ (i.e., such that $\left.\left(\mathscr{F}, \nabla_{\mathscr{F}}\right)_{\mathbf{Z} / \rho^{n-1} \mathbf{Z}}=\left(\mathscr{F}, \nabla_{\mathscr{F}}\right)^{\prime} \mathbf{Z} / \mathrm{h}^{n-1} \mathbf{Z}\right)$ to obtain various deformations $\left(\mathscr{G}, \nabla_{\mathscr{G}}\right)^{\prime}$ of $(\mathscr{G}$, $\nabla \varphi)$, we obtain a morphism:

$$
\left(\mathbb{R}^{1} f_{\mathrm{DR}, *} \operatorname{Ad}(\mathscr{E})_{\mathbf{F}_{p}}\right)^{F} \rightarrow \mathbb{R}^{1} f_{\mathrm{DR}, *} \operatorname{Ad}(\mathscr{G})_{\mathbf{F}_{p}} \cong \mathbb{R}^{1} f_{\mathrm{DR}, *} \operatorname{Ad}(\mathscr{E})_{\mathbf{F}_{p}}
$$

If we then compose this morphism with the projection

$$
\mathbb{R}^{1} f_{\mathrm{DR}, *} \operatorname{Ad}(\mathscr{\mathscr { E }})_{\mathbf{F}_{p}} \rightarrow \mathbb{R}^{1} f_{*}\left(\tau_{\left.\mathrm{Y}^{1 \ln _{0} / S^{1 \mathrm{I}^{\prime}}}\right)}\right)_{\mathbf{F}_{p}}
$$

arising from the Hodge filtration, we obtain a morphism

$$
\left(\mathbb{R}^{1} f_{\mathrm{DR}, *} \operatorname{Ad}(\mathscr{\varrho})_{\mathbf{F}_{p}}\right) F \rightarrow \mathbb{R}^{1} f_{*}\left(\tau_{\perp^{100 / S^{100}}}\right)_{\mathbf{F}_{o}}
$$

which. by Lemma 2.5, vanishes on the subbundle

$$
\left(f_{*}\left(\omega_{X / S}^{\log }\right) \oslash 2(-D)\right)_{\mathrm{F}_{p}}^{F} \subseteq\left(\mathbb{R}^{1} f_{\mathrm{DR}, *} \operatorname{Ad}(\mathscr{E})_{\mathrm{F}_{p}}\right)^{F}
$$

arising from the Hodge filtration. Thus, we obtain a morphism of vector bundles

$$
\Theta_{\mathbf{F}^{k}}:\left(\mathbb{R}^{1} f_{*}\left(\tau_{\lambda^{100 / S^{100}}}\right)_{\mathrm{F}_{p}}\right) F \rightarrow \mathbb{R}^{1} f_{*}\left(\tau_{X^{100} / S^{100}}\right) \mathbf{F}_{p}
$$

Note that by Lemma 2.5, this morphism remains unchanged if one adds some $p^{n-2} \theta$ to the connection $\nabla \mathscr{F}$. 


\section{Lemma 2.6. The morphism $\Theta_{\mathrm{F}^{*}}$ is equal to $-\bar{\Phi}_{\mathrm{g}}^{\tau}$.}

Proof. In the gluing process referred to in the proof of Lemma 2.5, the deforming cocycle in $\left(\tau_{X^{100} / S^{100}}\right)_{\mathbb{F}_{p}}^{F}=p^{n-1}\left(\tau_{X^{109} / S^{100}}\right)^{F}\left(\bmod p^{n}\right)$ only affects the Taylor ex pansion to first order. Moreover, this cocycle in $\left(\tau_{X^{100} / S^{100}}\right) F_{\mathrm{F}_{p}}$ is mapped to a cocycle in $\Phi_{X^{100 /} / S^{100}}^{*}\left(\tau_{X^{100} / S^{100}}\right) \underset{\mathbf{F}_{p}}{F} \hookrightarrow \operatorname{Ad}(\mathscr{G})_{F_{p}} \cong \operatorname{Ad}(\mathscr{E})_{\mathbf{F}_{p}}$, and hence to a cocycle in $\operatorname{Ad}(\mathscr{E})_{\mathbf{F}_{b}}$. If we then further project this cocycle via $\operatorname{Ad}(\mathscr{E})_{\mathbf{F}_{p}} \rightarrow\left(\tau_{X^{100} / S^{100}}\right)_{\mathbf{F}_{p}}$, we obtain $\Theta_{F^{*}}$ of the original cocycle. On the other hand, let us note that by Chapter II , Proposition 1.4, the inclusion $\Phi_{X^{100} / S^{100}}^{*}\left(\tau_{X^{100 /} / S^{100}}\right) F_{\mathrm{F}_{p}} \hookrightarrow \operatorname{Ad}(\mathscr{G})_{\mathrm{F}_{p}}=\operatorname{Ad}(\mathscr{E})_{\mathrm{F}_{p}}$ is -1 times the $p$-curvature of $\left(\mathscr{E}, \nabla_{\mathscr{E}}\right)_{\mathrm{F}_{p}}$. Since $\Phi_{\mathscr{g}}^{\tau}$ is defined by applying $\mathbb{R}^{1} f_{*}$ to the $p$-curvature composed with the projection $\operatorname{Ad}(\mathscr{E})_{\mathbf{F}_{p}} \rightarrow\left(\tau_{X^{100} / S^{100}}\right)_{\mathbf{F}_{p}}$, we thus obtain the result.

We are now ready to begin a canonical Frobenius lifting on $S^{\text {log }}$, which will be fundamental to the entire paper. First, note that since $\left(\overline{\mathcal{N}}_{g, r}\right)_{\mathbf{F}_{p}} \subseteq\left(\overline{\mathscr{S}}_{g, r}\right)_{\mathbf{F}_{p}}$, we have a tautological trivialization $\left(\tau_{N}\right)_{\mathbf{F}_{p}}:\left(\overline{\mathcal{N}}_{g, r}\right)_{\mathbf{F}_{p}}^{\text {ord }} \rightarrow\left(\overline{\mathscr{S}}_{g, r}\right)_{\mathbf{F}_{p}}$ of the torsor $\overline{\mathscr{\phi}}_{g, r}$ over $\left(\overline{\mathcal{N}}_{g, r}^{\text {ord }}\right)_{\mathbf{F}_{p}}$. If we pull this trivialization back to $S$, we get a trivialization $\left(\tau_{S}\right)_{\mathbf{F}_{p}}$ : $S_{F_{p}} \rightarrow\left(\bar{\phi}_{g, r}\right)_{F_{p}}$, which thus defines a nilpotent, ordinary indigenous bundle $\left(\mathscr{E}, \nabla_{\mathscr{\delta}}\right)_{1}$ on $X_{\mathrm{F}_{p}}^{\mathrm{log}}$. This indigenous bundle thus corresponds to an FL-bundle, hence a section of the torsor $\mathscr{D}$ (of the previous subsection) over $S_{F_{p}}$, and hence a Frobenius lifting $\Phi_{2}^{10 g}: S_{\mathbf{Z} / p^{2} \mathbf{Z}}^{\log } \rightarrow S_{\mathbf{Z} / p^{2} \mathbf{Z}}^{\text {log }} \quad$ Now let $\left(\mathscr{E}, \nabla_{\mathscr{E}}\right)_{2}^{\prime}$ be any indigenous bundle on $X_{\mathbf{Z} / p^{2} \mathbf{Z}}^{\mathrm{log}}$ that lifts $\left(\mathscr{E}, \nabla_{\mathscr{E}}\right)_{1}$. (Such a lifting exists since $S_{\mathbf{F}_{\boldsymbol{p}}}$ is affine.) We shall define $Y^{\text {log }}$ inductively. Let $Y_{\mathbf{Z} / p^{2} \mathbf{Z}}^{10 g}=X_{\mathbf{Z} / p^{2} \mathbf{Z}}^{\log } \times{ }_{S^{100}, \Phi_{2}^{100}} S^{\log }$. Let $(\mathscr{F}, \nabla \mathscr{F})_{2}^{\prime}=$ $\left(\Phi_{2}^{\text {log }}\right) *\left(\mathscr{E}, \nabla_{\mathscr{E}}\right)_{2}^{\prime}$. Then it is a tautology that if we take $\mathbb{F}^{*}\left(\mathscr{F}, \nabla_{\mathscr{F}}\right)_{2}^{\prime}$, we obtain $\left(\mathscr{E}, \nabla_{\mathscr{E}}\right)_{1}$ (up to tensor product with a line bundle with connection whose square is trivial: as usual, for the sake of simplicity, we shall ignore this). So far, to summarize, of the objects constructed so far, $\Phi_{2}^{\log } ; Y_{\mathbf{Z} / p^{2} \mathbf{Z}}^{\mathrm{log}}$ and $\left(\mathscr{E}, \nabla_{\S}\right)_{1}$ are canonical. The primed objects are not canonical.

Let $\left(\mathscr{F}, \nabla_{\mathscr{F}}\right)_{3}^{\prime}$ be any rank two bundle with connection on $Y_{\mathbf{Z} / p^{3} \mathbf{Z}}^{\log }$ whose determinant is trivial, and whose reduction modulo $p^{2}$ is equal to $\left(\mathscr{F}_{F}, \nabla_{\mathscr{F}}\right)_{2}^{\prime}$. That is, $\left(\mathscr{F}, \nabla_{\mathscr{F}}\right)_{3}^{\prime}$ is a deformation of $\left(\mathscr{F}, \nabla_{\mathscr{F}}\right)_{2}^{\prime}$. Now by Lemma 2.6 , and the fact that $\left(\mathscr{E}, \nabla_{\mathscr{E}}\right)_{1}$ is ordinary, it follows that, among all possible deformations $\left(\mathscr{F}, \nabla_{\mathscr{F}}\right)_{3}^{\prime}$ of $(\mathscr{F}, \nabla \mathscr{F})_{2}^{\prime}$, there exists a unique (up to changing the connection by some $p^{2} \cdot \theta$ )

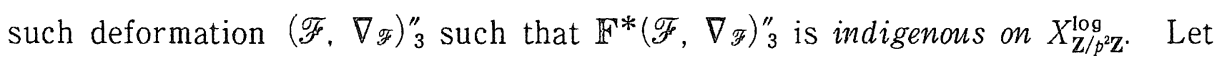
$Y_{\mathbf{Z} / p^{3} \mathbf{Z}}^{\text {log }}$ be the unique deformation of $Y_{\mathbf{Z} / p^{2} \mathbf{Z}}^{\log }$ such that when one evaluates the crystal $\left(\mathscr{F}, \nabla_{\mathscr{F}}\right)_{3}^{\prime \prime}$ on $Y_{\mathrm{Z} / p^{3} \mathrm{Z}}^{\mathrm{jog}}$, it becomes indigenous. Let $\left(\mathscr{E}, \nabla_{\mathscr{E}}\right)_{2}=\mathbb{F}^{*}\left(\mathscr{F}, \nabla_{\mathscr{F}}\right)_{3}^{\prime \prime}$. By Lemma 2.5, $\left(\mathscr{E}, \nabla_{\mathscr{E}}\right)_{2}$ is independent of the choice of $\left(\mathscr{E}, \nabla_{\mathscr{E}}\right)_{2}^{\prime}$ or $\left(\mathscr{F}, \nabla_{\mathscr{F}}\right)_{3}^{\prime \prime}$. Let $(\mathscr{F}, \nabla \mathscr{F})_{2}=\left(\Phi_{2}^{\text {log }}\right)^{*}\left(\mathscr{E}, \nabla_{\mathscr{E}}\right)_{2}$. Let $\Phi_{3}^{10 g}: S_{\mathbf{Z} / p^{3} \mathbf{Z}}^{\log } \rightarrow S_{\mathbf{Z} / p^{3} \mathbf{Z}}^{\text {log }}$ be the classifying morphism of the $r$-pointed stable curve of genus $g$ given by $Y_{\mathbf{Z} / p^{3} \mathbf{Z}}^{\log } \rightarrow S_{\mathbf{Z} / p^{3} \mathbf{Z}}^{\log }$ Thus, $\Phi_{3}^{100}$ lifts 
$\Phi_{2}^{\log }$. Again, to summarize, the objects $\Phi_{3}^{\text {log }} ; Y_{\mathbf{Z} / p^{3} \mathbf{Z}}^{\text {log }}$ and $\left(\mathscr{E}, \nabla_{\mathscr{E}}\right)_{2}$ (as well as $(\mathscr{F}$, $\left.\left.\nabla_{\mathscr{F}}\right)_{2}\right)$ are canonical. If we now let $\left(\mathscr{E}, \nabla_{\mathscr{E}}\right)_{3}^{\prime}$ be an indigenous bundle on $X_{\mathbf{Z} / p^{3} \mathbf{Z}}^{\text {log }}$ that lifts $\left(\mathscr{E}, \nabla_{\mathscr{E}}\right)_{2}$, and $\left(\mathscr{F}, \nabla_{\mathscr{F}}\right)_{3}^{\prime}=\left(\Phi_{3}^{\text {log }}\right) *\left(\mathscr{E}, \nabla_{\mathscr{E}}\right)_{3}^{\prime}$ (where the primed bundles are newly chosen here, hence different from the temporary ones we chose before), it follows from Lemma 2.5 that $\left(\mathscr{E}, \nabla_{\mathscr{E}}\right)_{2} \cong \mathrm{F}^{*}\left(\mathscr{F}, \nabla_{\mathscr{F}}\right)_{3}^{\prime}$.

Continuing in this fashion (making repeated use of Lemmas 2.5 and 2.6, as well as the fact that $\left(\mathscr{E}, \nabla_{\mathscr{E}}\right)_{1}$ is ordinary), we thus obtain a canonical Frobenius lifting $\Phi^{\log }: S^{\log } \rightarrow S^{\log }$ (of $p$-adic formal schemes), as well as a canonical indigenous bundle $\left(\mathscr{E}, \nabla_{\mathscr{E}}\right)$ on $X^{\mathrm{log}}$ such that $\mathbb{F}^{*}\left(\Phi^{\mathrm{log}}\right)^{*}\left(\mathscr{E}, \nabla_{\mathscr{E}}\right) \cong\left(\mathscr{E}, \nabla_{\mathscr{E}}\right)$ (up to tensor product with a line bundle with connection whose square is trivial). Moreover, note that by Proposition 2.3. this Frobenius lifting $\Phi^{109}$ is ordinary.

Definition 2.7. Let $\Psi^{\text {log }:} S^{\log } \rightarrow S^{\log }$ be a Frobenius lifting. We shall call an indigenous bundle $(\mathscr{G}, \nabla \xi)$ on $X^{\log }$ Frobenius invariant for $\Psi^{\log }$ if $\left(\mathscr{G}, \nabla_{\mathscr{G}}\right) \cong$ $\mathbb{F}^{*}\left(\Psi^{\mathrm{log}}\right) *(\mathscr{G}, \nabla \mathscr{G})$ (up to tensor product with a line bundle with connection whose square is trivial).

So far. we have been working over our affine scheme $S$, which is étale over $\bar{M}_{g, r}$. However, since the objects that we have constructed (namely, $\Phi^{\log }$ and $(\mathscr{E}, \nabla:)$ are canonical, i.e. uniquely characterized by certain properties that have nothing special to do with $S$, it is clear that they all descend to $\left(\overline{\mathcal{N}}_{g, r}^{\text {ord }}\right)^{\text {10o }}$. We thus see that we have proven the following key result:

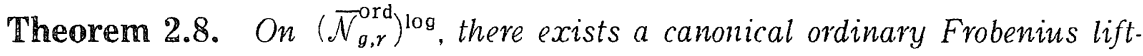
ing

$$
\Phi_{\mathcal{N}}^{\text {log }}:\left(\overline{\mathcal{N}}_{g, r}^{\text {ord }}\right)^{\log } \rightarrow\left(\overline{\mathcal{N}}_{g, r}^{\text {ord }}\right)^{\log }
$$

together with a canonical indigenous bundle $\left(\mathscr{E}_{\mathcal{N}}, \nabla_{\mathscr{E}_{N}}\right)$ on $\mathscr{C}^{\mathrm{log}}$ (where $\mathscr{C}^{\mathrm{log}} \rightarrow$ $\overline{\mathcal{N}}_{g, r}^{\text {ord }}$ is the universal r-pointed curve of genus $g$ ) whose reduction modulo $p$ is equal to the nilpotent, ordinary indigenous bundle defined by the tautological trivialization $\left(\tau_{\mathcal{N}}\right)_{\mathbf{F}_{p}}$ of $\overline{\mathscr{\bigotimes}}_{g . r}$ over $\overline{\mathcal{N}}_{g, r}^{\text {ord }}$. Moreover, the pair $\left\{\Phi_{\mathcal{N}}^{\text {log }} ;\left(\mathscr{E}_{\mathcal{N}}, \nabla_{\mathscr{E}_{\mathcal{N}}}\right)\right\}$ is uniquely characte. rized by the following properties:

(1) $\Phi_{\mathcal{N}}^{\text {log }}:\left(\overline{\mathcal{N}}_{g, r}^{\text {ord }}\right)^{\log } \rightarrow\left(\overline{\mathcal{N}}_{g, r}^{\text {ord }}\right)^{\log }$ is a lifting of Frobenius;

(2) the reduction of $\left(\mathscr{E}_{\mathcal{N}}, \nabla_{\mathscr{E}_{N}}\right)$ modulo $p$ is the bundle defined by $\left(\tau_{\mathcal{N}}\right)_{\mathbf{F}_{p}}$;

(3) $\left(\mathscr{E}_{\mathcal{N}}, \nabla_{\mathscr{E}_{. N}}\right)$ is Frobenius invariant for $\Phi_{\mathcal{N}}^{\text {log }}$. 
Moreover, the formation of $\bar{\Phi}_{\mathcal{N}}^{10 g}$ and $\left(\mathscr{E}_{\mathcal{N}}, \nabla_{\mathbb{B}^{\prime}}\right)$ is compatible with restriction to products of $\overline{\mathcal{N}}_{g, r}^{\text {ord, }}$ f for smaller $g$ 's and r's that map into the boundary of our original $\overline{\mathcal{N}}_{g, r}^{\text {ord }}$ via the gluing procedure described at the end of Chapter I, $\S 2$.

Proof. We have proven everything except the last statement about restriction. To see this, note first of all that $\Phi_{\mathcal{N}}^{\text {log }}$ respects such products of smaller $\overline{\mathcal{N}}_{g, r}^{\text {ord }}$ since it respects the log structure of the original $\overline{\mathcal{N}}_{g, r}^{\text {ord }}$. Thus, we may restrict $\Phi_{\mathcal{N}}^{\log }$ and $\left(\mathscr{E}_{\mathcal{N}}, \nabla_{\mathscr{E}_{N}}\right)$ to these products, and the result follows by uniqueness.

Remark. This result is the central result of this paper. In some sense, the rest of the paper is just devoted to making explicit a number of formal consequences of Theorem 2.8. In particular, since this canonical Frobenius lifting is ordinary, it follows that we can apply the theory of $\S 1$. We shall proceed to do this in the remainder of this Chapter.

Finally, it is useful to know that the formation of the canonical Frobenius and indigenous bundle are compatible with finite coverings. Suppose that $S^{\text {log }}$ $\rightarrow\left(\overline{\mathcal{N}}_{g, r}^{\text {ord }}\right)^{\log }$ is $\log$ etale, with $S$ formally smooth over $\mathbb{Z}_{p}$, and the log structure given by a relative divisor with normal crossings over $\mathbb{Z}_{p}$. Let $f^{\log }: X^{\log } \rightarrow S^{\text {log }}$ be the pull-back of the universal curve over $\bar{M}_{g, r}^{\text {ord }}$. Let $q, s \geq 0$ be such that $2 q$ $-2+s \geq 1$. Let $Y^{\log } \rightarrow S^{\log }$ be an $s$-pointed stable curve of genus $q$. Suppose that we are given a morphism over $S^{\text {log: }}$

$$
Y^{\log } \stackrel{\phi^{100}}{\longrightarrow} X^{\log }
$$

Now we make the following:

Definition 2.9. We shall say that $\phi^{\log }$ is $\log$ admissible if it is finite, log étale, and takes marked points to marked points.

A typical example of a log admissible morphism may be obtained by considering the "admissible coverings" of [HM]. Indeed, it is not difficult to see that by endowing the curves involved (as well as the base) with appropriate log structures, one may obtain a $\log$ admissible covering (cf. [Mzk], §3). (Note, however, that the definition of "log admissible" given here differs from that of [Mzk], §3.)

Let $\left(\mathscr{E}, \nabla_{\mathscr{E}}\right)$ be the restriction of the canonical indigenous bundle $\left(\mathscr{E}_{\mathcal{N}}, \nabla_{\mathscr{E}_{1}}\right)$ to $X^{\text {log }}$. Let $\Phi^{\log }: S^{\log } \rightarrow S^{\text {log }}$ be the pull-back of the Frobenius lifting $\Phi_{\mathcal{N}}^{\text {log }}$ to 
$S^{\log }$ (which exists because $S^{\log } \rightarrow\left(\overline{\mathcal{N}}_{g, r}^{\text {ord }}\right)^{\log }$ is $\log$ étale $)$. Let $(\mathscr{F}, \nabla \mathscr{F})=\phi^{*}\left(\mathscr{E}, \nabla_{\mathscr{E}}\right)$. Observe that $\left(\mathscr{F}, \nabla_{\mathscr{F}}\right)_{\mathbf{F}_{b}}$ is a nilpotent, admissible indigenous bundle on $Y^{\text {log }}$. Let us assume that:

(*) $\left(\mathscr{F}, \nabla_{\mathscr{H}}\right)_{\mathbf{F}_{p}}$ is ordinary.

Then $\left(\mathscr{F}, \nabla \mathscr{F}_{\mathbf{F}_{\boldsymbol{p}}}\right.$ determines a factorization of the classifying morphism $S^{\mathrm{log}} \rightarrow$ $\bar{M}_{q, s}^{\text {iog }}$ through $\left(\overline{\mathcal{N}}_{q, s}^{\text {ord }}\right)^{\text {log }}$. Thus, we get a morphism

$$
\kappa^{\log }: S^{\log } \rightarrow\left(\overline{\mathcal{N}}_{q, S}^{\text {ord }}\right)^{\log }
$$

For simplicity, let us write $T^{\log }$ for $\left(\overline{\mathcal{N}}_{q, s}^{\text {ord }}\right)^{\log }$. Let us denote by $\Psi^{\text {log }}$ the canonical Frobenius on $\left(\overline{\mathcal{N}}_{q, s}^{\text {ord }}\right)^{\log }$, and by $\left(\mathscr{G}, \nabla_{\mathscr{G}}\right)$ the canonical indigenous bundle on the universal $s$-pointed stable curve of genus $q$ over $\left(\bar{N}_{q, s}^{\text {ord }}\right)^{\text {log }}$. Then we have the following compatibility result:

Theorem 2.10. We have a commutative diagram:

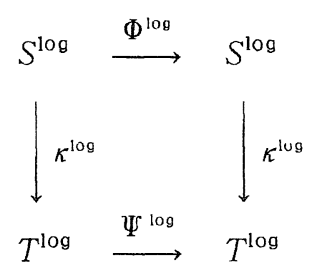

and an isomorphism $\kappa^{*}(\mathscr{G}, \nabla G) \cong\left(\mathscr{F}, \nabla_{\mathscr{F}}\right)$

Proof. We shall apply induction on $i$ to the proposition "the Theorem is true when the objects in it are reduced modulo $p^{l}$." The case $i=1$ is clear. Thus, it suffices to prove the induction step. Let us consider the crystals ( $\mathscr{F}$. $\left.\nabla_{\mathscr{F}}\right)^{\Phi}$ and $\kappa^{*}\left(\mathscr{G}, \nabla_{\mathscr{G}}\right)^{\Psi}$ on $\operatorname{Crys}\left(X_{\mathrm{F}_{p}}^{\mathrm{log}} / S^{\mathrm{log}}\right)$. Suppose that they agree modulo $p^{2}$. If we apply $\mathbb{F}^{*}$ (the renormalized Frobenius pull-back) to them, we get the same crystal modulo $p^{2}$, by the induction hypothesis and the definition of the canonical Frobenii and indigenous bundles. Thus, by Lemma 2.6, it follows that the underlying vector bundles of $\left(\mathscr{F}, \nabla_{\mathscr{F}}\right)^{\Phi}$ and $\kappa^{*}\left(\mathscr{G}, \nabla_{\mathscr{G}}\right)^{\Psi}$ must agree mudulo $p^{l+1}$. Since $(\mathscr{F}, \nabla \mathscr{F})^{\Phi}$ is indigenous on $\left(Y^{\log }\right)^{\Phi}$, and $\kappa^{*}(\mathscr{G}, \nabla \mathscr{G})^{\Psi}$ is indigenous on the $s$-pointed stable curve of genus $q$ given by pulling back the universal one by $\Psi^{109}=\kappa^{109}$, we thus obtain that the diagram in the Theorem commutes modulo $p^{2+1}$. Then since $(\mathscr{F}, \nabla \mathscr{F})$ and $\kappa^{*}(\mathscr{G}, \nabla \mathscr{g})$ agree modulo $p^{2}$, it follows that their underlying vector bundles agree modulo $p^{i+1}$. By a similar argument, their 
underlying vector bundles also agree modulo $p^{i+2}$, and the diagram commutes modulo $p^{\imath+2}$. Then, by Lemma 2.5 , since $\left(\mathscr{F}, \nabla_{\mathscr{F}}\right) \cong \mathbb{F}^{*}\left(\mathscr{F}, \nabla_{\mathscr{F}}\right)^{\Phi}$ and $\kappa^{*}(\mathscr{G}, \nabla \mathscr{G})$ $\cong \kappa^{*} \mathbb{F}^{*}(\mathscr{G}, \nabla \mathscr{G})^{\Psi}$, it follows that $(\mathscr{F}, \nabla \mathscr{F})$ and $\kappa^{*}(\mathscr{G}, \nabla \mathscr{G})$ agree modulo $p^{i+1}$. This completes the proof of the induction step.

\section{§3. Applications of the Canonical Frobenius Lifting}

In this Section, we apply the general theory of $\$ 1$ to the canonical modular Frobenius lifting constructed in $\$ 2$. In particular, we define the notion of a p-adic quasiconformal equivalence class, and show how the choice of such a class allows one to construct both affine and multiplicative uniformizations of $\bar{M}_{g, r}$. We will also define the notion of a p-adic topological marking, which will allow us to construct a local uniformization of $\mathcal{M}_{g . r}$ by means of the affine space of quadratic differentials. As we make these constructions, we will compare them to various classical constructions in the complex case. Finally, we will specialize what we have done in this Chapter to the case of elliptic curves (regarded parabolically) to see that in this case, the canonical Frobenius lifting corresponds to a well-known Frobenius lifting from Serre-Tate theory, and that, consequently, the various objects constructed from it - i.e., canonical curves, modular uniformizations, etc. - reduce to the corresponding objects of classical Serre-Tate theory.

\section{Camonical Liftings of Cunves over Witt Vectors}

Let $\overline{\mathcal{N}}_{g, r}^{\text {ord }} ; \Phi_{\mathcal{N}}^{\text {log }}$ be as in the last subsection of $\S 2$. Let $k$ be a perfect field of characteristic $p$. Let $A=W(k)$, the ring of Witt vectors with coefficients in $k$; let $S=\operatorname{Spec}(A)$. Thus, we have a natural Frobenius automorphism $\Phi_{A}: A \rightarrow A$ on $A$. Recall the notion of canonical liftings of $A$-valued points in $\overline{\mathcal{N}}_{g, r}^{\text {ord }}$ (Definition 1.9).

Definition 3.1. We shall call a point $\alpha_{0} \in \overline{\mathcal{N}}_{g, r}^{\text {ord }}(k)$ a $(p$-adic) quasiconformal equivalence class (valued in $k$ ). We shall call an $r$-pointed stable curve of genus $g$ the canonical curve in the class $\alpha_{0}$ if it admits an indigenous bundle such that the pair consisting of the curve and this indigenous bundle defines a canonical $A$-valued point of $\overline{\mathcal{N}}_{g, r}^{\text {ord }}$ whose reduction modulo $p$ is $\alpha_{0}$.

Remark. Thus, a $p$-adic quasiconformal equivalence class consists of a hyperbolically ordinary $r$-pointed stable curve $\left(X_{0} \rightarrow \operatorname{Spec}(k) ; p_{1}, \ldots, p_{r}: \operatorname{Spec}(k) \rightarrow\right.$ $X_{0}$ ) of genus $g$, together with a choice of a nilpotent, ordinary indigenous bundle 
$\left(\mathscr{E}, \nabla_{\mathscr{E}}\right)_{0}$ on $X_{0}^{\text {log }}$. Recall from Chapter II , Proposition 3.13, that for a given ordinary $X_{0}^{\log }$, there are at most $p^{3 g-3+r}$ possible choices for $\left(\mathscr{E}, \nabla_{\mathscr{E}}\right)_{0}$. The reason for attaching the term "quasiconformal" to this data will become more and more apparent as we continue: Namely, unlike the complex case in which, once $g$ and $r$ are determined, all curves belong to the same quasiconformal equivalence class. the uniformization theory that we shall develop in this paper in the $p$-adic setting acts (by comparison to the classical complex case) as if there are many different quasiconformal equivalence classes (for a given $g$ and $r$ ), and moreover, this equivalence class is determined exactly by the datum of a point in $\overline{\mathcal{N}}_{g, r}^{\text {ord }}$.

Specializing the theory of $\S 1$, we obtain:

Theorem 3.2. For every p-adic quasiconformal equivalence class $\alpha_{0} \in$ $\overline{\mathcal{N}}_{g, r}^{\text {ord }}(k)$, there exists a canonical lifting $\alpha_{\infty} \in \overline{\mathcal{N}}_{g, r}^{\text {ord }}(A)$, i.e., more concretely, an $r$-pointed stable curve $\left(X \rightarrow \operatorname{Spec}(A): p_{1}, \ldots, p_{r}: \operatorname{Spec}(A) \rightarrow X\right)$ of genus $g$, together with an indigenous $\left(\mathscr{E}, \nabla_{\mathscr{E}}\right)$ on $X^{\mathrm{log}}$. This canonical lifting $\alpha_{\infty}$ is uniquely char. acterized by the fact that it is fixed under $\Phi_{A}^{-1} \Phi_{N}^{\text {log }}$, where $\Phi_{\mathcal{N}}^{\text {log }}$ is the canonical Frobenius lifting of Theorem 2.8 .

Corollary 3.3. Suppose that the pair

$$
\left\{\left(X \rightarrow \operatorname{Spec}(A) ; p_{1}, \ldots, p_{r}: \operatorname{Spec}(A) \rightarrow X\right) ;\left(\mathscr{E}, \nabla_{\mathscr{B}}\right)\right\}
$$

is canonical (i.e., for $\left(\mathscr{E}, \nabla_{\varepsilon}\right)$, this means that it is the restriction of the $\left(\mathscr{E}_{\mathcal{N}}, \nabla_{\mathscr{E}_{1}}\right)$ of Theorem 2.8). Then

(1) If $X \rightarrow \operatorname{Spec}(A)$ is smooth, then $\left(\mathscr{E}, \nabla_{\mathscr{E}}\right)$ defines an $M \mathscr{F}^{\nabla}$-object on $X^{\log }$ in the sense of [Falt]. $\S 2$ (up to tensor product with a line bundle whose square is trivial).

(2) More generally, if $X \rightarrow \operatorname{Spec}(A)$ is not smooth, then the pair is obtained by gluing together (as at the end of Chapter $I, \S 2$ ) a collection of smooth canonical pairs.

Proof. The two statements follows by specializing Theorem 2.8.

Corollary 3.4. A pair

$$
\left\{\left(X \rightarrow \operatorname{Spec}(A) ; p_{1}, \ldots, p_{r}: \operatorname{Spec}(A) \rightarrow X\right) ;\left(\mathscr{E}, \nabla_{\mathscr{E}}\right)\right\}
$$

consisting of a smooth r-pointed curve of genus $g$ and an indigenous bundle on $X^{\text {log }}$ 
is canonical if and only if

(1) the indigenous bundle $\left(\mathscr{E}, \nabla_{\mathscr{g}}\right)_{\mathbf{F}_{p}}$ is ordinary;

(2) $\left(\mathscr{E}, \nabla_{\mathscr{E}}\right)$ defines an $\mathcal{M} \mathscr{F}^{\nabla}$-object on $X^{\mathrm{log}}$ in the sense of $[\mathrm{Falt}], \S 2$ (up to tensor product with a line bundle whose square is trivial).

More generally, a pair consisting of an r-pointed stable curve of genus $g$ and an indigenous bundle on $X^{\mathrm{log}}$ is canonical if and only if it is obtained by gluing together canonical pairs as at the end of Chapter I. $\$ 2$.

Proof. Let us first consider the smooth case. By the previous Corollary, it suffices to prove the "if" part. Since $\left(\mathscr{E}, \nabla_{\mathscr{E}}\right)$ is an $\mathcal{M} \mathscr{F}^{\nabla}$-object, we know (by Chapter II , Proposition 2.10) that $\left(\mathscr{E}, \nabla_{\mathscr{E}}\right)_{\mathrm{F}_{\mathrm{b}}}$ is nilpotent. Thus, there exists a canonical pair which is equal to our given pair modulo $p$. On the other hand, it follows by the analogues of Lemmas 2.5 and 2.6 for $\mathbb{F}^{*}$ over $S=\operatorname{Spec}(A)$ that there is only one lifting of our pair modulo $p$ that admits an indigenous bundle which is an $\mathcal{M} \mathscr{F}^{\nabla}$-object. Thus, our pair must be the canonical pair. Next, we consider the stable case. Again, by the previous Corollary, it suffices to prove the "if" part. As before, we note that there exists a canonical pair which is equal to our given pair modulo $p$. By the previous Corollary, the canonical pair is obtained by gluing together smooth canonical pairs. Since a smooth canonical lifting is unique, it thus follows that our pair must be the canonical pair.

Corollary 3.5. Suppose that we have an r-pointed (respectively, s-pointed) stable curve $X^{\mathrm{log}}$ (respectively, $Y^{\mathrm{log}}$ ) of genus $g$ (respectively, $q$ ) over $S^{\mathrm{log}}$ (for some appropriate choice of $\log$ structure on $\left.S^{\mathrm{log}}\right)$. Let $\left(\mathscr{E}, \nabla_{\mathscr{E}}\right)$ (respectively, $\left(\mathscr{F}, \nabla_{\mathscr{F}}\right)$ ) be an indigenons bundle on $X^{\mathrm{log}}$ (respectivcly, $\left.Y^{\mathrm{log}}\right)$. Suppose that $\left(\mathscr{F}_{F}, \nabla_{\mathscr{F}}\right)_{\mathbf{F}_{p}}$, is ordinary. Let $\phi^{\mathrm{log}}: Y^{\mathrm{log}} \rightarrow X^{\mathrm{log}}$ be log admissible, and suppose that $(\mathscr{F}, \nabla \mathscr{F})=\phi^{*}(\mathscr{E}$, $\left.\nabla_{\mathscr{E}}\right)$. Then the pair $\left\{X^{\mathrm{log}}:\left(\mathscr{E}, \nabla_{\mathscr{E}}\right)\right\}$ is canonical if and only if the pair $\left\{Y^{\mathrm{log}} ;(\mathscr{F}\right.$, $\left.\left.\nabla_{7}\right)\right\}$ is canonical.

Proof. First note that, by pulling back square differentials and considering $\Phi_{\mathscr{E}}^{\omega}$ and $\Phi_{\mathscr{F}}^{\omega}$, the ordinariness of $\left(\mathscr{F}, \nabla_{\mathscr{F}}\right)_{\mathrm{F}_{\mathrm{p}}}$ implies the ordinariness of $\left(\mathscr{E}, \nabla_{\mathscr{E}}\right)_{\mathrm{F}_{p}}$. The stipulated conditions on $\phi^{\log }$ imply that $X^{\log }$ is obtained by gluing if and only if $Y^{\text {log }}$ is obtained by gluing. We thus reduce to the smooth case. But this follows immediately, by the criterion of the previous Corollary (about the indigenous bundle being an $\mathcal{M} \mathscr{F}^{\nabla}$-object) and the fact that $\mathbb{F}^{*}$ commutes with log étale coverings. 


\section{Canonical Affine Coordinates on $M_{g, r}$}

We maintain the notation of the preceding subsection. Thus, $S=\operatorname{Spec}(A)$; $A=W(k)$; and $k$ is a perfect field of characteristic $p$. Let $\alpha \in \overline{\mathcal{N}}_{g, r}^{\text {ord }}(A)$ be canonical. Since giving a canonical $\alpha \in \overline{\mathcal{N}}_{g, r}^{\text {ord }}(A)$ is equivalent to giving the $p$-adic quasiconformal equivalence class $\alpha(\bmod p) \in \overline{\mathcal{N}}_{g, r}^{\text {ord }}(k)$, we shall frequently abuse notation and speak of "the $p$-adic quasiconformal equivalence class $\alpha$."

Let us assume that $\alpha$ corresponds to a smooth curve. Then applying Theorems 1.12 and 1.14 to the canonical Frobenius lifting of Theorem 2.8 gives the following results:

Theorem 3.6. For every choice of a p-adic quasiconformal equivalence class $\alpha$, we obtain a local canonical uniformization

$$
\mathcal{U}^{\mathrm{can}}: \Theta_{\alpha}^{\mathrm{aff}} \cong \mathcal{M}_{g, r}^{\alpha}
$$

of $\mathcal{M}_{g, r}$ by the affine space modcled on $\Theta_{\alpha}$.

Let $\left(B, \mathrm{~m}_{B}\right)$ be a local ring with residue field $k$ which is $p$-adically complete and has a topologically nilpotent PD-structure on $\mathrm{m}_{B}$.

Definition 3.7. We shall say that $\beta \in \overline{\mathcal{N}}_{g, r}^{\text {ord }}(B)$ is in the ( $p$-adic) quasiconformal equivalence class $\alpha$ if the point $\beta\left(\bmod \mathrm{m}_{B}\right) \in \overline{\mathcal{N}}_{g, r}^{\text {ord }}(k)$ is equal to $\alpha(\bmod p)$ $\in \bar{N}_{g, r}^{\text {ord }}(k)$.

In summary, we have proven the following result:

Theorem 3.8. For every $\beta \in \overline{\mathcal{N}}_{g, r}^{\text {ord }}(B)$ in the quasiconformal equivalence class $\alpha$, we obtain a canonical class $\kappa_{\beta} \in \mathfrak{m}_{B} \cdot \Theta_{\beta}$, as well as a local uniformization

$$
u^{\beta}: \Theta_{\beta}^{\mathrm{aff}} \cong \mathcal{M}_{g, r}^{\beta}
$$

of $\mathcal{M}_{g, r}$ by the affine space modeled on $\Theta_{\beta}$. Moreover, this uniformization is related to the canonical uniformization by tensoring over $A$ with $B$, applying the isomor. phism

$$
\left(\Psi_{\alpha \beta}^{\vee}\right)^{-1}:\left(\Theta_{\alpha}\right)_{B} \rightarrow \Theta_{\beta}
$$

and then translating by $\kappa_{\beta}$. Finally, for all $\beta \in \overline{\mathcal{N}}_{g, r}^{\text {ord }}(B)$ that lie in the quasicon- 
formal equivalence class $\alpha$, the correspondence $\beta \mapsto \kappa_{\beta}$ is a bijection of such $\beta$ onto $\mathfrak{m}_{B} \circ \Theta_{\beta}$

Remark. In considering the uniformizations just obtained, it is tempting to compare them with the local uniformization by the affine space modeled on the tangent space to $\boldsymbol{M}_{g, r}$ given in the complex case by considering geodesics for the Teichmüller metric. We believe, however, that if there is any proper complex analogue to the uniformizations of Theorems 3.6 and 3.8 at all, then it is the uniformization obtained by Bers coordinates. Indeed, unlike the Teichmüller coordinates, which are real, but not complex analytic, the Bers coordinates are (complex) analytic, just as the affine coordinates of Theorems 3.6 and 3.8. Also, (perhaps more crucially) the Teichmüller coordinates are the same for elliptic curves regarded either hyperbolically or parabolically. We shall soon see, however, that the uniformizations analogous to those of Theorems 3.6 and 3.8 for elliptic curves treated parabolically are different from those in the hyperbolic case. One difference between the Bers uniformization and the uniformizations of Theorems 3.6 and 3.8 is that the Bers uniformization is by the affine space of quadratic differentials (of the complex conjugate curve). not by the tangent space to $\boldsymbol{M}_{g, r}$. On the other hand, (even in the complex case) one cannot have a holomorphic local canonical uniformization by the affine space of quadratic differentials, as one can see easily by considering a one-pointed curve of genus one with an automorphism of degree three. Thus, to obtain a uniformization by quadratic differentials, we need more "rigidifying" information. In our case, the information will take the form of a topological marking of the curve.

\section{Topological Markings and Uniformization by Quadratic Differentials}

We maintain the notation of the previous subsection. Let us consider the canonical Frobenius lifting $\Phi_{\mathcal{N}}: \overline{\mathcal{N}}_{g, r}^{\text {ord }} \rightarrow \overline{\mathcal{N}}_{g, r}^{\text {ord }}$ of Theorem 2.8. Just as in Definition $1.2, \Phi_{N}$ defines canonical étale local systems on $\overline{\mathcal{N}}_{g, r}^{\text {ord }}$ in free $\mathbb{Z}_{p}$-modules of rank $3 g-3+r$ :

Definition 3.9. We shall refer to $\Theta_{\mathcal{N}}^{\text {et }}$ as the tangential local system on $\overline{\mathcal{N}}_{g, r}^{\text {ord }}$. We shall call its dual, $\Omega_{N}^{\text {et }}$, the differential local system on $\overline{\mathcal{N}}_{g, r}^{\text {ord }}$.

Note that if one tensors over $\mathbb{Z}_{p}$ with $\mathbb{F}_{p}$, then these local systems become the local systems (with the same names) considered in Chapter II, §3, following Proposition 3.4.

Now let us assume that $k$ is algebraically closed. Let $\alpha \in \overline{\mathcal{N}}_{g, r}^{\text {ord }}(A)$ be a $p$-adic quasiconformal equivalence class. In this subsection, however, we assume that $\alpha$ corresponds to a smooth curve. We would like to consider the 
fundamental groupoid of $\overline{\mathcal{N}}_{g, r}^{\text {rd }}$ in the sense of [SGA 1]. Exposé V, p.130. Recall that this fundamental groupoid is the category of fiber functors from the category of finite étale coverings of $\overline{\mathcal{N}}_{g, r}^{\text {ord }}$ to the category of sets. Moreover, if $x \in$ $\overline{\mathcal{N}}_{g, r}^{\text {ord }}(k)$, then $x$ defines a fiber functor $\mathscr{F}_{x}$ of this type by simply restricting étale coverings of $\overline{\mathcal{N}}_{g, r}^{\text {ord }}$ to $\operatorname{Spec}(k)$ via pull-back by $x$. Thus, if $x, y \in \overline{\mathcal{N}}_{g, r}^{\text {rdd }}(k)$, then we shall call a path from $x$ to $y$ a natural transformation from $\mathscr{F}_{x}$ to $\mathscr{F}_{y}$.

On the other hand, recall from the last subsection of Chapter II . §3, the set $\mathscr{D}_{g, r}$ of isomorphism classes of totally degenerate $r$-pointed stable curves of genus $g$. If $\Delta \in \mathscr{D}_{g, r}$, then, by abuse of notation, we shall also write $\Delta \in \overline{\mathcal{N}}_{g, r}^{\text {ord }}(A)$ for the totally degenerate curve over $A$ represented by $\Delta$. Now we make the following important

Definition 3.10. We shall call a pair $\mu=(\Delta ; \varpi)$ a (p-adic) topological marking for the quasiconformal equivalence class $\alpha$ if $\Delta \in \mathscr{D}_{g . r}$ and $\varpi$ is a path from $\alpha(\bmod p) \in \overline{\mathcal{N}}_{g, r}^{\text {ord }}(k)$ to $\Delta \in \overline{\mathcal{N}}_{g, r}^{\text {ord }}(k)$.

Let $\mu=(\Delta ; \varpi)$ be a topological marking for $\alpha$. Then let us note that $\mu$ defines a canonical nondegenerate bilinear form $\mathscr{B}_{\mu}$ on $\Theta_{\alpha}$ as follows. First note that by the construction in Proposition 3.6 of Chapter II , §3 (which is carried out there over $\mathbf{F}_{p}$, but clearly works just as well over $\mathbf{Z}_{p}$ ), we have a canonical nondegenerate bilinear from $\mathscr{B}_{\Delta}^{\text {et }}$ on $\Theta_{\Delta}^{\text {et }}$. Now the path $\varpi$ defines an isomorphism $\Theta_{\varpi}: \Theta_{\alpha}^{\text {et }} \cong \Theta_{\Delta}^{\text {et }}$. Thus, if we pull-back $\mathscr{B}_{\Delta}^{\text {et }}$ by means of $\Theta_{\varpi}$, we get a canonical nondegenerate bilinear form $\mathscr{B}_{\mu}^{\mathrm{et}}$ on $\Theta_{\alpha}^{\mathrm{et}}$. Since $\Theta_{\alpha}=\left(\Theta_{\alpha}^{\mathrm{et}}\right) \bigotimes_{\mathrm{z}_{p}} A$, we thus obtain (by tensoring) a canonical nondegenerate bilinear form $\mathscr{B}_{\mu}$ on $\Theta_{\alpha}$.

Now let $\left(B, \mathfrak{m}_{B}\right)$ be a local ring with residue field $k$ which is $p$-adically complete and has a topologically nilpotent $\mathrm{PD}$-structure on $\mathfrak{m}_{B}$. Let $\beta \in \overline{\mathcal{N}}_{g, r}^{\text {rd }}(B)$ be in the quasiconformal equivalence class $\alpha$. Recall the canonical isomorphism

$$
\left(\Psi_{\alpha \beta}^{\vee}\right)^{-1}:\left(\Theta_{\alpha}\right)_{B} \rightarrow \Theta_{\beta}
$$

implicit in Theorem 3.8. This isomorphism allows us to transport $\mathscr{B}_{\mu}$ to $\Theta_{\beta}$ so as to obtain a canonical nondegenerate bilinear form $\mathscr{B}_{\mu}^{\beta}$ on $\Theta_{\beta}$. We summarize this as follows:

Proposition 3.11. The choice of a topological marking $\mu$ on a quasiconformal equivalence class $\alpha$ allows one to define a canonical nondegenerate bilinear form $\mathscr{B}_{\mu}^{\beta}$ on $\Theta_{\beta}$ for every $\beta \in \overline{\mathcal{N}}_{g, r}^{\text {rd }}(B)$ in the quasiconformal equivalence class $\alpha$.

This finally allows us to give local uniformizations of $\mathcal{M}_{g, r}$ by means of quadrat- 
ic differentials: Namely, we compose the affine uniformization of Theorem 3.8 with the isomorphism $\Theta_{\beta} \cong \Omega_{\beta}$ given by the nondegenerate bilinear form $\mathscr{B}_{\mu}^{\beta}$.

Theorem 3.12. The choice of a topological marking $\mu$ on a quasiconformal equivalence class $\alpha$ that corresponds to a smooth curve allows one to define a canoni. cal class $\kappa_{\mu, \beta}^{\vee} \in \mathfrak{m}_{B} \cdot \Omega_{\beta}$, as well as a local uniformization

$$
\mathscr{V}_{\mu, \beta}: \Omega_{\beta}^{\mathrm{aff}} \cong \mathcal{M}_{g, r}^{\beta}
$$

of $M_{g, r}$ by the affine space modeled on $\Omega_{\beta}$, for every $\beta \in \overline{\mathcal{N}}_{g, r}^{\text {ord }}(B)$ in the quasiconformal equivalence class $\alpha$. Finally, for all $\beta \in \overline{\mathcal{N}}_{g, r}^{\text {ord }}(B)$ that lie in the quasiconformal equivalence class $\alpha$, the correspondence $\beta \mapsto \kappa_{\mu, \beta}$ is a bijection of such $\beta$ onto $\mathfrak{m}_{B} \cdot \Omega_{\beta}$.

Remark. Thus, we have obtained a canonical uniformization of $\boldsymbol{M}_{g, r}$ by quadratic differentials for every choice of a topological marking on $\alpha$. In the complex case, a topological marking of a Riemann surface is given by fixing the underlying topological manifold, up to homeomorphisms homotopic to the identity. Thus, the analogy between topological markings in the $p$-adic and complex cases lies in the fact that a $p$-adic topological marking gives one a canonical basis for $\Theta_{\beta}^{\text {et }}$, hence for $\Theta_{\beta}$, corresponding to a collection of partition curves (see Introduction, §2) of a Riemann surface. This specification of partition curves determines a topological marking, by gluing together "pants" along the partition curves. Thus, instead of uniformizing by the affine space modeled on $\Omega_{\beta}$, we could also have uniformized by the affine space modeled on a direct product of affine lines, one for each "partition curve." Whichever choice of coordinates (i.e., quadratic differentials or partition curves) is more useful depends on one's tastes or the applications one has in mind.

\section{Camonical Multiplicative Parameters}

So far we have only been working with smooth curves. In order to find canonical parameters at singular curves, we need to work with multiplicative parameters (like the $q$-parameter in the case of elliptic curves), as opposed to affine parameters, as in Theorems 3.6 and 3.8.

Let $\alpha \in \overline{\mathcal{N}}_{g, r}^{\text {ord }}(A)$ be a $p$-adic quasiconformal equivalence class (corresponding to a curve which is not necessarily smooth). Let us assume, for the rest of this subsection, that $k$ is algebraically closed. Let $\Omega_{\alpha}^{\text {log }}$ be the restriction of $\Omega_{\bar{M}_{o r}^{100}}$ to $\alpha$, and let $\Theta_{\alpha}$ be the dual $A$-module to $\Omega_{\alpha}^{\text {log }}$. Then the Frobenius invariant subsections of $\Theta_{\alpha}$ form a free $\mathbb{Z}_{p}$-submodule $\Theta_{\alpha}^{\text {et }} \subseteq \Theta_{\alpha}$ of rank $3 g-3+\gamma$. Similarly, we have $\Omega_{\alpha}^{\text {et }} \subseteq \Omega_{\alpha}^{\text {log }}$. Let $\left(\bar{M}_{g, r}\right)_{\alpha}$ be the completion of $\bar{M}_{g, r} \otimes_{\mathrm{z}_{\diamond}} A$ at 
the image of $\alpha$. Let $\omega \in \Omega_{\alpha}^{\text {et }}$ have residues equal to zero or one at all the irreducible components of the divisor at infinity of $\bar{M}_{g, r}$, and nonzero reduction modulo p. Then, just as in Definition 1.11, we have a parameter

$$
q_{\omega, \alpha}
$$

on $\left(\bar{M}_{g, r}\right)_{\alpha}$, which is well-defined up to multiplication by a Teichmüller representative $\left[k^{x}\right]$. This parameter is a unit at all the divisors where the residue of $\omega$ is zero and has valuation one at all the divisors where the residue of $\omega$ is one. Moreover,

$$
\Phi_{\overline{\mathcal{N}}}^{-1}\left(q_{\omega, z}\right)=q_{\omega, z}^{\natural}
$$

Definition 3.13. We shall call such a parameter $q_{\omega, \alpha}$ a canonical multiplicative parameter on $\left(\bar{M}_{g, r}\right)_{\alpha}$.

\section{The Case of Elliptic Curves}

Just as in previous Chapters. it is useful to look at the case of elliptic curves (regarded parabolically) since the calculations are usually much easier in this case. As before, we let $\bar{M}_{1,0}^{\text {log }}$ be the log stack of elliptic curves, and $f^{\text {log. }}$ $\mathscr{G}^{\text {log }} \rightarrow \bar{M}_{1,0}^{\text {log }}$ be the universal elliptic curve (with the log structure defined by the pull-back to $\mathscr{G}$ of the divisor at infinity of $\left.\bar{M}_{1,0}\right)$. Let $\bar{M}_{1,0}^{\log } \subseteq \bar{M}_{1,0}$ be the open $p$-adic formal substack parametrizing ordinary elliptic curves. Recall that we computed in Chapter II , Theorem 3.11, that $\overline{\mathcal{N}}_{1,0}^{\text {ord }}=\bar{M}_{1,0}^{\text {ord }}$, and that the section of $\overline{\mathscr{S}}_{1,0}$ over $\left(\overline{\mathcal{M}}_{1.0}^{\text {rd }}\right)_{\mathbf{F}_{p}}$ corresponding to the unique nilpotent, ordinary indigenous bundle on an elliptic curve was given explicitly in Example 2 of Chapter I. $\S 2$. Now it is easy to see that, although nominally everything in this Chapter was done for hyperbolic curves, much of the theory goes through for elliptic curves, as well. In particular, the construction of the canonical Frobenius lifting $\Phi_{\mathcal{N}}$ on $\bar{M}_{1,0}^{\text {ord }}$ goes through just as before. Since everything else in the Chapter is essentially a formal consequence of the existence of $\Phi_{\mathcal{N}}$, in this subsection, we would like to compute the lifting $\Phi_{\mathcal{N}}$ explicitly for elliptic curves, and identify the resulting concepts (i.e., canonical curves, uniformization, topological marking, etc.) with the well-known objects of classical Serre-Tate theory. For a treatment of classical Serre-Tate theory, we refer to [Mess] and [KM] (p.260).

Let us begin by recalling a certain Frobenius lifting $\Phi_{\mu}$ on $\bar{M}_{1,0}^{\text {ord }}$ which is fundamental to Serre-Tate theory. Ultimately, we shall show that $\Phi_{\mu}=\Phi_{\mathcal{N}}$. First recall that the etale quotient of the $(\log ) p$-divisible group $\mathscr{P}$ associated to the universal elliptic curve $\mathscr{G}^{\text {ord }} \rightarrow \bar{M}_{1,0}^{\text {ord }}$ defines a local system $\mathscr{L}$ on $\bar{M}_{1,0}^{\text {ord }}$ in free 
$\mathbb{Z}_{p}$-modules of rank one. Also, since $\mathscr{P}$ is self-dual, taking Cartier duals gives us an inclusion $\mathscr{L}^{\vee}(1) \otimes\left(\mathbb{Q}_{p} / \mathbb{Z}_{p}\right) \hookrightarrow \mathscr{P}$ (where the " 1 " in parentheses denotes a Tate twist). Let $\mathscr{P}_{\Phi} \subseteq \mathscr{P}$ be the subgroup scheme given by $\mathscr{L}^{\vee}(1) \otimes\left(\frac{1}{p} \mathbb{Z}_{p} / \mathbb{Z}_{p}\right)$. Thus, taking the quotient by this subgroup scheme $\mathscr{P}_{\Phi}$ gives us an isogeny:

$$
\Phi_{\mathscr{G}}: \mathscr{G}^{\text {ord }} \rightarrow \mathscr{H}
$$

to some elliptic curve $\mathscr{H}$ over $\bar{M}_{1,0}^{\text {ord }}$. Let $\Phi_{\mu}: \bar{M}_{1,0}^{\text {ord }} \rightarrow \bar{M}_{1,0}^{\text {ord }}$ be the classifying morphism of $\mathscr{H}$. Thus, $\mathscr{H}=\Phi_{\mathscr{M}}^{*} \mathscr{G}^{\text {ord }}$. Since considered modulo $p$. the subgroup scheme $\mathscr{P}_{\Phi}$ is nothing but the kernel of Frobenius, it follows that $\Phi_{\mu}$ is a Frobenius lifting, and that $\left(\Phi_{\mathscr{G}}\right)_{\mathbf{F}_{p}}$ is just the relative Frobenius on $\mathscr{G}_{\mathrm{F}_{p}}^{\text {ord }}$. For convenience, we shall denote objects pulled back via $\Phi_{\mathcal{M}}$ with a superscript "F."

Now let us consider the effect of pulling back the indigenous bundle $(\mathscr{E}$, $\left.\nabla_{\mathscr{E}}\right)^{F}$ on $\left(\mathscr{G}^{\text {ord }}\right)^{F}$, where $\left(\mathscr{E}, \nabla_{\mathscr{E}}\right)$ is the indigenous bundle on $\mathscr{G}^{\text {ord }}$ given in Example 2 of Chapter I, $\S 2$. Let $\left(\mathscr{F}_{F}, \nabla \mathscr{F}\right)=\Phi_{\mathscr{G}}^{*}\left(\mathscr{E}_{,}, \nabla_{\mathscr{E}}\right)^{F}$. Let us denote by $\omega$ the relative dualizing sheaf of $\mathscr{G}^{\text {ord }} \rightarrow \bar{M}_{1,0}^{\text {ord }}$. Then as a vector bundle.

$$
\mathscr{F}=\Phi_{\mathscr{G}}^{*}(\omega)^{F} \oplus \mathscr{O}_{\mathscr{G}}
$$

Now let $\Phi_{\mathscr{g}}^{\omega}: \Phi_{\mathscr{G}}^{*}(\omega)^{F} \rightarrow \omega$ denote the morphism on differentials induced by $\Phi_{\mathscr{G}}$, divided by $p$. Then I claim that $\Phi_{\mathscr{G}}^{\omega}$ is an isomorphism. Indeed, since we are dealing with ordinary elliptic curves, the local group structure near the origin is isomorphic to that of $\mathbb{G}_{m}$ (the multiplicative group scheme), and the Frobenius lifting $\Phi_{\mathscr{G}}$ just amounts to the $p^{\text {th }}$ power map on $\mathbb{G}_{m}$. This proves the claim. Since $\mathscr{E}=\omega \oplus \mathcal{O}_{\mathscr{G}}, \Phi_{\mathscr{G}}^{\omega}$ thus gives us an isomorphism:

$$
\mathscr{E} \cong \mathscr{F}
$$

by taking the direct sum of $\Phi_{\mathscr{G}}^{\omega}$ with the identity on $\mathscr{O}_{\xi}$. Next, we consider connections. Recall that $\nabla_{\mathscr{E}}$ differs from the trivial connection by the tautological Ad $(\mathscr{E})$-valued differential form given by mapping the first factor $\omega$ to the second factor $\mathscr{O}_{\mathscr{G}} \otimes \omega$. Thus, when we pull-back by $\Phi_{\mathscr{G}}$, we get a similar nilpotent endomorphism-valued differential form, this time given by the map from $\Phi_{\mathscr{G}}^{*}(\omega)^{F}$ (the first factor) to $\mathcal{O}_{g} \otimes \omega$ (the second factor) given by $p \cdot \Phi_{G}^{\omega}$. On the other hand, when we compute the renormalized Frobenius pull-back of $\left(\mathscr{E}, \nabla_{\mathscr{E}}\right)$, we divide out by this factor of $p$. It thus follows that under the isomorphism $\mathscr{E} \cong \mathscr{F}$ considered above, the renormalized Frobenius pull-back gives a connection on $\mathscr{F}$ which corresponds precisely to the connection $\nabla_{\mathscr{E}}$ on $\mathscr{E}$. Since $\Phi_{\mathcal{N}}$ and $\mathscr{E}_{\mathcal{N}}$, $\left.\nabla_{\mathscr{E}_{N}}\right)$ are uniquely characterized by the property that the renormalized 
Frobenius pull-back of $\Phi_{\mathcal{N}}^{*}\left(\mathscr{E}_{\mathcal{N}}, \nabla_{\mathscr{E}_{N}}\right)$ is isomorphic to $\left(\mathscr{E}_{\mathcal{N}}, \nabla_{\mathscr{E}_{N}}\right)$, we thus see that we have proven the following result.

Theorem 3.14. The canonical Frobenius lifting $\Phi_{\mathcal{N}}$ for elliptic curves (regarded parabolically) is equal to the Frobenius lifting $\Phi_{\mu}$. Moreover, the canonical indigenous bundle $\left(\mathscr{E}_{\mathcal{N}}, \nabla_{\mathscr{G}_{N}}\right)$ is the indigenous bundle constructed in Example 2 in Chapter I, $\S 2$.

Remark. In other words, what we have constructed here is just a relative version of the uniformizing $M^{\circ} \mathscr{F}^{\nabla}$-object of Definition 1.3 .

Now let $k$ be a perfect field of characteristic $p$. Then it is well-known from Serre-Tate theory that an elliptic curve $E \rightarrow \operatorname{Spec}(W(k))$ is canonical in the sense of Serre-Tate theory if and only if the point in $\alpha \in \bar{M}_{1,0}(W(k))$ that it defines is fixed by $\Phi$.u. We thus obtain that the definition of a canonical curve given in Definition 3.1 is consistent with the definition arising from Serre-Tate theory. Suppose we fix a trivialization of $\left.\mathscr{L}^{\otimes 2}\right|_{\alpha}$. Then Serre-Tate theory gives a local uniformization of $\bar{M}_{1,0}$ near this point $\alpha$ by the completion $\hat{G}_{m}$ of the multiplicative group at the identity. Relative to this uniformization, $\Phi_{\mu}$ becomes the $p^{\text {th }}$ power map on $\hat{G}_{m}$. It thus follows immediately that the canonical affine parameters that we constructed before (in the general case) correspond to the logarithm of the Serre-Tate parameter (up to multiplication by a unit of $W(k)$ ). Moreover, one sees easily that the local system $\Omega_{\mu}^{\text {et }}$ corresponding to the Frobenius lifting $\Phi_{\mathcal{M}}$ is simply $\mathscr{L}^{\otimes 2}$. Thus, a topological marking (in the sense of Definition 3.10) defines a trivialization of $\left.\mathscr{L}^{\otimes 2}\right|_{\alpha}$, and so the Serre-Tate parameter itself is a canonical multiplicative parameter in the sense of Definition 3.13. We summarize this as follows:

Theorem 3.15 Canonical liftings for elliptic curves (as defined in Definition 3.1 relative to $\left.\Phi_{N}\right)$ are the same as canonical liftings in the scnse of Serre-Tate theory. Moreover, the uniformization of Theorem 3.12 in the case of elliptic curves (regarded parabolically) is the same as the uniformization of $\mathbb{M}_{1.0}^{\text {ord }}$ given by Serre-Tate theory.

Remark. It appears that the case discussed here in Theorem 3.15, i.e., the case $g=1, r=0$, is the only case of the theory of this paper that is essentially a reformulation of a classically known theory. For instance, already in the case $g=1, r=1$, despite the fact that $M_{1,0}=M_{1,1}$ (as stacks), it is not difficult to show that $\mathcal{N}_{1,0}^{\text {ord }}$ and $\mathcal{N}_{1,1}^{\text {ord }}$ are quite different. Indeed, in general, there exist connected components of $\mathcal{N}_{1,1}^{\text {ord }}$ that are of degree $>1$ over $\mathcal{M}_{1,1}$ (cf. Proposition 3.12 of Chapter II ). This implies, in particular, that $\Phi_{\mathcal{N}}$ in the case $g=1, r=1$ is quite 
different from $\Phi_{\mathcal{N}}$ in the case $g=1, r=0$.

Finally, we observe that the term "topological marking" is apt in this case in the sense that a topological marking defines a trivialization of $\left.\mathscr{L}\right|_{\alpha}$, which is analogous in the complex case to specifying a particular pair of generators for the fundamental group of an elliptic curve.

\section{Chapter $\mathbb{I V}$ : $\mathbb{C a n o n i c a l ~ C u r v e s ~}$}

\section{§o. Introduction}

Because canonical curves (as defined in Chapter III, Definition 3.1) admit Frobenius invariant indigenous bundles, they possess a number of special arithmetic and geometric properties. In this Chapter, we study a number of these properties, foremost among which are the existence of a canonical Frobenius lifting, and the construction of a canonical $p$-divisible group. In particular, the canonical Frobenius lifting allows us to give a geometric characterization of canonical curves which may be regarded as the hyperbolic analogue of the statement in Serre-Tate theory that a lifting of an ordinary elliptic curve is canonical if and only if it admits a lifting of Frobenius. From the point of view of comparison with the complex case, this canonical Frobenius lifting may be regarded as a sort of p-adic Green's function. In the complex case, the Green's function plays a central role in the development of uniformization theory from the classical (as opposed to Bers' quasiconformal) point of view. In this context, the Green's function is essentially the logarithm of the hyperbolic distance function between two points. We shall see that the Frobenius lifting also gives us a sort of $p$-adic notion of distance. Also, we shall see that we can construct "pseudo-Hecke correspondences" which in some sense geometrically codify this notion of distance.

On the other hand, in the canonical case, we can also construct a certain Galois representation (arising from the torsion points of the canonical log $p$-divisible group) which is the $p$-adic analogue of the canonical representation in the complex case of the fundamental group into $\mathrm{PSL}_{2}(\mathbb{R})$ (defined by the covering transformations of the upper half plane). Thus, in some sense, we see that at least in the canonical case, we are able to obtain analogues of most of the fundamental objects that appear in classical complex uniformization theory.

This brings us to the final reason for wanting to study the canonical case: 
namely, the fact that the universal hyperbolically ordinary curve (over the moduli stack) is itself (essentially) a canonical curve. Thus, in Chapter V, by restricting these canonical objects over the universal curve to a given (not necessarily canonical) curve, we will be able to obtain Green's functions, canonical Galois representations, and so on for noncanonical curves, as well.

\section{§1. The Canonical Galois Representation}

In this Section, we construct a certain canonical Galois representation of the arithmetic fundamental group of a canonical curve. After studying some of the basic global properties of such representations, we then consider what happens on the ordinary locus of the curve. In particular, we construct a canonical ordinary Frobenius lifting over the ordinary locus. This allows us to apply the general theory of Chapter III, $\S 1$. We will refer to the multiplicative parameters obtained from this general theory as the Serre-Tate parameters. We will make use of the Serre-Tate parameter quite often in this Chapter.

Throughout this Section, we will work over $A=W(k)$, where $k$ is a perfect field of odd characteristic. The quotient field of $A$ will be denoted by $K$. Let $g, r$ be nonnegative integers such that $2 g-2+r \geq 1$. Also, we will deal with a fixed $\alpha \in \overline{\mathcal{N}}_{g, r}^{\text {ord }}(A)$, which corresponds to a smooth canonical curve $f^{\text {log }}: X^{\log \rightarrow}$ $S^{\log }$, where $S^{\log }$ is $\operatorname{Spec}(A)$ with the trivial log structure. Since singular canonical curves are just obtained by gluing together smooth canonical curves, we shall concentrate mainly on the smooth case.

\section{Construction and Global Properties}

Let $\left(\mathscr{E}, \nabla_{\mathscr{\Xi}}\right)$ be the canonical indigenous bundle on $X^{\text {log }}$ (whose existence is stated in Chapter III, Theorem 2.8). In fact, unless the number $r$ of marked points is even, such a vector bundle will not exist. However, one can always pass to an étale double cover of $X$ on which it will exist, and then descend. For simplicity, we will just act as though this problem does not exist, except when we state final results in Theorems, in which case our representations will be into $\mathrm{GL}^{ \pm}$(that is, the general linear group GL modulo the subgroup $\{ \pm 1\}$ ). Now, we would also like to say that the renormalized Frobenius pull-back $\mathbf{F}^{*}\left(\mathscr{E}, \nabla_{\mathscr{E}}\right)^{F}$ is isomorphic to $\left(\mathscr{E}, \nabla_{\mathscr{g}}\right)$. In general, this may only be true up to tensoring with a line bundle with connection whose square is trivial, but this may also be ignored, provided we remember that that ultimately our representations will be into $\mathrm{GL}^{ \pm}$, not GL. Let us choose an isomorphism $\Phi_{\mathscr{E}}:\left(\mathscr{E}, \nabla_{\mathscr{g}}\right) \cong \mathrm{F}^{*}(\mathscr{E}$. $\left.\nabla_{\mathscr{E}}\right)^{F}$ which is the identity on determinants. We shall call $\Phi_{\mathscr{\&}}$ the canonical Frobenius action on $\left(\mathscr{E} . \nabla_{\mathscr{E}}\right)$. 
Now let us assume that there exists a rational point $x: S \rightarrow X$ on $X$ which avoids the marked points. Let us denote by $\Pi$ the profinite group $\pi_{1}\left(X_{K}^{\text {log }}, x_{K}\right)$. Then Theorem 2.6 of [Falt] implies that

Theorem 1.1. There exists a unique dual crystalline (in the sense of [Falt], §2) representation

$$
\rho: \Pi \rightarrow \mathrm{GL}^{ \pm}(V)
$$

(where $V$ is a free $\mathbb{Z}_{p}$-module of rank two) that corresponds (under the functor $\mathbb{D}(-)$ of $[F a l t], \S 2)$ to $\left(\mathscr{E}, \nabla_{\mathscr{E}}, \bar{\Phi}_{\mathscr{E}}\right)$. Moreover, the determinant representation of $\rho$ is the cyclotomic character. We shall refer to $\rho$ as the canonical crystalline representation associated to $X^{\mathrm{log}}$.

Remark. In the complex case, a hyperbolic Riemann surface can be uniformized by the upper half plane. Then the fundamental group of the Riemann surface acts on the upper half plane via covering transformations, and so we get a representation of the fundamental group into $\operatorname{PSL}_{2}(\mathbb{R})$, which is canonically determined up to conjugation. The representation $\rho$ of Theorem 1.1 is the $p$-adic analogue of this complex representation.

Now let $\Delta=\pi_{1}\left(X_{\bar{K}}, x_{\bar{K}}\right)$ be the geometric subgroup of $\Pi$. so $\Gamma \stackrel{\text { def }}{=} \Pi / \Delta$ is the Galois group of $\bar{K}$ over $K$. Then by "the comparison theorem" (Theorem 5.3 of [Falt $]$ ), we get:

Theorem 1.2. Let $p \geq 5$. Then the group cohomology modules $H^{t}(\Delta$. Ad $(V)(1))$ (where the " $(1)$ " is a Tate twist) are zero, except when $i=1$. Let $U=H^{1}(\Delta, \mathrm{Ad}$ $(V)(1))$. Then $U$ is a crystalline $\mathbb{Z}_{p}-\Gamma$-module, which, as a $\mathbb{Z}_{p}$-module is free of rank $6(g-1)+2 r$. It corresponds under the functor $\mathbb{D}(-)$ to an MF-object (in the sense of Fontaine-Laffaille) $\left(N ; F^{\prime}(N) ; \phi^{2}\right)$ over $A$, where $N$ is a free A-module of rank $6(g-1)+2 r ; F^{\imath}(N)=0$ if $i \geq 4 ; F^{\prime}(N)=N$ if $i \leq 0 ; F^{\prime}(N)$ is naturally is isomorphic to $H^{0}\left(X,\left(\omega_{X}^{\log }\right){ }^{\otimes 2}(-D)\right)$, if $i=1,2,3$; and $N / F^{1}(N)$ is naturally isomor.

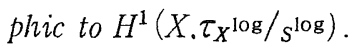

Remark. Some mathematicians have raised questions concerning that the validity of the proof in [Falt], Theorem 5.3. However, in this one-dimensional case, one can give ad hoc proofs of this result, and, moreover. (at least in the closed case, when $r=0$ ) $\mathrm{T}$. Tsuji has orally informed the author that he has obtained a different proof of Theorem 5.3 of [Falt].

One interesting fact about the canonical representation $\rho$ is that it is pos- 
sible to characterize it - as well as the canonicality of $X^{\log }$ - solely in terms of the properties of $\rho$ as a Galois representation:

Theorem 1.3. Suppose that $p \geq 5$. Let $X^{\mathrm{log}} \rightarrow \operatorname{Spec}(A)$ be any (not necessarily canonical) r-pointed smooth curve of genus $g$ over $A$. Assume that we are not in the cases $(g=0 ; r=3)$ or $(g=1 ; r=1)$. Let $\tau: \Pi \rightarrow \mathrm{GL}^{ \pm}(W)$ be any dual crys. talline representation of $\Pi=\pi_{1}\left(X_{K}^{\log }, x_{K}\right)$ on a free $\mathbb{Z}_{p}$-module $W$ of rank two such that

(1) $H_{P}^{i}(\Delta, \operatorname{Ad}(W)(1))=0$ if $i \neq 1 ; H^{1}(\Delta, \operatorname{Ad}(W)(1))$ is crystalline, and corresponds to an $M \mathscr{F}$-object $M=\left(M ; F^{\prime}(M) ; \phi^{2}\right)$ such that $F^{2}(M)=0$ if $i \geq 4 ; F^{\imath}(M)=M$ if $i \leq 0$; and $F^{\imath}(M)$ is a free A-module of rank $3(g-1)$ $+r$ if $i=1,2,3$;

(2) the Frobenius endomorphism of $\left(M / F^{1}(M)\right)_{\mathbf{F}_{p}}$ (arising from the $M F^{F-}$ object of (1)) is an isomorphism:

(3) $\operatorname{det}(\tau)$ is the cyclotomic character.

Then $X^{\log }$ is canonical, and $\tau$ is isomorphic to the representation $\rho$ of Theorem 1.1.

Proof. Since $\tau$ is asserted to be dual crystalline, it corresponds to some vector bundle with connection $(\mathscr{G}, \nabla \mathscr{G})$ on $X^{\mathrm{Hog}}$, together with a filtration $F^{i}(\mathscr{G})$ on $\mathscr{G}$. Let $i_{1}$ (respectively, $i_{2}$ ) be the largest $i$ such that $F^{i}(\mathscr{G}) \neq 0$ (respectively, $F^{\prime}(\mathscr{G})=\mathscr{G}$ ). Thus, $i_{1} \geq i_{2}$. The condition that $\operatorname{det}(\tau)$ be cyclotomic implies that $i_{1}+i_{2}=1$. If the rank of $F^{\imath_{1}}(\mathscr{G})$ is not one, then $i_{1}=i_{2}$, and $\operatorname{det}(\tau)$ could not be cyclotomic, so $F^{t_{1}}(\mathscr{G})$ must be of rank one, and $i_{1}>i_{2}$. Let $\mathscr{Q}=F^{11}(\mathscr{G})$. Thus, $\mathscr{Q}$ is a line bundle.

Let $j_{1}$ be the largest $j$ such that $F^{\prime}(M) \neq 0$. Now we claim that 2 can not be stable under $\nabla \varphi$. Indeed, if it were, then the monodromy at the marked points of $\nabla g$ on 2 , being nilpotent and one-dimensional, must be zero. Thus, the induced connection on $\mathscr{Q}$ has no poles at the marked points. But this would imply that $\operatorname{deg}(\mathscr{Q})=0$. Hence the rank over $A$ of $F^{11}(M)$ would be $\leq h^{0}\left(X, \mathscr{Q}^{\otimes 2}\right.$ $\left.\otimes \omega_{X / S}\right) \leq g<3 g-3+r$ (by Clifford's Theorem). which contradicts our hypotheses. This proves the claim. On the other hand, by Griffiths transversality, if $i_{1}-i_{2} \geq 2$, then $F^{\imath 1}(\mathscr{G})$ would have to be stable under $\nabla_{g}$. Thus, $i_{1}=i_{2}+1$, so $i_{1}$ $+i_{2}=1$ implies that $i_{1}=1$ and $i_{2}=0$.

Now $\operatorname{rank}_{A}\left(F^{3}(M)\right)=3 g-3+r \leq h^{0}\left(X, Q^{\otimes 2} \otimes \omega_{X / S}\right)$, so the line bundle $\mathscr{Q}^{\otimes 2} \otimes$ $\omega_{X / S}$ must be nonspecial, by Clifford's Theorem. It thus follows that $\operatorname{deg}\left(\mathscr{Q}^{\otimes 2}\right)$ $\geq 2 g-2+r$. Since the Kodaira-Spencer morphism for the filtration is nonzero, we cannot have $\operatorname{deg}\left(\mathscr{Q}^{\otimes 2}\right)>2 g-2+r$. Thus, we see that $(\mathscr{G}, \nabla \xi)$ must be indige nous. Since it is also carried to itself by the renormalized Frobenius, it follows 
from Chapter III, Corollary 3.4 , that $X^{\log }$ is canonical, and that $\left(\mathscr{G}, \nabla_{\mathscr{G}}\right)$ must be the canonical indigenous bundle of Theorem 1.1.

Remark. For the reader who is interested in handling the cases $g=0 ; r=3$ and $g=1 ; r=1$, as well, we remark that by considering conditions (similar to those imposed on $\left.H^{2}(\Delta, \operatorname{Ad}(W)(1))\right)$ on higher symmetric powers of $W$, one can characterize the canonical representations in these cases as well solely in terms of their properties as Galois representations.

Remark. Really, the substantive missing element here is that it is not clear to the author how to characterize the property of being "dual crystalline" solely in terms of properties of the representation relative to the triple $(\mathbb{I} ; \Delta \subseteq \mathbb{I}$; $\Pi / \Delta \cong \mathrm{Gal}(\bar{K} / K))$. Thus, ultimately, a knowledge of the curve $X^{\log }$ is always present in the background of this "Galois representation-theoretic" characterization of the canonical representation. For instance, if the property of being "dual crystalline" were known to depend only on the triple $(\Pi ; \Delta \subseteq \mathbb{\Pi}: \mathbb{\Pi} / \Delta \cong$ $\mathrm{Gal}(\bar{K} / K)$ ), then one could obtain the result that whether or not a curve is canonical depends only on that triple.

In the following, we return to the assumption that $X^{\text {log }}$ is canonical.

\section{The Horizontal Section over the Ordinary Locus}

We maintain the notation of the previous subsection. Let $X^{\text {ord }}$ be the $p$-adic formal scheme which is the open sub-formal scheme of $X$ given by the complement of the supersingular divisor (Chapter II. Proposition 2.6). Let us endow $X^{\text {ord }}$ with the log structure induced by $X^{\text {log }}$, and call the resulting log formal scheme $\left(X^{\mathrm{lug}}\right)^{\text {ord }}$. We shall refer to $\left(X^{\mathrm{log}}\right)^{\text {ord }}$ as the ordinary locus of $X^{\mathrm{log}}$. The purpose of this subsection is to prove and interpret the following result:

Theorem 1.4. There exists a unique subbundle $\left.\mathcal{T}^{\frac{1}{2}} \subseteq \mathscr{E}\right|_{X^{\text {ord }}}$ of rank one with the following properties:

(1) $\mathscr{T}^{\frac{1}{2}}$ is horizontal, and moreover, for any $n$, the reduced line bundle $\mathscr{T}_{\frac{\mathbf{Z} / p^{n \mathbf{Z}}}{\frac{1}{2}}}$ has a nonempty subsheaf (in the category of sets) consisting of horizontal sections that generate $\mathcal{T}_{\frac{\mathrm{Z} / p n \mathrm{Z}}{2}}^{\frac{1}{2}}$ as an $\mathcal{O}_{X}$ ord-module;

(2) $\mathscr{T}^{\frac{1}{2}}$ is taken to itself by $\Phi_{8}$.

Finally, $\left(\mathscr{T}^{\frac{1}{2}}\right)^{\otimes 2}$ is naturally isomorphic to $\tau_{X}^{\log } /\left.S^{\log }\right|_{X}$ ord.

Proof. Let us prove that there exists a unique $\Phi_{\mathscr{E}}$-invariant horizontal sub- 
bundle $\left.\mathscr{T}^{\frac{1}{2}} \subseteq \mathscr{E}\right|_{X}$ ord with horizontal generating sections. We prove this by induction on $n$, where the $n^{t h}$ step is the construction of such a $\mathscr{T}^{\frac{1}{2}}$ modulo $p^{n}$. For $n=1$, recall that (up to tensoring with a line bundle) $\mathscr{E}_{\mathrm{p}}$ is an FL-bundle (Chapter II, Proposition 2.5). Then under the correspondence of that Proposition, we take our subbundle $\mathscr{T}_{\mathrm{F}_{p}}^{\frac{1}{2}}$ to be the subbundle of $\mathscr{E}$ corresponding to the subbundle that we called " $\mathscr{T}$ " in our discussion of FL-bundles in Chapter II, $\S 1$. This subbundle is clearly horizontal, and has local generating sections that are horizontal. In this case, uniqueness follows from the fact that the $p$-curvature is nonzero.

Now we assume that $n \geq 2$, and that the result is known for $n-1$. Let $U^{\text {log }}$ $=\left(X^{\log }\right)^{\text {ord }}$. and let $\Phi^{\log }: U^{\log } \rightarrow\left(U^{\log }\right)^{F}$ be a Frobenius lifting. Let us consider the quotient $\mathscr{Q}$ (respectively, $\mathscr{P}$ ) of $\mathscr{E} \mathrm{Z} / p^{n} \mathrm{Z}$ by $p^{n-1} \cdot F^{1}(\mathscr{E})$ (respectively, $p^{n-1} \cdot$ $\mathscr{E})$. Thus, $\mathscr{P}$ is a quotient of $\mathscr{Q}$, and $\mathscr{P}=\mathscr{E} \mathrm{z} / p^{n-1 \mathrm{Z}}$. Let $\mathscr{T}^{\prime \prime} \subseteq \mathscr{P}$ be the subbundle given us by the induction hypothesis. Let $\mathscr{T}^{\prime} \subseteq \mathcal{Q}$ be the inverse image of $\mathscr{T}^{\prime \prime} \subseteq$ $\mathscr{P}$ via the surjection $\mathcal{Q} \rightarrow \mathscr{P}$. Then $\Phi^{*}\left(\mathscr{T}^{\prime}\right)^{F} \subseteq \Phi^{*} \mathscr{Q}^{F}$ defines a subbundle $\mathcal{J}_{\mathbf{Z} / \not n \mathbf{Z}}^{\frac{1}{2}}$ of $\mathbb{F}^{*}(\mathscr{E})_{\mathbf{Z} / p n \mathbf{Z}}^{\mathrm{F}}$. It follows from the definition of $\mathscr{T}_{\mathbf{F}_{p}}^{\frac{1}{2}}$ and the fact that we are on the ordinary locus that $\mathscr{T}_{\mathbf{Z} / p^{n} \mathbf{Z}}^{\frac{1}{2}}$ is flat over $\mathbb{Z} / p^{n} \mathbb{Z}$. The existence of local horizontal generating sections for $\mathscr{T}_{\mathbf{Z} / p n \mathbf{Z}}^{\frac{1}{2}}$ follows by taking such a section of $\mathscr{T}^{\prime \prime}$, lifting it to $\mathscr{T}^{\prime}$, and then pulling back this lifted section of $\mathscr{J}^{\prime}$ to a section of $\mathscr{T}_{\mathrm{Z} / p^{n} \mathbf{Z}}^{\frac{1}{2}}$ via $\Phi^{*}$. That the connection vanishes on this section follows from the definitions, plus the fact that pulling back by $\Phi$ adds an extra factor of $p$. Since $\mathscr{T}^{\prime \prime}$ is $\Phi_{\mathscr{E}}$-invariant, it follows that $\mathscr{T}_{\mathbf{Z} / p n \mathbb{Z}}^{\frac{1}{2}} \otimes \mathbb{Z} / p^{n-1} \mathbb{Z}=\mathscr{T}^{\prime \prime}$. Thus, by the construction of $\mathscr{T}_{\mathrm{Z} / p n \mathrm{Z}}^{\frac{1}{2}}$, it is clear that $\mathscr{J}_{\mathrm{Z} / p n \mathrm{Z}}^{\frac{1}{2}}$ is $\Phi_{\mathscr{g}}$-invariant. since pulling back any lifting of $\mathscr{T}^{\prime \prime}$ by $\Phi_{g}$ will give $\mathscr{T}_{\mathbf{Z}^{\prime} \not n \mathbb{Z}}^{\frac{1}{2}}$. Also, this same observation (coupled with the induction hypothesis) proves uniqueness. This completes the proof of the induction step. The last statement about $\left(\mathscr{T}^{\frac{1}{2}}\right)^{\otimes 2}$ follows from considering the splitting of the Hodge filtration that $\mathscr{T}^{\frac{1}{2}}$ defines.

Now suppose that our basepoint $x: S \rightarrow X$ maps into the ordinary locus $X^{\text {ord }}$. Let $\Pi^{\text {ord }}=\pi_{1}\left(\left(X^{\text {log }}\right)_{K}^{\text {ord }}, \pi_{K}\right)$. Thus, we have a natural morphism:

$$
\Pi^{\text {ord }} \rightarrow \Pi
$$

Let us denote the restriction of $\rho$ to $\Pi^{\text {ord }}$ via this natural morphism by

$$
\rho^{\text {ord }}: \Pi^{\text {ord }} \rightarrow \mathrm{GL}^{ \pm}\left(V^{\text {ord }}\right)
$$


Then if we apply the theory of [Falt], $\S 2$ to interpret Theorem 1.4, we see that

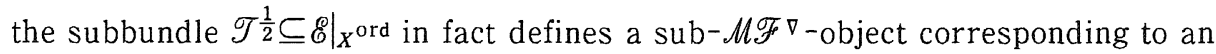
étale representation $\left(\rho^{\text {ord }}\right)$ et $: \Pi^{\text {ord }} \rightarrow \mathrm{GL}^{ \pm}\left(V_{\text {et }}\right)$ of $\Pi^{\text {ord }}$, for some rank one free $\mathbb{Z}_{p}$-quotient module $V^{\text {ord }} \rightarrow V_{\text {et. }}$. Here, by "étale," we mean that the kernel of

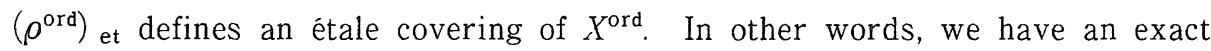
sequence of ("up to $\{ \pm 1\}$ ") representations of $\mathbb{I}^{\text {ord: }}$

$$
0 \rightarrow V_{\mathrm{et}}^{\vee}(1) \rightarrow V^{\text {ord }} \rightarrow V_{\mathrm{et}} \rightarrow 0
$$

where the " 1 " in parentheses is a Tate twist. We state this as a Corollary:

Corollary 1.5. The restriction $\rho^{\text {ord }}$ of $\rho$ to $\mathbb{\Pi}^{\text {ord }}$ defines an ("up to $\{ \pm 1\}$ ") module $V^{\text {ord }}$ of $\Pi^{\text {ord }}$, which fits into an exact sequence:

$$
0 \rightarrow V_{\mathrm{et}}^{\vee}(1) \rightarrow V^{\text {ord }} \rightarrow V_{\text {et }} \rightarrow 0
$$

where $V_{\mathrm{et}}$ is étale and of rank one over $\mathbb{Z}_{p}$.

\section{The Canonical Frobenius Lifting over the Ordinary Locus}

In this subsection, we construct the generalized analogue (for an arbitrary canonical $X^{\mathrm{log}}$ ) of the $p$-adic endomorphism of the ordinary locus of the moduli stack of elliptic curves obtained by sending an elliptic curve with ordinary reduction to its quotient modulo its unique subgroup scheme which is étale locally isomorphic to $\mu_{p}$. In many respects, the construction is similar to (although not literally a logical consequence of) the construction of the Frobenius lifting on $\overline{\mathcal{N}}_{g, r}^{\text {ord }}$ constructed in Chapter III. $\S 2$.

Consider the canonical indigenous bundle $\left(\mathscr{E} . \nabla_{\mathscr{E}}\right.$ ) (of Theorem 1.1) on the canonical curve $X^{\log } \rightarrow S^{\log }$. By Chapter II, Proposition 2.5, $\left(\mathscr{E}, \nabla_{\mathscr{E}}\right) \mathbf{F}_{p}$ corresponds to an FL-bundle

$$
0 \rightarrow \mathscr{T}_{\mathrm{F}_{p}} \rightarrow \mathscr{F} \rightarrow \mathscr{O}_{X_{F_{p}}} \rightarrow 0
$$

on $X_{F_{p}}^{\log }$. By the material directly preceding Chapter II, Proposition 1.2, splittings of this exact sequence correspond to Frobenius liftings on $X_{\mathbf{Z} / p 2 Z}^{\log _{p}}$. Now, over the ordinary locus of $X^{\mathrm{log}}$, the Hodge filtration defines such a splitting. Let us denote the resulting Frobenius lifting on the ordinary locus by

$$
\bar{\Phi}_{2}:\left(X^{\mathrm{log}}\right) \underset{\mathrm{Z} / p^{2} \mathbf{Z}}{\stackrel{\mathrm{rd}}{\longrightarrow}} \rightarrow\left(\left(X^{\log }\right)^{\text {ord }}\right)_{\mathbf{Z} / p^{2} \mathbf{Z}}^{F}
$$


Let us denote by $\widetilde{\mathscr{E}}$ the vector bundle which is the inductive limit of the following diagram:

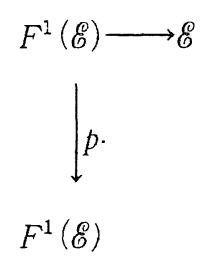

where the horizontal arrow is the natural inclusion. Note that $\widetilde{\mathscr{E}}_{\mathbf{Z} / p^{n \mathbf{Z}}}$ depends only on $\mathscr{E} \mathbf{z} / \not n \mathbf{Z}$. By the definition of the renormalized Frobenius pull-back, $\Phi_{2}^{*} \widetilde{E}_{\mathrm{Z} / p 2 \mathrm{Z}}^{F}$ is naturally isomorphic to $\mathscr{E} \mathrm{Z} / \mathrm{p}^{2 \mathrm{Z}}$. We shall identify these two sheaves in the following discussion. On the other hand, by considering the object in the upper right-hand corner of the diagram defining $\widetilde{\mathscr{E}}$, we obtain a morphism

$$
\Phi_{2}^{*} \mathscr{E} \mathrm{Z}_{p 2 \mathrm{Z}} \rightarrow \mathscr{E}_{\mathrm{Z} / p^{2} \mathrm{Z}}
$$

whose restriction to $\Phi_{2}^{*} F^{1}(\mathscr{E}){ }_{\mathrm{Z} / p^{2} \mathbf{Z}}^{F}$ vanishes on $\left.p \cdot \Phi_{2}^{*} F^{1}(\mathscr{E})\right)_{\mathrm{Z} / p^{2} \mathbf{Z}}^{F}$ and maps $\Phi_{2}^{*} F^{1}\langle\mathscr{E})_{\mathbf{Z} / p^{2} \mathbf{Z}}^{F}$ into $p \cdot F^{1}(\mathscr{E})_{\mathbf{Z} / p^{2} \mathbf{Z}}$ (by the definition of the correspondence between Frobenius liftings and splittings of the FL-bundle $\mathscr{F})$.

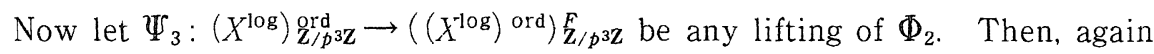
from the definition of the renormalized Frobenius pull-back, we obtain a morphism

$$
\Psi_{3}^{* \mathscr{E} \mathrm{Z} / p^{3 \mathrm{Z}}} \rightarrow \stackrel{\mathscr{E} \mathrm{Z} / p^{3} \mathrm{Z}}{ }
$$

which vanishes on $p^{2} \cdot \Psi_{3}^{*} F^{1}(\mathscr{E}){ }_{\mathrm{Z} / p^{3} \mathbf{Z}}^{F}$. However, if $\Psi_{3}$ is an arbitrary lifting of $\Phi_{2}$, then we don't know that $\Psi_{3}^{*} F^{1}(\mathscr{E}) \mathbb{Z}_{/ p^{3} \mathrm{Z}}$ is mapped into $F^{1}(\mathscr{E}) \mathbf{Z}_{/ p 3} \mathrm{Z}$.

Now suppose that we modify $\Psi_{3}$ by a section $\delta \in \Gamma\left(X^{\text {ord }}, \mathscr{T}_{\mathbf{F}_{p}}\right)$. Let $H_{\mathcal{T}}$ : $\left.\mathscr{T}_{\mathrm{F}_{p}} \rightarrow\left(\tau_{X^{108} / \mathcal{S}^{108}}\right)_{\mathrm{F}_{p}}\right|_{X^{\text {ord }}}$ be the isomorphism defined by projecting to the Hodge filtration. Then the subsheaf of $\mathscr{E} \mathbf{Z} / p^{3} \mathbf{Z}$ given by the image of $\left.F^{1}(\mathscr{E})\right)_{\mathbf{Z} / p^{3} \mathbf{Z}}^{F}$ under $\Psi_{3}$ $+\delta$ differs from the corresponding image subsheaf under $\Psi_{3}$ by the amount $H$ g $(\delta) \in \Gamma\left(X^{\text {ord }},\left(\tau_{X^{108} / S^{108}}\right) \mathbf{F}_{p}\right)$. Indeed, this follows from the definitions, plus the fact that the Kodaira-Spencer morphism for $\mathscr{E}$ is the identity. Since $H_{\mathscr{T}}$ is an isomorphism, it thus follows that there exists a unique Frobenius lifting

$$
\Phi_{3}:\left(X^{\mathrm{log}}\right)_{\mathrm{Z} / p^{3 Z}}^{\mathrm{ord}} \rightarrow\left(\left(X^{\mathrm{log}}\right)^{\text {ord }}\right)_{\mathrm{Z} / p^{3} \mathrm{Z}}^{F}
$$

that lifts $\Phi_{2}$ such that $\Phi_{3}^{*}$ maps $F^{1}(\mathscr{E}) \mathbb{Z}_{/ p^{3} Z}$ into $F^{1}(\mathscr{E})$ 
Clearly, we may repeat this procedure modulo $p^{n}$ for arbitrary $n \geq 3$, so as to obtain a unique

$$
\Phi_{X}^{\log }:\left(X^{\log }\right)^{\text {ord }} \rightarrow\left(\left(X^{\log }\right)^{\text {ord }}\right)^{F}
$$

such that under the natural morphism

$$
\Phi_{X}^{* \mathscr{E} F} \rightarrow \mathscr{E}
$$

the Hodge filtration is preserved. Note, moreover, that it follows from the fact that the Kodaira-Spencer morphism at the Hodge section is an isomorphism plus the interpretation of the FL-bundle $\mathscr{F}$ in terms of Frobenius lifting that this Frobenius lifting $\Phi_{X}^{\text {log }}$ is ordinary in the sense of Chapter III, Definition 1.1. In summary, we have proven the following result:

Theorem 1.6 . Let $X^{\mathrm{log}}$ be a canonical curve: $\left(\mathscr{E}, \nabla_{\mathscr{E}}\right)$ the canonical indigenous bundle on $X^{\mathrm{log}}$. Then there exists a unique ordinary Frobenius lifting (called canonical)

$$
\bar{\Phi}_{X}^{\log }:\left(X^{\log }\right)^{\text {ord }} \rightarrow\left(\left(X^{\log }\right)^{\text {ord }}\right)^{F}
$$

over the ordinary locus that preserves the Hodge filtration.

In particular, we can apply the theory of Chapter III, $\S 1$, to the Frobenius lifting $\Phi_{X}^{\text {log }}$. Note that it follows immediately from the definitions that the $M \mathscr{F}^{\nabla}$-object $\left.\left(\mathscr{E}, F^{1}(\mathscr{E}), \nabla_{\mathscr{E}}, \Phi_{\mathscr{E}}\right)\right|_{\left(X^{108}\right) \text { ord }}$ is precisely the uniformizing $M \mathscr{F}^{\nabla}$-object associated to $\Phi_{X}^{\log }$ (as in Chapter III, Definition 1.3). Let us write

$$
\mathscr{T}=\left(\mathscr{T}^{\frac{1}{2}}\right)^{\otimes 2}
$$

Thus, $\nabla_{\mathscr{f}}$ (respectively, $\bar{\Phi}_{g}$ ) induces a natural connection (respectively, Frobenius action) on $\mathscr{T}$, which defines the canonical tangential local system of Chapter III, Definition 1.2. Since $\mathscr{T}$ is a line bundle, it is the same to give (over an étale covering of $X^{\text {ord }}$ ) a generating Frobenius invariant section of it, or a generating Frobenius invariant section of its dual. Thus, (just as in Chapter III, Definition 1.11) if $\theta$ is such a section of $\mathscr{T}$, then $\theta$ defines, at every $z \in X^{\text {ord }}$ $(A)$ that avoids the marked points, a unique multiplicative parameter

$$
q_{\theta} \in R_{z}^{\times}
$$


(where $R_{z}$ is the completion of $X^{\text {ord }}$ at $z$ ). If the residue of $\theta$ is equal to one at a marked point $z \in X^{\text {ord }}(A)$, then we get a multiplicative parameter

$$
q \in R_{z}
$$

(with valuation one at the divisor $\operatorname{Im}(z)$ ) which is unique up to multiplication by a Teichmüller representative $\left[k^{\times}\right]$.

Definition 1.7. We shall call $q$ (respectively, $q_{\theta}$ ) the Serre-Tate parameter (respectively, relative to $\theta$ ) at $z$.

Note, in particular, that by the theory of Chapter III, $\S 1, \Phi_{X}^{-1}$ maps $q$ (respectively, $q_{\theta}$ ) to $q^{p}$ (respectively, $q_{\theta}^{p}$ ).

Remark. In some sense, it would be more aesthetically pleasing if one could obtain the Frobenius lifting of Theorem 1.6 in the following way. We consider the universal curve $\mathscr{C} \rightarrow \overline{\mathcal{N}}_{g, r}^{\text {ord }}$. Then $\mathscr{C}$ parametrizes $(r+1)$-pointed stable curves of genus $g$, so we have a Frobenius lifting on some stack which is étale over $\mathscr{C}$. If we could prove that this Frobenius lifting is compatible with the canonical Frobenius lifting on $\overline{\mathcal{N}}_{g, r}^{\text {ord }}$, then we could obtain a canonical Frobenius lifting on $\mathscr{C}$ (or at least some stack étale over $\mathscr{C}$ ) simply by using the canonical Frobenius on $\overline{\mathcal{N}}_{q, r+1}^{\text {ord }}$.

The problem with this approach is that despite the fact that the canonical modular Frobenius liftings of Chapter III do have many interesting functorial relations (i.e., relative to restriction to the boundary and log admissible coverings), in general, the sort of compatibility of Frobenius liftings necessary to make the above sketch of a proof work - namely, compatibility with "forgetting a marked point" - simply does not hold. Indeed, one can already see this in the case of the morphism

$$
\bar{M}_{1,1} \rightarrow \bar{M}_{1,0}
$$

which is the identity on the underlying stacks, but which we think of as assigning to a one-pointed curve of genus one the underlying elliptic curve. Here, the canonical Frobenius on $\overline{\mathcal{N}}_{1,1}^{\text {ord }}$ cannot be compatible with the canonical Frobenius on $\overline{\mathcal{N}}_{1,0}^{\text {ord }}$ for the following reason. Since $\overline{\mathcal{N}}_{1,0}^{\text {ord }} \hookrightarrow \overline{\mathcal{N}}_{1,0}$ is an open immersion, it would follow that the canonical Frobenius on $\overline{\mathcal{N}}_{1,1}^{\text {ord }}$ would descend to an open formal subscheme of $\bar{M}_{1,1}$. But this would mean that even if a one-pointed curve of genus one in characteristic $p$ belongs to several distinct quasiconformal 
equivalence classes (a phenomenon which by Chapter II, Proposition 3.13, does occur), the canonical liftings of that curve would be the same for all quasiconformal equivalence classes. But this would mean that we have several different ordinary indigenous bundles on a single hyperbolic curve, all of which are Frobenius invariant. By Chapter III, Lemma 2.6, this is absurd.

\section{§2. The Canonical $\log p$-Divisible Group}

Although the existence of the canonical Galois representation of $\S 1$ is, in and of itself, of some interest, one technical drawback that it has is that it is difficult to relate the properties of the Galois representation or the characteris-

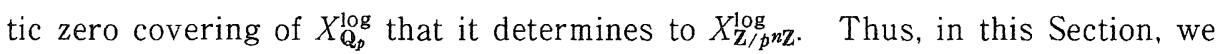
shall construct a $\log p$-divisible group on $X^{\text {log }}$ which gives us back the canonical Galois representation (by looking at the Galois action on torsion points), but which has the advantage that one can study and understand its reductions modulo $p^{n}$ in a similar fashion to the elliptic modular case (which is studied in $[\mathrm{KM}])$.

\section{Log $p$-divisible Groups at Infinity}

We maintain the notation of the previous Section (although $k$ need not be algebraically closed, just perfect). For basic facts about log schemes, we refer to [Kato] and [Kato 2]. In [Kato 2], certain finite, log flat group objects over the compactified moduli stack of elliptic curves are introduced which are supposed to be the analogue at infinity of the usual finite, flat group schemes that one gets from elliptic curves by considering the kernel of multiplication by a power of $p$. Since we will use such objects (as well as the $p$-divisible group objects obtained by taking direct limits thereof) later in this Section, we take the time out in the present subsection to review explicitly the construction of these finite. log flat group objects.

Let $R=A[[q]]$ be a complete local ring which is formally smooth of dimension one over $A$. If one inverts $q$, then by taking the $\left(p^{n}\right)^{\text {th }}$ root of $q$, one obtains an extension of finite flat group schemes

$$
0 \rightarrow \mathbb{Z} / p^{n} \mathbb{Z}(1) \rightarrow G_{n} \rightarrow \mathbb{Z} / p^{n} \mathbb{Z} \rightarrow 0
$$

over $R[1 / q]$. Because $q$ is not a unit in $R$, it is impossible to extend this extension of finite flat group schemes over $R[1 / q]$ to an extension of finite flat group schemes over $R$. Our goal in this subsection, however, is to exhibit a natural extension of the above exact sequence to an exact sequence defined over $R$ by 
working with group objects in the category of finite, log flat log schemes over $\operatorname{Spec}(R)^{\log }$. (In this subsection, we will regard $\operatorname{Spec}(R)$ as endowed with the log structure arising from the divisor defined by q.)

For nonnegative integers $a, b$, let $M_{a, b}$ be the monoid given by taking the quotient of $\mathbf{N}^{2}$ (where $\mathbf{N}$ is the monoid of nonnegative integers) by the equivalence relation generated by $\left(p^{a}, 0\right) \sim(0, b)$. Let $e_{1} \in M_{a, b}$ (respectively, $e_{2} \in M_{a, b}$ ) be the image of $(1,0)$ (respectively, $(0,1)$ in $M_{a, b}$. Then it follows from the theory of [Kato 2] (especially. §4.1, 5.1) that we can construct the desired extension

$$
0 \rightarrow \mathbb{Z} / p^{n} \mathbb{Z}(1) \rightarrow \bar{G}_{n}^{\log } \rightarrow \mathbb{Z} / p^{n} \mathbb{Z} \rightarrow 0
$$

as follows: For $j \in\left\{0, \cdots, p^{n}-1\right\}$, consider the scheme $G_{n, j}^{\prime}$ given by $R[x] /\left(x^{p^{n}}-q^{3}\right)$, with the $\log$ structure given by the chart ([Kato], §2) $M_{n, 3}$ with $e_{1} \mapsto x ; e_{2} \mapsto q$. Denote the resulting log scheme by $\left(G^{\prime}\right)_{n, j}^{\log }$. Let $G_{n, j}^{\log }$ be the universal valuative log space ([Kato 2], §1.3.1) (which. in this case, will still be a log scheme) associated to $\left(G^{\prime}\right)_{n, j}^{\log }$. Let $\bar{G}_{n}^{\log }$ be the union of the $G_{n, j}^{\log }$. Note that when we in vert $q, \bar{G}_{n}^{\text {log }}$ becomes $G_{n}$. Endow $\bar{G}_{n}^{\text {log }}$ with the unique structure of group object that extends the group structure on $G_{n}$. Then $\bar{G}_{n}^{\text {log }}$ is a group object in the category of finite, log flat log schemes over $\operatorname{Spec}(R)^{\log }$. and it fits into an exact sequence as above.

As well allow $n$ to vary, we get morphisms $\bar{G}_{n}^{\log } \rightarrow \bar{G}_{n+1}^{\log }$. Thus, we obtain an ind-group object $\bar{G}$ over $\operatorname{Spec}(R)^{\log }$.

Definition 2.1. We shall refer to $\bar{G}$ as the $\log p$-divisible group over Spec $(R)^{\log }$ obtained by taking $p^{\text {th }}$ power roots of $q \in R$.

Finally, we remark that, although what we are doing here is, in some sense, just "trivial general nonsense," its utility lies in the fact that by using it, we can obtain $p$-adic finite coverings of $X^{\log }$ that are defined over all of $X^{\log }$, thus allowing us to algebrize.

\section{Construction of the Canonical $\log p$-Divisible Group}

We now turn to the construction of the canonical log $p$-divisible group on $X^{\mathrm{log}}$. Consider the $\mathcal{M} \mathscr{F}^{\nabla}$-object $\left(\mathscr{E}, \nabla_{\mathscr{g}}, \Phi_{\mathscr{g}}\right)$, defined by the canonical indigenous bundle. Let $n \geq 1$. Let $U \subseteq X$ be the open $p$-adic subscheme defined by removing the marked points. Then the reduction modulo $p^{n}$ of $\left.\left(\mathscr{E}, \nabla_{\mathscr{E}}, \Phi_{\mathscr{E}}\right)\right|_{U}$ defines, by 
[Falt], Theorem 7.1, a finite, flat group scheme (annihilated by $p^{n}$ ), which we denote by $\left.G_{n}\right|_{U} \rightarrow U$. On the other hand, let $R$ be the complete local ring at any one of the marked points. Then $\left(\mathscr{E}, \nabla_{\mathscr{E}}, \Phi_{\mathscr{E}}\right)$ defines a Serre-Tate parameter (as in Definition 1.7) $q \in R /\left[k^{\times}\right]$. Let $\tilde{q} \in R$ be any representative of $q$. Then $R=$ $A[[\tilde{q}]]$. Let $\left.G_{n}^{\log }\right|_{R}$ be the log scheme constructed in the previous subsection by taking a $\left(p^{n}\right)^{t h}$-root of $q$. Observe that different choices of $\tilde{q}$ give us naturally isomorphic $\left.G_{n}^{\log }\right|_{R}$ 's. Also, note that if we invert $\tilde{q}$, then $\left.G_{n}^{\log }\right|_{R}$ becomes $\left.\left(\left.G_{n}\right|_{U}\right)\right|_{R}$. Thus, we see that $\left.G_{n}\right|_{U}$ and the various $\left.G_{n}^{\log }\right|_{R}$ at the marked points glue together naturally to form a finite, $\log$ flat group object $G_{n}^{\log } \rightarrow X^{\log }$, which a priori is just $p$-adic, but may be algebrized since $X$ is proper over $A$. Also, as $n$ varies, we obtain natural morphisms

$$
\cdots \rightarrow G_{n}^{\log } \rightarrow G_{n+1}^{\log } \rightarrow \cdots
$$

which thus form an inductive system of group objects.

Definition 2.2. We shall call this inductive system of group objects the canonical $\log p$-divisible group on $X^{\log }$.

Remark. As usual, strictly speaking we really have only defined a "group up to $\{ \pm\}$." That is, we really only have a group object over (perhaps) a finite étale covering of $X$ of degree 4, plus descent data (satisfying the cocycle condition $u p$ to $\{ \pm 1\}$ ) down to the original $X$. We could, of course, develop the general nonsense of such "groups up to $\{ \pm 1\}$," but we choose not to, since it seems to serve no real purpose.

If we invert $p$, then this $\log p$-divisible group $G^{\log }$ on $X^{\log }$ defines a local system on the étale site $\left(X_{\mathbf{Q}_{p}}^{\mathrm{log}}\right)_{\text {et }}$ in free $\mathbb{Z}_{p}$-modules of rank two. Thus, we get a Galois representation on the Tate module $T$ of characteristic zero torsion points of $G^{\log \text { : }}$

$$
\rho_{G^{\log }}: \Pi \rightarrow \mathrm{GL}^{ \pm}(T)
$$

Then we have the following result (which is immediate from the theory of [Falt], especially the construction in the proof of Theorem 7.1):

Proposition 2.3. The representation $\rho_{G^{108}}$ is isomorphic to the canonical Galois representation $\rho$ of Theorem 1.1 . 


\section{Review of the Theory of [Katz-Mazur]}

In this subsection, we apply to the $\log p$-divisible group $G^{\log }$ the theory of $[\mathrm{KM}]$, which is exposed in $[\mathrm{KM}]$ solely in the case of the canonical log $p$-divisible group on the compactified moduli stack of elliptic of curves, but whose proofs go through without change for the canonical $\log p$-divisible group $G^{\mathrm{log}}$ on any canonical curve $X^{\mathrm{log}}$.

First of all, because $G^{\log }$ is a logarithmic $p$-divisible group, it follows from [Mess], Chapter II, Theorem 3.3.13, that if we consider the formal neighborhood of the identity section $\epsilon: X \rightarrow G$, we obtain a formally smooth formal scheme $\widehat{G}(\epsilon)$ over $X$, which is easily seen to have relative dimension 1 over $X$. We would like to use this observation to apply the theory of $[\mathrm{KM}]$, Chapter 1, on "A-generators" and "A-structures" to $G^{\log }$. The theory there goes through just as in the modular case since the only technical assumption needed on the finite, flat (logarithmic) group schemes whose $A$-generators we wish to parametrize is that they be closed subschemes of some smooth one-dimensional scheme. However, looking at the proofs of $[\mathrm{KM}]$, one sees that in fact, in suffices to have the finite, flat $(\log )$ group schemes be closed subschemes of a formally smooth formal scheme (such as $\widehat{G}(\epsilon)$ ) of relative dimension one. Thus, we can define various moduli problems, just as in $[\mathrm{KM}]$. Chapter 3 , by means of various structures:

(1) a $\Gamma(n)$-structure, which consists of giving a Drinfeld basis for $G_{n}^{\text {log. }}$

(2) a $\Gamma_{1}(n)$-structure, which consists of giving a point "of exact order $p^{n}$ " in $G_{n}^{\text {log; }}$

(3) a $\Gamma_{0}(n)$-structure, which consists of giving an isogeny $G^{\text {log }} \rightarrow H^{\text {log }}$ (where $H^{\log }$ is also a $\log p$-divisible group) whose kernel is cyclic of order $p^{n}$.

Moreover, just as in $[\mathrm{KM}]$. one proves that these various moduli problems are representable by schemes $X(n) ; X_{1}(n) ; X_{0}(n)$ that are finite over $X$. Finally, all of these schemes $X(n) ; X_{1}(n)$; and $X_{0}(n)$ are, in fact, regular. Indeed, away from the marked points, the proofs of regularity in [KM], Chapters 5 and 6 , boil down to general nonsense plus two technical results (Proposition 5.3.4 and Theorem 6.1.1). Since these technical results are proven, respectively, for arbitrary formal groups and arbitrary finite group schemes, it is immediate that the regularity proofs of $[\mathrm{KM}]$ in the modular case go through without change for $X(n): X_{1}(n)$; and $X_{0}(n)$. At the marked points, the combinatorial descriptions of the situation at the cusps in $[\mathrm{KM}]$. Chapter 10, go through without change 
for the above moduli problems. We thus obtain the following Theorem:

Theorem 2.4. The schemes $X(n) ; X_{1}(n)$; and $X_{0}(n)$ that represent the moduli problems listed above are all regular, and hence equal to the normalizations of $X$ in the finite coverings of $X_{K}$ defined by the appropriate composites of $\rho: \Pi \rightarrow \mathrm{GL}^{ \pm}(V)$ with finite quotients $\mathrm{GL}^{ \pm}(V) \rightarrow G$, just as in the classical modular case.

Fix a positive integer $n$. We shall also need to analyze $X_{0}(n)$ modulo $p$, in a fashion similar to what is done in the modular case in [KM], Chapter 13. Let us (for the rest of the Section) denote $X_{0}(n)$ by $Y$, and let us use a subscript $m$ on $X, Y$, etc., to denote reduction modulo $p^{m+1}$. Let us denote by $\Phi_{A}: A \rightarrow A, \Phi_{k}$ : $k \rightarrow k$ the respective absolute Frobenius morphisms, and by a superscript $F^{m}$ the result of base-changing an object by the $m^{\text {th }}$ power of Frobenius, and by $\Phi_{X_{0}}$ : $X_{0} \rightarrow X_{0}^{F}$ the relative Frobenius of $X_{0}$. Essentially, the description of $Y_{0}=Y \otimes_{\mathrm{Z}_{p}}$ $\mathbb{F}_{p}$ given in $[\mathrm{KM}]$, Chapter 13 , goes through in our situation here, but we need to do things with a little bit more care, since [KM] often falls back on the "crutch" of using the modular interpretation of their " $X$," which we lack in this more general situation.

For each ordered pair of nonnegative integers $(a, b)$ such that $a+b=n$, we would like to define a $k$-scheme $X_{0}(a, b)$ of " $(a, b)$-cyclic isogenies" together with a $k$-morphism $\iota_{(a, b), 0}: X_{0}(a, b) \rightarrow Y_{0}$. We do this as follows. If $a, b \geq 1$, then we let $X_{0}(a, b)$ be the schematic inverse of image of $\operatorname{Inf}^{p-1}(\Delta)$ (the $(p-1)^{\text {st }}$ infinitesimal neighborhood of the diagonal) via

$$
\Phi_{X_{0}}^{a-1} \times \Phi_{X_{0}}^{b-1}: X_{0} \times X_{0}^{F^{a-b}} \rightarrow X_{0}^{F^{a-1}} \times X_{0}^{F^{a-1}}
$$

If $a$ or $b$ is zero, then we let $X_{0}(a, b)$ be the schematic inverse image of the diagonal $\Delta$ via

$$
\Phi_{X_{0}}^{a} \times \Phi_{X_{0}}^{b}: X_{0} \times X_{0}^{F^{a-b}} \rightarrow X_{0}^{F^{a}} \times X_{0}^{F^{a}}
$$

Observe that in either case, $\left(X_{0}(a, b)\right)_{\text {red }}$ is smooth over $k ; \Phi_{X_{0}}^{a} \times \Phi_{X_{0}}^{b}$ maps $X_{0}(a, b) \subseteq X_{0} \times X_{0}^{F^{a-b}}$ into $\Delta \subseteq X_{0}^{F^{a}} \times X_{0}^{F^{a}}$; and $X_{0}(a, b)$ comes equipped with a finite, flat. radical, morphism

$$
\mathscr{D}_{0}(a, b): X_{0}(a, b) \rightarrow X_{0}
$$

To define

$$
\iota(a, b) \quad 0: X_{0}(a, b) \rightarrow Y_{0}
$$


we must specify a cyclic subgroup of order $p^{n}$ of $\mathscr{D}_{0}(a, b) * G_{0}^{\log }$. Now on the one hand, by composing the $a^{\text {th }}$ power of Frobenius with the $b^{\text {th }}$-power of the Verschiebung (as in $[\mathrm{KM}]$, Theorem 13.3.5), we get some subgroup object of order $p^{n}$ of $\mathscr{D}_{0}(a, b){ }^{*} G_{0}^{\log }$, and by the same argument as that given in $[\mathrm{KM}]$, Theorem 13.3.5, one sees that this subgroup must be cyclic (in the Drinfeldian sense). Thus, by the modular definition of $Y_{0}$, we get a morphism $\iota_{(a, b) .0}$ : $X_{0}(a, b) \rightarrow Y_{0}$.

In order to apply the theory of $[\mathrm{KM}]$, Chapter 13 , we must verify the conditions (1) through (8) listed at the beginning of that Chapter. (Caution: The letters $X$ and $Y$ in [KM], Chapter 13, are used in the reverse way to the way that they are used here.) Conditions (1), (2), (4), (5), and (6) are trivial. Condition (3) follows from the regularity of $Y$ and the fact that over a supersingular point, there is only one $A$-generator valued in $k$ for a cyclic group, namely the identity element. Note that at ordinary points, one can do the same analysis of $p^{\text {th }}$ power isogenies of $\log p$-divisible groups as is done in [KM] Chapter 13, §3. Thus, Condition (7) (that $\iota_{(a, b) .0}$ is a closed immersion) and Condition (8) (that the $\iota_{(a, b), 0}$ 's define an isomorphism of the disjoint union of $X_{0}(a, b)$ 's with $Y_{0}$ over the ordinary locus) follow at the level of topological spaces from this analysis, and at the level of complete local rings by considering the deformation parameters for the domain and range $\log p$-divisible groups of the isogeny. We thus get a result analogous to [KM], Theorem 13.4.7 :

Theorem 2.5. The $k$-scheme $Y_{0}$ is the disjoint union, with crossings at the supersingular points (in the terminology of $[K M]$, Chapter 13, §1), of the $n+1$ schemes $X_{0}(a, b)$ (where $\left.a+b=n\right)$. Let $f_{(a, b)} \in k[[x, y]]$ be the equation

$$
\left(x^{p^{a-1}}-y^{p^{b-1}}\right)^{p-1}
$$

if $a, b \geq 1$, and let it be $x^{p^{a}}-y^{p^{b}}$ if $a$ or $b$ is zero. Then the completed local ring at a $k$-rational supersingular point of $Y_{0}$ is isomorphic to

$$
k[[x, y]] /\left(\prod_{(a, b)} f_{(a, b)}\right)
$$

with the closed subscheme $X_{0}(a, b) \subseteq Y_{0}$ given by the equation $f_{(a, b)}$.

\section{§3. The Compactified Canonical Frobenius Lifting}

In this Section, we study the canonical Frobenius lifting on the ordinary locus of a canonical curve (defined in Theorem 1.6). In particular, we study its 
behavior at supersingular points, and "compactify it" in some sense, so as to obtain "pseudo-Hecke correspondences." It is by abstracting the main properties of this compactified Frobenius in the canonical case that we shall obtain a geometric criterion for a curve to be canonical in $\S 4$.

\section{The Canonical Frobenius $\mathbb{L}$ ifting and the Canonical $\mathbb{L}$ og $p$-divisible Group}

Let us denote by

$$
\bar{\Phi}_{X}^{\log }:\left(X^{\log }\right)^{\text {ord }} \rightarrow\left(\left(X^{\text {log }}\right)^{\text {ord }}\right)^{F}
$$

the canonical Frobenius lifting of Theorem 1.6. Let $G^{\log }$ be the canonical log $p$-divisible group on $X^{\mathrm{log}}$ of Definition 2.2. Then we rephrase Theorem 1.6 in terms of $G^{\text {log }}$ as follows:

Theorem 3.1. The canonical Frobenius lifting of Theorem 1.6

$$
\Phi_{X}^{\log }:\left(X^{\log }\right)^{\text {ord }} \rightarrow\left(\left(X^{\log }\right)^{\text {ord }}\right)^{F}
$$

induces an isogeny of degree $p$

$$
\left.\Phi_{X}^{*}\left(G^{\log }\right)^{F}\right|_{\left(X^{108}\right)_{\text {ord }}} \rightarrow G^{\left.\log \right|_{\left(X^{108}\right)^{\text {rrd }}}}
$$

between the canonical $\log p$-divisible groups that lifts the Frobenius morphism modulo $p$. Moreover, $\Phi_{X}^{\log }$ is the unique Frobenius lifting over $\left(X^{\log }\right)^{\text {ord }}$ that has this property.

Proof. The existence of the isogeny follows from the fact that we have defined a morphism between the respective Dieudonne crystals that respects the Hodge filtration. This induces the isogeny (see [BBM] and [Mess]). On the other hand, the uniqueness statement follows from the uniqueness statement in Theorem 1.6. together with the fact that if a Frobenius lifting induces such an isogeny. it automatically preserves the Hodge filtrations on the Dieudonné crystals.

Let $n \geq 0$. Let $Y=X_{0}(n)$. Let $Y^{\text {ord }} \subseteq Y$ be the $p$-adic open formal subscheme consisting of points lying over $X^{\text {ord }}$. Now the Frobenius lifting of Theorem 3.1 allows us to extend the decomposition in characteristic $p$ of $Y_{\mathbf{F}_{p}}$ into components corresponding to $(a, b)$-cyclic isogenies to a decomposition over 
$A=W(k)$, on the ordinary locus. To obtain this decomposition, we define closed $p$-adic subschemes

$$
X(a, b)^{\text {ord }} \subseteq X^{\text {ord }} \times_{A}\left(X^{\text {ord }}\right)^{F^{a-b}}
$$

via the same recipe as we did for $X_{0}(a, b)$, except using our canonical Frobenius lifting $\Phi_{X}^{\text {log }}:\left(X^{\text {log }}\right)^{\text {ord }} \rightarrow\left(\left(X^{\text {log }}\right)^{\text {ord }}\right)^{F}$ instead of $\Phi_{X_{0}}$. Then, just as before, we get a natural embedding $\iota_{(a, b)}: X(a, b)$ ord $\rightarrow Y^{\text {ord }}$ (analogous to $\iota_{(a, b), 0}$ ) which induces an isomorphism

$$
Y^{\text {ord }} \cong \prod_{a+b=n} X(a, b)^{\text {ord }}
$$

Finally, over $Y^{\text {ord }}$, we have a tautological isogeny

$$
G_{Y^{\text {ord }}}^{\log _{i}} \rightarrow H_{Y^{\text {IId }}}^{\log }
$$

(where $G_{Y \text { ord }}^{\log }$ is the pull-back of $G^{\log }$ to $Y^{\text {ord }}$ ) such that over $X(a, b)$ ord $H_{Y \text { us }}^{\text {log }}$ is naturally isomorphic to the pull-back of $\left(G^{\log }\right)^{F^{a-b}}$ via the projection of $X(a, b)^{\text {ord }} \rightarrow\left(X^{\text {ord }}\right)^{F^{a-b}}$ to the second factor.

\section{Local Analysis at Supersingular Points}

We now exploit the existence of the isogeny of Theorem 3.1 to understand the behavior of the canonical Frobenius lifting at the supersingular points. Let $x \in X(k)$ be a supersingular point. In studying $x$, we will often need to involve its various Frobenius conjugates $x^{F^{l}} \in X(k)$ (which may be infinite in number if the perfect field $k$ is not finite). We begin our analysis by considering the double iterate of the Frobenius morphism over some infinitesimal neighborhood $V \subseteq$ $X_{F_{p}}$ at $x$ :

$$
\Phi_{V}^{2}:\left.G^{\log _{\mid V}^{1}} \rightarrow G^{\log }\right|_{V^{F^{2}}}
$$

Thus, $V$ is the spectrum of a local artinian ring, with residue field $k$. Let us assume that $V$ is contained in the supersingular divisor (Chapter II, Proposition 2.6) of the canonical indigenous bundle. By definition, this means that over $V$, the Hodge filtration coincides with the FL-bundle filtration. It thus follows that over $V$, the kernels of the Verschiebung and Frobenius morphisms coincide. Since the kernel of the composite of the Verschiebung and the Frobenius is just the kernel of multiplication by $p$, it follows that the morphism $\Phi_{V}^{2}$ is isomorphic 
to the morphism "multiplication on p." In particular, it follows that

$$
\left.\left.G^{\log }\right|_{V^{F^{2}}} \cong G^{\log }\right|_{V}
$$

By iterating this isomorphism, we obtain that $\left.G^{\log }\right|_{V}$ is isomorphic to the pull-back to $V$ of a $p$-divisible group over $k$. Since the Kodaira-Spencer morphism of the Hodge filtration of $(\mathscr{E}, \nabla \mathscr{E})$ is an isomorphism, this implies that $V$ must be $\operatorname{Spec}(k)$. Thus, the assumption that $V$ lies inside the supersingular divisor implies that $V$ is reduced. Put another way, we see that we have proven (in this general context) the analogue of Igusa's theorem ( $[\mathrm{KM}], \mathrm{p} .355)$ :

Proposition 3.2. The supersingular divisor of the canonical indigenous bundle $\left(\mathscr{E}, \nabla_{\mathscr{E}}\right)$ is étale over $k$.

Next, let us observe that for any $x \in X(k)$, the completed local ring $R_{x}$ of $X$ at $x$ (which is formally smooth of dimension one over $A$ ) is naturally isomorphic to the universal deformation space of the $p$-divisible group $\left.G^{\log }\right|_{x}$. Indeed, it follows from the theory of [Mess] that deformations of $\left.G^{\log }\right|_{x}$ are given by deformations of the Hodge filtration; thus, our observation follows from the fact that the Kodaira-Spencer morphism of the Hodge filtration of $\left(\mathscr{E}, \nabla_{\mathscr{E}}\right)$ is an isomorphism. Now suppose that $x \in X(k)$ is supersingular. Then the isomorphism

$$
\left.\left.G^{\log }\right|_{x F^{2}} \cong G^{\log }\right|_{x}
$$

obtained above from the double iterate of Frobenius induces a natural isomorphism of complete local rings

$$
\Psi_{x}: R_{x F^{2}} \cong R_{x}
$$

which will play an important role in the sequel.

Now fix a number $n \geq 1$, and let $Y=X_{0}(n)$. If $x \in X(k)$, let us denote by $X_{x}$ the formal spectrum of $X$ at $x$, i.e., $\operatorname{Spf}\left(R_{x}\right)$. We will use similar notation for $Y$. Over $Y$, we have a tautological cyclic isogeny of order $p^{n}$.

$$
G_{Y}^{\log } \rightarrow H_{Y}^{\log }
$$

Fix a supersingular $x \in X(k)$. By the analysis of $[\mathrm{KM}]$, reviewed in $\S 2$. there exists a unique $y \in Y(k)$ lying over $x$. Now by thinking of the completed local rings of $X$ as universal classifying spaces, we obtain a morphism:

$$
\left(\mathscr{D}_{y}, \mathscr{R}_{y}\right): Y_{y} \rightarrow X_{x} \times_{A} X_{x^{F^{n}}}
$$


where $\mathscr{D}_{y}$ is the classifying morphism for the "domain $p$-divisible group" $G_{Y}^{\text {log }}$, and $\mathscr{R}_{y}$ is the classifying morphism for the "range $p$-divisible group" $H_{Y}^{\text {log }}$. Here we use the fact that restricted to $x$, the tautological isogeny is just the $n^{\text {th }}$ iterate of the Frobenius morphism, so $\left.H_{Y}^{\log }\right|_{y}=G_{x F^{n}}^{\log }$. Also, note that by the deformation theory of [Mess ᄀ, a deformation of the tautological isogeny is uniquely determined by the induced deformations of the domain and range $p$-divisible groups. It thus follows that the morphism $\left(\mathscr{D}_{y}, \mathscr{R}_{y}\right)$ is formally unramified, hence a closed immersion. Thus, henceforth, we shall think of $Y_{y}$ as a formal divisor inside $X_{x} \times X_{x F^{n}}$ by means of the closed immersion $\left(\mathscr{D}_{y}, \mathscr{R}_{y}\right)$.

One of the most important properties of this divisor $Y_{y}$ is its symmetry. More precisely, given $Y_{y}$, one can obtain a divisor in $X_{x F^{n}} \times X_{x}$ in two ways:

(1) by applying the isomorphism $X_{x} \times X_{x F^{n}} \cong X_{x F^{n}} \times X_{x}$ given by switching the two factors;

(2) by conjugating first by the $n^{\text {th }}$ power of Frobenius, so as to obtain a divisor in $X_{x F^{n}} \times X_{x F^{2 n}}$ and then applying the isomorphism $\operatorname{Spf}\left(\Psi_{x}\right)^{-n}$ to the second factor.

Then we claim that these two divisors in $X_{x F^{n}} \times X_{x}$ are the same. Indeed, to see this, it suffices to trace what happens to the tautological isogeny. Let us con sider the second procedure stated above. First, we conjugate the tautological isogeny by Frobenius:

$$
\left(G_{Y y}^{\log }\right)^{F^{n}} \rightarrow\left(H_{Y y}^{\log }\right)^{F^{n}}
$$

Since at $y$, this isogeny is just the $n^{\text {th }}$ iterate of the Frobenius morphism, by looking at Dieudonné modules, it follows that the kernel of this isogeny is contained in the kernel of multiplication by $p^{n}$. Thus, we get a morphism

$$
\left(H_{Y}^{\mathrm{log}}\right) F^{n} \rightarrow\left(G_{Y}^{\log }\right) F^{n}
$$

Since $\left(H_{y}^{\log }\right)^{F^{n}}=\left(G_{y}^{\log }\right) F^{2 n}$, we thus see that the divisor in $X_{x} F^{n} \times X_{x}$ obtained this way is just the divisor of isogenies (lifting the $n^{\text {th }}$ iterate of Frobenius) from the universal deformation of $G_{x}^{\log }$ (pulled back from the second factor) to the universal deformation of $\left(G_{x}^{\text {log }}\right)^{F^{n}}$ (pulled back from the first factor). On the other hand, if we look at the divisor obtained from the first procedure stated above, it admits exactly the same description. This proves the claim.

The next important property of this divisor $Y_{y} \subseteq X_{x} \times X_{x F^{n}}$ is that if we restrict it to the ordinary locus, it becomes equal to the union of the "local versions" of the schemes $X(a, b)$ ord (described at the end of the preceding 
subsection). More precisely, $X(a, b)^{\text {ord }}$ is defined as a closed subscheme of $X^{\text {ord }}$ $\times\left(X^{\text {ord }}\right)^{F^{a-b}}$. Thus, we obtain $\left.X(a, b)^{\text {ord }}\right|_{X_{x}} \subseteq\left(X^{\text {ord }} \times\left(X^{\text {ord }}\right)^{F^{a-b}}\right)_{X_{x}}$ by restricting to the formal scheme $X_{x}$. Since $a-b \equiv a+b=n(\bmod 2)$, by applying the appropriate power of $\Psi_{x}$, we thus obtain a subscheme, which we shall call

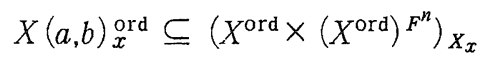

Then it follows from the functional definition of $Y$ (in terms of parametrizing isogenies) that

$$
\left.Y_{y}\right|_{X^{\text {ord }}}=\prod_{a+o=n} X(a, b)_{x}^{\text {ord }}
$$

Let us denote the supersingular divisor of $X$ by $X^{s s}$. We are now ready to summarize what we have done:

Definition 3.ə3. We shall call the pair

$$
\mathscr{H}_{x} \stackrel{\text { def }}{=}\left(Y_{y} ; \Psi_{x}\right)
$$

consisting of the divisor $Y_{y} \subseteq X_{x} \times X_{x F^{n}}$ and the isomorphism $\Psi_{x}$ the $n^{\text {th }}$ canonical local Hecke correspondence of $X^{\mathrm{log}}$ at $x$. We shall call the triple

$$
\left(Y \rightarrow X ; \Phi_{X}^{\log } ;\left\{\mathscr{H}_{x}\right\}_{x \in X}\right)
$$

the $n^{\text {th }}$ canonical pseudo-Hecke correspondence of $X^{\mathrm{log}}$.

Of course ideally, the local Hecke correspondences $\mathscr{H}_{x}$ would glue together to form a global Hecke correspondence, i.e., a morphism $Y \rightarrow X \times X^{F^{n}}$, just as in the classical case with modular curves. We shall investigate this issue in the next subsection, but (unfortunately) what we shall find is that the existence of such global Hecke correspondences is a rather rare phenomenon.

\section{Global Hecke Correspondences}

We maintain the notation of the previous subsection.

Definition 3.4. We shall say that the canonical curve $X^{\log }$ admits a global $n^{\text {th }}$ canonical Hecke correspondence if there exists a morphism 


$$
(\mathscr{D}, \mathscr{R}): Y \rightarrow X \times X^{F^{n}}
$$

that is equal to the local morphisms $\left(\mathscr{D}_{y}, \mathscr{R}_{y}\right)$ of the preceding subsection for every $y \in Y$ lying over a supersingular point of $X$. We say that $X^{\log }$ is of Hecke type if it admits a global $n^{\text {th }}$ canonical Hecke correspondence for every $n \geq 1$.

Proposition 3.5. Suppose that there exists an isomorphism $\Psi^{\log }: X^{\log } \rightarrow$ $\left(X^{-10 g}\right)^{F^{2}}$ that induces the morphism $\operatorname{Spf}\left(\Psi_{x}\right)$ when localized at every supersingular $x$ $\in X$. Then $X^{\log }$ is of Hecke type.

Proof. This follows immediately from gluing together $Y^{\text {old }}$ with the various $Y_{y}$ 's by means of $\Psi^{10 g}$.

Corollary 3.6. Suppose that $X^{\mathrm{log}}$, along with all of its supersingular points are defined over $\mathbb{F}_{p 2}$. Then $X^{\mathrm{log}}$ is of Hecke type.

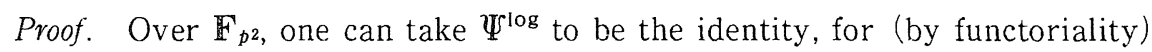
the $\Psi_{x}$ 's must all be the identity.

The classical example of a case where $X^{\text {log }}$ is of Hecke type is the case where $X^{\log }=\bar{M}_{1,0}^{\text {log }}$, the compactified moduli stack of elliptic curves (over $\mathbb{Z}_{p}$ ). This case is studied in detail in [Shi], Chapter 3. To see that the supersingular points are defined over $\mathbb{F}_{p^{2}}$, one repeats the argument preceding Proposition 3.2. to obtain an isomorphism $E^{F^{2}} \cong E$ for every supersingular elliptic curve $E$.

Suppose that $X^{\log }$ is canonical of Hecke type. Then we remark that just as in the classical case, one can define Herke opcrators on $H^{0}\left(X,\left(\omega_{X / S}^{\log _{S}}\right)^{\otimes N}\right)_{\mathbf{Q}_{p}}$ (as well as on the étale cohomology of $X_{Q_{p}}^{\mathrm{log}}$, etc.). Moreover. (by the same proofs as in the classical case) the effect on $q$-expansions (where $q$ is the Serre-Tate parameter at a marked point) is the same as in the classical case. (See, e.g., [Lang] for more details.)

Next, we justify the assertion (made in the preceding subsection) that, in some sense, being of Hecke type is a rather rare phenomenon. Indeed, if $X^{\text {log }}$ admits a global first canonical Hecke correspondence, consider its reduction modulo $p$ :

$$
(\mathscr{D}, \mathscr{R})_{\mathrm{F}_{p}}: Y_{\mathrm{F}_{p}} \rightarrow X_{\mathrm{F}_{p}} \times X_{\mathrm{F}_{p}}
$$

Let $Z=Y_{\mathbf{F}_{p}}$. Then $Z=Z^{\prime} \cup Z^{\prime \prime}$, with $Z^{\prime}$ mapping isomorphically to $X_{\mathbf{F}_{p}}$ via $\mathscr{D}_{\mathbf{F}_{p}}$, and $\left.\mathscr{D}_{\mathbf{F}_{p}}\right|_{Z^{\prime \prime}}$ isomorphic to the Frobenius morphism from $Z^{\prime \prime} \cong X_{\mathbf{F}_{p}}^{F^{-1}}$ to $X_{\mathbf{F}_{p}}$. On 
the other hand, $\mathscr{R}_{\mathrm{F}_{p}}$ must map $Z^{\prime \prime}$ isomorphically to $X_{\mathrm{F}_{p}}^{F}$. Thus, $Z^{\prime \prime}$ is isormorphic to both $X_{\mathrm{F}_{p}}^{F^{-1}}$ and $X_{\mathrm{F}_{p}}^{F}$. In particular, $X_{\mathrm{F}_{p}} \cong X_{\mathrm{F}_{p}}^{F^{2}}$, i.e., the moduli of $X_{\mathrm{F}_{p}}$ are defined over $\mathbb{F}_{p 2}$, which is a very rare phenomenon.

Remark. At the present time, the author does not know of any canonical $X^{\mathrm{log}}$ of Hecke type, except for those that arise directly from the modular case.

\section{§4. $p$-Adic Green's Frnetions}

In this Section, we give a geometric criterion for a curve to be canonical: namely, the existence of a Frobenius lifting of the right height, over an open $p$-adic formal subscheme of the curve, with "nice behavior" at the points where it is not defined. We will make these terms precise below, but the point of in terest is that this criterion does not depend on knowing the action of the canonical Frobenius $\Phi_{\mathcal{N}}$ on $\overline{\mathcal{N}}_{\boldsymbol{g}, \boldsymbol{r}}^{\text {ord }}$ - that is, it is intrinsic to the curve - and, moreover, it is not phrased in terms of indigenous bundles. Now in the case of elliptic curves (regarded parabolically), the canonical lifting defined in terms of indigenous bundles is the same as the canonical lifting defined in Serre-Tate theory (one of the definitions of which is the existence of a global Frobenius lifting). Thus, it is interesting to note that the existence of an "admissible Frobenius lifting" amounts to just the existence of a Frobenius lifting (in the case of elliptic curves). In other words, one may regard the geometric criterion given here as the proper hyperbolic generalization of the statement that an elliptic curve (whose reduction modulo $p$ is ordinary) is Serre-Tate canonical if and only if it admits a Frobenius lifting.

\section{Compactified Frobenius Liftings}

In this subsection, motivated by the construction of the pseudo-Hecke correspondences in the previous Section, we define the general notion of a "compactified Frobenius lifting." Let $X^{\log } \rightarrow S^{\log }$ be a smooth $r$-pointed curve of genus $g$. Let

$$
\phi^{\log }: Y^{\log } \rightarrow X^{\log }
$$

be a finite, flat morphism such that $Y$ is regular (necessarily of dimension two), and the log structure on $Y^{\log }$ is defined by a divisor on $Y$ which is étale over $A$ and equal to the set-theoretic inverse image of the divisor of marked points of $X$. Let $U \subseteq X$ be an open formal subscheme that contains all the marked points. We endow $U$ with the log structure pulled back from $X^{\text {log }}$, and call the resulting 
$\log$ formal scheme $U^{\log }$. We shall use the notation $X_{x}, Y_{y}$, etc. (as in the previous subsection) to denote the formal schemes which are the formal neighborhoods of the closed points $x, y$, etc. Suppose that

$$
\Phi^{\log }: U^{\log } \rightarrow\left(U^{\log }\right)^{F}
$$

is a Frobenius lifting.

Definition 4.1. We shall say that $\left(\phi^{\log }: Y^{\log } \rightarrow X^{\log }: \iota\right)$ is a naive compact ification of the Frobenius lifting $\Phi^{\log }$ if, when we take $V=\phi^{-1}(U)$, the following conditions are satisfied:

(1) $V^{\log }$ splits as a disjoint union $\left(V^{\log }\right)^{\prime} \amalg\left(V^{\log }\right)^{\prime \prime}$;

(2) $\left.\phi^{\log }\right|_{V^{\prime}}:\left(V^{\log }\right)^{\prime} \rightarrow U^{\log }$ is an isomorphism;

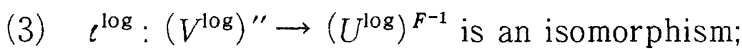

(4) $\left.\phi^{\log }\right|_{V^{\prime \prime}}-\left(\iota^{\log }\right)^{-1}:\left(U^{\log }\right)^{F^{-1}} \rightarrow U^{\log }$ is the morphism $\left(\Phi^{\log }\right)^{F^{-1}}$.

We shall frequently identify $V^{\prime}$ and $U$, and $V^{\prime \prime}$ and $U^{F-1}$.

Suppose that $\left(\phi^{\log } ; \ell^{\log }\right)$ is a naive compactification for $\Phi^{\log }$. Note that $\phi$ is necessarily of degree $p+1$. Also, note that $V^{\prime}$ admits a canonical embedding

$$
\iota_{(1,0)}: V^{\prime} \hookrightarrow U \times U^{F}
$$

where we take $\iota_{(1,0)}=(\mathrm{id}, \Phi)$, while $V^{\prime \prime}$ admits an embedding

$$
\iota_{(0,1)}: V^{\prime \prime} \hookrightarrow U \times U^{F^{-1}}
$$

where we take $\iota_{(0,1)}=\left((\Phi)^{F^{-1}}\right.$, id $)$.

Let $x \in X(k)$. Let

$$
\Psi_{x}: R_{F^{F^{2}}} \cong R_{x}
$$

be an isomorphism. Suppose that $y \in Y(k)$ maps to $x$. Let

$$
\left(\mathscr{D}_{y}, \mathscr{R}_{y}\right): Y_{y} \hookrightarrow X_{x} \times X_{x} F
$$

be a closed embedding, where $\mathscr{D}_{y}$ is obtained by restricting $\phi$.

Definition 4.2. We shall say that $\mathscr{H}_{x} \stackrel{\text { def }}{=}\left(\Psi_{x} ;\left(\mathscr{D}_{y}, \mathscr{R}_{y}\right)\right)$ is a local compacti- 
fication for the Frobenius lifting $\Phi^{\log }$ at $x$ (relative to $\left(\phi^{\log } ; c^{\log }\right)$ ) if

(1) $y$ is the unique closed point of $Y$ lying over $x$;

(2) the divisor $Y_{y} \hookrightarrow X_{x} \times X_{x^{F}}$ is symmetric in the sense that the two divisors that it induces in $X_{x F} \times X_{x}$ (by switching and by Frobeniusconjugating, then applying $\left.\Psi_{x}\right)$ are the same;

(3) the restriction of $\left(\mathscr{D}_{y}, \mathscr{R}_{y}\right)$ to $V$ is the union of $\iota_{(1,0)}$, and $\left(\iota_{(0,1)}\right)^{F^{2}}$ composed with $\operatorname{Spf}\left(\Psi_{x}\right)^{-1} \times($ id).

Note that by the first condition, $x$ cannot lie in $U$. Now let us consider $\left(Y_{y}\right)_{\mathrm{z} / p^{2} \mathrm{Z}}$. Since $Y_{y} \subseteq X_{x} \times X_{x F}$, it follows that $\left(Y_{y}\right)_{\mathrm{Z} / p^{2} \mathrm{Z}}$ is Spf of a local ring of the form:

$$
R \stackrel{\text { def }}{=}\left(A / p^{2} A\right)[[\xi, \eta]] / \phi(\xi, \eta)
$$

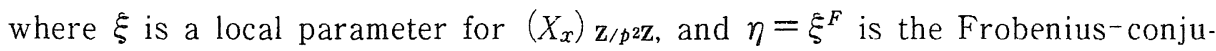

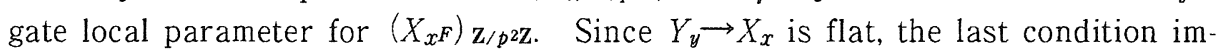
plies that $\left(Y_{y}\right)_{\mathbf{F}_{p}}$ has exactly two irreducible components, both of which are reduced. Thus, if we denote by a "bar" the reduction of functions modulo $p$, we see that $\bar{\phi}$ is a product of two distinct prime elements of $k[[\bar{\xi}, \bar{\eta}]]$. In fact, we can say more. Outside the special point of $\left(Y_{y}\right)_{\mathbf{F}_{p}}$, these two primes define the closed subschemes $\bar{\xi}^{p}-\bar{\eta}$ and $\bar{\eta}^{p}-\Psi_{x}\left(\bar{\xi}^{F^{2}}\right)$. Let

$$
f=\xi^{p}-\eta ; g=\eta^{p}-\Psi_{x}\left(\xi^{F^{2}}\right)
$$

Thus, we may assume that $\bar{\phi}=\bar{f} \cdot \bar{g}$. In other words, we can write

$$
\phi=f \cdot g+\pi
$$

where $\pi \in p \cdot k[[\bar{\xi}, \bar{\eta}]]$. In fact, $\pi$ is actually $p$ times a unit in $k[[\bar{\xi}, \bar{\eta}]]$, since $Y_{y}$ is regular.

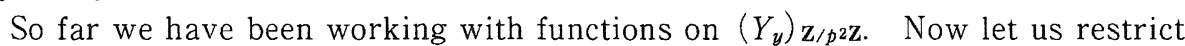
to functions on the open formal subscheme $D(g) \subseteq\left(Y_{y}\right)_{\mathbf{z} / p^{2} \mathbf{Z}}$ (i.e., where $g$ is invertible). Thus, we are in effect restricting to the graph of $\Phi$. Let us denote the restriction morphism on functions by $\zeta: R \rightarrow R[1 / g]$. Then we obtain, in $R[1 / g]$,

$$
\zeta(\eta)=\zeta(\xi)^{p}+\zeta(\pi) \cdot \zeta(g)^{-1}
$$

By interpreting this open formal subscheme $D(g)$ as the graph of $\Phi$, this tells 
us that $\Phi^{-1}\left(\xi^{F}\right)$ is a function which is not regular at $x$, but has a pole of order one (since $\bar{g}$ has a zero of order one). In particular, it tells us that the Frobenius lifting $\Phi$ does not admit a regular extension to any neighborhood of $x$. We summarize this as follows:

Proposition 4.3. If $\mathscr{H}_{x}$ is a local compactification of $\Phi^{\log }$ at $x$, then $\left(Y_{y}\right)_{\mathbf{F}_{\mathrm{p}}}$ is a node, and $x \notin U$. Also, the Frobenius lifting $\Phi$ does not admit an extension to any neighborhood of $x$.

Definition 4.4. We shall call

$$
\mathscr{C}=\left(\phi^{\log } ; \iota^{\log } ;\left\{\mathscr{H}_{x}\right\}_{x \notin U}\right)
$$

a compactification of the Frobenius lifting $\Phi^{10 \mathrm{~g}}$ if $(\phi ; \iota)$ is a naive compactification of $\Phi^{10 g}$, and for each $x \notin U$, we are given a local compactification $\mathscr{H}_{x}$ of $\Phi^{\log }$ relative to $(\phi ; c)$ (where $k$ in the definition above is replaced by the field of rationality of $x$ ).

Thus, in particular. by what we did in the last two subsections,

Proposition 4.5. Suppose that $X^{\mathrm{Iog}}$ is a canonical curve. Then its first canonical pseudo-Hecke correspondence is a compactification of the canonical Frobenius lifting on $\left(X^{\mathrm{log}}\right)^{\text {ord }}$.

Suppose that $\left(\phi^{\log } ; \iota^{\log } ;\left\{\mathscr{H}_{x}\right\}_{x \notin U}\right)$ is a compactification of $\Phi^{\log }$. Let us consider $Z \stackrel{\text { def }}{=} Y_{\mathbf{F}_{p}}$. It follows from the above definition that $Z$ is reduced and has exactly two irreducible components $Z^{\prime}$ and $Z^{\prime \prime}$ with $V_{\mathbf{F}_{p}}^{\prime} \subseteq Z^{\prime} ; V^{\prime \prime}{ }_{\mathbf{F}_{p}} \subseteq Z^{\prime \prime}$. Since $Z^{\prime}$ is geometrically connected, smooth, proper, and birationally equivalent to $X \mathbf{F}_{p}$ over $k$, it follows that $Z^{\prime} \cong X \mathbf{F}_{p}$. Similarly. $Z^{\prime \prime} \cong X_{\mathbf{F}_{p}}^{F^{-1}}$. Moreover, except at the points of intersection of $Z^{\prime}$ and $Z^{\prime \prime}$ (which are nodes), $Z$ is smooth over $k$.

Proposition 4.6. (Assuming that $X^{\mathrm{log}}$ is hyperbolic) $Y$ must be connected.

Proof. It suffices to prove that $Z$ is connected. Suppose that $Z$ is not connected. Then $\phi_{Z^{\prime}}: Z^{\prime} \rightarrow X_{\mathbf{F}_{p}}$ is finite and birational, hence an isomorphism. It thus follows that $Z^{\prime}$ lifts to a connected component $Y^{\prime \prime}$ of $Y$ such that $\phi_{Y^{\prime}}: Y^{\prime} \rightarrow X$ is an isomorphism. On the other hand, $Z^{\prime \prime}$ is proper and smooth over $k$, and birational to $X_{\mathbf{F}_{p}}^{F^{-1}}$, hence $Z^{\prime \prime} \cong X_{\mathbf{F}_{p}}^{F_{p}^{-1}}$, and $\left.\phi\right|_{\mathbf{Z}^{\prime \prime}}: Z^{\prime \prime} \rightarrow X_{\mathbf{F}_{p}}$ is the Frobenius morphism. Moreover, $Z^{\prime \prime}$ lifts to a connected component $Y^{\prime \prime}$ of $Y$. Thus, $\phi Y_{Y^{\prime \prime}}: Y^{\prime \prime} \rightarrow$ 
$X$ is a Frobenius lifting. But if $X^{\log }$ is hyperbolic, such Frobenius liftings cannot exist, for the nonzero morphism of line bundles $\left(\left.\phi\right|_{Y^{\prime \prime}}\right)^{*} \omega_{X / S}^{\log } \rightarrow \omega_{Y^{\prime \prime} / S}^{\log }$ violates degree restrictions.

\section{The Height of a Frobenius Lifiting}

Finally, we note that often it is useful to have a precise measure of how far a Frobenius lifting fails to extend over all of $X$. For this, we introduce the notion of the height of a Frobenius lifting, as follows.

Let $\mathscr{F}_{F} \rightarrow X_{\mathbf{F}_{p}}$ be the $\mathscr{T}_{\mathbf{F}_{p}}$-torsor of Frobenius liftings on open sub-log

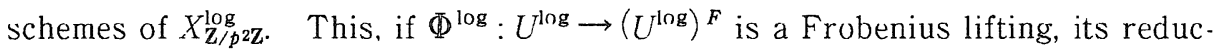
tion modulo $p^{2}$ defines a section $\sigma_{\Phi}: U_{F_{p}} \rightarrow \mathscr{F}^{\prime}$ of this torsor. Let $P$ be the projective bundle that canonically compactifies $\mathscr{F}$. Thus, $P \rightarrow X_{\mathrm{F}_{p}}$ is a $\mathbb{P}^{1}$-bundle. Recall the notion of the canonical height of a section of $P \rightarrow X_{\mathrm{F}_{p}}$, introduced at the beginning of Chapter I, $\S 2$. Since $X_{\mathrm{F}_{p}}$ is proper over $k$, it follows that $\sigma_{\Phi}$ extends uniquely to a section $\bar{\sigma}_{\Phi}: X_{F_{p}} \rightarrow P$. We now make the following.

Definition 4.7. We define the height ht $(\Phi)$ of the Frobenius lifting $\Phi^{\log }$ to be the canonical height of the section $\bar{\sigma}_{\Phi}$ of $P \rightarrow X_{\mathbf{F}_{p}}$.

More concretely, the height of $\Phi^{\log }$ can be defined as follows. If $x \in X_{\mathbf{F}_{p}}^{\log }$. then let $t$ be a local parameter of $X^{\mathrm{log}}$ at $x$. Let $\Xi_{x}$ be a local Frobenius lifting defined in a neighborhood of $x$. Then $\frac{1}{p}\left(\Xi_{x}^{-1}(t)-\Phi^{-1}(t)\right)$ is a rational function $\delta_{x}$ on $X_{\mathrm{F}_{p}}^{\log }$. Let us say that the local height ht ${ }_{x}(\Phi)$ of $\Phi^{\log }$ at $x$ is:

(1) equal to 0 if this function $\delta_{x}$ is regular at $x$;

(2) equal to the order of the pole of $\delta_{x}$ at $x$ otherwise.

Then we have the formula:

Proposition 4.8. We have

$$
\mathrm{ht}(\Phi)+\frac{p}{2}(2 g-2+r)=\sum_{x \in X_{F_{p}}^{\text {IOg }}}[k(x): k] \mathrm{ht}_{x}(\Phi)
$$

Proof. This follows immediately from considering the intersection number of $\bar{\sigma}_{\Phi}$ with the "section at infinity" given by the complement of $\mathscr{F}$ in $P$.

Corollary 4.9. If $\Phi^{\log }$ admits a compactification, then the local heights at points outside $U$ are all one. Thus, 


$$
\text { ht }(\Phi)=-\frac{p}{2}(2 g-2+r)+\operatorname{deg}_{k}(X-U)_{\mathrm{F}_{p}}
$$

where we regard $(X-U)_{\mathrm{F}_{p}}$ as having the reduced, induced scheme structure.

Proof. The statement about local heights follows from the explicit computation preceding Proposition 4.3.

The following is the main result of this subsection:

Proposition 4.10. If $\Phi^{\log }$ has height $\leq 1-g-\frac{1}{2} r$, then $P$ (with its connection $\nabla_{P}$ induced by that of $\left.\mathscr{F}\right)$ is a nilpotent, admissible indigenous bundle. In particular, if $\left(P, \nabla_{P}\right)$ is also ordinary, then $X^{\log }$ is isomorphic to a canonical curve modulo $p^{2}$, and $\Phi^{\log }$ is equal to the canonical Frobenius lifling (of Theorem 3.1) modulo $p^{2}$.

Proof. Indeed, suppose that ht $(\Phi) \leq 1-g-\frac{1}{2} r$. Consider the KodairaSpencer morphism of the section $\bar{\sigma}_{\Phi}$ of $P \rightarrow X$. By the general properties of FL-bundles (Chapter II, §1), we know that the Kodaira-Spencer morphism cannot vanish (for the section at infinity of $P \rightarrow X$ is the unique horizontal section). But by degree considerations (i.e., the assumption on ht $(\Phi)$ ), once the Kodaira-Spencer morphism is nonzero, it must be an isomorphism. It thus follows that $P$ (with its connection induced by that of $\mathscr{F}$ ) is a nilpotent, admissible indigenous bundle. The last statement follows from the construction of the canonical lifting and the canonical Frobenius.

Thus, we see that the compactified Frobenius liftings that we are really interested in are the ones that "look nice modulo $p:$ "

Definition 4.11. A compactified Frobenius lifting

$$
\mathscr{C}=\left(\phi^{\log } ; \iota^{\log } ;\left\{\mathscr{H}_{x}\right\}_{x \notin U}\right)
$$

is called admissible if

(1) $\operatorname{ht}(\Phi)=g-1+\frac{1}{2} r$;

(2) the associated $\left(P, \nabla_{P}\right)$ (as in Proposition 4.10) is ordinary;

(3) the reductions modulo $p$ of the isomorphisms $\Psi_{x}$ (that make up $\mathscr{H}_{x}$ ) 
are equal to the canonical " $\Psi_{x}$ " of Definition 3.3.

Note that for an admissible compactified Frobenius $\mathscr{C}$. all the objects involved (that is, $\Phi^{\log } ; \phi^{\log }: Y^{\log } \rightarrow X^{\log } ; \iota^{\log } ; \Psi_{x} ;\left(\mathscr{D}_{y}, \mathscr{R}_{y}\right)$ ) are completely determined modulo $p$ (up to isomorphism) once one fixes the supersingular divisor $(X-U)_{F_{p}}$. Or, in other words,

Proposition 4.12. An admissible compactified Frobenius $\mathscr{C}$ on $X^{\text {log }}$ deter. mines a p-adic quasiconformal equivalence class $\alpha$ to which $X^{\text {log }}$ belongs. If two admissible compactified Frobenius $\mathscr{C}$ and $\mathscr{C}^{\prime}$ on $X^{\mathrm{log}}$ determine the same $\alpha$, then, modulo $p$, all the objects that make up $\mathscr{C}$ are isomorphic to those that make up $\mathscr{C}^{\prime}$.

\section{Admissible Frobenius Liftings}

Since an admissible compactified Frobenius is determined modulo $p$ by the $p$-adic quasiconformal equivalence class $\alpha$, the next step is to understand what the possible deformations looks like. Let $\mathscr{C}$ be an admissible compactified Frobenius, and let us consider $\mathscr{C}_{\mathbf{z} / p^{2} \mathbf{Z}}$, i.e., the reductions modulo $p^{2}$ of all the objects involved. Suppose we start with the data $\left(\Phi^{10 g}\right)_{\mathrm{Z} / \mathbf{p}^{2} \mathbf{Z}} ;\left(\Psi_{x}\right)_{\mathrm{Z} / \mathbf{p}^{2} \mathbf{Z}}$ (for all supersingular $x$ ). Now it is easy to see (by looking at points valued in an arbitrary scheme) that the symmetry condition on the divisor $Y_{y}$ amounts to the statement that $\left(\Psi_{x}\right)_{\mathrm{Z} / \mathrm{p}^{2 \mathrm{Z}} \mathrm{Z}}$ restricted to the ordinary locus commutes with $\Phi_{\mathrm{Z} / p^{2 Z} \mathrm{Z}}$.

Let $\beta$ be an automorphism of $\left(X_{x}\right)_{\mathrm{z} / \mathrm{p} 2 \mathrm{Z}}$ which is equal to the identity modulo $p$. Then we claim that if $\Phi_{\mathbf{Z} / p_{2} \mathbf{Z}}$ commutes with $\beta$, then $\beta$ is the identity. Indeed. since derivations on $X_{\mathbf{F}_{p}}$ act trivially on functions that are $p^{\text {th }}$ powers, we get that

$$
\Phi_{\mathrm{Z} / p^{2 Z} \mathbf{Z}} \beta=\Phi_{\mathrm{Z} / p^{2} \mathbf{Z}}
$$

Thus,

$$
\beta^{F}: \Phi_{\mathrm{Z} / p 2 \mathrm{Z}}=\Phi_{\mathrm{Z} / p^{2 \mathrm{Z}}}
$$

which implies that $\beta$ is the identity, since $\Phi_{{\mathrm{Z} / p^{2} \mathrm{Z}}}$ is faithfully flat. Thus, in summary, the $\left(\Psi_{x}\right)_{\mathrm{Z} / p^{2} \mathrm{Z}}$ are determined uniquely by the condition that they commute with $\Phi_{\mathrm{Z} / p^{2} \mathrm{Z}}$.

Next, we consider $Y^{\text {log }}$. We can break $Y^{\text {log }}$ up into three parts: $\left(V^{\text {log }}\right)^{\prime}$; $\left(V^{\log }\right)^{\prime \prime}$; and the $Y_{y}^{\prime}$ 's. Since $\left(V^{\text {tog }}\right)^{\prime}$ and $\left(V^{\log }\right)^{\prime \prime}$ are determined up to natural isomorphism by $X^{\mathrm{log}}$, it remains to determine the $Y_{y}$ 's, and the gluing morphisms. But $Y_{y}$ and its gluing morphisms to $\left(V^{\log }\right)^{\prime}$ and $\left(V^{\log }\right)^{\prime \prime}$ are completely 
specified once one knows the divisor $Y_{y}$ in $X_{x} \times X_{X^{F}}$. Moreover, it follows from the condition (3) of Definition 4.2, that this divisor is determined by $\Phi$ and $\Psi_{x}$. But we just saw that $\left(\Psi_{x}\right)_{\mathrm{Z} / p^{2 Z} \mathrm{Z}}$ is determined by $\Phi_{\mathrm{Z} / p^{2} \mathrm{Z} \text {. Thus, we conclude that }}$ $\left(\mathscr{H}_{x}\right)_{\mathbf{Z}_{/ p^{2}} \mathbf{Z}}$ and $\phi_{\mathbf{Z}_{/ p^{2} \mathbf{Z}}}^{\log }$ are completely determined by $\Phi_{\mathbf{Z}_{/ p^{2} \mathbf{Z}} \text {. Sorting through all }}$ the definitions, we thus see that we have proven that $\mathscr{C}_{\mathbf{z} / p^{2} \mathbf{Z}}$ is entirely determined by the $p$-adic quasiconformal equivalence class $\alpha$ and the deformation $\Phi_{\mathrm{Z} / p^{2} \mathbf{Z}}$. Moreover, there is nothing special about working modulo $p^{2}$ : the same arguments can be made modulo an arbitrary power of $p$. Thus, we see that we have proven the following result:

Lemma 4.13. Let $\mathscr{C}$ be an admissible compactified Frobenius lifting on $X^{\mathrm{log}}$. Then $\mathscr{C}_{\mathbf{Z} / p^{n \mathrm{Z}}}$ is completely determined by $\Phi_{\mathbf{Z} / p^{n} \mathrm{Z}}^{\mathrm{log}}$.

This Lemma suggests the following definition:

Definition 4.14. Let $\alpha$ be a $p$-adic quasiconformal equivalence class to which $X^{\text {log }}$ belongs. Let $U \subseteq X$ be the ordinary locus for $\alpha$. Let $\Phi^{\log }: U^{\log } \rightarrow$ $\left(U^{\log }\right)^{F}$ be a Frobenius lifting over $U^{\log }$. Then we shall say that $\Phi^{\log }$ is an admissible Frobenius lifting for $\left(X^{\log }, \alpha\right.$ ) if it arises from a (necessarily unique) admissible compactified Frobenius $\mathscr{C}$.

Thus, we can regard admissible Frobenius liftings as being Frobenius liftings (over the ordinary locus) that happen to have special behavior near the supersingular points.

Next let us consider two admissible Frobenius liftings $\Phi^{\log }$ and $\left(\Phi^{\log }\right)^{\prime}$ on the same curve $X^{\mathrm{log}}$. Let us suppose that they are equal modulo $p^{n}$ (where $n \geq$ 2 ). In this discussion, we shall always be working modulo $p^{n+1}$, so (by abuse of notation) we shall use $\Phi^{\log }$ and $\left(\Phi^{\log }\right)^{\prime}$ to denote the respective reductions modulo $p^{n+1}$. Now just as in the discussion preceding Proposition 4.3. for $\Phi^{\text {log }}$. $\left(Y_{y}\right)_{\mathrm{Z} / p^{n+1} \mathrm{Z}}$ is Spf of a local ring of the form:

$$
R \stackrel{\text { def }}{=}\left(A / p^{n+1} A\right)[[\xi, \eta]] / \phi(\xi, \eta)
$$

where $\xi$ is a local parameter for $\left(X_{x}\right)_{\mathrm{z}^{\prime} p^{n+1} \mathrm{Z}}$, and $\eta=\xi^{F}$ is the Frobenius-conju-

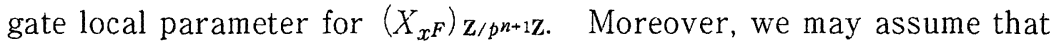

$$
\psi=f \cdot g+\pi
$$

where 


$$
f=\xi^{p}-\eta: g=\eta^{p}-\Psi_{x}\left(\xi^{F^{2}}\right)
$$

and $\pi \in p \cdot\left(A / p^{n} A\right)[[\bar{\xi}, \bar{\eta}]]$. In fact, $\pi$ is actually $p$ times a unit, since $Y_{y}$ is regular. Similarly, for $\left(\Phi^{\text {log }}\right)^{\prime}$, we have

$$
R^{\prime} \stackrel{\text { def }}{=}\left(A / p^{n+1} A\right)[[\xi, \eta]] / \psi^{\prime}(\xi, \eta)
$$

with $\psi^{\prime}=f \cdot g^{\prime}+\pi^{\prime}$, and $g^{\prime}=\eta^{p}-\Psi_{x}^{\prime}\left(\xi^{F^{2}}\right)$. Thus,

$$
g \equiv g^{\prime}\left(\bmod p^{n}\right) ; \pi \equiv \pi^{\prime}\left(\bmod p^{n}\right)
$$

So far we have been working with functions on $\left(Y_{y}\right) \mathrm{z}_{/} p^{n+1} \mathrm{z}$. Now let us restrict to the open subscheme corresponding to the graph of $\Phi$ (or $\Phi^{\prime}$ ). Let us denote the restriction morphism on functions by $\zeta$. Then we obtain, on this open subscheme, in the unprimed case:

$$
\zeta(\eta)=\zeta(\xi)^{p}+\zeta(\pi) \cdot \zeta(g)^{-1}
$$

and, in the primed case:

$$
\begin{aligned}
\zeta(\eta) & =\zeta(\xi)^{p}+\zeta\left(\pi^{\prime}\right) \cdot \zeta\left(g^{\prime}\right)^{-1} \\
& =\zeta(\xi)^{p}+\zeta\left(\pi^{\prime}\right) \cdot \zeta(g)^{-1}
\end{aligned}
$$

since the difference between $g$ and $g^{\prime}$ becomes zero when multiplied by $p$. Ulti. mately, we are interested in computing the difference between the two Frobenius liftings $\Phi$ and $\Phi^{\prime}$. That is, we wish to understand the difference between where $\eta$ is taken by the two liftings. But by the above formulas, the difference is of the form:

$$
\left\{\zeta(\pi)-\zeta\left(\pi^{\prime}\right)\right\}(\zeta(g))^{-1}
$$

Moreover, because the difference in brackets is divisible by $p^{n}$, only the residue of $g$ modulo $p$ is involved in the above expression. Since this residue has a zero of order exactly one at $x$, it follows that the difference between the two Frobenius liftings - which forms a section of $\mathscr{T}_{F_{p}}$ over $X^{\text {ord }}$ - has poles of order at most one at the supersingular points. Since the morphism

$$
H \mathscr{T}_{\mathbf{F}_{p}}: \mathscr{T}_{\mathbf{F}_{p}} \rightarrow\left(\tau_{X^{108} / S^{108}}\right)_{\mathbf{F}_{p}}
$$


(given by composing the $p$-curvature of $\left(P, \nabla_{P}\right)$ with the projection given by the Hodge filtration) has zeroes at the supersingular points, it thus follows that the difference between $\Phi^{\log }$ and $\left(\Phi^{\log }\right)^{\prime}$ defines a global section of $\left(\tau_{X^{108} / S^{108}}\right)_{F_{p}}$. Since we are dealing with hyperbolic curves. though, $\left(\tau_{\boldsymbol{X}^{108} / S^{108}}\right) F_{p}$ has negative degree, hence has no global sections. Thus, $\Phi^{10 g}=\left(\Phi^{\log }\right)^{\prime}$. In summary, we have proven the following strengthened form of Lemma 4.13:

Lemma 4.15. If there exists an admissible Frobenius lifting of $\left(X^{\log }, \alpha\right)$, then it is unique, and admits no nontrivial deformations modulo any power of $p$.

\section{Geometric Criterion for Canonicality}

Let us fix $X^{\log } \rightarrow S^{\log }$, a smooth $r$-pointed curve of genus $g$, and a $p$-adic quasiconformal equivalence class $\alpha$ to which $X^{\text {log }}$ belongs. Let $\Phi^{\log }$ be an admissible Frobenius lifting for $\left(X^{\log }, \alpha\right)$.

Now suppose that $\widetilde{X}^{\log } \rightarrow S^{\log }$ is also a smooth $r$-pointed curve of genus $g$ such that $\left(\widetilde{X}^{\log }\right)_{\mathbf{F}_{p}}=X_{\mathbf{F}_{p}}^{\log }$. The following "Rigidity Lemma" is fundamental to this subsection:

Lemma 4.16. Suppose that $\left(\widetilde{X}^{\mathrm{log}}, \alpha\right)$ admits an admissible Frobenius lifting $\left(\Phi^{\log }\right)^{\prime} . \quad$ Then $\widetilde{X}^{\mathrm{log}}=X^{\log }$.

Proof. We propose to prove inductively (on $n$ ) the following statement:

$$
(*)\left(\bar{X}^{\log },\left(\Phi^{\log }\right)^{\prime}\right) \text { coincides with }\left(X^{\log }, \Phi^{\log }\right) \text { modulo } p^{n+1}
$$

We know that this statement holds for $n=0$. Assume that it holds for $n-1$. Consider the difference between the deformations $X_{\mathbf{Z} / p^{n+1} \mathbf{Z}}^{\log }$ and $\widetilde{X}_{\mathbf{Z} / p^{n+1} \mathbf{Z}}^{\text {log }}$ of $X_{\mathbf{Z} / p n \mathbf{Z}}^{\log }$. It defines a class

$$
\mu \in H^{1}\left(X_{\mathbf{F}_{p}},\left(\tau_{X^{108} / S^{108}}\right)_{\mathbf{F}_{p}}\right)
$$

Let $\mathscr{C}$ (respectively, $\mathscr{C}^{\prime}$ ) be the compactified Frobenius corresponding to $\Phi^{\text {log }}$ (respectively, $\left.\left(\Phi^{\log }\right)^{\prime}\right)$. Since $Y_{Z^{\prime} / p n Z}^{\log }=\left(Y^{\log }\right)^{\prime}{ }_{\mathbf{Z} / p n Z}$, we can consider the differ-

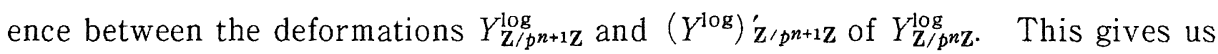
a class

$$
\nu \in \operatorname{Ext}_{Y_{F_{p}}}^{1}\left(\Omega_{Y_{F_{p}}^{108} / k}^{1}, \mathscr{O}_{Y_{F_{p}}}\right)
$$


Since $\phi_{\mathbf{Z} / p^{n \mathbf{Z}}}=\phi^{\prime} \mathbf{Z}_{/ p n \mathbf{Z}}$, the pull-back map defined by either of these morphisms gives us a map $\phi^{-1}: \phi^{*}\left(\omega_{X / S}^{\log }\right)_{F_{p}} \rightarrow \Omega_{Y_{F_{p} / k}^{\text {log }}}$, which is generically zero over the component of $Y_{\mathrm{F}_{p}}$ that we called $Z^{\prime \prime}$ in the proof of Proposition 4.6. Now $\phi^{-1}$ induces a pull-back morphism on global Ext's

$$
\phi: \operatorname{Ext}_{Y_{F_{p}}}^{1}\left(\Omega_{Y_{F_{p}}^{108} / k}^{1}, \mathscr{O}_{Y_{F_{p}}}\right) \rightarrow H^{1}\left(Y_{F_{p}} . \phi^{*}\left(\tau_{X^{108} / S^{108}}\right)_{F_{p}}\right)
$$

The condition that the morphism $\phi_{\mathrm{Z} / p^{n+1} \mathrm{Z}}$ deform compatibly with the deformation of $\left(X^{\log }, Y^{\log }\right)_{\mathbf{Z} / p^{n+1} \mathbf{Z}}$ to $\left(\widetilde{X}^{\log },\left(Y^{\prime}\right)^{\log }\right)_{\mathbf{Z} / p^{n+1} \mathbf{Z}}$ to a morphism $\phi_{\mathbf{Z} / p^{n+1} \mathbf{Z}}^{\prime}$ is exactly that

$$
\phi(\nu)=\left.\mu\right|_{Y_{F_{p}}}
$$

Note that this condition, as well as the cohomology modules in which $\mu$ and $\nu$ live. are independent of $n$. Thus, by adding $\mu$ and $\nu$ to $\phi_{\mathbf{Z} / p^{2} \mathbf{Z}}^{\log }: Y_{\mathbf{Z}^{\prime} / p^{2} \mathbf{Z}}^{\log } \rightarrow X_{\mathbf{Z} / p^{2} \mathbf{Z}}^{\log }$, we obtain a new morphism $\hat{\phi}^{\log }: \widehat{Y}^{\log } \rightarrow \widehat{X}^{\text {log }}$ (of $\mathbb{Z} / p^{2} \mathbb{Z}$-flat schemes). Then, by restricting $\widehat{\phi}^{\log }$ to the open subscheme of $\widehat{Y}^{\log }$ defined by the ordinary locus of $Z^{\prime \prime}$, we obtain a Frobenius lifting $\Xi^{\log }$ on the ordinary locus of $\widehat{X}^{\text {log }}$. The only points at which $\Xi$ is not defined are the supersingular points (determined by $\alpha$ ). Moreover, by the calculation of the discussion preceding Proposition 4.3, it follows that the local height of $\Xi^{\log }$ at a supersingular point is $\leq 1$. Indeed, in the notation of loc. cit., " $\pi$ " is equal to $p$ times an element of $k[[\vec{\xi}, \vec{\eta}]]$, which, this time, might not be a unit since $\widehat{Y}^{\mathrm{log}}$ might not be the reduction modulo $p^{2}$ of a regular scheme; hence the inequality $\leq 1$, rather than the sharp equality $=1$. At any rate, it thus follows that ht $(\Xi) \leq \mathrm{ht}\left(\Phi_{\mathrm{Z}_{/ p 2} \mathrm{Z}}\right)$. But then. by Proposition $4.10, \widehat{X}^{\log }$ is equal to some canonical curve reduced modulo $p^{2}$, and, by Proposition 2.6, (4), of Chapter II, it thus follows that this canonical curve is the one determined by $\alpha$. Thus, $\widehat{X}^{\log }=X_{2}^{l_{p 2} 2}$. But this means that $\mu=0$, so $X^{\text {log }}$ and $\widetilde{X}^{\text {log }}$ coincide modulo $p^{n+1}$. By Lemma 4.15 (the rigidity of an admissible Frobenius lifting). it thus follows that $\Phi^{\log }$ and $\left(\Phi^{\log }\right)^{\prime}$ also coincide modulo $p^{n+1}$. This proves the induction step, and hence the Lemma.

Putting everything together, we see that we have proven the following geometric criterion for a curve to be canonical:

Theorem 4.17. Let $X^{\log } \rightarrow S^{\log }$ be a smooth r-pointed curve of genus $g$. Let $\alpha$ be a $p$-adic quasiconformal equivalence to which $X^{\log }$ belongs. Then $X^{\log }$ is canonical if and omly if $\left(X^{\mathrm{log}}, \alpha\right)$ admits an admissible Frobenius lifting. 
Proof. We saw in $\S 3$ that a canonical curve admits an admissible Frobenius lifting. On the other hand, given an $\left(X^{\log }, \alpha\right)$ which admits an admissible Frobenius lifting, there exists an $\left(\widetilde{X}^{\text {log }}, \alpha\right)$ (with $\widetilde{X}^{\log } \equiv X^{\log }$ modulo $p$ ) which is canonical, hence admits an admissible Frobenius lifting. Thus, by Lemma 4.16, it follows that $X^{\mathrm{log}}=\widetilde{X}^{\mathrm{log}}$.

Definition 4.18. Suppose that $\left(X^{\log }, \alpha\right)$ admits an admissible Frobenius lifting $\Phi^{\log }$. Then we shall call $\Phi^{\log }$ the $p$-adic Green's function.

Remark. Then justification for this terminology is as follows. In the classical complex case, one of the main approaches to proving that hyperbolic curves can be uniformized by the upper half plane is given by constructing a Green's function on the universal covering space of the Riemann surface (see, e.g., $[\mathrm{FK}]$ ). Once one proves that the universal covering space is just the upper half plane. then one sees that this Green's function is really just the logarithm of the hyperbolic distance between two points. On the other hand, the canonical Frobenius lifting $\Phi^{\log }$ may also be regarded as giving us a notion of distance on $X^{\log }$. Indeed, in the classical modular case, where $X^{\log }$ parametrizes elliptic curves, if one can get from point $a$ to point $b$ by applying $\Phi^{10 g}$ a total of $N$ times, then it means that the corresponding elliptic curves are isogenous via a cyclic isogeny of order $p^{N}$. Thus, the analogy between $\Phi^{\log }$ and the classical complex Green's function (which is just the logarithm of the hyperbolic distance) will be established once one accepts that isogeny is the proper analogue of distance. But to see this, one need merely think of lattices in $\mathbb{Q}_{p}^{2}$. which one can draw schematically as a graph. Then two lattices are related by an isogeny of order $p^{N}$ if and only if they are $N$ edges apart on this graph. This establishes the relationship between isogeny and distance.

Remark. We also observe that for elliptic curves (regarded parabolically), the same definition of compactified Frobenius liftings, and admissible Frobenius liftings goes through, but everything is trivial, since there are no supersingular points to contend with. Thus, we (trivially) obtain the analogue of Theorem 4.17: that an elliptic curve over $A$ with ordinary reduction is canonical if and only if it admits a Frobenius lifting defined everywhere on the curve. 


\section{Chapter $\mathbb{V}$ : Uniformizations of Ordinary Curves}

\section{Introduction}

Having studied the case of canonical curves in the previous Chapter, in this Chapter we turn to the case of arbitrary curves with ordinary reduction modulo $p$. We do this by working with the universal case: i.e., the universal curve over the moduli stack. Unlike the canonical case, one does not quite obtain such objects as the canonical Galois representation or the canonical log p-divisible group over the given base. Instead, one must pass to various "schemes of multiplicative periods" - i.e., certain infinite coverings of the original base - in order to obtain such objects. On the other hand, since these objects are canonically associated to the curve over the given base, it is natural to guess that they should descend from the scheme of multiplicative periods back down to the original base in some appropriate sense. The key idea here is that, for instance in the case of the canonical Galois representation (which is fundamental to the construction of all the other objects), if one works with modules of rank two, not over $\mathbb{Z}_{p}$, but over some appropriate ring of $p$-adic periods $\mathscr{D}^{\text {Gal }}$, then one can in fact construct a canonical Galois representation over the original base. Thus, one obtains a representation of the entire arithmetic fundamental group into $\mathrm{GL}_{2}^{ \pm}\left(\mathscr{D}^{\mathrm{Gal}}\right)$, which in some sense extends the representation of the geometric fundamental group into $\mathrm{GL}_{2}^{ \pm}\left(\mathbb{Z}_{p}\right)$. Moreover, (in the hyperbolic case) this representation of the arithmetic fundamental group into $\mathrm{GL}_{2}^{ \pm}\left(\mathscr{D}^{\mathrm{Gal}}\right)$ is canonical, and dual crystalline in some appropriate sense, despite the fact that (unlike the case handled in [Falt], §2), it is on a space of infinite rank over $\mathbb{Z}_{p}$. The process of passing from the canonical representation of the geometric fundamental group into $G L^{\frac{ \pm}{2}}\left(\mathbb{Z}_{p}\right)$ to the canonical representation of the arithmetic fundamental group into $\mathrm{GL}_{2}^{ \pm}\left(\mathscr{D}^{\mathrm{Gal}}\right)$ is a sort of crystalline analogue of the notion of an induced representation in group theory. We therefore refer to this process as the process of crystalline induction.

Once one has this canonical dual crystalline representation of the arithmetic fundamental group into $\mathrm{GL}_{2}^{ \pm}\left(\mathscr{D}^{\mathrm{Gal}}\right)$, one can linearize the obstruction to extending the representation of the geometric fundamental group into $\mathrm{GL}_{2}^{ \pm}\left(\mathbb{Z}_{p}\right)$ to the full arithmetic fundamental group. This linearization tells one, for instance, that as soon as one can extend the representation of the geometric fundamental group into $\mathrm{GL}_{2}^{ \pm}\left(\mathbb{Z}_{p}\right)$ at all to the arithmetic fundamental group, this extension is automatically dual crystalline. This procedure of linearizing the obstruction also 
allows one to see that this obstruction is precisely the hyperbolic analogue of the obstruction to splitting a certain exact sequence of $p$-adic local systems on the moduli stack of ordinary elliptic curves (in the parabolic case).

\section{§1. Crystalline Induction}

In Chapter III, we constructed a Frobenius-invariant indigenous bundle on the universal curve over $\overline{\mathcal{N}}_{\boldsymbol{g}, r}^{\text {ord }}$. Unfortunately, unlike the case of a canonical curve, such information does not immediately constitute an object of the category $\mathcal{M} \mathscr{F}^{\nabla}$ (see [Falt], §2). so we cannot immediately convert it into a Galois representation. The problem is that our connection on the indigenous bundle is only a relative connection (for the universal curve over $\overline{\mathcal{N}}_{\boldsymbol{g}, r}^{\text {ord }}$ ), not a full connection on the total space of the universal curve. Also, the obstruction to extending it to a full connection on the total space of the universal curve is nonzero. Thus, in order to obtain a Galois representation, we must replace the indigenous bundle by a certain natural "thickening" of the indigenous bundle. This thickening formally carries the structure of an object of the category $M \mathscr{F}^{\nabla}$. but has the disadvantage of being of infinite rank, so that we cannot immediately apply the theory of [Falt] to this object. Fortunately, it is not difficult to extend the theory of [Falt] so as to handle such objects of infinite rank. We thus obtain a Galois representation, as desired, which turns out to be a sort of crystalline analogue of the notion of an "induced representation" in group theory.

\section{The Crystalline-Induced $M \mathscr{F}^{\nabla}$-object}

Let $p$ be an odd prime. Let $S$ be formally smooth over $A \stackrel{\text { def }}{=} W(k)$. where $k$ is a perfect field of characteristic $p$. Let us assume that $S$ is endowed with a $\log$ structure induced by a relative divisor with normal crossings over $W(k)$. Let $S^{\log }$ be the resulting log formal scheme. Let $f^{\log }: X^{\log } \rightarrow S^{\log }$ be an $r$-pointed stable curve of genus $g$. Also, let us assume that the classifying morphism $S \rightarrow \bar{M}_{g, r}$ defined by $f^{\log }$ is étale. Let $\Phi^{\log }: S^{\log } \rightarrow S^{\log }$ be an ordinary Frobenius lifting.

Now let us suppose that our indigenous bundle $\left(\mathscr{E}, \nabla_{\mathscr{E}}\right)$ on $X^{\mathrm{log}}$ is invariant under the renormalized Frobenius (Chapter III, Definition 1.4): that is,

$$
\Phi_{\varepsilon}:\left(\mathscr{E}, \nabla_{\mathscr{g}}\right) \cong \mathbb{F}_{S^{\mathrm{log}}}^{*}\left(\mathscr{E}, \nabla_{\mathscr{E}}\right)^{\Phi}
$$

where the superscript " $\Phi$ " denotes pull-back by $\Phi$, and the subscript " $S$ log" denotes that we are considering the relative renormalized Frobenius pull-back over $S^{\text {log }}$. (We shall denote by $\mathbb{F}_{A}^{*}$ the renormalized Frobenius pull-back over 
A.)

Let $\mathscr{D}_{S}$ be the quasi-coherent $\mathscr{O}_{S}$-algebra (with the $\mathscr{O}_{S}$-action from the right) which is obtained by taking the $p$-adic completion of the PD-envelope of the diagonal embedding of $S^{\log }$ in $S^{\log } \times{ }_{A} S^{\log }$ (see, e.g., [Kato], §5.8). Thus, $\mathscr{D}_{S}$ has an ideal $\mathscr{I}_{S} \subseteq \mathscr{D}_{S}$ with $\mathscr{D}_{S} / \mathscr{I}_{S}=\mathscr{O}_{S}$, and $\mathscr{I}_{S} / \mathscr{I}_{S}^{2} \cong \Omega_{S / A}^{\log }=\Omega_{S}^{\text {iog }}$. Note that $\mathscr{D}_{S}$ has a natural logarithmic connection $\nabla_{\mathscr{D}_{S}}$. We shall regard $\mathscr{D}_{S}$ as a filtered object with connection, whose filtration is given by $F^{1}\left(\mathscr{D}_{S}\right)=\mathscr{I}_{S}^{[]]}$(i.e., divided powers of $\mathscr{I}_{S}$ ). Thus, the Kodaira-Spencer morphism for the subquotient of the filtration given by $F^{1}\left(\mathscr{D}_{S}\right) / F^{2}\left(\mathscr{D}_{S}\right)=\mathscr{I}_{S} / \mathscr{I}_{S}^{2} \cong \Omega_{S}^{\log }$ is the identity map. Note that the Frobenius lifting $\Phi^{\text {log }}$ on $S^{\text {log }}$ induces a morphism $\Phi_{\mathscr{D}_{S}}: \Phi^{*} \mathscr{D}_{S} \rightarrow \mathscr{D}_{S}$ which preserves the Hodge filtration. Finally, let us denote by $\left(\left(\mathscr{L}_{S}\right)_{X}, \nabla\left(\mathscr{D}_{S}\right)_{X}\right)$ the pull-back of $\left(\mathscr{D}_{S}, \nabla_{\mathscr{D}_{S}}\right)$ to $X^{\mathrm{log}}$

Next, let us consider the obstruction to defining a full logarthmic connection $\nabla$ on $\mathscr{E}$ (i.e., relative to $X^{\log } \rightarrow \operatorname{Spec}(A)$ ) with the following properties:

(1) $\nabla$ has trivial determinant;

(2) the restriction of $\nabla$ to a relative connection (for $X^{\log } \rightarrow S^{\log }$ ) is $\nabla_{\mathscr{E}}$

(3) the curvature of $\nabla$ is an $\operatorname{Ad}(\mathscr{E})$-valued section of $\wedge^{2} \Omega_{S}^{\log }$ (i.e., the $\omega_{X / S}^{\log } \otimes_{O_{S}} \Omega_{S}^{\mathrm{log}}$-part of the curvature vanishes).

It is easy to see that the obstruction class to defining such a connection is a section $\eta_{\mathscr{E}}$ of

$$
\mathbb{R}^{1} f_{\mathrm{DR}, *} \operatorname{Ad}(\mathscr{E}) \otimes_{\mathbb{O}_{S}} \Omega_{S}^{\log }=F^{0}\left(\mathbb{R}^{1} f_{\mathrm{DR}, *} \operatorname{Ad}(\mathscr{E}) \otimes_{\mathcal{O}_{S}} \Omega_{S}^{\log }\right)
$$

whose projection to $\mathbb{R}^{1} f_{*} \tau_{X^{100 /} / S^{\text {log }}} \otimes \mathcal{O}_{S} \Omega_{S}^{\log }$ is the identity. Also, note that $\Phi^{\text {log }}$ and $\Phi_{\mathscr{E}}$ induce a Frobenius action on $\mathbb{R}^{1} f_{\mathrm{DR}, *} \operatorname{Ad}(\mathscr{E}) \otimes_{\mathcal{O}_{S}} \Omega_{S}^{\log }$, which, by naturality, preserves $\eta_{\mathscr{E}}$. Thus, in particular, we see that unless we modify $\mathscr{E}$ in some way, there is no hope of constructing a full connection $\nabla$ as specified above.

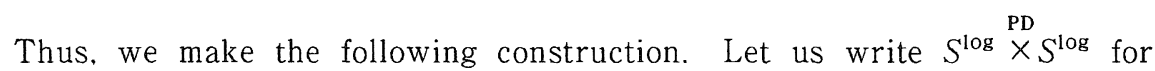
$\operatorname{Spf}\left(\mathscr{D}_{S}\right)$ (where we take "Spf" with respect to the $p$-adic topology). Similarly, we shall write $S^{\log } \stackrel{\text { PD }}{\times} S^{\log } \stackrel{\text { PD }}{\times} S^{\log }, X^{\log } \stackrel{\text { PD }}{\times} X^{\log }$. etc. for the obvious $p$-adic completions of $\mathrm{PD}$-envelopes at the respective diagonals. Also, we have two projections $\pi_{\mathrm{R}}, \pi_{\mathrm{L}}: S^{\log } \stackrel{\text { PD }}{\times} S^{\log } \rightarrow S^{\log }$ to the left and right by which we can pull-back

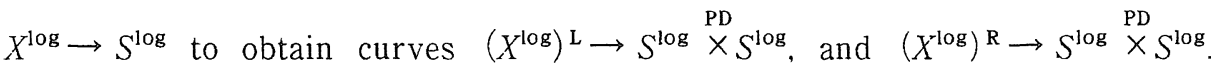
Moreover, both of these curves form PD-thickenings of $X^{\log } \rightarrow S^{\log } \hookrightarrow S^{\log } \times S^{\log }$ (where the second morphism is the diagonal embedding). It thus follows that if we pull-back $\left(\mathscr{E}, \nabla_{\S}\right)$ to obtain an indigenous bundle on the curve 
$\left(X^{\log }\right)^{\mathrm{L}} \rightarrow S^{\log } \times S^{\mathrm{PD}}$, this indigenous bundle defines a crystal on Crys $\left(X^{\log } /\left(S^{\log } \times S^{\log }\right)\right)$ which we can then evaluate on the thickening $\left(X^{\log }\right)^{\mathrm{R}}$ to obtain a rank two vector bundle $\mathscr{E}^{\prime}\left(\right.$ on $\left.X^{\mathrm{R}}\right)$. If we then push this sheaf $\mathscr{E}^{\prime}$ forward via the projection $X^{\mathrm{R}} \rightarrow X$, we obtain a quasi-coherent sheaf $\mathscr{E} \mathscr{D}$ on $X$. Moreover, $\mathscr{E} \mathscr{D}$ has the structure of a $\mathscr{D}_{S}$-module, hence of a $\left(\mathscr{D}_{S}\right)_{X}$-module. In fact, $\mathscr{E} \mathscr{D}$ is a locally free $\left(\mathscr{D}_{S}\right)_{X}$-module of rank two. Moreover, $\mathscr{E} \mathscr{D}$ is equipped with a natural Hodge filtration compatible with that of $\left(\mathscr{D}_{S}\right)_{X}$.

Next, we would like to equip $\mathscr{E} \mathscr{D}$ with a full logarithmic connection that is compatible with its structure as a $\left(\mathscr{D}_{S}\right)_{X}$-module and the connection on $\left(\mathscr{D}_{S}\right)_{X}$. First, note that $X^{\log } \stackrel{\text { PD }}{\times} S^{\log } \stackrel{\text { PD }}{\times} S^{\log } \rightarrow S^{\log } \stackrel{\text { PD }}{\times} S^{\log } \stackrel{\text { PD }}{\times} S^{\log }$ is a PD-thickening of $X^{\log } \rightarrow$ $S^{\log } \hookrightarrow S^{\log } \times S^{\operatorname{PD}} \stackrel{\text { PD }}{\times} S^{\log }$ (where the second morphism is the diagonal embedding).

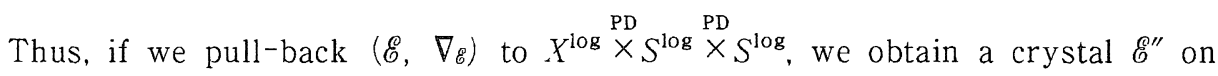
Crys $\left(X^{\log } /\left(S^{\log } \times \stackrel{P D}{\times} S^{\log } \times S^{\operatorname{PD}}\right)\right)$. On the other hand, $S^{\log } \stackrel{\text { PD }}{\times} X^{\log } \stackrel{\text { PD }}{\times} X^{\log } \rightarrow S^{\log } \times S^{\operatorname{PD}}$ $\stackrel{\text { PD }}{\times} S^{\log }$ is also a PD-thickening of $X^{\log } \rightarrow S^{\log } \hookrightarrow S^{\log } \stackrel{\text { PD }}{\times} S^{\log } \stackrel{\text { PD }}{\times} S^{\log }$. Thus, if we evaluate $\mathscr{E}^{\prime \prime}$ on $S^{\log } \stackrel{\text { PD }}{\times} X^{\log } \stackrel{\text { PD }}{\times} X^{\log }$, and then push forward via the projection

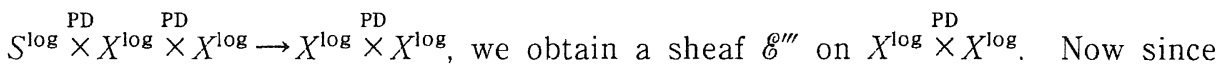
$\left(\mathscr{D}_{S}\right)_{X}$ is equipped with a connection, the two pull-backs of $\left(\mathscr{D}_{S}\right)_{X}$ to $X^{\log } \times X^{\text {log }}$ via the two projections $\pi_{\mathrm{R}}^{X}, \pi_{\mathrm{L}}^{X}: X^{\log } \times X^{\mathrm{PD}} \rightarrow X^{\log }$ can be identified; we denote the

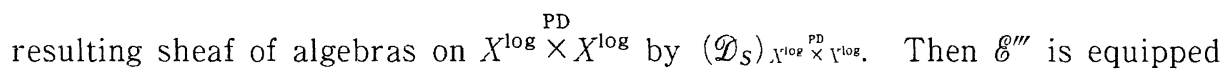

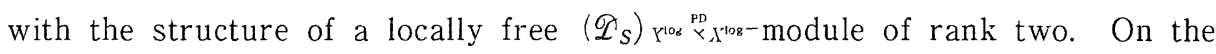
other hand, from the definition of $\mathscr{E} \mathscr{E}$. it follows that both $\left(\pi_{\mathrm{L}}^{X}\right) * \mathscr{E} \mathscr{D}$ and $\left(\pi_{\mathrm{R}}^{X}\right) * \mathscr{E} \mathscr{D}$

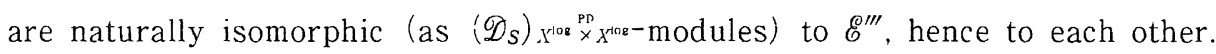

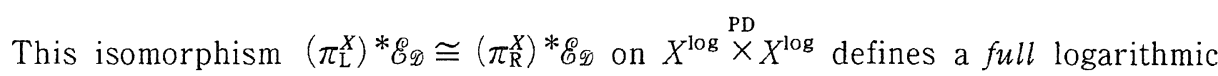
connection $\nabla_{\mathscr{g} \mathscr{D}}$ on $\mathscr{E} \mathscr{D}$ (with respect to $\left.X^{\log } \rightarrow \operatorname{Spec}(A)\right)$. Moreover, one checks easily that this connection is integral.

Finally, we have a Frobenius action

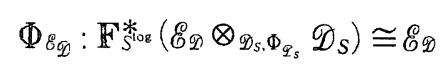

Here, in the definition of $\mathbb{F}_{S^{\mathrm{iog}}}^{*}$, we first pull back the relevant crystal by means of relative Frobenius, and then consider the subsheaf consisting of sections whose reduction modulo $p$ is contained in the subsheaf of

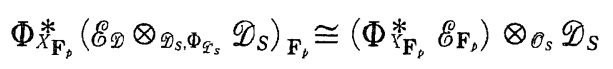


given by

$$
\left(\bar{\Phi}_{X_{F_{p}}}^{*} F^{1}(\mathscr{E})_{\mathbf{F}_{p}}\right) \otimes_{\mathscr{O}_{s}} \mathscr{D}_{S} \subseteq\left(\bar{\Phi}_{X_{F_{p}}}^{*} \mathscr{E} \mathbf{F}_{p}\right) \otimes_{\mathscr{O}_{S}} \mathscr{D}_{S}
$$

Theorem 1.1. Over $X^{\mathrm{log}}$, there exists a natural. locally free, rank two $\left(\mathscr{D}_{S}\right)_{X^{-}}$ module $\mathscr{E}_{D}$ equipped with a Hodge filtration, a full integrable logarithmic connection $\nabla_{\mathscr{g}_{\mathscr{D}}}\left(\right.$ relative to $\left.X^{\mathrm{log}} \rightarrow \operatorname{Spec}(A)\right)$, and a Frobenius action

$$
\bar{\Phi}_{\mathscr{B}_{\mathscr{D}}}: \mathbb{F}_{\mathcal{S}}^{*}\left(\mathscr{E}_{\mathscr{D}} \otimes_{\mathscr{D}_{S}, \Phi_{\Phi_{S}}} \mathscr{D}_{S}\right) \cong \mathscr{E} \mathscr{D}
$$

such that $\mathscr{E} D \otimes_{\mathscr{D}_{S}}\left(\mathscr{D}_{S} / \mathscr{I}_{S}\right)=\mathscr{E}$.

\section{The Ring of Additive Periods}

Before we can convert the induced object of Theorem 1.1 into a Galois representation, we must first study the Galois representation associated to $\mathscr{D}_{S}$, with its natural filtration, connection, and Frobenius action. Once we have done this, since $\mathscr{E} \mathscr{D}$ is of finite rank over $\mathscr{D}_{S}$, converting $\mathscr{E} \mathscr{D}$ into a Galois representation will be no more difficult than the "classical case" discussed in [Falt], §2.

Let us first note that, just as when we constructed "canonical affine coordinates" in Chapter III. $\S 1$, by considering the slopes of the Frobenius action $\Phi_{\mathscr{D}_{s}}$, we obtain a unique Frobenius-equivariant embedding of $\mathscr{O}_{S}$-modules

$$
\Omega_{S}^{\log C} \mathscr{I}_{S}
$$

whose composite with the projection $\mathscr{I}_{S} \rightarrow \mathscr{I}_{S} / \mathscr{I}_{S}^{2}=\Omega_{S}^{\text {log }}$ is the identity. It is here that we use the divided powers of $\mathscr{I}_{S} \subseteq \mathscr{D}_{S}$. Let us write Geo $\left(\mathscr{D}_{S}\right)$ for the subbundle of $\mathscr{D}_{S}$ generated by $\Omega_{S}^{\log }$ and $\mathscr{O}_{S}$. Note that $\mathrm{Geo}\left(\mathscr{D}_{S}\right)$ is stabilized by $\nabla_{\mathscr{D}_{S}}$ and by Frobenius. Moreover. the Hodge filtration on $\mathscr{D}_{S}$ induces a Hodge filtration on $\mathrm{Geo}\left(\mathscr{D}_{S}\right)$. Observe that with this extra data, Geo $\left(\mathscr{D}_{S}\right)$ becomes isomorphic to the uniformizing $\mathcal{M} \mathscr{F}^{\nabla}$-object associated to $\Phi^{\log }$ (of Definition 1.3 of Chapter III).

Now we want to pass to Galois representations. Let us assume that we have chosen (once and for all) a base-point of $S$ that avoids the divisors defining the log structure. In the following, our fundamental groups will be with respect to this base-point. Since our construction will be canonical, we can work étale locally on $S$. Thus, we can assume that $S$ is affine. We may also assume that $S^{\log }$ is small (in the sense of [Falt], §2): that is, $S^{\log }$ is $\log$ étale over $A\left[T_{1}, \ldots, T_{d}\right]$ (with the $\log$ structure given by the divisor $T_{1}{ }^{\circ} \ldots{ }^{\circ} T_{d}$ ). We shall 
call these parameters $T_{1}, \ldots, T_{d}$ small parameters. Then we would like to consider the ring $B^{+}\left(S^{\text {log }}\right)$ of $[$ Falt $], \S 2$. We will not review the definition of this ring here, since it is rather involved, but roughly speaking, it is obtained by

(1) taking the normalization $\widetilde{S}$ of $S^{\log }$ in the maximal covering of $S_{K}^{\log }$ which is étale in characteristic zero;

(2) reducing $\widetilde{S}$ modulo $p$ and taking its perfection;

(3) taking the Witt ring with coefficients in this perfection;

(4) adjoining the divided powers of a certain ideal to this Witt ring; and

(5) finally, completing with respect to a certain topology. In particular,

(1) $B^{+}\left(S^{\log }\right)$ is obtained as the inverse limit of a projective system of PD-thickenings of the $\mathcal{O}_{S_{F_{p}}}$-algebra $\widetilde{S}_{F_{p}}$;

(2) $B^{+}\left(S^{\log }\right)$ has an ideal $I^{+} \subseteq B^{+}\left(S^{\log }\right)$ which is Galois-invariant and such that $B^{+}\left(S^{\log }\right) / I^{+} \cong \widetilde{S}^{\wedge}$ (i.e., the $p$-adic completion of $\widetilde{S}$ ).

Moreover, $B^{+}\left(S^{10 g}\right)$ comes equipped with a natural Frobenius action (which we shall denote by means of a superscripted " $F$ "), as well as a continuous $\pi_{1}\left(S_{K}^{\log }\right)$ -action, which commutes with the Frobenius. The Frobenius invariants of $B^{+}\left(S^{\log }\right)$ are given by $\mathbb{Z}_{p} \subseteq B^{+}\left(S^{\log }\right)$. There is a Galois equivariant injection $\beta: \mathbb{Z}_{p}(1) \hookrightarrow B^{+}\left(S^{\log }\right)$. Frequently, we shall abuse notation and write $\beta \in B^{+}\left(S^{\log }\right)$ for the element of $B^{+}\left(S^{\log }\right)$ obtained by applying $\beta$ to some generator of $\mathbb{Z}_{p}(1)$. Then the Frobenius action on $\beta$ takes $\beta$ to $p \cdot \beta$. We will denote by $B\left(S^{\log }\right)$ the ring obtained from $B^{+}\left(S^{\log }\right)$ by inverting $\beta$ and $p$. This completes our review of $B^{+}(-)$.

Now let us return to the specific situation we have at hand. By thinking of $\left(\mathscr{D}_{S}, \nabla_{\mathscr{D}_{S}}\right)$ as a crystal, and using the fact that $B^{+}\left(S^{\log }\right)$ is an inverse limit of PD-thickenings of a certain $\mathscr{O}_{S_{F_{p}}}$-algebra, one can evaluate this crystal on $B^{+}\left(S^{\log }\right)$ (and complete $p$-adically) to obtain a $B^{+}\left(S^{\log }\right)$-module which we shall denote by

$$
\mathscr{D}_{S} \widehat{\otimes}_{C_{S}} B^{+}\left(S^{\log }\right)
$$

(where the "hat" denotes $p$-adic completion). Alternatively, one can embed $\mathscr{O}_{S}$ into $B^{+}\left(S^{\log }\right)$ by means of a choice of small parameters, and then take the literal tensor product, as described in $\left[\mathrm{Falt}_{\lrcorner}\right], \S 2$. In our situation, however, since we are given a Frobenius lifting $\Phi^{\text {log }}$, the most useful point of view will be to embed

$$
\mathscr{O}_{S} \hookrightarrow B^{+}\left(S^{\log }\right)
$$


$\mathcal{O}_{S}$ into $B^{+}\left(S^{\log }\right)$ by means of the Frobenius lifting $\Phi^{\log }$. Indeed, the choice of Frobenius lifting gives us an embedding of $\mathscr{O}_{S}$ into the ring of Witt vectors that appears in the construction (reviewed above) of $B^{+}\left(S^{\log }\right)$. Then, we may regard the module considered above as obtained via the literal tensor product with respect to this particular embedding of $\mathscr{O}_{S}$ into $B^{+}\left(S^{\log }\right)$. At any rate, $\mathscr{D}_{S}$ $\widehat{\otimes}_{\mathscr{O}_{s}} B^{+}\left(S^{\log }\right)$ has a natural filtration and Frobenius action. Let $T^{\log } \rightarrow S^{\log }$ be the finite covering defined by $\Phi^{\log }$. (Thus. $T^{\log } \cong S^{\log }$.) Then $\mathscr{D}_{S} \widehat{\otimes}_{\mathscr{C}_{s}} B^{+}\left(S^{\log }\right)$ also has a natural action by $\pi_{1}\left(T_{K}^{\mathrm{log}}\right)$. The reason why we must restrict to $T^{\text {log }}$, rather than considering all of $\pi_{1}\left(S_{K}^{\text {log }}\right)$, is that the way the Galois action is defined (see [Falt]. §2) involves exponentiating the connection $\nabla_{\mathscr{y}_{s}}$, so in order for the exponential series to converge, one must be in a situation where the connection acts in a sufficiently nilpotent fashion. For convenience, let us write $\Pi_{T^{\text {Iog }}}$ (respectively, $\left.\Pi_{S^{\text {Iog }}}\right)$ for $\pi_{1}\left(T_{K}^{\text {log }}\right)$ (respectively, $\pi_{1}\left(S_{K}^{\text {log }}\right)$ ).

Let us recall the uniformizing Galois representation $\mathscr{P}_{\mathrm{et}}$ (Definition 1.4 of Chapter III) associated to the ordinary Frobenius lifting $\Phi^{\log }$. Recall that $\mathscr{P}_{\text {et }}$ fits into an exact sequence of $\mathbb{\Pi}_{T^{108}-\text { modules }}$

$$
0 \rightarrow \Theta_{\Phi}^{\mathrm{el}}(1) \rightarrow \mathscr{P}_{\mathrm{et}} \rightarrow \mathbb{Z}_{p} \rightarrow 0
$$

The space of splittings of this sequence then forms an affine $\mathbb{Z}_{p}$-scheme, which is the spectrum of some ring $\operatorname{Aff}\left(\mathscr{P}_{\mathrm{et}}^{\mathrm{V}}\right)$. More concretely. $\operatorname{Aff}\left(\mathscr{P}_{\mathrm{et}}^{\mathrm{V}}\right)$ is a polynomial ring over $\mathbb{Z}_{p}$ in $3 g-3+r$ variables which is equipped with an action by $\Pi_{T^{\text {or }}}$. Moreover, the submodule of polynomials of degree $\leq 1$ is given by $\mathscr{P} \mathrm{et}^{\vee} \hookrightarrow$ $\operatorname{Aff}\left(\mathscr{P}_{\mathrm{et}}^{\mathrm{e}}\right)$. We shall refer to $\operatorname{Aff}\left(\mathscr{P}_{\mathrm{et}}^{\vee}\right)$ as the affinization of $\mathscr{P}_{\mathrm{et}} \mathrm{V}$.

Note that $\operatorname{Aff}\left(\mathscr{P}_{\mathrm{et}}\right)$ has a natural $\prod_{T^{\text {log }}}$-invariant augmentation $\operatorname{Aff}\left(\mathscr{P} V_{\mathrm{et}}\right) \rightarrow$ $\mathbb{F}_{p}$. Let $\mathscr{D}_{S}^{\text {Gal }}$ be the $p$-adic completion of the $\mathrm{PD}$-envelope of $\operatorname{Aff}\left(\mathscr{P}_{\mathrm{et}}^{\vee}\right)$ at this augmentation. Thus, $\mathscr{D}_{S}^{\mathrm{Gal}}$ is equipped with a natural structure of $\Pi_{T^{\text {jog }} \text {-algebra, }}$, and, moreover, we have a $\Pi_{T^{100-i n v a r i a n t} \text { inclusion }}$

$$
\mathscr{P} \vee \mathrm{et}
$$

In other words, $\mathscr{D}_{S}^{\mathrm{Gal}}$ may be identified with the ring of additive periods (of Definition 1.5 of Chapter III). On the other hand, since $\mathscr{P}_{\text {et }}$ is the Galois representation contravariantly associated to Geo $\left(\mathscr{D}_{S}\right)$, it follows that we have a morphism

$$
\mathscr{P}_{\mathrm{et}} \rightarrow \mathrm{Geo}\left(\mathscr{D}_{S}\right) \vee \widehat{\otimes}_{\mathscr{O}_{S}} B^{+}\left(S^{\log }\right)
$$

which respects the Hodge filtrations, Frobenius actions (where $\mathscr{P}_{\eta}$ is endowed with the trivial Hodge filtration and Frobenius action), and Galois actions (by $\left.\Pi_{T^{108}}\right)$. "Switching duals," we thus see that we have a morphism 


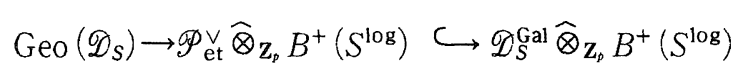

which respects Hodge, Frobenius, and Galois. Next, since $\mathrm{Geo}\left(\mathscr{D}_{S}\right)$ generates $\mathscr{D}_{S}$ as a "PD-polynomial algebra" with no relations, it thus follows that we obtain a morphism

$$
\mathscr{D}_{S} \rightarrow \mathscr{D}_{S}^{\text {Gal }} \widehat{\otimes}_{\mathrm{Z}_{p}} B^{+}\left(S^{\log }\right)
$$

which respects Hodge, Frobenius, and Galois. Finally, tensoring with $B^{+}\left(S^{\log }\right)$, we obtain the following result:

Proposition 1.2. We have a morphism

$$
\mathscr{D}_{S} \widehat{\otimes}_{\widehat{O}_{S}} B^{+}\left(S^{\log }\right) \rightarrow \mathscr{D}_{S}^{\mathrm{Gal}} \widehat{\otimes}_{\mathrm{Z}_{p}} B^{+}\left(S^{\log }\right)
$$

which respects Hodge filtrations, Frobenints actions, and Galois actions (by $\Pi_{T^{108}}$ ).

\section{The Crystalline-Induced Galois Representation}

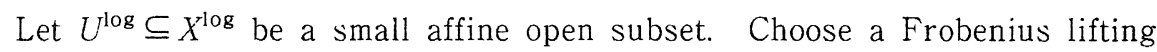
$\Psi^{\log }$ on $U^{\log }$ that is compatible with $\Phi^{\log }$ on $S^{\log }$. Thus, $\Psi^{\log }$ gives us an embedding of $\mathscr{O}_{U}$ into $B^{+}\left(U^{\text {log }}\right)$ which fits into a commutative diagram

$$
\begin{array}{ccc}
\mathscr{O}_{S} & \rightarrow & B^{+}\left(S^{\log }\right) \\
\downarrow & & \downarrow \\
\mathscr{O}_{U} & \rightarrow & B^{+}\left(U^{\log }\right) .
\end{array}
$$

We would like to consider

$$
\mathscr{G}_{U} \stackrel{\text { def }}{=} \mathscr{\mathscr { E }} \vee \widehat{\mathscr{Q}}_{\widehat{C}_{U}} B^{+}\left(U^{\mathrm{log}}\right)
$$

where the " $\vee$ " denotes the dual as a $\mathscr{D}_{S}$-module. The problem is to show that $\mathscr{G}_{U}$ has enough Frobenius-invariant sections in the zeroth step of its filtration. The reason that we cannot apply the theory of [Falt], $\$ 2$ directly is that the relevant theorem (Theorem 2.4 of loc. cit.) assumes a bound on the number of steps in the filtration of the $\mathcal{M} \mathscr{F}^{\nabla}$-object under consideration. On the other hand, $\mathscr{E} D$ has infinitely many steps.

What we can do is base-change $\mathscr{G}_{U}$ by the morphism of Proposition 1.2. We then obtain a free $\left(\mathscr{D}_{S}^{\text {Gal }} \widehat{\otimes}_{\mathbf{Z}_{p}} B^{+}\left(U^{\text {log }}\right)\right)$-module 


$$
\mathscr{G}_{U}^{\prime}
$$

of rank two. Moreover, $\mathscr{G}_{U}^{\prime}$ is equipped with a Galois action, a Hodge filtration, and a Frobenius action

$$
\mathbb{F}^{*}\left(\left(\mathscr{G}_{U}^{\prime}\right)^{F}\right) \cong \mathscr{G}_{U}^{\prime}
$$

(where the superscripted " $F$ " denotes base-change by the Frobenius on $\left.B^{+}\left(U^{\mathrm{log}}\right)\right)$.

Proposition 1.3.The submodule

$$
\left(F^{0}\left(\mathscr{G}_{U}^{\prime}\right)\right)^{F=1}
$$

(consisting of Frobenius-invariant elements of $F^{0}\left(\mathscr{G}_{U}^{\prime}\right)$ ) forms a free $\mathscr{D}_{S}^{\mathrm{Gal}}$-module $E_{U}^{\mathrm{Gal}}$ of rank two.

Proof. The proof is entirely the same as that of Theorem 2.4 of [Falt], §2. The point of base-changing by the morphism of Proposition 1.2 is that this enables us to replace objects like $\mathscr{E} \vee$ whose Hodge filtrations have infinitely many steps by objects like $\mathscr{G}_{U}^{\prime}$ whose Hodge filtration has essentially only two steps. In fact, over $T^{\log }$. the relative connection $\nabla_{\mathscr{E}}$ on $\mathscr{E}$ actually extends to a full connection $\nabla^{\prime}$ modulo $p$, so $\left(\mathscr{E}_{F_{p}}, \nabla^{\prime}\right)$ defines a Galois representation onto some $\mathbb{F}_{p}$-vector space $E^{\prime}\left(\subseteq \mathscr{E} \vee \mathrm{F}_{b} \otimes B^{+}\left(U^{\mathrm{log}}\right)_{\mathrm{F}_{b}}\right)$ of dimension two. Moreover, $\mathscr{G}_{U}^{\prime}$ has a filtration (defined by taking divided powers of the augmentation ideal of $\mathscr{D}_{S}^{\mathrm{Gal}} \rightarrow$ $\left.\mathbb{F}_{p}\right)$ whose subquotients are tensor products of $\mathscr{E} \vee \mathrm{F}_{b} \otimes B^{+}\left(U^{\log }\right)_{\mathbf{F}_{b}}$ with symmetric powers of $\Omega^{\text {et }}(-1)_{\mathbf{F}_{p}}$. That is to say, we know that $F^{0}(-)^{F=1}$ for all of these subquotients is as desired, so next we want to consider the issue of whether the various extensions involved split over $B^{+}\left(U^{\log }\right)$. But this issue is precisely that discussed in the proof of Theorem 2.4 of [Falt], §2. Thus, we see that we have enough Frobenius invariants, at least over the PD-completion of $\mathscr{D}_{S}^{\text {Gal }}$. But it is a simple exercise to see that the fact that the original Frobenius action is defined over $\mathscr{D}_{S}^{\mathrm{Gal}}$ (i.e., not just over the PD-completion of $\mathscr{D}_{S}^{\mathrm{Gal}}$ ) implies that the Frobenius invariants will also be defined over $\mathscr{D}_{S}^{\text {Gal }}$ itself. This completes the proof.

Let $X_{T}^{\log } \stackrel{\text { def }}{=} X^{\log } \times_{S^{\log }} T^{\log }$. Similarly, we have $U_{T}^{\log } \subseteq X_{T}^{\text {log }}$. Now we have a natural $\pi_{1}\left(\left(U_{T}^{\mathrm{log}}\right)_{K}\right)$-action on $\mathscr{G}_{U}^{\prime}$. Since this action preserves the filtration and 
commutes with Frobenius, we thus get an action of $\pi_{1}\left(\left(U_{T}^{\log }\right)_{K}\right)$ on $E_{U}^{\mathrm{Gal}}$, which is

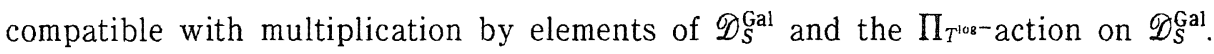
Moreover, as we vary the open subset $U \subseteq X$, the resulting $E_{U}^{\mathrm{Gal}}$ 's are clearly compatible. Thus, they glue together to form a $\pi_{1}\left(\left(X_{T}^{\mathrm{log}}\right)_{K}\right)-\mathscr{D}_{S}^{\mathrm{Gal}}-$ module

$$
E_{X}^{\mathrm{Gal}}
$$

We state this as a Theorem:

Theorem 1.4. The crystalline-induced $M \mathscr{F}^{\nabla_{-}}$object

$$
\left(\mathscr{E}_{D} ; F^{\imath}(\mathscr{E} \mathscr{D}) ; \Phi_{\mathscr{E}} ; \nabla_{\mathscr{E} \mathscr{D}}\right)
$$

of Theorem 1.1 has associated to it an $(u p$ to \pm 1$) \pi_{1}\left(\left(X_{T}^{\mathrm{log}}\right)_{K}\right)-\mathscr{D}_{S}^{\mathrm{Gal}}-$ module

$$
E_{X}^{\mathrm{Gal}}
$$

which is a free $\mathscr{D}_{S}^{\mathrm{Ga}}$-module of rank two. We shall refer to $E_{X}^{\mathrm{Gal}}$ as the crystallineinduced Galois representation associated to the induced $M \mathscr{F}^{\nabla}$-object of Theorem 1.1.

Remark. Unlike the case of canonical curves, where one actually has a dual crystalline representation (in the sense of [Falt], §2) into $\mathrm{GL}_{2}^{ \pm}\left(\mathbb{Z}_{p}\right)$, in the case of noncanonical curves, $E_{X}^{\mathrm{Gal}}$ is as close a $p$-adic analogue as one can get to the canonical representation in the complex case. In the following subsection, we shall make the phrase "as close as one can get" more precise.

\section{Relation to the Canonical Affine Coordinates}

Let $T_{\infty}^{\log } \rightarrow S^{\log }$ be the inverse limit of the coverings obtained by iterating $\Phi^{\log }$. Let $X_{T_{\infty}}^{\log } \rightarrow T_{\infty}^{\log }$ be the pull-back to $T_{\infty}^{\log }$ of $X^{\log } \rightarrow S^{\log }$. Let us choose base-points once and for all, and let

$$
\Pi_{1} \stackrel{\text { def }}{=} \pi_{1}\left(\left(X_{T}^{\log }\right)_{K}\right) ; \Pi_{\infty} \stackrel{\text { def }}{=} \pi_{1}\left(\left(X_{T_{\infty}}^{\log }\right)_{K}\right)
$$

Thus, $\Pi_{\infty} \subseteq \Pi_{1}$ is a closed subgroup. In the preceding subsection, we constructed a $\mathscr{D}_{S}^{\mathrm{Gal}}-\Pi_{1}-$ module which we called $E_{X}^{\mathrm{Gal}}$. If we restrict our Galois representations from $\Pi_{1}$ to $\Pi_{\infty}$, then we obtain a $\Pi_{\infty}$-equivariant surjection

$$
\pi_{S}: \mathscr{D}_{S}^{\mathrm{Gal}} \rightarrow \mathbb{Z}_{p}
$$


whose kernel is the augmentation ideal $\mathscr{g}_{S}^{\mathrm{Gal}}$, i.e., the PD-ideal generated by $\Omega_{S}^{\mathrm{Gal}}$. Thus, if we base change $E_{X}^{\mathrm{Gal}}$ by $\pi_{s}$, we obtain a rank two $\mathbb{Z}_{p}$-module with a continuous $\mathbb{\Pi}_{\infty}$-action which we denote by:

$$
E_{0} \stackrel{\text { def }}{=} E_{X}^{\mathrm{Gal}} \otimes_{\mathscr{D}_{S}^{\text {cal }}} \pi_{s} \mathbb{Z}_{p}
$$

Thus, in summary, $E_{0}$ has the advantage that it is of rank two over $\mathbb{Z}_{p}$, but the disadvantage that it only has a $\Pi_{\infty}$ - (as opposed to a full $\Pi_{1^{-}}$) action, while $E_{X}^{\mathrm{Gal}}$ has the advantage that is has a natural $\Pi_{1}$-action, but the disadvantage that it is of rank two over the rather large ring of additive periods $\mathscr{D}_{S}^{\mathrm{Gal}}$. In this subsection, we show that the canonical affine coordinates (of Chapter III, Theorem 3.6) measure precisely the degree to which the $\Pi_{\infty}$ action on $E_{0}$ cannot be extended to a full action of $\mathbb{\Pi}_{1}$.

We begin with the following fundamental observation. Let $\Delta \subseteq \Pi_{\infty} \subseteq \mathbb{\Pi}_{1}$ be the geometric fundamental group, i.e., the kernel of the natural morphism $\Pi_{1} \rightarrow$ $\pi_{1}\left(T_{K}^{\mathrm{log}}\right)$. Let us consider the exact sequence of $\Pi_{\infty}$-modules

$$
0 \rightarrow E_{0} \otimes_{\mathbf{Z}_{p}} \mathscr{J}_{S}^{\mathrm{Gal}} /\left(\mathscr{F}_{S}^{\mathrm{Gal}}\right)^{2} \rightarrow E_{X}^{\mathrm{Gal}} \otimes_{\mathscr{D}_{S}^{\mathrm{Gal}}} \mathscr{D}_{S}^{\mathrm{Gal}} /\left(\mathscr{F}_{S}^{\mathrm{Gal}}\right)^{2} \rightarrow E_{0} \rightarrow 0
$$

By considering the underlying $\Delta$-module structures, we obtain that the above exact sequence defines an extension class

$$
\eta^{\mathrm{Gal}} \in H^{1}\left(\Delta, \operatorname{Ad}\left(E_{0}\right)\right) \otimes_{\mathrm{Z}_{p}} \mathscr{g}_{S}^{\mathrm{Gal}} /\left(\mathscr{g}_{S}^{\mathrm{Gal}}\right)^{2}=\mathrm{H}^{1}\left(\Delta, \operatorname{Ad}\left(E_{0}\right)\right) \otimes_{\mathrm{Z}_{p}} \Omega_{S}^{\mathrm{Gal}}
$$

which is fixed by the natural action of $\pi_{1}\left(\left(T_{\infty}^{\log }\right)_{K}\right)$ on this cohomology group. On the other hand, because our original indigenous bundle is ordinary, we see that we have a $\pi_{1}\left(\left(T_{\infty}^{\log }\right)_{K}\right)$-equivariant inclusion

$$
\left(\Omega_{S}^{\mathrm{Gal}}\right) \vee \hookrightarrow H^{1}\left(\Delta, \operatorname{Ad}\left(E_{0}\right)\right)
$$

Then we claim that $\eta^{\mathrm{Gal}}$ is precisely the class is $\mathrm{H}^{1}\left(\Delta, \operatorname{Ad}\left(E_{0}\right)\right) \otimes_{\mathbf{Z}_{p}} \Omega_{S}^{\text {Gal }}$ that corresponds to this inclusion. Indeed, this follows immediately from observing that $\eta^{\text {Gal }}$ is essentially the Galois version of the class $\eta_{B}$ (the obstruction to the existence of a full connection on $\mathscr{E}$ ) that we encountered on our way to constructing $\mathscr{E} \mathscr{D}$. It then follows immediately from the way one passes from $\mathcal{M F}_{\mathbf{F}} \boldsymbol{D}_{-}$ objects to Galois representations that $\eta^{\text {Gal }}$ is the above inclusion, as claimed. This observation concerning $\eta^{\text {Gal }}$ will be the fundamental "hard fact" underlying what we do in this subsection; the rest will be general nonsense.

The general nonsense that we will use is the theory of [Schl]. Let us de- 
note the $\Pi_{\infty}$-module $E_{0} \otimes_{\mathbf{z}_{p}} \mathbf{F}_{p}$ by $\left(E_{0}\right)_{\mathbf{F}_{p}}$. Note that since over $T^{\text {log }}$, the obstruction to putting a full connection on $\mathscr{E}_{F_{b}}$ vanishes, so we get a genuine $\mathcal{M F}^{\nabla_{-}}$ object (whose underlying vector bundle is $\left.\left(\mathscr{E} \mathscr{D} \otimes \mathscr{D}_{S} / \mathscr{I}_{S}\right)_{\mathbf{F}_{p}}\right)$ modulo $p$. Thus, the $\Pi_{\infty}$-action on $\left(E_{0}\right) \mathbf{F}_{p}$ extends to a natural (dual crystalline) action of $\Pi_{1}$ on $\left(E_{0}\right)_{\mathbf{F}_{p}}$. We apply Schlessinger's theory to the functor on $\operatorname{artinian}$ rings $B$ with residue field $\mathbb{F}_{p}$ that assigns to such a ring $B$ the set of isomorphism classes of continuous representations of $\Delta$ on a free $B$-module $E_{B}$ of rank two such that $\left(E_{B}\right) \otimes_{B} \mathbb{F}_{p}=\left(E_{0}\right)_{F_{p}}$. Since $H^{1}\left(\Delta . \operatorname{Ad}\left(\left(E_{0}\right)_{F_{p}}\right)\right)$ is zero if $i \neq 1$, and of dimension $6 g-6+2 r$ over $\mathbb{F}_{p}$ if $i=1$, it follows easily from [Schl] that this functor is prorepresented by a formal scheme $\mathscr{R}$ over $\mathbb{Z}_{p}$. Moreover, $\mathscr{R}$ is formally smooth over $\mathbb{Z}_{p}$, of relative dimension $6 g-6+2 r$.

Now we claim that there is a natural continuous action of $\pi_{1}\left(\left(T^{\log }\right)_{K}\right)$ on $\mathscr{R}$. Indeed, let $\alpha \in \Pi_{1}$. Since $\Delta \subseteq \Pi_{1}$ is a normal subgroup, for any representation of $\Delta$ on some $E_{B}$ as above, we obtain a new representation by conjugating elements of $\Delta$ by $\alpha$, and then acting on $E_{B}$ in the original fashion. Since the original $\Delta$-action on $E_{B}$ extends to a full $\Pi_{1}$-action on $\left(E_{B}\right) \otimes_{B} \mathbb{F}_{p}$, it follows that this new representation is isomorphic to the old after base change by $B \rightarrow \mathbb{F}_{p}$. Thus, the new representation defines a new $B$-valued point of $\mathscr{R}$. This defines an action of $\Pi_{1}$ on $\mathscr{R}$ which is clearly trivial on $\Delta \subseteq \Pi_{1}$. Thus, we obtain a natural action of $\pi_{1}\left(\left(T^{\log }\right)_{K}\right)$ on $\mathscr{R}$.

Let us now turn to applying $\mathscr{R}$ to understanding the $\Pi_{1}$-module $E_{X}^{\mathrm{Ga}}$. Let $\widehat{\mathscr{D}}_{S}^{\mathrm{Gal}}$ be the $p$-adic completion of $\mathscr{D}_{S}^{\mathrm{Gal}}$. The underlying $\Delta$-module structure on $E_{X}^{\mathrm{Gal}}$ defines a classifying morphism

$$
\kappa: \operatorname{Spf}\left(\widehat{\mathscr{D}}_{\mathrm{G}}^{\mathrm{Gal}}\right) \rightarrow \mathscr{R}
$$

(This is O.K. despite the fact that $\widehat{\mathscr{D}}_{S}^{\text {Gal }} \otimes_{Z_{p}} \mathbb{Z} / p^{N} \mathbb{Z}$ is not artinian, since $\Delta$ is topologically finitely generated.) Let

$$
\sigma_{0}: \operatorname{Spf}\left(\mathbb{Z}_{p}\right) \hookrightarrow \mathscr{R}
$$

be the composite of $\operatorname{Spf}\left(\pi_{S}\right)$ with $\kappa$. Let $\mathscr{R}^{\mathrm{PD}}$ be the $p$-adic completion of the PD-envelope of $\mathscr{R}$ at $\sigma_{0}$. Let

$$
\kappa^{\mathrm{PD}}: \operatorname{Spf}\left(\widehat{\mathscr{D}}_{S}^{\mathrm{Gal}}\right) \rightarrow \mathscr{R}^{\mathrm{PD}}
$$

be the morphism induced by $\kappa$. Then the morphism induced by $\kappa^{\mathrm{PD}}$ on the Zariski tangent spaces at $\sigma_{0}$ is precisely the injection corresponding to the class $\eta^{\text {Gal }}$ considered above. Thus, we have the following: 
Lemma 1.5. The morphism $\kappa^{\mathrm{PD}}$ is a closed immersion of formal schemes.

Now let us consider the fact that the $\Delta$-module structure on $E_{X}^{\mathrm{Gal}}$ actually comes from a $\Pi_{1}$-module structure which is $\mathscr{D}_{S}^{\text {Gal }}$-semilinear (with respect to the $\Pi_{1}$-action on $\left.\mathscr{D}_{S}^{\mathrm{Gal}}\right)$-though $\pi_{1}\left(T_{K}^{\text {log }}\right)$ ). If we translate this statement by means of the functorial interpretation of $\mathscr{R}$, we obtain the following:

Lemma 1.6. The morphism $\kappa$ is $\pi_{1}\left(T_{K}^{\mathrm{log}}\right)$-equivariant with respect to the natural $\pi_{1}\left(T_{K}^{\mathrm{log}}\right)$-actions on $\widehat{\mathscr{D}}_{\mathrm{S}}^{\mathrm{Gal}}$ and $\mathscr{R}$.

Now let $\phi: \Gamma \rightarrow \pi_{1}\left(T_{K}^{\log }\right)$ be a continuous homomorphism of topological groups. Let

$$
1 \rightarrow \Delta \rightarrow \Pi_{\Gamma} \rightarrow \Gamma \rightarrow 1
$$

be the pull-back of the group extension

$$
1 \rightarrow \Delta \rightarrow \mathbb{\Pi}_{1} \rightarrow \pi_{1}\left(T_{K}^{\text {log }}\right) \rightarrow 1
$$

by means of $\phi$. Then one can consider the issue of whether or not the $\Delta$-action on $E_{0}$ extends to a continuous, $\mathbb{Z}_{p}$-linear action of $\mathbb{\Pi}_{\Gamma}$ on $E_{0}$. Note that since $H^{0}\left(\Delta, \operatorname{Ad}\left(E_{0}\right)\right)=0$, as long as we require that the associated determinant representation of $\Pi_{\Gamma}$ is the cyclotomic character, such an extension will always be unique (up to \pm 1 ). On the other hand, by the same reasoning as that used in Lemma 1.6 , the $\Delta$-action on $E_{0}$ will extend to a $\Pi_{\Gamma}$-action on $E_{0}$ if and only if the $\mathbb{Z}_{p}$-valued point $\sigma_{0}$ of $\mathscr{R}$ is fixed by $\Gamma$ (acting through $\phi$ ). Moreover, by the preceding two lemmas, we see that $\sigma_{0}$ is fixed by $\Gamma$ if and only if the $\mathbb{Z}_{p}$-valued point of $\widehat{\mathscr{D}}_{S}^{\text {Gal }}$ defined by $\pi_{S}$ is stabilized by $\Gamma$. But this, in turn, is equivalent to the statement that the restriction of the canonical extension class $\eta_{\Phi}$ (discussed just before Definition 1.5 of Chapter III) becomes trivial when restricted to $\Gamma$.

Now let us suppose that $\left(B, \mathfrak{m}_{B}\right)$ is a local ring with residue field $k$ which is $p$-adically complete, $\mathbb{Z}_{p}$-flat, and has a topologically nilpotent PD-structure on $\mathfrak{m}_{B}$. (Note that for such a ring $B, \log :\left(1+\mathfrak{m}_{B}\right) \rightarrow \mathfrak{m}_{B}$ and exp: $\mathfrak{m}_{B} \rightarrow$ $\left(1+m_{B}\right)$ define inverse isomorphisms.) Let $\Gamma^{\prime}=\pi_{1}\left(\operatorname{Spf}(B)_{K}\right)$. Suppose further that

$$
\phi: \operatorname{Spf}(B) \rightarrow S
$$

is a morphism whose image avoids the divisor defining the log structure on $S^{\log }$. Then for some closed subgroud $\Gamma \subseteq \Gamma^{\prime}$ of finite index, we have a morphism $\phi$ : $\Gamma \rightarrow \pi_{1}\left(T_{K}^{\log }\right)$ which is compatible with the morphism induced on fundamental 
groups by $\phi$. Recall from Chapter III, Theorem 3.8, the canonical affine coordinates corresponding to $\phi$. These coordinates are valued in $B$ (or, more precisely, in $\mathfrak{m}_{B}$ ). Moreover, they are zero if and only if the class $\eta_{\Phi}$ becomes zero when restricted to $\Gamma^{\prime}$. It is easy to see that $\left.\eta_{\Phi}\right|_{\Gamma^{\prime}}=0$ if and only if $\left.\eta_{\Phi}\right|_{\Gamma}=$ 0 . Also, we know from Chapter III that $\phi$ corresponds to a canonical curve if and only if the canonical affine coordinates are zero. Thus, putting everything together, we obtain the following result:

Theorem 1.7. The morphism $\phi$ corresponds to a canonical curve if and only if the $\Delta$-action on $E_{0}$ extends to a $\mathbb{Z}_{p}$-linear, continuous action of $\Pi_{\Gamma}$ on $E_{0}$.

More generally, but less precisely, we see that:

(*) The canonical affine coordinates in $\mathrm{m}_{B}$ associated to $\phi$ are a measure of the obstruction to extending the $\Delta$-action on $E_{0}$ to a $\mathbb{Z}_{p}$-linear, continuous action of $\Pi_{\Gamma}$ on $E_{0}$.

Since the class $\eta_{\Phi}$ is "as nonzero as it can be" on $S^{\text {log }}$, we thus see that we have justified the statement made at the end of the preceding subsection that the $\Pi_{1}$-module $E_{X}^{\mathrm{Gal}}$ is "as close as one can get" to extending the $\Delta$-action on $E_{0}$ to a full $\Pi_{1}$-action.

Remark. In some sense, we can describe what we have done in this subsection as follows. Consider the obstruction to extending the $\Delta$-action on $E_{0}$ to an action of $\Pi_{\Gamma}$. A priori, this obstruction is highly nonlinear and difficult to get one's hands on explicitly. Note that this nonlinearity exists despite the fact that we already have a $\Pi_{\infty}$-action on $E_{0}$, and the discrepancy between $\Pi_{1}$ and $\Pi_{\infty}$ is "essentially" a linear $\mathbb{Z}_{p}$-space of rank $3 g-3+r$. Rather, the nonlinearity arises fundamentally from the fact that we are considering representations into $\mathrm{GL}_{2}^{ \pm}$, which is not an abelian (or even solvable) group. In particular, the moduli space $\mathscr{R}$ of representations of $\Delta$ into $G_{2}^{ \pm}$has no natural linear structure. Thus, the point of constructing $E_{X}^{\mathrm{Gal}}$ and reinterpreting the existence of $E_{X}^{\mathrm{G} a l}$ in terms of $\mathscr{R}$, as we have done in this subsection, was to linearize this obstruction by means of the uniformization of (the relevant part of) $\mathscr{R}^{\mathrm{PD}}$ by means $\kappa^{\mathrm{PD}}$.

Remark. For the reader interested in pursuing analogies with the complex case, we also make the following observation. Since $\mathscr{R}$ is the local moduli space of deformations of the canonical representation $\Delta \rightarrow \mathrm{GL}_{2}^{ \pm}\left(\mathbf{Z}_{p}\right)$ of the geometric fundamental group, it is natural to regard $\mathscr{R}$ as a sort of local $p$-adic analogue of the space $\Re_{\mathrm{C}}$ of isomorphism classes of representations of the geometric fundamental group into $\mathrm{PSL}_{2}(\mathbf{C})$ in the complex case. Thus, $\mathscr{R}_{\mathbf{C}}$ has complex dimension $6 g-6+2 r$. Inside $\mathscr{R}_{\mathbf{C}}$, one has Fricke space $\mathscr{R}_{\mathbf{R}}$, with real dimension $6 r-6+2 r$, corresponding to the representations into $\mathrm{PSL}_{2}(\mathbf{R})$. In a neighbor- 
hood of the canonical representation of a curve, $\mathscr{R}_{\mathbb{R}}$ maps diffeomorphically onto $M_{g, r}$ Thus, one can regard the subspace $\operatorname{Spf}\left(\widehat{\mathscr{D}}_{S}^{\mathrm{Gal}}\right) \hookrightarrow \mathscr{R}^{\mathrm{PD}}$ as analogous to Fricke space $\mathscr{R}_{\mathbb{R}} \subseteq \mathscr{R}_{\mathbf{C}}$ in the complex case.

\section{The Parabolic Case}

Before proceeding, we pause to take a brief look at what happens for elliptic curves (regarded parabolically). Although there are many similarities between the parabolic and hyperbolic cases, there are also certain differences. This is not so surprising if one considers the canonical representations arising from uniformizations in the complex case. Indeed, in the complex context, for hyperbolic curves, the canonical representation of the fundamental group into $\mathrm{PSL}_{2}(\mathbb{R})$ is completely well-defined up to conjugation by an element of $\mathrm{PSL}_{2}(\mathbb{R})$, while for elliptic curves, the morphism (induced by deck transformations on the uniformization by $\mathbb{C}$ ) gives a representation of the fundamental group into the group of translations $\mathbb{G}_{a}(\mathbb{C})$ of the complex plane, but this representation is not well-defined up to conjugation by an element of $\mathbb{G}_{a}(\mathbb{C})$. Rather, there is an ambiguity of multiplication by a complex number.

On the other hand, this same phenomenon of "lack of rigidity" ultimately is a consequence of the overall linearity of the situation, which has positive aspects, as well. For instance, one can carry out the construction of the induced $M F^{\nabla}$-object (Theorem 1.1) for elliptic curves just as in the hyperbolic case. However, precisely because the obstruction to defining an $M \mathscr{F}^{-} \nabla_{\text {-object }}$ is entirely linear from the outset, this approach is a sort of overkill. Thus, in the following we propose to examine the obstruction to defining a "full" $M \mathscr{F}^{\nabla}$-object (i.e., the same obstruction as the one we examined in the hyperbolic case) directly, at the level of Galois representations, without resorting to the tool of crystalline induction.

Thus, let us assume that $f^{\log }: X^{\log } \rightarrow S^{\log }$ is an ordinary zero pointed curve of genus one such that the classifying morphism $S \rightarrow \bar{M}_{1,0}$ is étale. Since the canonical representation should be an extension of a rank one étale representation by its dual Tate twisted once, we consider the local system $\mathbb{R}^{1}\left(f_{K}^{10 g}\right)_{\mathrm{et}, * \mathbb{Z}_{p}(1)}$ on $S_{K}^{\mathrm{log}}$. Let us denote the $\prod_{S} \stackrel{\text { def }}{=} \pi_{1}\left(S_{K}^{\mathrm{log}}\right)$-module corresponding to this local system by $H^{1}$. Then there exists a rank one $\Pi_{S^{-}}$submodule $E(1) \subseteq H^{1}$ such that the action of $\Pi_{S}$ on $E$ is unramified, and we have an exact sequence:

$$
0 \rightarrow E(1) \rightarrow H^{1} \rightarrow E^{\vee} \rightarrow 0
$$

Suppose that $E^{\prime}$ is an étale $\Pi_{S}$-module such that $\left(E^{\prime}\right)^{\otimes 2}=E^{\vee}$. Then, the canonical representation of the universal elliptic curve should be an extension of $E^{\prime}$ by $\left(E^{\prime}\right)^{\vee}(1)$. If we tensor the above exact sequence by $E$, we get an exact 
sequence of $\Pi_{S}$-modules

$$
0 \rightarrow E^{\otimes 2}(1) \rightarrow H^{1} \otimes E \rightarrow \mathbf{Z}_{p} \rightarrow 0
$$

Thus, the obstruction to the existence of such a canonical representation is precisely the obstruction to lifting $1 \in \mathbb{Z}_{p}$ in the above exact sequence. This obstruction class lies in

$$
H^{1}\left(\Pi_{S}, E^{\otimes 2}(1)\right)
$$

and coincides with the class $\eta_{\Phi}$ defined by the canonical Frobenius lifting $\Phi^{100}$ on $S^{\log }$. That is to say, we end up with essentially the same conclusion as in the hyperbolic case: Namely, that the obstruction to the existence of a "canonical representation" for the universal ordinary elliptic curve (defined on all of $\left.\pi_{1}\left(X_{K}^{\text {log }}\right)\right)$ is given precisely by the class $\eta_{\Phi}$ defined by the canonical Frobenius lifting.

\section{§2. Canonical Objects Over the Stack of Multiplicative Periods}

In this Section, we note that by working over the stack of multiplicative periods, we can construct all the objects that we are familiar with from the case of canonical curves.

\section{The Stack of Multiplicative Periods}

Let $S^{\log }=\left(\overline{\mathcal{N}}_{g, r}^{\text {ord }}\right)^{\log }$. Let $\Phi^{\log }$ be the canonical Frobenius lifting on $S^{\log }$ (as in Chapter III, Theorem 2.8). If, for some $N \geq 1$, we take the $N^{\text {th }}$ iterate of $\Phi^{\log }$. we get a finite, flat covering

$$
\left(\Phi^{\log }\right)^{N}: S^{\log } \rightarrow S^{\log }
$$

of $S^{\log }$. Let $P^{\text {log }}$ be the inductive limit of these coverings (as $N$ goes to infinity). Let $\widehat{P}^{\text {log }}$ be the $p$-adic completion of $P^{\text {log }}$.

Definition 2.1. We shall call $P^{\text {log }}$ (respectively, $\widehat{P}^{\mathrm{log}}$ ) the universal (respectively, complete) stack of multiplicative periods.

Unlike the rings of additive periods considered earlier, which, roughly speaking, are generated by adjoining the logarithms of the multiplicative parameters to $\mathbf{Z}_{p}$, the structure sheaf of the stack of multiplicative periods is obtained essentially 
by adjoining all $p$-power roots of the multiplicative parameters to the structure sheaf of the original base scheme (or stack).

More generally, let $T^{\log }$ be a formal log scheme, whose underlying scheme $T$ is $p$-adically complete and flat over $\mathbb{Z}_{p}$. Let $h^{\log }: Y^{\log } \rightarrow T^{\log }$ be an $r$-pointed stable curve of genus $g$ that arises from some classifying morphism $T^{\log } \rightarrow S^{\log }$. Then if we pull back the morphism $P^{\log } \rightarrow S^{\log }$ via the classifying morphism $T^{\log } \rightarrow S^{\log }$ for $h^{\log }$, we obtain an object

$$
P_{T}^{\log } \rightarrow T^{\log }
$$

over $T^{\text {log }}$. Let $\widehat{P}_{T}^{\text {log }}$ be the $p$-adic completion of $P_{T}^{\text {log }}$. We shall always assume that:

$\left.{ }^{*}\right) \quad$ The log structure of $T_{\mathbf{Q}_{p}}^{\log }$ is trivial over an open dense set.

For instance, typically $T^{\log }$ will be the normalization of $\mathbb{Z}_{p}$ in a finite field extension of $\mathbb{Q}_{p}$ with a log structure that is trivial in characteristic zero.

Befinition 2.2. We shall call $P_{T}^{\text {log }}$ (respectively, $\widehat{P}_{T}^{\text {log }}$ ) the (respectively, completed) formal scheme of multiplicative periods associated to the curve $h^{\log }: Y^{\text {tog }}$ $\rightarrow T^{\log }$.

Note that $P_{T}^{\log }$ depends on the choice of classifying morphism $T^{\log } \rightarrow S^{\log }$ for the curve that lifts the morphism $T^{\log } \rightarrow \bar{M}_{g, r}^{\log }$ defined by the curve itself. That is, $P_{T}^{\log }$ depends on a choice of quasiconformal equivalence class for $h^{\log }: Y^{\log } \rightarrow T^{\log }$.

Remark. Often in what follows we shall work in the universal case, that is, over $S^{\log }$, and thus obtain objects over $P^{\log }$ (or $\widehat{P}^{\mathrm{log}}$ ). However, one should always remember that the objects constructed define (by restriction) objects over $P_{T}^{\text {log }}$ (or $\widehat{P}_{T}^{\text {log }}$ ) for any $r$-pointed stable curve of genus $g$ over $T^{\log }$ satisfying the hypotheses just stated.

\section{The Canonical $\mathbb{L} \circ g$ p}

Let $S^{\log }=\left(\mathcal{N}_{g, r}^{\text {ord }}\right)^{\text {log: }}$ that is, the locus of smooth ordinary curves (with a choice of quasiconformal equivalence class). Thus, the $\log$ structure on $S^{\log }$ is trivial. Let $f^{\log }: X^{\log } \rightarrow S^{\log }$ be the universal curve. Let $f^{\log }[N]: X^{\log }[N] \rightarrow$ $S^{\log }$ be the pull-back of $f^{\log }$ by the $N^{\text {th }}$ iterate of the canonical Frobenius. Thus, if we pull $\left(\mathscr{E}, \nabla_{\mathscr{E}}\right)$ back to $X^{\log }[N]$ and reduce modulo $p^{N}$, the obstruction to defining a full connection (relative to $X^{\log }[N] \rightarrow \operatorname{Spec}(A)$ ) vanishes, and so, we 
obtain an $\mathcal{M F}^{\nabla_{-}}$object, which we shall call $\mathscr{E}[N]$. Alternatively, this $\mathcal{M F}^{\nabla_{-}}$ object can be obtained by taking the $\mathcal{M} \mathscr{F}^{\nabla}$-object in Theorem 1.1, pulling back to $X^{\mathrm{log}}[N]$, reducing modulo $p^{N}$, and then reducing modulo $\mathscr{I}_{s}$. By [Falt], Theorem 7.1, away from the divisor at infinity, and the method of Chapter IV, $\S 2$ (following [Kato 2]) at the divisor at infinity, $\mathscr{E}[N]$ defines a finite, flat log group object $G[N]$ on $X^{\log }[N]$. Let $P^{\text {log }} \rightarrow S^{\log }$ be the stack of multiplicative periods. Let $f^{\log }[\infty]: X^{\log }[\infty] \rightarrow P^{\log }$ be the pull-back of $f^{\log }$ to $P^{\log }$. Then by restricting from $X^{\log }[N]$ to $X^{\log }[\infty]$ and then taking the inductive limit, we obtain an (up to \pm 1 ) $\log p$-divisible group

$$
G[\infty]=\left.\underset{\longrightarrow}{\lim } G[N]\right|_{X^{\log }[\infty]}
$$

on $X^{\log }[\infty]$.

Definition 2.3. We shall call $G[\infty]$ the canonical $\log p$-divisible group on $X^{\log }[\infty]$.

If we invert $p$, and pass to $p$-adic Tate modules, then we obtain an étale local system of $\mathbb{Z}_{p} \oplus \mathbf{Z}_{p}$ 's (up to \pm 1 ) on $X^{\log }[\infty]_{\mathbf{Q}_{p}}$. Now in the notation of $\S 1$, we have

$$
\Pi_{\infty}=\pi_{1}\left(X^{\log }[\infty]_{Q_{\rho}}\right)
$$

Thus, the $p$-adic Tate module of $G[\infty]_{\mathbf{Q}_{0}}$ is given by the representation of $\Pi_{\infty}$ on $E_{0}$ which was discussed in $\S 1$. Let us denote this representation by

$$
\rho_{\infty}: \Pi_{\infty} \rightarrow \mathrm{GL}^{ \pm}\left(E_{0}\right)
$$

Definition 2.4. We shall call $\rho_{\infty}$ the canonical Galois representation of $\Pi_{\infty}$.

Remark. If $T$ is any $p$-adically complete formal scheme which is $\mathbf{Z}_{p}$-flat, and $\phi: T \rightarrow \mathcal{N}_{g, r}^{\text {ord }}$ is a morphism, then even though the canonical log $p$-divisible group and the canonical Galois representation are not defined until one goes up to the scheme of multiplicative periods, one can nonetheless pull-back the canonical indigenous bundle $\left(\mathscr{E}, \nabla_{\mathscr{E}}\right)_{\mathcal{N}}$ on $\mathcal{N}_{\boldsymbol{g}, r}^{\text {ord }}$ to obtain an indigenous bundle $\left(\mathscr{E}, \nabla_{\mathscr{E}}\right)_{T}$ on $X_{T}^{\log } \rightarrow T$. This indigenous bundle is defined over $T$, i.e., one needn't pass to the scheme of multiplicative periods.

Definition 2.5. We shall call $\left(\mathscr{E}, \nabla_{\mathscr{E}}\right)_{T}$ the canonical indigenous bundle on $X_{T}^{\mathrm{log}}$. 
One should always remember that one only obtains the canonical indigenous bundle after choosing a lifting $\phi: T \rightarrow \mathcal{N}_{g, r}^{\text {ord }}$ (i.e., a quasiconformal equivalence class) of the classifying morphism $T \rightarrow M_{g, r}$ of the curve.

\section{The Canonical Frobenius Lifting}

We continue with the notation of the preceding subsection. It follows from Chapter IV, Proposition 3.2, that the supersingular divisor $D \subseteq X_{\mathrm{F}_{0}}$ (where $f^{\mathrm{og}}$ : $X^{\log } \rightarrow S^{\log }$ is the universal curve) is étale over $S_{F_{p}}$. We shall denote its complement in $X$, the ordinary locus of $X^{\mathrm{log}}$, by $X^{\text {ord }}$. Now it is immediate that the construction of the canonical Frobenius lifting over the ordinary locus (preceding Theorem 1.6 of Chapter IV) carries over immediately to the present case (where the base is $S$, as opposed to the ring of Witt vectors with coefficients in a perfect field). Thus, if we denote base-change by $\Phi^{\log }$ (the canonical Frobenius) by means of a superscript " $F$," we obtain the universal analogue of Theorem 1.6 of Chapter IV:

Theorem 2.6. There exists a unique ordinary Frobenius lifting (called canonical)

$$
\Phi_{X}^{\log }:\left(X^{\mathrm{log}}\right)^{\text {ord }} \rightarrow\left(\left(X^{\mathrm{log}}\right)^{\text {ord }}\right)^{F}
$$

over the ordinary locus such that we get a horizontal morphism $\Phi_{X}^{*} \mathscr{E} F \rightarrow \mathscr{E}$ which preserves the Hodge filtration.

Now let $T$ be any $p$-adically complete formal scheme which is $\mathbb{Z}_{p}$-flat, and let $\phi: T \rightarrow \mathcal{N}_{g, r}^{\text {ord }}$ be a morphism. Let $X_{T}^{\text {log }} \rightarrow T$ be the pull-back of the universal curve by $\phi$. Write $X_{T}^{\log }$ for the pull-back of the universal curve by $\Phi \circ \phi$. Then by restricting the morphism $\Phi_{X}^{\text {log }}$ of Theorem 2.7, we obtain a $T$-morphism

$$
\Phi_{X_{T}}^{\log }:\left(X^{\log }\right)_{T}^{\underset{T}{\text { ord }}} \rightarrow\left(X^{\log }\right)_{T^{F}}^{\text {ord }}
$$

Thus, in the spirit of Definition 4.18 of Chapter IV, we make the following:

Definition 2.7. We shall call $X_{T^{F}}^{\mathrm{log}}$ the Frobenius conjugate curve to $X_{T}^{\mathrm{log}}$. We shall call $\Phi_{X_{T}^{\prime o g}}^{\log }$ the $p$-adic Green's function of the curve $X_{T}^{\mathrm{log}} \rightarrow T$.

Next, we consider compactifications of this canonical Frobenius lifting. Let $D[\infty] \subseteq X[\infty]_{\mathbf{F}_{p}}$ be the result of base-changing to $\widehat{P}$. Let $D[\infty] \subseteq \widehat{X}[\infty]$ be the respective $p$-adic completions. Let $\widehat{X}_{D}[\infty]$ be the completion of $\widehat{X}[\infty]$ at 
$D[\infty]$. Then just as in $\S 3$ of Chapter IV, by looking at the universal deforma. tion spaces of the canonical $\log p$-divisible group and its double Frobenius conjugate, we obtain an isomorphism

$$
\Psi: \widehat{X}_{D^{p 2}}[\infty] \cong \widehat{X}_{D}[\infty]
$$

Let $\widehat{Y}[\infty] \rightarrow \widehat{X}[\infty]$ be the finite, flat covering (of degree $p+1$ ) parametrizing cyclic subgroups (in the Drinfeldian sense) of the canonical log $p$-divisible group. Then, just as before, there exists a divisor $E \subseteq \widehat{Y}[\infty]_{\mathbf{F}_{p}}$ that maps isomorphically onto $D[\infty]_{\mathrm{F}_{p}}$. Let $\widehat{Y}_{E}[\infty]$ be the completion of $\widehat{Y}[\infty]$ at $E$. Then we obtain an embedding

$$
\left(\mathscr{L}_{E}, \mathscr{R}_{E}\right): \widehat{Y}_{E}[\infty] \rightarrow \widehat{X}_{D}[\infty] \times \widehat{P}_{\widehat{X}} \widehat{X}_{D^{f^{2}}}[\infty]
$$

The image of this embedding is a divisor, which, when restricted to the ordinary locus, is equal to the union of the graph of the canonical Frobenius on ( $X^{\mathrm{log}}$ ) ord with its " $\Psi$-transpose," as in Chapter IV, §3 (see the discussion preceding Definition 3.3).

Definition 2.8. We shall call $\widehat{Y}[\infty] \rightarrow \widehat{X}[\infty]$, together with $\Psi$ and $\left(\mathscr{L}_{E}\right.$, $\mathscr{R}_{E}$ ) the compactification of the canonical Frobenius lifting $\Phi_{X}^{\text {log }}$.

Thus, although the canonical Frobenius lifting $\Phi_{X}^{\text {log }}$ is defined over $S$ (without passing to the stack of multiplicative periods), the compactification is only defined over the completed stack of multiplicative periods.

\section{Bibliography}

[Abikoff] Abikoff, W.. The Real Analytic Theory of Teichmuller Space, Lecture Notes in Math., 820, Springer, 1980.

[AC] Arbarello, E. and Cornalba, M., The Picard Groups of the Moduli Space of Curves, Topology, 26, No. 2, (1987), 153-171.

[BBM] Berthelot, P., Breen, L. and Messing, W.. Théorie de Dieudonné cristalline II, Lecture Notes in Math., 930, Springer, 1982.

[Bers] Bers, L., Uniformization by Beltrami Equations, Comm. Pure Appl. Math., 14. (1961), 215-228.

[BK] Bloch, S. and Kato, K., L-Functions and Tamagawa Numbers in The Grothendieck Festschrift I, Birkhauser (1990), 333-400.

[EGA IV] Grothendieck A., and Dieudonné, J., Eléments de Géometrie Algébrique IV. 
Publ. Math. IHES, 32, (1967).

[Falt] Faltings, G., Crystalline Cohomology and $p$-adic Galois Representations, JAMI Conference, Johns Hopkins Univ. Press, (1990), 25-79.

[Falt2] - Real Projective Structures on Riemann Surfaces, Compositio Math., 48, Fasc. 2, (1983), 223-269.

[Falt3] - Stable G-Bundles and Projective Connections, J. Algebraic Geom., 2, No. 3, (1993), 507-568.

[FK] Farkas, H. and Kra, I., Riemann Surfaces, Graduate Texts in Math. 71, Springer, 1980.

[Gard] Gardiner, F., Teichmüller Theory and Quadratic Differentials, John Wiley and Sons, 1987.

[Gunning] Gunning, R. C., Special Coordinate Covering of Riemann Surfaces, Math. Ann., 170, (1967), 67-86.

[Harts] Hartshorne, R., Residues and Duality, Lecture Notes in Math., 20. Springer, 1966.

[HM] Harris, J. and Mumford. D., On the Kodaira Dimension of the Moduli Space of Curves, Inv. Math., 67, (1982), 23-86.

[Ih] Ihara, Y., Schwarzian Equations, J. Fac. Sci. Univ. Tokyo, Sect IA Math., 21, (1974), 97-118.

[Ih2] - On the Differentials Associated to Congruence Relations and the Schwarzian Equations Defining Uniformizations, J. Fac. Sci. Univ. Tokyo, Sect. IA Math., 21, (1974), 309-332.

[Ih3] - On the Frobenius Correspondences of Algebraic Curves, in Algebraic Number Theory, papers contributed for the International Symposium, Kyoto, 1976, Japan Snc. Prom. Sci., Tokyo. 1977

[Ih4] - L Lifting Curves over Finite Fields Together with the Characteristic Correspondence II+II', J. Algebra, 75, No. 2, (1982). 452-483.

[Jac] Jacobson, N., Lie Algebras, Interscience Tracts 10, John Wiley and Sons, 1962.

[Kato] Kato, K., Logarithmic Structures of Fontaine-Illusie. Proceedings of the First JAMI Conference, Johns-Hopkins University Press (1990), 191-224.

[Kato2] - Logarithmic Degeneration and Dieudonne Theory, Preprint.

[Katz] Katz, N., Nilpotent Connections and the Monodromy Theorem: Applications of a Result of Turritin, Publ. Math. IHES, 39, (1970), 355-412.

[Katz2] - Algebraic Solutions of Differential Equations, Inv. Math., 18, (1972), $1-118$.

[KM] Katz, N. and Mazur, B., Arithmetic Moduli of Elliptic Curves, Ann. of Math. Stud., 108, Princeton Univ. Press. 1985.

[Lang] Lang, S., Introduction to Modular Forms, Grundlehren Math. Wiss. 222, Springer, 1976.

[Lehto] Lehto, O., Univalent Functions and Teichmüller Spaces, Graduate Texts in Math. 109, Springer, 1987.

[Lehto2] - Quasiconformal Homeomorphisms and Beltrami Equations, in Discrete Groups and Automorphic Functions, ed. W. J. Harvey, Academic Press, (1977), 121-142. 
[Marg] Margulis, G. A., Discrete Subgroups of Semisimple Lie Groups, Ergeb. Math. Grenzgeb. 17, Springer, 1990.

[McM] McMullen, C., Rational Maps and Kleinian Groups, Proc. International Congress of Mathematicians II, Kyoto, (1990), 889-899.

[Mess] Messing, W., The Crystals Associated to Barsotti-Tate Groups; with Applications to Abelian Schemes, Lecture Notes in Math., 264, Springer, 1972.

[Mzk] Mochizuki, S., The Geometry of the Compactification of the Hurwitz Scheme, Publ. RIMS. Kyoto Univ., 31. No. 3, (1995), 355-441.

[Ogus] Ogus, A., F-Crystals, Griffiths Transversality, and the Hodge Decomposition, Astérisque, 221, (1994).

[Royd] Royden, H. L., Intrinsic Metrics on Teichmüller Space, Proc. International Congress of Mathematicians 2. Vancouver. (1974), 217-221.

[Schl] Schlessinger, M., Functors of Artin Rings, Trans. Amer. Math. Soc., 130, (1968), 208-222.

[Sesh] Seshadri, C. S., Fibrés vectoriels sur les courbes algébriques, Astérisque, 96, (1982).

[SGA1] Grothendieck, A. et al., Revêtements êtales et groupe fondamental, Lecture Notes in Math. 224. Springer, 1971.

[Shi] Shimura, G., Introduction to the Arithmetic Theory of Automorphic Forms, Publ. Math. Soc. Japan 11. Iwanami Shoten and Princeton Univ. Press, 1971.

[Tate] Tate, J., p-divisible Groups, Proceedings of a Conference on Local Fields, NUFFIC Summer School held at Driebergen in 1966, Springer, 1967.

[Wolp] Wolpert, S., The Fenchel-Nielsen Deformation, Ann. Math., 115, (1982). 501-528.

[ZT] Zograf, P. G. and Takhtadzhyan, L. A., On Uniformization of Riemann Surfaces and the Weil-Petersson Metric on Teichmüller and Schottky Spaces, Math. USSR Sbomik, 60, No. 2, (1988), 297-313.

\section{Index}

\section{Terminology}

Note: The data following each term indicate the chapter and section where the term first appears.

admissible compactified Frobenius lifting IV .4

admissible Frobenius lifting IV.4

admissible indigenous bundle II.2

associated Schwarz structure $\quad$ I.1

$\begin{array}{ll}\text { Beltrami differential Intro.1 } & \text { Int }\end{array}$ 


\begin{tabular}{|c|c|}
\hline Beltrami equation & Intro.1 \\
\hline Bers embedding & Intro.1 \\
\hline bianalytic constant & I.1 \\
\hline function & I.1 \\
\hline uniformizer & I.1 \\
\hline biformal function & I.1 \\
\hline canonical affine parameter & III. 1 \\
\hline canonical bilinear form & II.3, III.3 \\
\hline canonical class & III. 3 \\
\hline canonical (crystalline) Galois representation & IV.1, V.2 \\
\hline canonical curve & III. 3 \\
\hline canonical Frobenius lifting (modular) & III. 2 \\
\hline canonical Frobenius lifting (on a curve) & IV.1, V.2 \\
\hline canonical Galois extension class & III. 1 \\
\hline canonical height & $\mathrm{I} .2$ \\
\hline $\begin{array}{l}\text { canonical indigenous bundle } \\
\text { canonical lifting (point) }\end{array}$ & $\begin{array}{l}\text { Intro.2, V.2 } \\
\text { III.1 }\end{array}$ \\
\hline canonical local Hecke correspondence, $n^{\text {th }}$ & IV.3 \\
\hline canonical local systems & III.1, III.3 \\
\hline canonical $\log p$-divisible group & IV.2, V.2 \\
\hline canonical multiplicative parameter & III. 1, III. 3 \\
\hline canonical $p$-divisible group & III. 1 \\
\hline canonical pseudo-Hecke correspondence, $n^{t h}$ & IV .3 \\
\hline canonical representation (complex case) & Intro.1, Intro.2 \\
\hline canonical uniformization & III. 3 \\
\hline compactification of a Frobenius lifting & IV .4 \\
\hline compactification of the canonical Frobenius lifting & V.2 \\
\hline crystalline-induced Galois representation & V.1 \\
\hline crystalline induction & V.0 \\
\hline crystalline Schwarz structure & I.1 \\
\hline with nilpotent monodromy & I.1 \\
\hline differential local system & III.1, III.3 \\
\hline dilatation & Intro. 1 \\
\hline divisor of marked points & I. 1 \\
\hline elliptic Riemann surface & Intro. 1 \\
\hline Fenchel-Nielsen coordinates & Intro. 1 \\
\hline finite type (Riemann surface of) & Intro. 1 \\
\hline$F L$-bundle & II .1 \\
\hline
\end{tabular}


Frobenius conjugate curve

V.2

Frobenius lifting

III.1

Frobenius on $\mathbf{R}^{1} f_{*} \tau_{\chi^{108} / S^{\text {Iug }}}$

II. 2

generic uniformization number

II. 3

global $n^{\text {th }}$ canonical Hecke correspondence

IV .3

Green's function (complex case)

Intro. 1

Green's function, $p$-adic

IV . 4, V.2

Hasse invariant

II .2 , II. 3

Hecke operators

IV .3

Hecke type

IV .3

height of a Frobenius lifting

IV .4

Hilbert transformation

Intro. 1

Hodge section

I. 2

hyperbolic metric

Intro. 1

hyperbolic Riemann surface

Intro. 1

hyperbolic volume

Intro. 1

Igusa curve

II. 3

indigenous bundle

Intro.2, I. 2

of restrictable type

I. 2

indigenous section

III. 2

induced $\mathcal{M} \mathscr{F}^{\nabla}$-object

V.1

infinitesimal Verschiebung

II. 2

intrinsic bundle

I. 2

Jacobson's formula

II. 2

Kähler metrics and Frobenius liftings

Intro. 2

Kodaira-Spencer morphism

I. 1, I.2

local compactification of a Frobenius lifting

IV . 4

local height of a Frobenius lifting

IV . 4

locally intrinsic bundle

I. 2

locally stable of dimension one

I. 1

log admissible covering

III. 2

$\log p$-divisible group

IV.2

marked

I. 1

$M \mathscr{F}^{\nabla}$-object

II. 2 
naive compactification of a Frobenius lifting

IV . 4

nilpotent indigenous bundle

II. 2

normalized $\mathbb{P}^{1}$-bundle with connection

I.1

ordinary curve, hyperbolically

II. 3

parabolically

II. 3

ordinary Frobenius lifting

III. 1

ordinary indigenous bundle

II. 3

ordinary locus

IV .1

$p$-curvature

II. 1

parabolic Riemann surface

Intro. 1

parabolic structure

I. 1

parabolic volume element

Intro. 1

partition curves

Intro. 1

pre-Schwarz structure

I. 1

pseudo-uniformizer

I. 1

quasiconformal equivalence class. $p$-adic

III. 3

quasiconformal function

Intro. 1

quasidisk

Intro. 1

real Riemann surface

Intro. 1

renormalized Frobenius pull-back

III. 2

ring of additive periods

III. 1

scheme of multiplicative periods

V.2

Schwarz structure

I. 1

Schwarzian derivative

I. 1

Serre-Tate parameter

IV .1

singular

I. 1

small affine

V.1

small parameters

V.1

smooth and unmarked

I. 1

stable curve

I. 2

stack of multiplicative periods

V.2

supersingular divisor

II. 2

tangential local system

III. 1 , III. 3

Teichmüller metric

Intro. 1

Teichmüller uniformization

Intro. 1

topological marking, $p$-adic

III. 3 
totally degenerate curve

I. 3

trianalytic constant

I. 1

function

I. 1

triformal function

I. 1

uniformizing Galois representation

III. 1

uniformizing $\mathcal{M F}^{\nabla}$-object

III. 1

Verschiebung of an indigenous bundle

II. 2

on $f_{*}\left(\omega_{X / S}^{\log }\right) \otimes^{2}(-D)$

II 2

morphism

II. 2

Weil-Petersson inner product

Intro. 1

Weil-Petersson metric

Intro. 1

Wolpert's coordinates of degeneration

Intro. 1

\section{Major Notation}

\begin{tabular}{|c|c|}
\hline $\mathscr{A}_{\Phi}$ & III. 1 \\
\hline $\mathscr{B}$ & Intro. 1 , II. 3 \\
\hline $\mathscr{B}_{\mu}$ & III. 3 \\
\hline$B^{+}(-)$ & V.1 \\
\hline $\mathscr{C}$ & I. 2, IV. 4 \\
\hline$\left(\mathscr{D}, \nabla_{\mathscr{D}}\right)$ & III. 2 \\
\hline$\Delta$ & V.1 \\
\hline$D_{\mathscr{E}}$ & II .2 \\
\hline$\left(\mathscr{D}_{E}, \mathscr{R}_{E}\right)$ & V.2 \\
\hline$d_{3}$ & I. 1 \\
\hline $\mathscr{D}_{S}: \mathscr{I}_{S}$ & V.1 \\
\hline $\mathscr{D}_{S}^{\text {Gal }}$ & V.1 \\
\hline$\left(\mathscr{D}_{y}, \mathscr{R}_{y}\right)$ & IV .3 \\
\hline $\mathscr{D}_{0}(a, b)$ & IV . 2 \\
\hline$\left(\mathscr{E} \mathscr{T}, \nabla_{\mathscr{E} \mathscr{E}}\right)$ & V.1 \\
\hline$E_{\&}$ & II. 2 \\
\hline$E_{X}^{\mathrm{Gal}}$ & V.1 \\
\hline$\left(\mathscr{E}_{\mathcal{N}}, \nabla_{\mathscr{E}_{1}}\right)$ & III. 2 \\
\hline$\eta_{\Phi}$ & III. 1 \\
\hline$E_{0}$ & V.1 \\
\hline $\mathbb{F}^{*}$ & II. 2, III .2 \\
\hline
\end{tabular}




\begin{tabular}{|c|c|}
\hline$G_{g, r} ; G_{\Delta}$ & II. 3 \\
\hline$G^{\log }$ & IV .2 \\
\hline $\mathrm{GL}^{ \pm}$ & I. 1 \\
\hline$G[\infty]$ & V.2 \\
\hline$H_{\mathscr{g}}$ & II. 2 \\
\hline$H_{Y}^{\mathrm{log}}$ & IV .3 \\
\hline $\mathrm{ht}(\Phi) ; \mathrm{ht}_{x}(\Phi)$ & IV .4 \\
\hline $\mathscr{H}_{x}$ & IV .3 \\
\hline$\ell(a b) 0$ & IV . 2 \\
\hline$g$ & I. 1 \\
\hline$\kappa$ & V.1 \\
\hline$\kappa_{A}$ & III. 1 \\
\hline$\kappa_{\beta}$ & III.1, III.3 \\
\hline$L_{\&} ; L_{\&}^{\times}$ & I.1 \\
\hline $\bar{M}_{g, r}$ & I. 2, III .2 \\
\hline $\bar{M}_{g, r}^{\text {ord }}$ & II. 3 \\
\hline$\mu=(\Delta, \varpi)$ & III. 3 \\
\hline$\overline{\mathcal{N}}_{g, r}$ & II . 2 \\
\hline$\overline{\mathcal{N}}_{g, r}^{\text {ord }}$ & II . 3, III. 2 \\
\hline$\Omega_{g, r}^{\mathrm{et}}$ & II .3 \\
\hline$\Omega_{\mathcal{N}}^{\mathrm{et}}$ & III . 3 \\
\hline$\Omega_{\Phi}^{\mathrm{et}}$ & III. 1 \\
\hline$\Omega_{S}^{\log }$ & $\mathrm{V} .1$ \\
\hline$\omega_{[}^{x}{ }^{\log , S^{108}}$ & I. 1 \\
\hline $\mathscr{O}_{\mathfrak{U}}^{\#}$ & I. 1 \\
\hline $\mathscr{P}$ & II. 1 \\
\hline$\left(\mathscr{P}, F^{\circ}(\mathscr{P}), \nabla_{\mathscr{P}}, \mathscr{P}_{\Phi}\right)$ & III. 1 \\
\hline$P_{\mathscr{B}}$ & II .2 \\
\hline $\mathscr{P}_{\text {et }}$ & III. 1 \\
\hline$\Phi_{g}$ & IV .1, V.1 \\
\hline$\Phi_{\mathcal{N}}^{\log }$ & III.2 \\
\hline$\Phi_{X}^{\log }$ & IV.1, V.2 \\
\hline$\Phi_{\mathscr{E}}^{\omega}$ & II .2 \\
\hline$\Phi_{\mathscr{E}}^{\tau}$ & II. 2 \\
\hline$\Pi_{1}, \Pi_{\infty}$ & V.1 \\
\hline$\varpi$ & III. 3 \\
\hline$P^{\log }$ & V.2 \\
\hline
\end{tabular}




\begin{tabular}{|c|c|}
\hline$\Psi$ & V.2 \\
\hline$\Psi_{\alpha \beta}$ & III. 1 \\
\hline$\Psi_{x}$ & IV .3 \\
\hline$Q$ & Intro. 1 \\
\hline$\overline{\mathscr{Q}}_{g, r}$ & II .2 \\
\hline$q_{\omega, \alpha}$ & III. 3 \\
\hline$q_{\omega, z}$ & II. 1 \\
\hline$q_{\theta}$ & IV . 1 \\
\hline$\rho$ & IV .1 \\
\hline$\rho_{\infty}$ & V.2 \\
\hline $8: \mathscr{S}^{x}$ & I. 1 \\
\hline$\overline{\mathscr{\theta}}_{g, r}$ & I. 3 \\
\hline$\overline{\mathscr{S}}_{g, r}^{\mathrm{adm}}$ & II .2 \\
\hline $\mathscr{T}$ & II. 1 , IV.1 \\
\hline $\mathscr{T}^{1 / 2}$ & IV .1 \\
\hline$\Theta_{g}$ & II .2 \\
\hline$\Theta_{g, r}^{\mathrm{et}}$ & II .3 \\
\hline$\Theta_{N}^{\mathrm{e}} \mathrm{t}$ & III. 3 \\
\hline$\Theta_{\Phi}^{\text {et }}$ & III. 1 \\
\hline$\Theta_{\mathrm{F}^{*}}$ & III.2 \\
\hline$\Theta_{\mathscr{E}}^{*}$ & II .2 \\
\hline $\mathcal{U}^{\mathrm{b} 1}$ & I. 1 \\
\hline${\widehat{\mathcal{U}^{b}}}^{b_{1}}$ & I. 1 \\
\hline $\mathcal{U}^{\text {can }}$ & III.1, III. 3 \\
\hline $\mathcal{U}^{\mathrm{tr}}$ & I. 1 \\
\hline$\widehat{\mathscr{U}}^{\mathrm{tr}}$ & I. 1 \\
\hline$V_{B}$ & II. 2 \\
\hline $\bar{V}_{g, r}$ & II . 2 \\
\hline $\mathscr{V}_{\mu, \beta}$ & III. 3 \\
\hline$X(a, b)^{\text {ord }}$ & IV .3 \\
\hline$X^{\text {ord }}:\left(X^{\log }\right)^{\text {ord }}$ & IV .1 \\
\hline$X_{0}(n)$ & IV .2 \\
\hline
\end{tabular}


